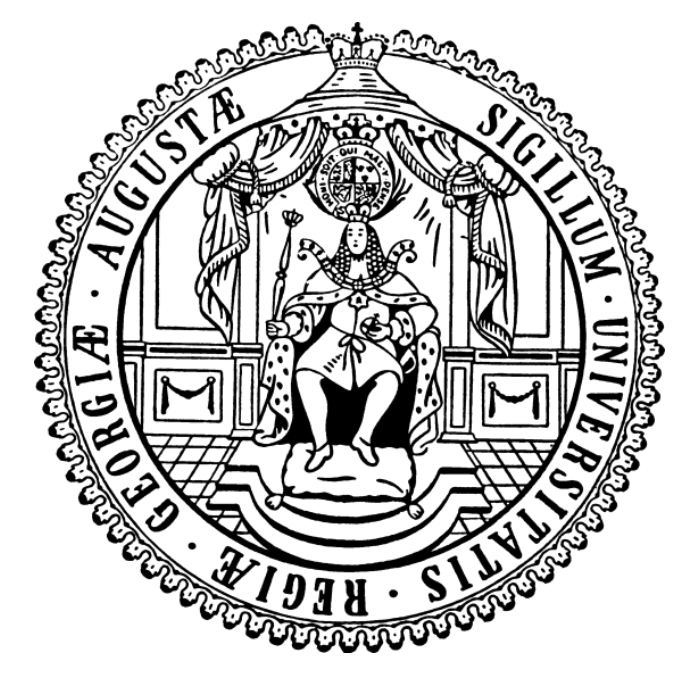

\title{
Advanced NMR Methodology for the Investigation of Organometallic Compounds in Solution
}

\author{
Dissertation \\ zur Erlangung des mathematisch-naturwissenschaftlichen Doktorgrades \\ „Doctor rerum naturalium“ \\ der Georg-August-Universität Göttingen \\ im Promotionsprogramm CaSuS \\ der Georg-August University School of Science (GAUSS) \\ vorgelegt von \\ Ann-Christin Pöppler \\ aus Hofgeismar
}

Göttingen, 2013 


\section{Betreuungsausschuss}

Prof. Dr. Dietmar Stalke, Institut für Anorganische Chemie

Prof. Dr. Philipp Vana, Institut für Physikalische Chemie

Dr. Michael John, Institut für Anorganische Chemie

\section{Mitglieder der Prüfungskommission}

Referent: $\quad$ Prof. Dr. Dietmar Stalke

Korreferent: Prof. Dr. Philipp Vana

weitere Mitglieder

Dr. Michael John, Institut für Anorganische Chemie

Prof. Dr. Ulf Diederichsen, Institut für Organische und Biomolekulare Chemie

Dr. Inke Siewert, Institut für Anorganische Chemie

Jun.-Prof. Dr. Ricardo A. Mata, Institut für Physikalische Chemie

Tag der mündlichen Prüfung: 24.06.2013 
für

Mama, Papa und Jana!

Die besten Entdeckungsreisen macht man, indem man die Welt mit anderen Augen betrachtet. 



\section{Table of Contents}

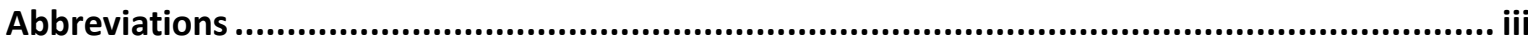

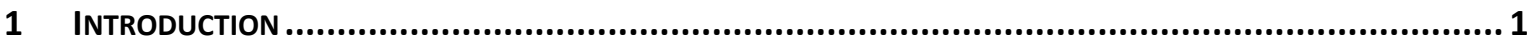

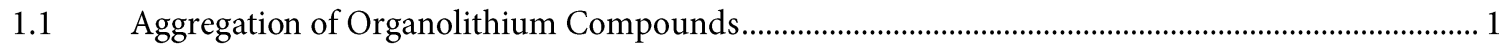

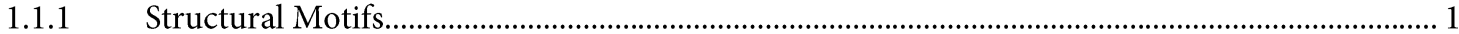

1.1.2 Structure Elucidation by X-ray Diffraction Experiments............................................................. 3

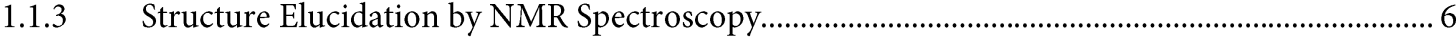

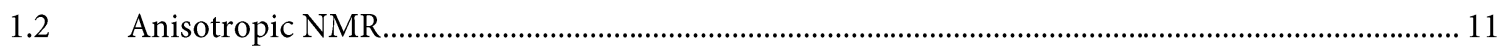

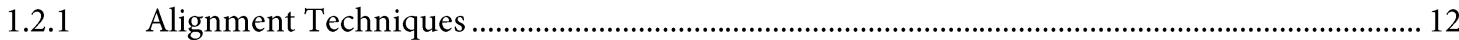

1.2.2 Residual Anisotropic NMR Parameters (RDCs, RQCs and RCSAs) .......................................2 20

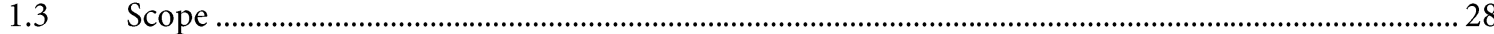

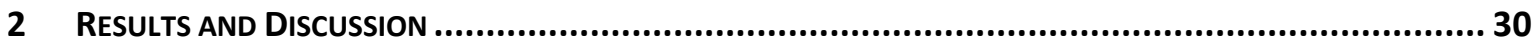

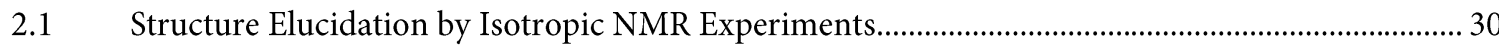

2.1.1 Mixed Crystalline Lithiumorganics and Interconversion in Solution..........................................30

2.1.2 Aggregation of Donor Base Stabilized 2-Thienyllithium in Solution.......................................... 36

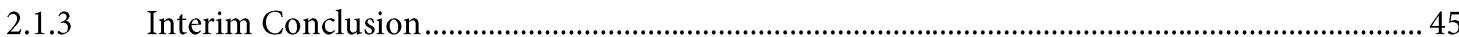

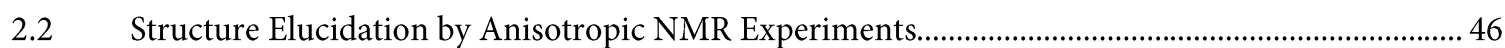

2.2.1 Employment of Polystyrene Gels for the Analysis of Organolithium Compounds ................... 46

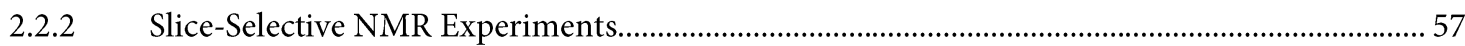

2.2.3 Investigations of Different Polymer Systems: PBA and Modification of PS.............................. 69

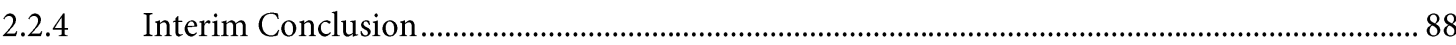

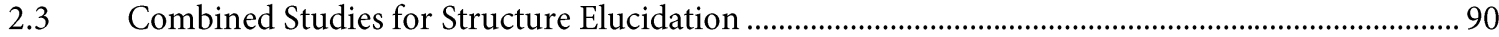

2.3.1 Study of $i \mathrm{PrMgCl}$ and its Turbo Analogue in THF ...................................................................90

2.3.2 Investigation of ThiLi-Diglyme by NMR Spectroscopy and DFT-Calculations.........................99

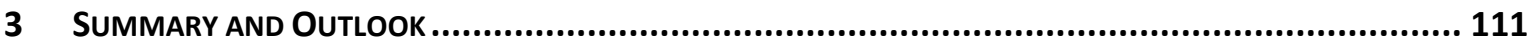

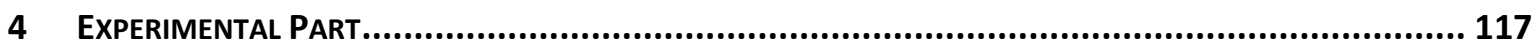

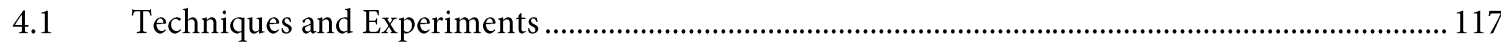

4.1.1 Handling of Air- and Moisture-Sensitive Compounds ............................................................ 117

4.1.2 NMR Techniques and Experiments ........................................................................................... 117

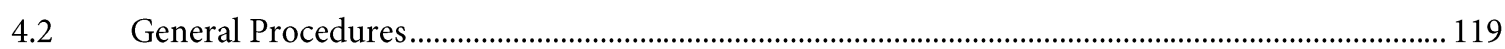

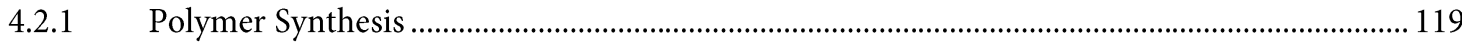

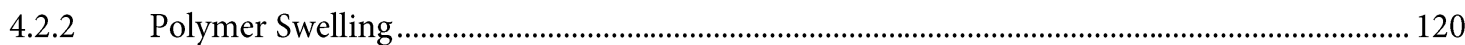

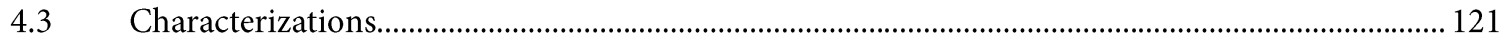

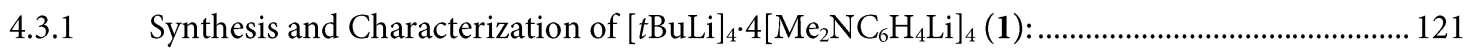

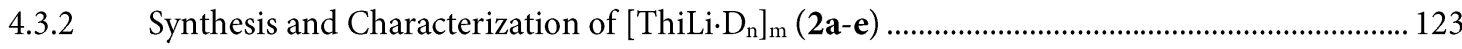

4.3.3 Characterization of (-)-Menthol (3) ..................................................................................... 126

4.3.4 Characterization of Lithium hexamethyldisilazane (4) ......................................................... 127

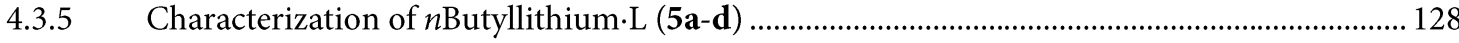

4.3.6 Characterization of Lithiumtetrafluoroborate $(\mathbf{6})$................................................................... 128 


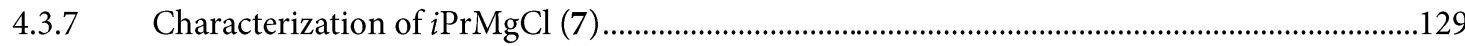

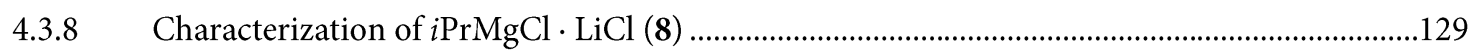

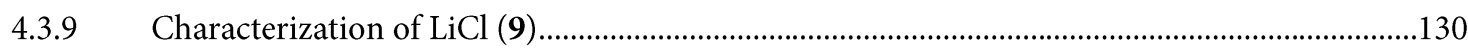

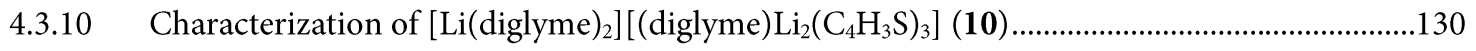

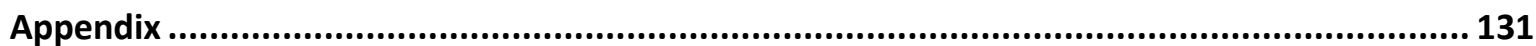

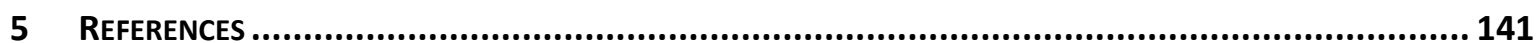




\section{Abbreviations}

\begin{tabular}{|c|c|}
\hline AIBN & 2,2'-Azobis(2-methylpropionitrile) \\
\hline ATP & adenosine triphosphate \\
\hline ATRP & atom transfer radical polymerization \\
\hline BA & butyl acrylate \\
\hline BDDA & 1,4-butanediol diacrylate \\
\hline $\mathrm{Bn}$ & benzyle \\
\hline $\mathrm{CDB}$ & cyanopropyldithiobenzoate \\
\hline$c \mathrm{Hex}$ & cyclo-hexyl \\
\hline COSY & correlation spectroscopy \\
\hline CPMG & Carr-Purcell-Meiboom-Gill \\
\hline CTA & chain transfer agent \\
\hline DBP & dibenzoyl peroxide \\
\hline DHCP & dihexanoylphosphatidylcholine \\
\hline DMA & $N, N$-dimethylaniline \\
\hline DME & dimethoxyethane \\
\hline DMF & dimethylformamid \\
\hline DMPC & dimyristoylphosphatidylcholine \\
\hline DMSO & dimethylsulfoxide \\
\hline DOSY & ciffusion ordered spectroscopy \\
\hline DVB & divinyl benzene \\
\hline EA & ethyl acrylate \\
\hline EFG & electric field gradient \\
\hline ЕРНТ & s-ethyl-propane-2-ylonate-s'-hexyl-trithiocarbonate \\
\hline EPR & electron paramagnetic resonance \\
\hline ESI & electrospray ionization \\
\hline Et & ethyl \\
\hline $\mathrm{Et}_{2} \mathrm{O}$ & diethyl ether \\
\hline EXSY & exchange spectroscopy \\
\hline HOESY & heteronuclear overhauser enhancement spectroscopy \\
\hline HMBC & heteronuclear multiple bond correlation \\
\hline HSQC & heteronuclear single quantum correlation \\
\hline ID & inner diameter \\
\hline$i \operatorname{Pr}$ & iso-propyle \\
\hline LC & liquid crystal \\
\hline
\end{tabular}


LiHMDS

MAS

$\mathrm{Me}$

MRI

$n \mathrm{BuLi}$

NMP

NMR

NOE

NOESY

PAA also $\mathrm{PH}$

PAN

PBLG

PCBLL

PDMS

PELG

$\mathrm{Ph}$

PMDETA

PMMA

PS

PVAc

RAFT

RCSA

RDC

$\mathrm{RF}$

RQC

RINMR

SAG

ssDNA

SSE

SSIP

tBuLi

THF

TMEDA

VA

VASS lithium hexamethyldisilazane

magic angle spinning

methyl

magnetic resonance imaging

n-butyllithium

nitroxide-mediated polymerization

nuclear magnetic resonance

nuclear overhauser effect

nuclear overhauser enhancement spectroscopy

polyacrylamide

poly(acrylonitrile)

poly- $\gamma$-benzyl-1-glutamate

poly-e-carbobenzyloxy-l-lysine

poly(dimethylsiloxane)

poly- $\gamma$-ethyl-1-glutamate

phenyl

$N, N, N^{\prime}, N^{\prime \prime}, N^{\prime \prime}$-pentamethyldiethylenetriamine

poly(methyl methacrylate)

polystyrene

polyvinylacetate

reversible addition fragmentation chain transfer

residual chemical shift anisotropy

residual dipolar coupling

radiofrequency

residual quadrupolar coupling

rapid injection NMR

strain induced alignment in a gel

single stranded deoxyribonucleic acid

slice-selective excitation

solvent-separated ion pair

tert-butyllithium

tetrahydrofurane

$N, N, N$ ', $N$ '-tetramethylethylene-1,2-diamine

variable angle experiment

variable angle sample spinning 


\section{INTRODUCTION}

\subsection{Aggregation of Organolithium Compounds}

\subsubsection{Structural Motifs}

Organolithiums ${ }^{[1]}$ range amongst the most versatile reagents in many fields of chemistry. Their broad scope of substrates and manifold synthetic applications have been underlined by various reviews and book chapters. ${ }^{[2]}$ Apart from general synthetic approaches, organolithiums have gained more and more importance in the field of selective reaction sequences. Among those are regio-, diastereo-, and enantioselective reactions that enable to build up more complex molecular structures. ${ }^{[3]}$ They have also caught the attention of industrial chemists in various areas of asymmetric processes (Scheme 1.1) and polymer syntheses for which efficient routes involving organolithium species have been developed. ${ }^{[4]}$

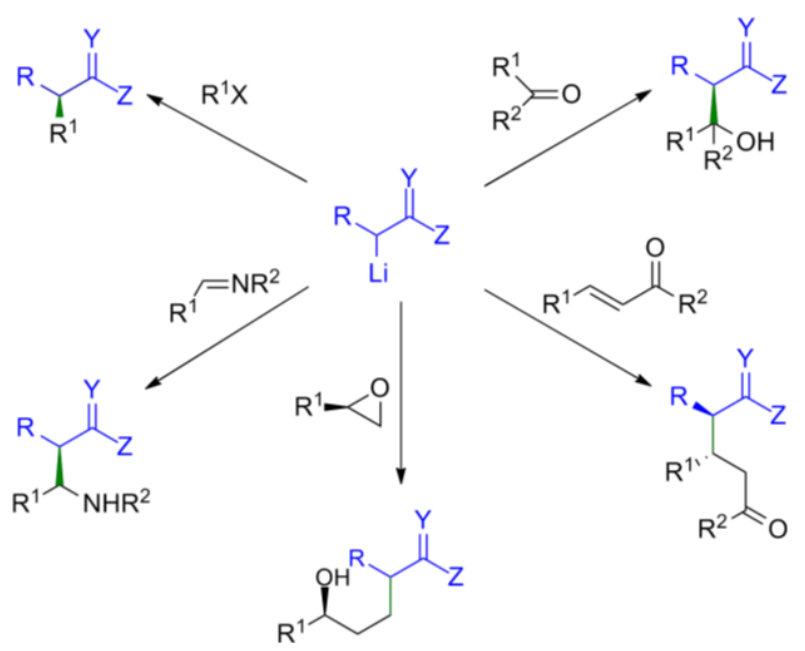

Scheme 1.1: Various reaction pathways for organolithiums in asymmetric organic synthesis. $\mathrm{X}=$ leaving group; $\mathrm{Y}=\mathrm{O}, \mathrm{NR}$, or $\mathrm{CR}_{2} ; \mathrm{Z}=\mathrm{H}, \mathrm{OR}, \mathrm{NR}_{2} \cdot{ }^{[4]}$

In many reaction schemes, the organolithium reactant is depicted as RLi, in other words as a monomer. However, it has been known since the 1960s when the first crystal structure of ethyllithium was published ${ }^{[5]}$ that organolithiums form oligomers and highly aggregated species. Since then, a variety of structural principles could be identified which helps to understand the nature of organolithium compounds. From this knowledge conclusions can be drawn about structure-reactivity relationships that enable synthetic chemist to adequately tune these potent reagents for their purposes. 

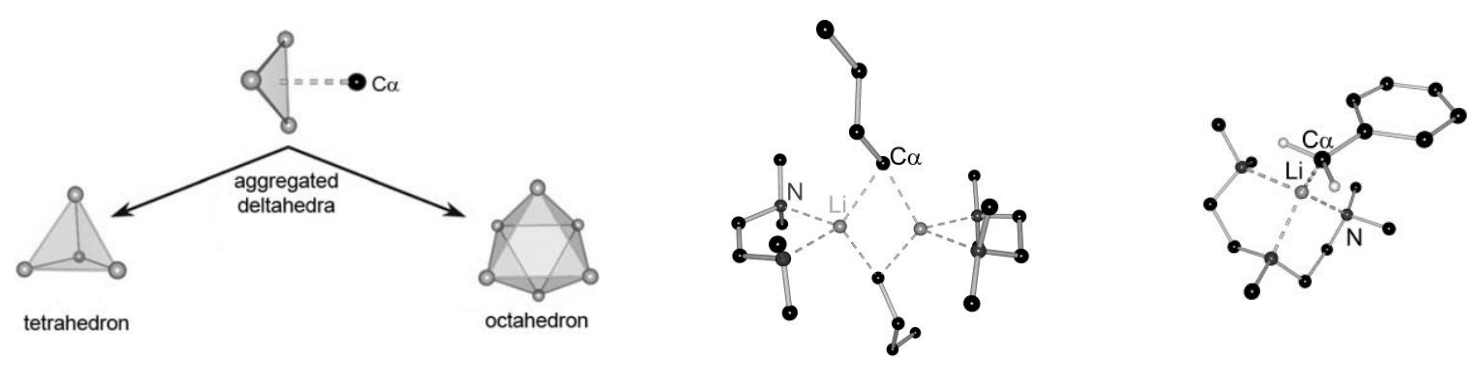

Figure 1.1: Very important building blocks in organolithium chemistry consisting of tetrameric, hexameric, dimeric and monomeric units (from left to right) ${ }^{[6]}$

Common oligomers of neutral organolithium compounds $\mathrm{R}_{\mathrm{m}} \mathrm{Li}_{\mathrm{n}}$ with $\mathrm{m}=\mathrm{n}$ are hexameric, tetrameric and dimeric structures. Because the formation of such oligomers does not only depend on electrostatics but also on the steric demand of the organic residue and the lithium coordination sphere, higher aggregates can be deliberately disaggregated to smaller structural motifs. The addition of Lewis bases, for instance, favours disaggregated adducts and even monomeric arrangements with a single lithium carbon bond might thereby be achieved (Figure 1.1). The key features of these aggregates have been thoroughly described in literature and therefore will be only shortly discussed in this context. ${ }^{[6-7]}$

A significant building principle in these structures is the arrangement of the lithium atoms to $\mathrm{Li}_{3}$ triangles which connect to form higher aggregates like octahedrons and tetrahedrons. These triangles are $\mu_{3}$-capped by the $C_{a}$-atoms of the organic residue. Whilst for the tetrahedral structure all surfaces are $\mu_{3}$-capped, the octahedral arrangement displays of two uncapped $\mathrm{Li}_{3}$ triangles on opposite sides. Thereby, all lithium atoms reach their preferred coordination number of four. In contrast, the key feature in dimers is a $\mathrm{Li}_{2} \mathrm{C}_{2}$ fourmembered ring. The coordination sphere herein is generally filled by the donor atoms of an applied Lewis base. Reducing the number of lithium carbon contacts further leads to the formation of monomeric structures. Again donating Lewis bases stabilize this structural principle except that an increased bulkiness of the ligands is also required. Less represented but similar aggregates in the form of trimers as well as octamers and nonamers have also been ascertained for organolithiums. ${ }^{[8]}$

Moving away from neutral $\mathrm{R}_{\mathrm{m}} \mathrm{Li}_{\mathrm{n}}$ aggregates with $\mathrm{m}=\mathrm{n}$ one arrives at charged species that contain either an excess of $\mathrm{R}$ or $\mathrm{Li}(\mathrm{m} \neq \mathrm{n})$. With a solvent-separated ion pair (SSIP) being the simplest representative, lithium lithiates have long been known as so called triple ions. These "ate" type complexes are composed of an anion consisting of two negatively charged organic residues and one lithium cation along with a solvent-coordinated cation building an overall solvent-separated ion pair. ${ }^{[9]}$ Very recently, the new form of a pentuple ion, named after the five ions building the negatively charged building block to which a diglyme coordinated lithium cation serves as the counterion, has been established in our 
workgroup. ${ }^{[10]}$ This building block has not been characterized in organollithium chemistry before, but it is not as unusual in other fields and has been theoretically supported for lithium chloride in THF (Figure 1.2). ${ }^{[1]}$ Additionally, it has to be considered that in many cases no specific aggregate but multiple aggregates may simultaneously exist in solution.
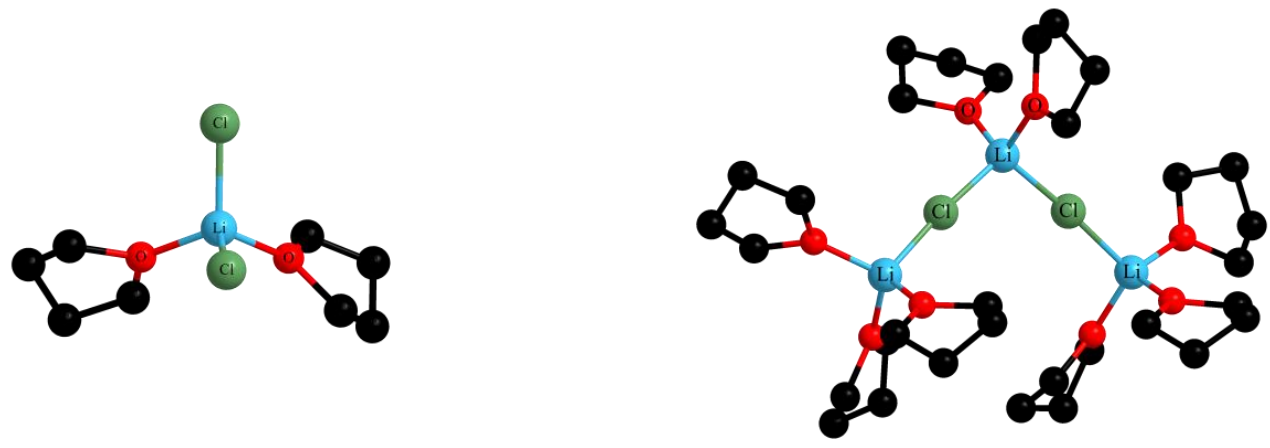

Figure 1.2: Calculated structures for a solution of lithium chloride in THF: A triple ion (left) with the lithium atom as centre of the complex anion and a quintuple ion (right) with three lithium atoms and two chloride atoms. In all cases, the coordination number of the lithium atoms is raised to four by additional coordination of THF molecules. ${ }^{[11]}$

To gain information about aggregation and important structural features of organolithiums that may be used to deduce structure-reactivity relationships a variety of analytical methods is available. Among those, X-ray diffraction and MAS NMR experiments are the key methods for the analysis of the solid state properties, whereas cryoscopic molecular mass measurements ${ }^{[12]}$ and high-resolution NMR provide insight into the liquid state, respectively. Calculations like $a b$ initio prediction of the stability or theoretical charge density studies of various aggregates have also been thoroughly investigated. The following chapters will be primarily concerned with the structure elucidation of organolithiums by X-ray diffraction experiments and high-resolution NMR studies.

\subsubsection{Structure Elucidation by X-ray Diffraction Experiments}

Stucky et al. stated in 1970 that "the lack of structural knowledge of organolithium compounds is in sharp contrast to their widespread use" when introducing their crystal structure analysis of benzyllithium triethylenediamine. ${ }^{[13]}$ At that time, only three structures of organolithium compounds had been determined, namely, the first crystal structure of tetrameric ethyllithium by Dietrich, ${ }^{[5]}$ the investigation of tetrameric methyllithium via powder diffraction by Weiss et al. ${ }^{[14]}$ and the analysis of $\operatorname{LiAl}\left(\mathrm{C}_{2} \mathrm{H}_{5}\right)_{4}$ by Brown and co-workers. ${ }^{[15]}$ However, crucial progress was not made until more sophisticated methods for crystal structure analyses of air- and moisture-sensitive compounds above all cryo techniques had been developed. ${ }^{[16]}$ While the above mentioned 
crystal structures were measured at room temperature, ${ }^{[17]}$ Veith and Bärnighausen reported a crystal structure for which data collection took place at $-130{ }^{\circ} \mathrm{C}$ and introduced an apparatus on which also the crystal selection and transfer was afforded at low temperatures. ${ }^{[18]}$ In the following, a variety of different devices for crystal handling (crystallization vessel, mounting apparatus, etc.) were developed. ${ }^{[19]}$ Subsequently, the procedure of crystal mounting was further improved by a combination of a protecting oil droplet, in which the crystal is shielded against the atmospheric air, and a cooling device. An apparatus that allows constant low temperature handling of crystals was promoted by Stalke et al. in 1993 and is schematically portrayed in Figure 1.3. ${ }^{[20]}$ It significantly simplifies the handling of very air- and moisture-sensitive compounds.

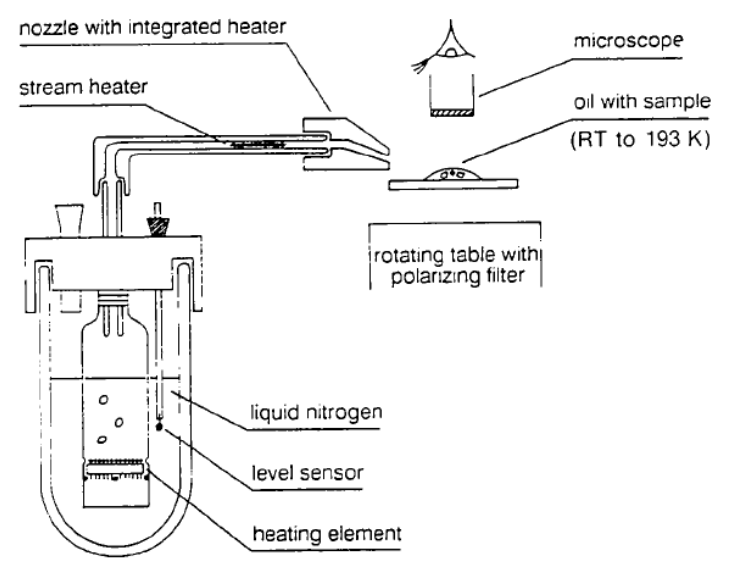

Figure 1.3: Schematic overview of the low-temperature device introduced by Kottke and Stalke in 1993.[20]

The newest piece of equipment added to the above mentioned cooling technique is a copper block cooled with dry ice that allows the safe mounting of even more sensitive and reactive crystals. ${ }^{[21]}$ Meanwhile, many important crystal structures of highly reactive organolithium compounds have been analysed. ${ }^{[7 a, 22]}$ Of the prominent alkyllithium compounds several hexameric structures, e.g. $[n \mathrm{BuLi}]_{6},{ }^{[23]}[i \mathrm{PrLi}]_{6}{ }^{[24]}$ and $[c \mathrm{HexLi}]_{6}{ }^{[25]}$ could be crystallized from hydrocarbon solution and subsequently analysed by X-ray diffraction. In contrast to these hexameric structures, the tetrameric arrangement could be confirmed for $[\mathrm{MeLi}]_{4}^{[26]}$ and $[\mathrm{EtLi}]_{4}^{[27]}$ whereas $[t \mathrm{BuLi}]_{4}$ was newly added to this series of Lewis-basefree structures. ${ }^{[23]} \mathrm{A}$ decrease of the degree of aggregation is observed when neutral Lewis bases are added which is an obvious area of interest as those crystallized solvent adducts supposedly mirror the polar reaction mixture utilized in synthesis. The verification of this assumption by a corresponding analysis in solution is therefore important and also a major aim of this work. Due to the huge variety of base / organolithium compositions, only few examples will be discussed herein. 
Apart from the overall structure of the organolithium reagents, the nature of the lithium carbon bond itself has been a subject of constant discussion and will be further explained in the following. Whilst the oxygen-donor THF is able to disaggregate $[n \mathrm{BuLi}]_{6}$ to form tetrameric units of the type $[(\mathrm{THF}) n \mathrm{BuLi}]_{4}$, TMEDA yields dimers [(TMEDA) $\left.n \mathrm{BuLi}\right]_{2}$ (besides $\left.\left[(\mathrm{TMEDA})_{2}(n \mathrm{BuLi})_{4}\right]_{\infty}\right)$ in the solid state (Figure 1.4). ${ }^{[28]}$
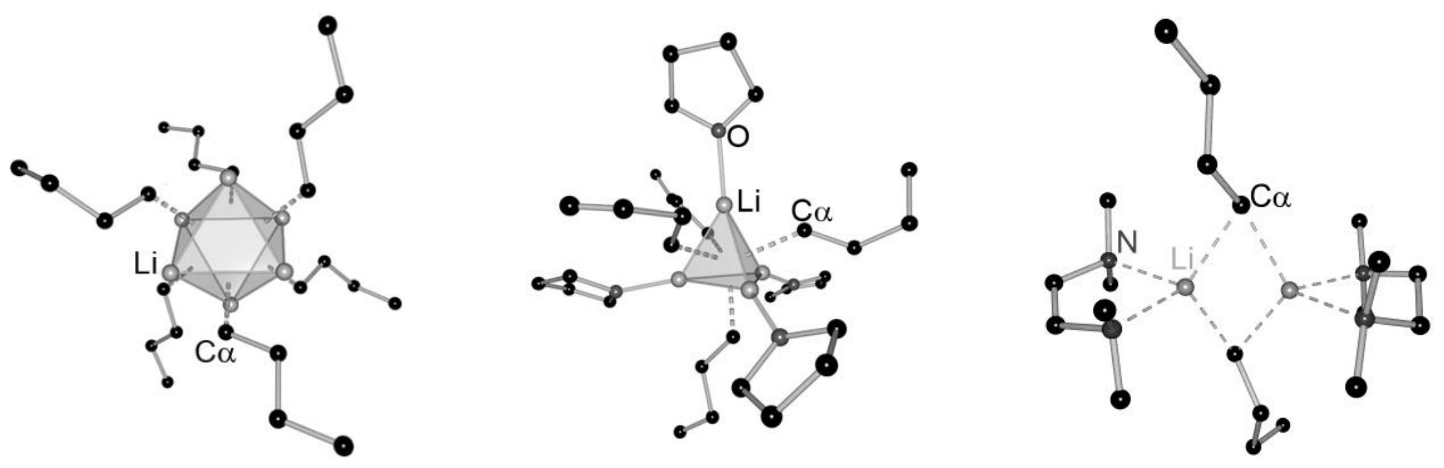

Figure 1.4: Solid state structures of $[n \mathrm{BuLi}]_{6},[(\mathrm{THF}) n \mathrm{BuLi}]_{4}$ and $[(\mathrm{TMEDA}) n \mathrm{BuLi}]_{2}$ (from left to right).

In this series of deaggregation the $\mathrm{Li}^{-} \mathrm{C}_{\alpha}$ distance decreases with decreasing aggregation number due to fewer lithium carbon contacts and thus a smaller charge distribution. The smallest $\mathrm{Li}-\mathrm{C}_{\alpha}$ distance has been observed for monomeric species displaying only one lithium-carbon bond. Very bulky ligands like (-)-sparteine are able to stabilize monomeric $\left[\{(-)\right.$-sparteine $\}$ tBuLi] units. ${ }^{[29]}$ Due to the chiral nature of this Lewis base it is a valuable additive for the formation of asymmetric compounds via enantioselective formation of new carbon-carbon bonds.

Apart from the bond length, the polarity of the lithium-carbon bond is an important attribute of organolithiums. Supported by theoretical and experimental approaches, it has been controversially discussed to what extent this bond is more of ionic or covalent character. ${ }^{[31]}$ For this purpose the electron density analysis provided an answer revealing that the carbanionic character of $\mathrm{C}_{\alpha}$ increases with the polarity of the bond. Advances in theoretical and experimental electron density studies and the investigation of various molecular systems finally led to the recognition of the bond to be mainly,

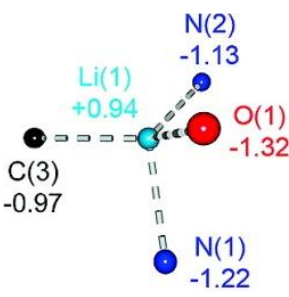

Figure 1.5: Integrated atomic charges of $\operatorname{Li}(1)$ and its coordination sphere [e] in $(R, S)$-2.quinuclidine. ${ }^{[30]}$ that is to say $80-90 \%$, ionic (Figure 1.5). ${ }^{[30,32]}$

It is thus not necessary to further underline the vital and fundamental role X-ray diffraction plays in the understanding of organolithiums and the fascinating variety of structures present in the solid state. 


\subsubsection{Structure Elucidation by NMR Spectroscopy}

As reactions with organolithiums are generally carried out in solution, the analysis of structural features in a solvent environment is of utmost importance. To do so, highresolution NMR spectroscopy has become indispensable. During the last decades, many different approaches to analyse the structure, aggregation and dynamics of organolithiums in both polar and non-polar solvents have been made and also constantly reviewed ever since ${ }^{[33]}$ When the structure elucidation of these important synthons was still in its infancy, chemical shifts $\left({ }^{1} \mathrm{H},{ }^{13} \mathrm{C},{ }^{6} \mathrm{Li}\right.$ and $\left.{ }^{7} \mathrm{Li}\right)$ were consulted to gain knowledge about electronegativity and aggregate symmetry. ${ }^{[34]}$ Thereby, it became quite obvious, that the employment of ${ }^{6} \mathrm{Li}$ or ${ }^{7} \mathrm{Li}$ chemical shifts is rather unreliable due to a small chemical shift range and a severe dependence on substituents, concentration and the applied solvent. ${ }^{[35]}$ These factors also influence the aggregation being another drawback of structure elucidation on the basis of lithium chemical shifts. However, ${ }^{13} \mathrm{C}$ chemical shifts turned out to be a significant benchmark system as was presented by Thomas et al. for various organolithiums both with straight or branched alkyl chains. ${ }^{[3]}$ His measurements were performed in the non-polar solvent cyclopentane allowing him to distinguish, for example, between the hexameric, octameric and nonameric aggregates for ethyllithium as well as propyllithium at low temperatures. In the following, the focus moved slightly away from the chemical shift itself to the powerful lithium-carbon coupling that can be extracted at low temperatures and the resulting multiplicity of the signal. The first ${ }^{13} \mathrm{C}^{-} \mathrm{Li}$ spin-spin coupling was observed for methyllithium by researchers of The Dow Chemical Company in 1968 and can be seen as a cornerstone for the comprehension of the behaviour of organolithiums in solution. ${ }^{[37]}$ They used ${ }^{13} \mathrm{C}$-enriched methyllithium and lithium iodide as reference in THF solution and later on expanded their studies to ether and triethylamine as solvent systems. Investigations in this area were governed by isotope labelling $\left({ }^{6} \mathrm{Li},{ }^{13} \mathrm{C}\right)$, and experimental evidence led to the development of the following rule of thumb for the magnitude of the coupling constant which has been optimized and theoretically fortified only recently: ${ }^{[38]}$

$$
{ }^{{ }^{13} \mathrm{C}-{ }^{6} \mathrm{Li}}=\frac{17 \pm 2 \mathrm{~Hz}}{n}
$$

with $n$ being the number of equivalently bonded ${ }^{6} \mathrm{Li}$ nuclei. For this matter it is vital to differentiate between dynamic and static aggregates, because in the case of a fluxional behaviour of the aggregates, $n$ represents the aggregation state. In contrast, lowering the temperature freezes the intra-aggregate dynamics and leads to a decrease in the number of coupling partners, e.g. from four to three in case of a tetrameric unit. Appendant to the 
magnitude of the coupling constant, the multiplicity of the ${ }^{13} \mathrm{C}$ signal likewise affords the identification of the aggregation, because it is caused by the closest neighbours at low temperatures, and by all incorporated lithium atoms if the measurement temperature is not low enough to sufficiently eliminate intra-aggregate exchange. Thus, the general rule $2 I n+1$ (with $I=1$ for ${ }^{6} \mathrm{Li}$ and $I=3 / 2$ for ${ }^{7} \mathrm{Li}$, respectively) can be employed to deduce the required multiplicity (Table 1.1). In cases of very high multiplicity, the differentiation of the outermost signals with very small intensities might be hampered by signal-to-noise issues. Therefore, a lineshape analysis, i.e. a comparison of simulations for the $\mathrm{C}_{\alpha}$-multiplet of different aggregation states with experimental data, often provides a more definite answer. Many surveys regarding the structure of organolithium in solution have been carried out on the basis of these parameters. ${ }^{[\mathrm{b}, 39]}$

Table 1.1: ${ }^{13} \mathrm{C}$ NMR multiplets due to ${ }^{6} \mathrm{Li}_{n}{ }^{13} \mathrm{C}$ and ${ }^{7} \mathrm{Li}_{n}{ }^{13} \mathrm{C}$ coupling. Multiplets show equal coupling within each $\mathrm{Li}_{n}{ }^{13} \mathrm{C}$ site. ${ }^{[33 \mathrm{~d}]}$

\begin{tabular}{cccc}
\hline$n$ & Aggregation State & ${ }^{6}$ Li Multiplet & ${ }^{7}$ Li Multiplet \\
\hline 1 & monomer & $1: 1: 1$ & $1: 1: 1: 1$ \\
2 & dimer & $1: 2: 3: 2: 1$ & $1: 2: 3: 4: 3: 2: 1$ \\
3 & static tetramer & $1: 3: 6: 7: 6: 3: 1$ & $1: 3: 6: 10: 12: 12: 10: 6: 3: 1$ \\
\hline
\end{tabular}

Phenyllithium ( $\mathrm{PhLi})$ and $n$-butyllithium $(n \mathrm{BuLi})$ are exemplarily discussed herein as prominent examples of long-term investigated objects in organolithium chemistry. The first NMR spectroscopic study of phenyllithium was carried out by Fraenkel et al. in 1969. ${ }^{[40]}$ Therein, only chemical shifts were discussed and further analytical data was provided later on by Seebach and Jackman during the $1980 \mathrm{~s},{ }^{[396,41]}$ which was also when the solid state structure of phenyllithium in ether and with additives like the Lewis bases TMEDA $=N, N, N$, $N$ '-tetramethylethylene-1,2-diamine and PMDETA $=N, N, N, N^{\prime}, N^{\prime \prime}$ pentamethyldiethylenetriamine was established (Scheme 1.2). ${ }^{[42]}$
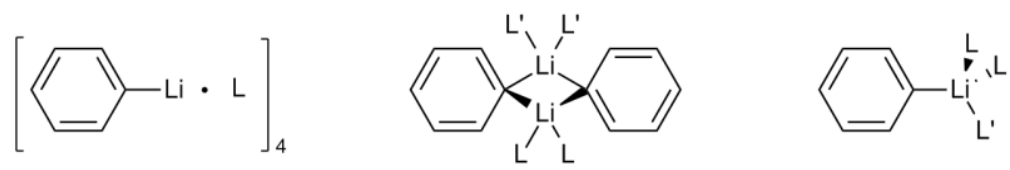

Scheme 1.2: Different aggregation states observed for phenyllithium in the solid state. Addition of Lewis bases $L$ (e.g. TMEDA or PMDETA) yields the disaggregated arrangements.

The moderate reactivity of $\mathrm{PhLi}$ fortunately allows in-depth analyses of organolithium / Lewis base mixtures due to the fact that no excessive destruction by reaction with the solvent or additive is observed. Finally, Reich et al. could significantly complement the ongoing structure elucidation of phenyllithium by reporting fully resolved ipso carbon 
signals for all PhLi aggregates, a rich number of complexes with polar additives and their corresponding reactivity. ${ }^{[43]}$ They clarified $\mathrm{PhLi}$ to form a tetramer/dimer mixture in ethereal solution and a dimer/monomer mixture in THF. By addition of Lewis bases, e.g. THF or TMEDA, these equilibria can be shifted to smaller aggregates reaching a minimum by coordination of PMDETA. Irrespective of the solvent environment, only monomeric units are obtained upon addition of PMDETA which are considerably more reactive than the TMEDA-coordinated adducts.

One of the most commonly used alkyllithium compounds, $n \mathrm{BuLi}$, is more difficult to study. Above all, no ${ }^{13} \mathrm{C}-{ }^{6} \mathrm{Li}$ coupling can be observed for linear alkyllithiums which is presumably due to dynamic processes of the flexible organic residues. McGarrity and Ogle could reveal that $n \mathrm{BuLi}$ in THF exists as a tetramer-dimer equilibrium. ${ }^{[4]}$ The corresponding rates could be obtained from ${ }^{7} \mathrm{Li} \mathrm{NMR}$ experiments and the presence and influence of alkoxides in the scrutinized solutions accentuated. Subsequent employment of the Rapid Injection NMR (RINMR) method, which they contrived earlier in the 1980s, facilitated an evaluation of their reactivity. ${ }^{[45]}$ Thereby, the dimer was judged to be more reactive than the tetramer and replacement of alkyl groups by alkoxy moieties resulted in a further increase in reactivity.

In the following years, the focus shifted towards the application of $2 \mathrm{D}$ shift correlations to gain structural information in solution. Here, a differentiation has to be done with respect to the nature of the observed correlation to be either scalar (through bonds) or dipolar (through space). The latter correlation is based on the (hetero) Nuclear Overhauser Effect (NOE) and a correlation is therefore observed in the spectrum if two nuclei are in close spatial proximity so that they contribute to the dipolar relaxation of the other. The first homonuclear 2D experiments to observe NOE phenomena were developed by Ernst and co-workers in the beginnings of the $1980 \mathrm{~s}^{[46]}$ and further expanded to the observation of two-dimensional Heteronuclear NOE (HOESY) experiments by Levy et al. ${ }^{[4]}$ and Rinaldi. ${ }^{[48]}$ In the subsequent work, $Y u$ and Levy were able to successfully implement this experiment in the analysis of camphor, fluoranthene and adenosine triphosphate (ATP). ${ }^{[49]}$ The benefit for organolithium chemistry was finally put forward by Bauer and $v . R$. Schleyer who were the first to apply the beforehand mentioned experiment to the detection of short ${ }^{6} \mathrm{Li},{ }^{1} \mathrm{H}$ distances at natural ${ }^{6} \mathrm{Li}$ abundance. ${ }^{[50]}$ This work was focused on a possible application for spectral assignment of lithiated organic molecules as well as for the prediction of the reactive position in case of a second lithiation step. An exemplary HOESY spectrum of $\mathrm{Me}_{2} \mathrm{CuLi} \cdot \mathrm{LiCN}$ in THF is displayed in Figure 1.6. Crosspeaks to the THF molecules indicate coordination of the lithium atom and a crosspeak to the methyl group underlines the close vicinity of lithium and the corresponding $\mathrm{Me}_{2} \mathrm{Cu}$-fragment. As 
crosspeak intensities depend on the inverse $6^{\text {th }}$ power of the distance, the method also represents a powerful source of distance restraints. This only hints at the enormous significance of HOESY in the general field of organometallic and in particular organolithium chemistry. ${ }^{[5}$

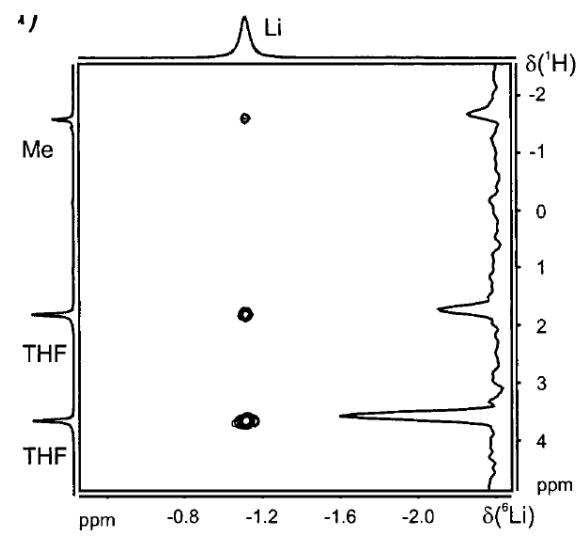

Figure 1.6: Exemplary ${ }^{1} \mathrm{H},{ }^{6} \mathrm{Li}-\mathrm{HOESY}$ spectrum of $\mathrm{Me}_{2} \mathrm{CuLi} \cdot \mathrm{LiCN}$ in $\mathrm{THF}$ at $213 \mathrm{~K}$ recorded with a mixing time of 1.7 s. $^{[52]}$

A similarly intriguing progress was recently made in the field of diffusion-ordered NMR techniques that allow the study of self-diffusion arising from random translational (Brownian) motion. The translational motion of a molecule is faster the smaller its hydrodynamic radius which is embodied by the Stokes-Einstein equation (1-2) for an ideal spherical molecule. ${ }^{[53]}$

$$
D=\frac{k_{B} T}{6 \pi \eta r_{S}}
$$

This equation expresses the diffusion coefficient $D$ as a function of the temperature $T$ and viscosity $\eta$, both being properties of the solvent environment, and inverse proportional to the radius of the spherical molecule.

The important advantages of this spectroscopic method are its fully non-invasiveness and the possibility to separate the individual components of complex component mixtures while simultaneously facilitating the characterization of these compounds with respect to size and other structural features. The (pseudo) two-dimensional DOSY experiment was promoted by Morris and Johnson ${ }^{[54]}$ in the early 1990s resulting in a spectrum that neatly depicts the diffusion coefficient $D$ on one axis in conjunction with the corresponding chemical shift on the other axis (Figure 1.7).

Clear signal separation is vital to the correct evaluation of the diffusion data as can be observed particularly well in the ${ }^{1} \mathrm{H}$-DOSY spectrum of $n \mathrm{BuLi}$ in THF presented below. At $\delta=-1.0 \mathrm{ppm}$, where the $\alpha-\mathrm{CH}_{2}$ protons resonate, a separation of the signals corresponding 
to differently sized aggregates is afforded in the diffusion dimension whereas the overlapping signal around $\delta=1.0 \mathrm{ppm}$ severely impedes the exact identification of the diffusion coefficient (vertical axis).

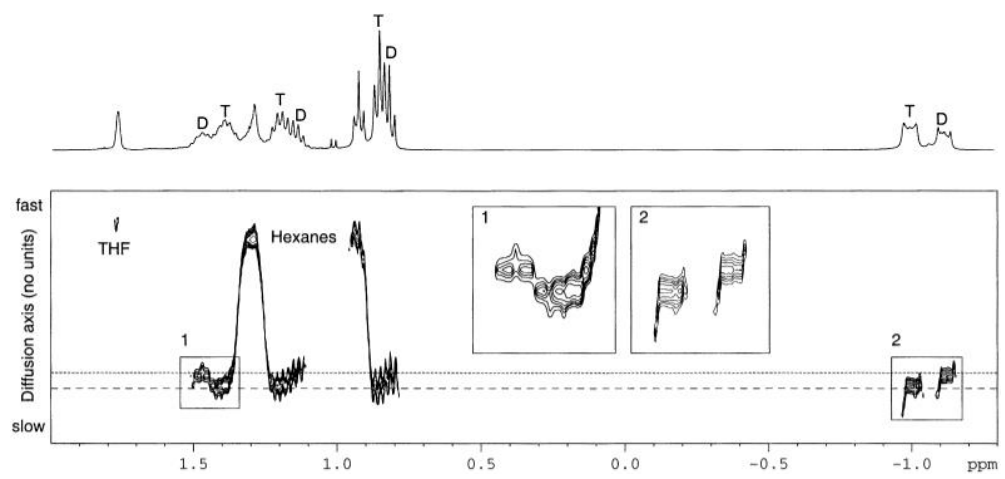

Figure 1.7: $2 \mathrm{D}$ diffusion ordered ${ }^{1} \mathrm{H}$ NMR spectrum (DOSY) of $n \mathrm{BuLi}$ in THF- $d_{8}$ at $-84^{\circ} \mathrm{C}$ and corresponding ${ }^{1} \mathrm{H}$ NMR spectrum. ${ }^{[5]}$ The butyl resonances of dimeric (D) and tetrameric (T) $n \mathrm{BuLi}$ were previously assigned.

Williard et al. utilized ${ }^{1} \mathrm{H}$ - as well as ${ }^{7} \mathrm{Li}$-DOSY experiments at low temperature to demonstrate the wide applicability of diffusion experiments for the identification and characterization of aggregation states in the case of equilibria. ${ }^{[55]}$ As a prominent example of this frequent phenomenon in organotlithium chemistry they took a closer look on an $n$ BuLi solution in THF, which has been established to contain both tetrasolvated dimeric and tetrasolvated tetrameric aggregates. Hence, faster diffusion is anticipated for the smaller dimeric aggregate whereas the major tetramer should move slower in solution. In this paper both ${ }^{1} \mathrm{H}$ and ${ }^{7} \mathrm{Li}$ diffusion coefficients were obtained with fairly good agreement (same aggregate $=$ identical diffusion coefficient) and additionally compared to calculated diffusion coefficients both from X-ray diffraction data and from gas-phase optimized structures. Thereby, both the dimeric and the tetrameric arrangement could be assigned appropriately (Figure 1.7).

The dependency on the sample conditions (temperature, concentration) hampers the direct comparison between different samples, and for exact calculations of radii from diffusion experiments these conditions would have to be precisely evaluated. Certainly, the shape of a molecule naturally influences its diffusion properties. Therefore, the StokesEinstein equation underlines that although an increase of the molar mass of a complex naturally correlates with an increase of its radius, only the radius can be unambiguously extracted from these experiments. To surpass these difficulties aiming at the determination of molar masses, a defined amount of a reference compound can be added to all investigated samples. ${ }^{[56]}$

Solid state NMR spectroscopy serving as a bridge between X-ray diffraction and highresolution NMR spectroscopy has also been frequently applied to lithium organic 
compounds yielding chemical shift information and dipolar as well as quadrupolar parameters. ${ }^{[57]}$ However, this technique will not be further discussed, because the work presented herein is primarily concerned with high-resolution NMR spectroscopic analysis in solution.

\subsection{Anisotropic NMR}

NMR spectroscopy is well-established in terms of structure elucidation which was given a résumé with a focus on lithium in the previous chapter. However, of the four main interactions, explicitly dipolar coupling, chemical shift, quadrupolar and scalar coupling, only two are directly observable in standard high-resolution NMR spectra (Figure 1.8). These two are the isotropic part of the chemical shift and the scalar coupling. The other interactions and the anisotropic, thus orientation-dependent, parts of the former are averaged to zero due to the tumbling motion of all molecules in the solvent environment. These interactions contribute to the overall relaxation of nuclear spins, which becomes apparent, for example, in case of ${ }^{7} \mathrm{Li}(I=3 / 2)$ where quadrupolar relaxation is dominant resulting in broad lines that are typical for nuclei with $I>1 / 2$.

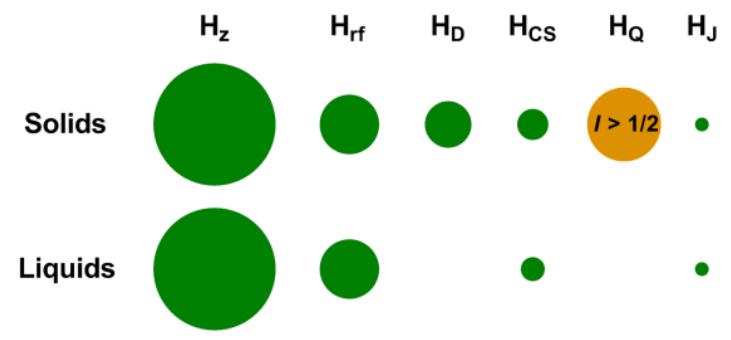

Figure 1.8: Representation of different interactions and their corresponding extent of contribution to the NMR spectrum of solids and liquids.

In contrast, dipolar relaxation gives rise to the nuclear Overhauser effect, yielding very valuable distance information. ${ }^{[58]}$ Therefore, the $3 \mathrm{D}$ structure determination of small molecules in solution can be based on the interpretation of NOE data $\left(\mathrm{r}^{-6}\right)$ and scalar couplings ( ${ }^{3} \mathrm{~J}$ coupling constants) alone. The latter allows extraction of dihedral information from the magnitude of coupling constants by the well-known Karplus relation. ${ }^{[59]}$ Certainly, these parameters are restricted by the close proximity (up to $5 \AA$ ) required for the detection of NOEs and the direct connectivity (three bonds) in case of scalar couplings. Thus, they provide only localized, short-range structural information and a method is required that does not rely on these conditions to yield long-range restraints even in fragments with disrupted NOE or J-connectivity. 
These restraints can be derived from the above-mentioned, orientation-dependent interactions which are well known in solid state NMR spectroscopy. However, the interpretation of solid state NMR spectra is hampered and complicated by dipolar and quadrupolar contacts because they are in the range of many kilohertz and, due to the presence of manifold orientations in powders, not easily extractable. ${ }^{[60]}$ Therefore, magic angle spinning (MAS) is frequently applied. However, this demonstrates that by impeding the general tumbling motion of molecules in solution, these anisotropic parameters become accessible. An environment that inhibits this motion and induces a preferred orientation (thus aligns the molecules) is called alignment medium. Liquid crystals have been known since the $1960 \mathrm{~s},{ }^{[61]}$ however investigations were mainly focused on observing the highly ordered liquid crystals themselves which was difficult due to the fact that the corresponding spectra were very complicated and only interpretable by simulation. In contrast, molecules inside these liquid crystalline systems experience only a moderate degree of alignment. This technique gained impact impetuously when Tjandra and Bax introduced the highly valuable concept of partial alignment. ${ }^{[62]}$ Under these conditions, an intermediate state between solid and liquid state is accomplished yielding smaller, "residual" anisotropic parameters. In case of dipolar couplings, for instance, the reduced magnitude of the resulting coupling being less than the scalar coupling leads to more straightforward spectra and affords the direct extraction of residual dipolar couplings (RDCs). Certainly, partial alignment and especially the application of RDCs for 3D structure determination in solution has attracted the attention of many spectroscopists and was recently reviewed by Christina $M$. Thiele ${ }^{\left[{ }^{[3]}\right.}$ and Yan et al. ${ }^{[64]}$ with a focus on small organic molecules.

\subsubsection{Alignment Techniques}

Currently, a variety of techniques for the alignment of biological and organic compounds is being established and will be summarized in the following. The attention will be drawn to liquid crystalline material and strain induced alignment in a gel (SAG) which both have been of particular importance for the analysis of small organic molecules in non-aqueous solution in recent years. Further requirements are the polarity and the stability of the medium specifically with respect to the analysed compounds and the temperature in general. The solvent deuterium quadrupolar splitting represents a general and routinely used parameter for the quality of most alignment media. Further, a classification into selfalignment (does not show any deuterium quadrupolar splitting) and external alignment media can be done. 


\subsubsection{Self-alignment}

The most fundamental alignment type is the self-alignment of molecules when exposed to a strong magnetic field. The induced alignment is rather small and proportional to the anisotropy in the magnetic susceptibility $\chi$ and to the square of the magnetic field. ${ }^{[65]}$ This minute degree of orientation has proven to yield very valuable structural information in the case of human ${ }^{15} \mathrm{~N}$-enriched ubiquitin. ${ }^{[6]}$ Bax et al. were able to illustrate the magnetic field dependence of apparent nitrogen - proton $J$ splittings (one-bond ${ }^{1} J_{\mathrm{NH}}$ couplings). One interaction held responsible for this relation is a dipolar contribution caused by a small degree of alignment due to the anisotropic magnetic susceptibility of the diamagnetic protein.

In myoglobin though, this effect is more pronounced due to the paramagnetic iron II centre. ${ }^{[67]}$ Therefore, exploitation of this phenomenon seems beneficial and can be achieved by either exchange of a diamagnetic to a paramagnetic metal ion (in case of metalloproteins $)^{[68]}$ or attachment of a special tag to the protein which then is able to bind a paramagnetic metal ion, e.g. a lanthanide. ${ }^{[69]}$ Amongst others, constraints can thus be derived from contact shifts, pseudo contact shifts and RDCs (Residual Dipolar Couplings) arising from the partial orientation of a paramagnetic metalloprotein in high magnetic fields. ${ }^{[70]}$ How powerful and advantageous this technique is in the field of protein structure elucidation becomes particularly evident for the example of a calcium binding protein $\mathrm{D}_{9 \mathrm{k}}$. The structure obtained by paramagnetic constraints is immensely more precise than the ensemble of conformers derived from the diamagnetic form (Figure 1.9).
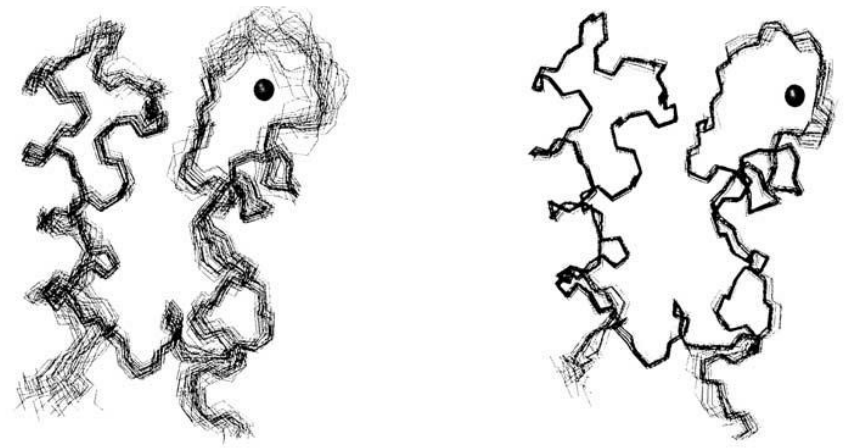

Figure 1.9: Family of 30 conformers for a calcium binding protein, calbindin $D_{9 k}$ in which one of the two calcium ions is substituted by $\mathrm{Ce}^{3+}$. Conformers were obtained by diamagnetic (left) and paramagnetic (right) constraints. $^{[71]}$

\subsubsection{Phospholipid Bicelles}

A special case of liquid crystals are so-called bicelle phases, which represent one of the oldest alignment techniques and a versatile alternative to direct field-induced alignment. In the early 1990s, Sanders et al. introduced a meanwhile frequently employed mixture of dimyristoylphosphatidylcholine (DMPC) and dihexanoylphosphatidylcholine (DHCP) 
(Figure 1.10). ${ }^{[72]}$ As schematically depicted in the same figure, an aqueous solution of DMPC and DHCP gives rise to the formation of neutral double-layered disc-shaped membranes that strongly align in the presence of a magnetic field.
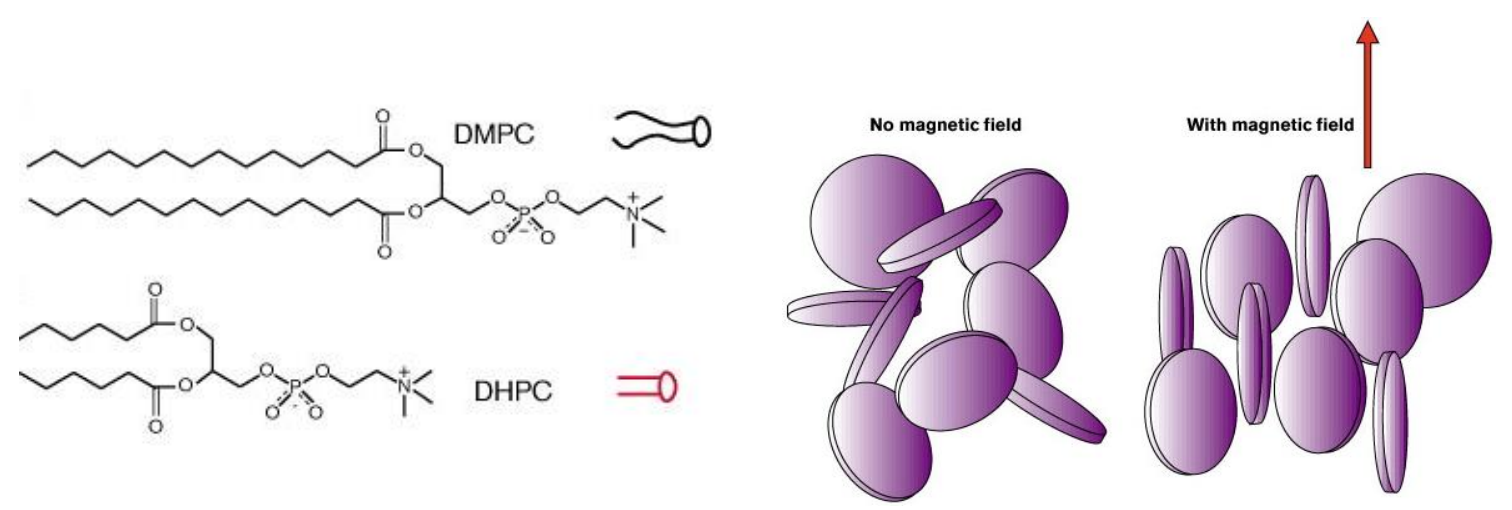

Figure 1.10: Molecular structures of DMPC and DHPC forming disc-shaped bicelles whose alignment with the magnetic field is schematically depicted on the right. ${ }^{[73]}$

Solutions of DMPC and DHPC exhibit these liquid crystalline-like ( $\mathrm{L}_{\alpha}$ phase) bilayer properties over wide ranges of water content, buffer $\mathrm{pH}$ and composition, temperature and DMPC : DHCP ratios. The bicelles represent an oriented matrix with a very high degree of order which is transferred to water-soluble molecules by exclusion of orientations due to steric interactions. The degree of alignment induced by this method is considerably higher than for (paramagnetic) single molecules as well as easily scalable and also existent at low magnetic field strengths. ${ }^{[74]}$ It thus significantly facilitates the measurement of RDCs, RQCs (Residual Quadrupolar Couplings) and RCSAs (Residual Chemical Shift Anisotropies). A separation of isotropic and anisotropic contributions is feasible and permits correlation with other isotropic parameters. Apart from studies to gain a deeper understanding of the alignment mechanism in bicelles in general, insertion of integral membrane proteins into magnetically aligned discotic phospholipid bilayers allows studying of their interactions with lipids, drugs, etc. by EPR spectroscopy. ${ }^{[75]}$

\subsubsection{Bacteriophages and Purple membranes}

A further method to induce alignment of biological macromolecules is the addition of magnetically aligned filamentous bacteriophages. Consisting of a coat protein helically packed around an ssDNA core, the bacteriophage Pf1 is very frequently applied (Figure 1.11 , left). Alignment of macromolecules by this technique represents a very stable and versatile method because the alignment strength can be modified over a wide concentration and temperature range. Furthermore, the straightforward handling and the wide availability of bacteriophages makes them highly suitable for nucleic acids and proteins. ${ }^{[76]}$ Instead of alignment induced by steric interactions as observed for bicelles, anisotropic interactions in bacteriophages are of mainly electrostatic nature. 

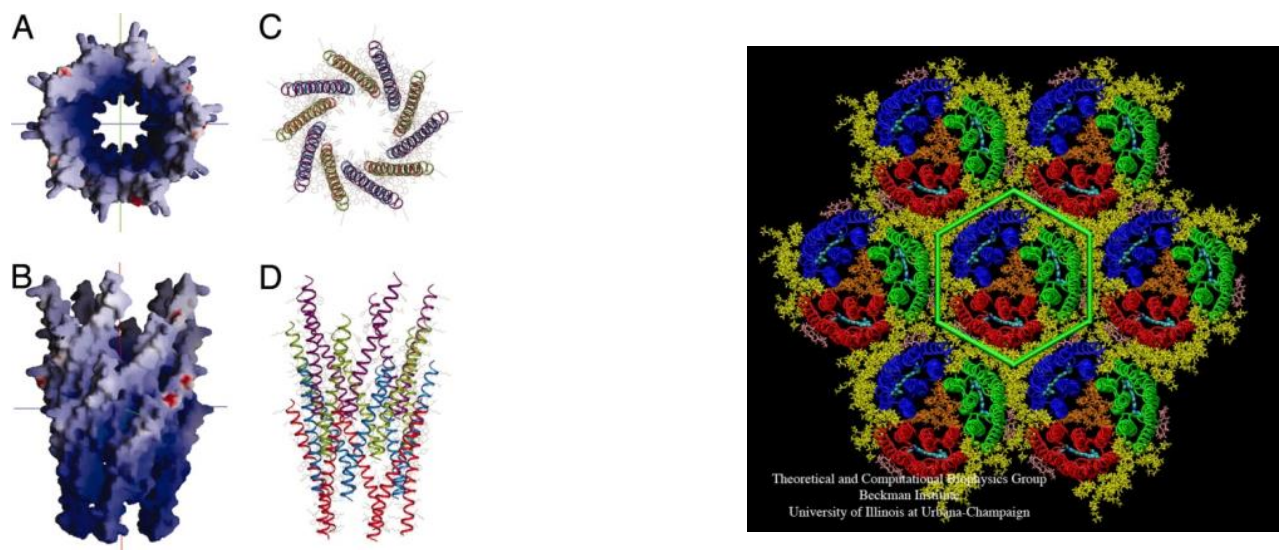

Figure 1.11: Model of a section of the Y21M fd filamentous bacteriophage capsid (similar to the commonly applied Pf1 bacteriophages) built from the coat protein subunit structure, which was determined by solidstate NMR spectroscopy (left $)^{[77]}$ and top view of a purple membrane patch that includes 21 bacteriorhodopsins in seven adjacent unit cells. ${ }^{[78]}$

Bacteriorhodopsin in contrast is a transmembrane protein functioning as light sensor and proton pump. In the purple membrane, trimers of bacteriorhodopsin are arranged hexagonally (Figure 1.11, right). The alignment of water-soluble biomacromolecules is mainly dominated by electrostatic interactions, so that only small concentrations of the purple membrane are required. Although this alignment medium is very stable, interaction of the analyte with the charged membrane leads to a decrease of the transverse relaxation time and thus considerably broadened spectra. ${ }^{[79]}$ Thus, suitable molecules are most likely proteins at $\mathrm{pH}$ values above their isoelectric point and the negatively charged nuclei acids.

\subsubsection{Liquid Crystals for Small Organic Molecules}

Due to the massive impact on the structure determination of biological molecules, a variety of alignment media has been developed as shortly summarized in the preceding subchapters. However, in the case of small organic molecules these methods cannot be employed as they all work on the basis of aqueous solutions. Nowadays, two strategies for the alignment of organic molecules exist, one being the utilization of liquid crystals

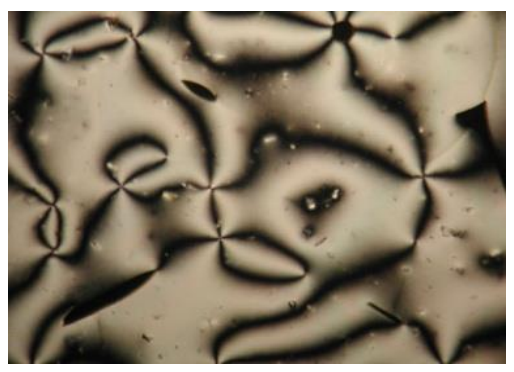

Figure 1.12: Schlieren texture of the nematic phase of liquid crystals. ${ }^{[80]}$ described in this chapter, the other - stain induced alignment in a gel - is described later on. Liquid crystals (LC), simultaneously displaying characteristics of liquids as well as solid crystalline materials, were first introduced by Saupe et al. in the 1960s. ${ }^{[6]]}$ By then, mainly nematic phases of the liquid crystals itself (Figure 1.12) were analysed. The analysis of the resulting spectra was complicated and often only afforded by simulations. 
To determine anisotropic NMR parameters an alignment is induced by transferring the high degree of order of the liquid crystal to small molecules. For the purpose of yielding less complicated spectra that could be interpreted more unambiguously a small degree of alignment $\left(10^{-3}\right)$ is beneficial. Courtieu and co-workers presented the VASS (Variable Angle Sample Spinning) method to obtain less complex spectra. Thereby, the sample is rotated similar to MAS (Magic Angle Spinning) in solid state NMR, and by choice of the spinning angle the observed anisotropic parameters can be scaled down by a known factor. ${ }^{[81]}$ However, this requires the adequate hardware which is why nowadays lyotropic phases are utilized, which allow tuning of the alignment strength by varying the liquid crystal concentration. LC consist of at least two components: a "liquid crystal builder" and a solvent. ${ }^{[82]}$ Apart from composites of $\mathrm{C}_{12} \mathrm{E}_{5}$ with $n$-hexanol in $\mathrm{D}_{2} \mathrm{O}$ or $\mathrm{D}_{2} \mathrm{O} / \mathrm{DMSO}$ mixtures, ${ }^{[83]}$ homopolypeptides are an important class of liquid crystal builders and in addition also compatible with a wide variety of organic solvents $\left(\mathrm{CDCl}_{3}, \mathrm{C}_{6} \mathrm{D}_{6}, \mathrm{THF}\right.$, Dioxane, DMF). The application of these homopolypeptides has been promoted by Courtieu, Lesot and co-workers who employed a chiral liquid crystal phase composed of poly- $\gamma$-benzyl-L-glutamate (PBLG) dissolved in an organic solvent. The chiral nature of PBLG also permits enantiodiscrimination (Figure 1.13). ${ }^{[84]}$ Apart from PBLG, other homopolypeptides like poly- $\gamma$-ethyl-L-glutamate (PELG) and poly- $\varepsilon$-carbobenzyloxy-Llysine (PCBLL) have been investigated and frequently applied. ${ }^{[85]}$
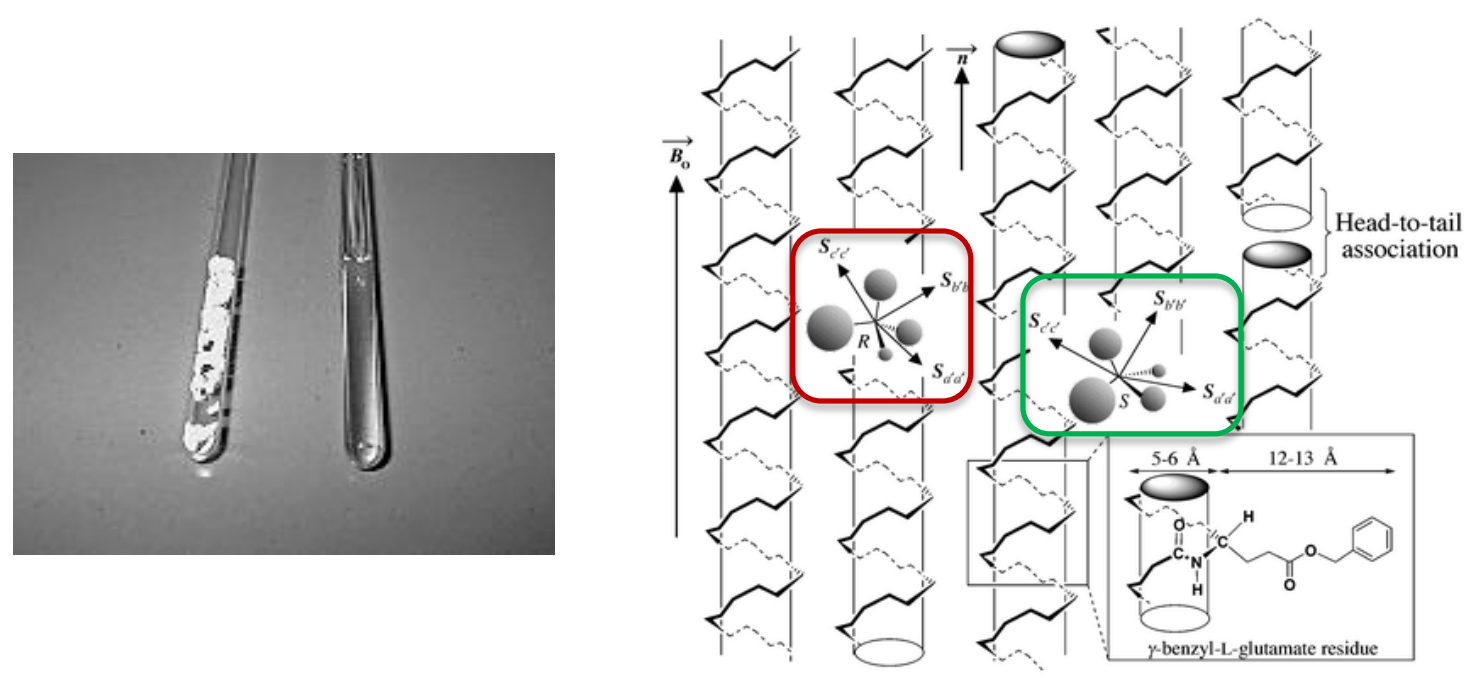

Figure 1.13: NMR sample tubes $(5 \mathrm{~mm})$ with solid $\mathrm{PBLG}$ in $\mathrm{CDCl}_{3}$ and after the formation of the lyotropic liquid crystalline phase. The differential ordering effect for two enantiomers dissolved in the PBLG phase is schematically depicted on the right (red and green boxes). The difference of interaction between the enantiomers and the PBLG molecules produces a measurable difference in their orientational ordering. The glutamate side chains of the polypeptide and the co-solvent molecules are not displayed. ${ }^{[63 b, 84 a]}$

The induced alignment in PBLG liquid crystals may be readily tuned by either changing the temperature or the concentration of the sample. However, the dilution is limited by a threshold concentration below which the ordered sample will directly give way to an 
isotropic solution which nevertheless is reversible upon evaporation of the excess solvent material. Changing to aforementioned PELG and PCBLL phases as well as to the newly investigated polyacetylenes might also result in a smaller degree of alignment. ${ }^{[86]}$ Thiele et al. focused further on the optimization of the most prominent PBLG liquid crystal phase in order to tune it with respect to its critical concentration, preferred orientation and enantiodifferentiation capacity. ${ }^{[87]}$ By varying the molecular weight (MW) of the PBLG material, they could remarkably verify that high-MW PBLG affords notably lower critical concentrations (about 6\%) and thus a lower degree of order along with significantly improved spectral quality. Furthermore, the utilization of high-MW PBLG allows the addition of DMSO to $\mathrm{CDCl}_{3}$ up to a ratio of 1:2 while still retaining the liquid crystalline phase. Another beneficial, highly non-polar additive is $\mathrm{CCl}_{4}$ that is able to continuously lower the degree of order with increasing $\mathrm{CCl}_{4}$ concentration (more pronounced aggregate formation with PBLG) and besides has an advantageous effect on the degree of enantiodiscrimination. These findings underline and enhance the wide applicability of homopolypeptides as alignment and enantiodiscrimination media.

\subsubsection{Strain Induced Alignment in a Gel (SAG)}

Strain induced alignment in a gel (SAG) is an approach that is conceptually different from the application of liquid crystals. To induce order, SAG samples are prepared by swelling polymer sticks in deuterated solvents. Their expansion is hindered, e.g. by the glass walls of an NMR sample tube. If the diameter of the employed tube is smaller than the equilibrium diameter of the fully swollen gel, a uniaxially anisotropic strain is induced (Figure 1.14). Thereby, the cavities inside the gel are being deformed to ellipsoids, in which both solvent and dissolved compound will adapt on average an orientation in which the long axis of the molecule will be parallel to the magnetic field just like in lytropic or bicelle phases. In contrast to liquid crystals however, SAG does not rely on the magnetic field as activator of the alignment.

Samulski and Deloche introduced strained elastomers to induce order in solvents, which were analysed by ${ }^{2} \mathrm{H}$ NMR spectroscopy, which now functions as a benchmark for the medium's quality. The observation of clean quadrupolar doublets $(I=1$, see also chapter 1.2.2.2) with regular line shape accounts for a homogeneously swollen polymer gel. Poly(dimethylsiloxane) (PDMS) and its swelling behaviour was also investigated. However, those NMR spectroscopic studies mainly focused on the characterization of the polymer network itself rather than on the alignment induced on other, dissolved compounds. ${ }^{[88]} \mathrm{Luy}$ et al. investigated many polymeric gels for their alignment properties in a cutting-edge 
manner. ${ }^{[89]}$ Of those, cross-linked polystyrene networks are chosen to exemplarily illustrate the applications and limitations of polymer gels as alignment medium.
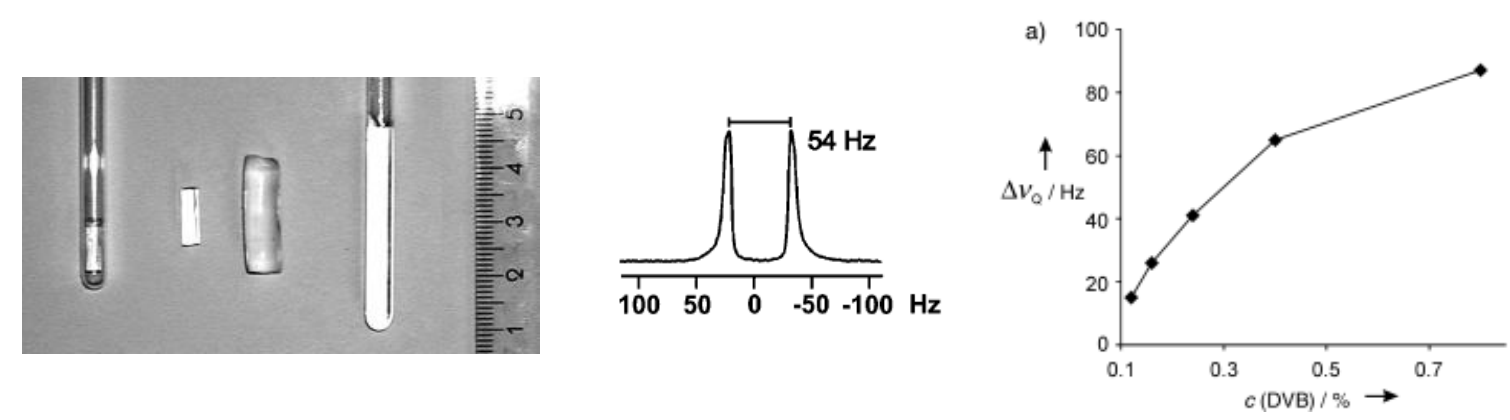

Figure 1.14: Cross-linked PS sticks in different states of swelling (left), which show a typical quadrupolar splitting of $\mathrm{CDCl}_{3}$ (middle). This quadrupolar splitting $\Delta v_{\mathrm{Q}}$ in the deuterium signal of $\mathrm{CDCl}_{3}$ depends on the DVB concentration (right). ${ }^{[896]}$

Cross-linked polystyrene can be produced in a straightforward manner by polymerizing degassed styrene with divinylbenzene as linker and the radical starter AIBN (2,2'-azobis(2methylpropionitrile)) or DBP (dibenzoyl peroxide) in glass tubes of defined diameters. The homogeneity of the swollen gels and the accomplishing of the equilibrium position can be nicely monitored by the quadrupolar splitting in the ${ }^{2} \mathrm{H}$ NMR spectrum. Polystyrene gels can be obtained by swelling in various solvents which require different swelling times due to polarities and the diameter of the polymer stick. The analyte is then added on top of the gel and diffuses into the already swollen gel. However, although the gel is quite inert and thus the scope of compounds to be analysed quite large, very polar solvents like DMSO or acetone do not lead to the formation of orienting gels. This limits the spectrum of compounds to those analytes that are soluble and stable in the given solvents $\mathrm{CDCl}_{3}$, $\mathrm{CD}_{2} \mathrm{Cl}_{2}, \mathrm{C}_{6} \mathrm{D}_{6}$, THF and Dioxane (Table 1.2). Luy and co-workers observed the overall alignment to be independent of the magnetic field strength and observable over a wide temperature range $(215-325 \mathrm{~K})$. Figure 1.14 further depicts a clear tendency of the quadrupolar splitting of the solvent's ${ }^{2} \mathrm{H}$ NMR signal with respect to the cross-linker concentration. An increasing amount of junction points logically results in smaller cavities which imply a more pronounced preferred orientation of the molecules inside the gel. Therefore, the degree of order is higher which in turn is represented by a higher quadrupolar splitting. This means that by choice of a different degree of cross-linking the magnitude of the observed parameters might be scaled. The same holds true for the diameter of the polymer stick and the solvent system. Change of the latter might also cause a different alignment of the analytes. ${ }^{\left[{ }^{[9 d]}\right.}$ Certainly, all variations have to be implemented in a new sample preparation. The interpretation of the anisotropic spectra is only impeded by 
the huge polymer signals which can be alleviated either by using deuterated polymers or pulse sequences that suppress these signals. ${ }^{[90]}$

Meanwhile, numerous other polymer systems serving as alignment media have been developed. They vary in polarity and therefore in the solvents which allow them to swell (Table 1.2).

Table 1.2: Selected available orienting media and their compatibility with organic solvents. Differentiation into compatible, non-compatible and not stated by the authors is implemented.

\begin{tabular}{|c|c|c|c|c|c|c|c|c|c|c|c|}
\hline & $\begin{array}{l}\mathrm{CDCl}_{3} \\
\mathrm{CD}_{2} \mathrm{Cl}_{2}\end{array}$ & $\mathrm{C}_{6} \mathrm{D}_{6}$ & Hexane & THF & Dioxane & DMF & $\mathrm{CD}_{3} \mathrm{OD}$ & $\mathrm{CD}_{3} \mathrm{CN}$ & Acetone & DMSO & Refs. \\
\hline PS & $\checkmark$ & $\checkmark$ & $x$ & $\checkmark$ & $\checkmark$ & $?$ & $?$ & $x$ & $x$ & $x$ & {$[89 \mathrm{~b}, 89 \mathrm{~d}]$} \\
\hline PDMS & $\checkmark$ & $\checkmark$ & $\checkmark$ & $\checkmark$ & $\checkmark$ & $?$ & $?$ & $?$ & $?$ & $x$ & [89a] \\
\hline PMMA & $\checkmark$ & $?$ & $?$ & $?$ & $?$ & $?$ & $?$ & $?$ & $?$ & $?$ & [91] \\
\hline PVAc & $\checkmark$ & $\checkmark$ & $?$ & $\checkmark$ & $\checkmark$ & $?$ & $\checkmark$ & $\checkmark$ & $\checkmark$ & $\checkmark$ & [89c] \\
\hline PAA & $?$ & $?$ & $?$ & $?$ & $?$ & $\checkmark$ & $?$ & $?$ & $?$ & $\checkmark$ & [92] \\
\hline PAN & $x$ & $?$ & $?$ & $?$ & $?$ & $x$ & $x$ & $?$ & $?$ & $\checkmark$ & [89e] \\
\hline
\end{tabular}

While the polystyrene (PS) gels presented in detail in the two preceding pages are not compatible with completely non-polar and very polar solvent environments, poly(dimethylsiloxan) (PDMS) gels also swell in non-polar solvents like $n$-hexane. In turn, poly(methyl methacrylate) (PMMA), polyacrylamide (PAA also $\mathrm{PH}$ ) and poly(acrylonitrile) (PAN) gels are less investigated with respect to their solvent compatibility. PAA is also tuneable by copolymerization with acrylates yielding charged gel environments. ${ }^{[93]}$ Polyvinylacetate (PVAc) possesses the biggest amount of matches both for polar and non-polar solvents. Furthermore, alignment media based on collagen and gelatine are available, but applied almost exclusively to aqueous systems. The polymer gel has to be carefully chosen by the user with respect to the analyte's properties like polarity, reactivity etc.

Instead of strain building up intrinsically during swelling, the strain can also be induced by stretching or compressing the swollen gel with the help of an external apparatus. Several devices for both purposes have been developed and represent a further screw that might be turned in order to tune the induced degree of order. ${ }^{[94]}$ 


\subsubsection{Residual Anisotropic NMR Parameters (RDCs, RQCs and RCSAs)}

\subsubsection{Residuals Dipolar Couplings (RDCs)}

Between two spins with permanent magnetic dipole moments, dipole - dipole interactions can be observed. The strength of the direct dipole - dipole interaction for two isolated (heteronuclear) spins I and S strongly depends on the distance (inverse third power) between the nuclei and their relative orientation. It can be defined by the following equation $(1-3) \cdot{ }^{[95]}$

$$
D_{\mathrm{IS}}=-\frac{\mu_{0}}{4 \pi} \frac{\hbar \gamma_{\mathrm{I}} \gamma_{\mathrm{S}}}{2 \pi r_{\mathrm{IS}}^{3}}\left(3 \cos ^{2} \theta-1\right)
$$

Therein, $\gamma$ represents the magnetogyric ratio of the corresponding spins, $\mu_{0}$ the vacuum permeability and $\theta$ the angle between the connecting IS-vector and the magnetic field $B_{0}$ (Figure 1.15). The angular and distance dependency makes dipolar interactions a powerful tool for structure elucidation. In solid state NMR, this phenomenon is very important, e.g. in powder spectra all orientations of the molecule are equally populated with respect to $B_{0}$ and a so-called pake doublet is observed. In solution however, this orientation constantly changes due to the Brownian diffusion. Thereby, the angle-dependent term in brackets is averaged over time yielding an overall cancellation of dipole interactions in isotropic

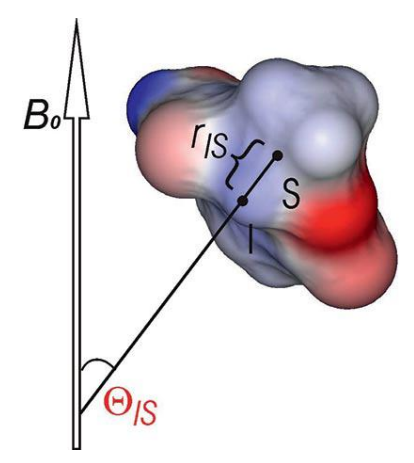

Figure 1.15: Representation of a rigid molecular fragment containing the spins I and $S .{ }^{[63]}$ solution.

If a molecule is now incorporated into one of the various available alignment media, a preferred orientation at the expense of sterically or electronically unfavourable arrangements is induced. By doing so, dipolar couplings can be obtained serving as powerful global restraints for structure determination. These couplings are obtained in a straightforward manner, yet their value is substantially smaller than that in the solid state. Therefore, the term "Residual Dipolar Couplings" (RDCs) is selected to account for this partial orientation.

If the degree of orientation is considerably small $\left(10^{-4}\right.$ to $\left.10^{-3}\right)$ and thus the measured dipolar couplings are less than the scalar couplings, the same experiments may be accomplished to extract those parameters along with the identification of the sign of the dipolar interaction, e.g. in the case of $\mathrm{CH}$-couplings (equation (1-4)) 


$$
{ }^{n} T_{\mathrm{CH}}={ }^{n} J_{\mathrm{CH}}+{ }^{n} D_{\mathrm{CH}}
$$

In this equation $T$ represents the overall coupling observed between a certain $\mathrm{C}$ - and $\mathrm{H}$ atom and $n$ the number of bonds separating both nuclei. $J$ stands for the scalar coupling and $D$ implies the dipolar contribution. For this reason, the dipolar part of the total coupling can be determined straightforwardly by comparing the total coupling obtained by both isotropic (where $D=0$ ) and anisotropic measurements. While the first only consists of the scalar coupling, the latter is assembled by addition of the scalar and the dipolar contribution. For accurate determination of carbon-proton RDCs an increasing number of experiments is now available.

In the following, the application of RDCs to the determination of three-dimensional structures will be discussed with a focus on the benefit for small molecules. Hereby, either structure refinement by fitting RDC data to a number of structure proposals or assignment of the signals to a molecule with known structure is sought.

A conceptually very straightforward utilization of RDCs was promoted by Shapiro et al. for the determination of the relative stereochemistry of six-membered rings. ${ }^{[96]}$ The direction of the vector connecting I and S is not directly measurable; only the angle towards the magnetic field is extractable. Therefore, parallel vectors - independent of the direction which they point in - should display identical RDCs. This was promoted in the abovementioned publication in order to distinguish between axial protons (all parallel and with similar sized RDCs) and equatorial ones which lack this geometrical relation in the model molecule 4,6-O-ethylidene-D-glucopyranose (Figure 1.16).
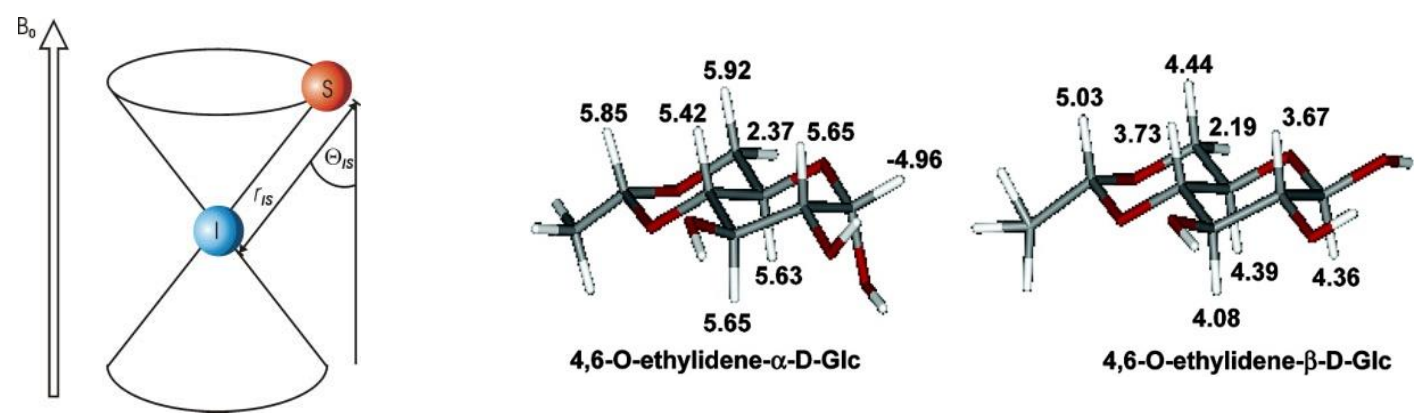

Figure 1.16: Cone-shaped representation of the possible orientations of a vector connecting the spins I and $S$ belonging to given a RDC at a distance $r$ (left). A map of $\mathrm{C}-\mathrm{H}$ dipolar couplings on the structures of $\alpha$ - and $\beta$ 4,6-O-ethylidene-D-glucopyranose isomers is depicted on the right.

A further example that demonstrates the utility of RDCs to yield structural assignment is depicted in Figure 1.17, which shows the results obtained for the fitting of RDCs to strychnine in case of correct assignment (black) and misassignment of one pair of diastereotopic protons (red). 


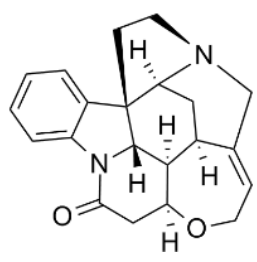

strychnine
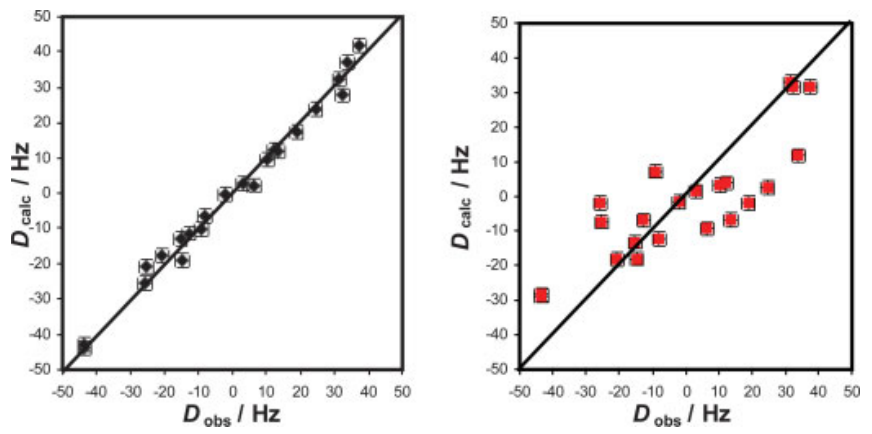

Figure 1.17: Comparison of observed ${ }^{1} \mathrm{D}_{\mathrm{C}-\mathrm{H}}\left(\mathrm{D}_{\mathrm{obs}}\right)$ and for a given structure back-calculated $\mathrm{RDCs}\left(\mathrm{D}_{\text {calc }}\right)$ for the assignment of all diastereotopic groups (left, black) and a misassignment of one single pair $\left(\mathrm{H}_{11 \mathrm{a}}\right.$ and $\left.\mathrm{H}_{11 \mathrm{~b}}\right)$ of diastereotopic protons (right, red) in strychnine. ${ }^{[63 a]}$

If all protons are assigned correctly a straight line results from the fitting process (black). Thereby, the experimentally observed RDC values are plotted against those derived from calculations based on the strychnine structure. The root mean square deviation is then a criterion for the quality of the diastereotopic assignment. If however, the calculated RDCs vary significantly from the experimental ones (red marks) the assignment does not match with the experimental data. In this case, the misassignment of one single pair of diastereotopic protons causes considerably large deviations for the entire strychnine molecule.

If structure determination is aimed, the first and by far most often used method is fitting of the RDC data to several structure proposals. If five independent (not parallel) RDCs can be obtained for a molecule, its alignment tensor (orientation of the molecular coordinate frame with respect to the magnetic field) can be calculated. In cases in which more than five independent RDCs are accessible they can be fitted to different structure proposals for a given molecule. A good agreement between experimental and calculated RDCs confirms the proposed arrangement to be an adequate representation of the actual structure in solution. The second approach presented if structure validation is aimed is most beneficial if a large number RDCs for a given analyte is accessible. This enables to use one set of RDCs (for example, of one part of the molecule) for the calculation of the alignment tensor of the whole molecule. The known alignment tensor is then employed in order to predict values for the remaining RDCs which can afterwards be compared to the experimental ones.

One of the very few organometallic complexes analysed by anisotropic NMR is a catalystsubstrate complex of a palladium catalyst which displays high enantioselectivity in allylic alkylation reactions (Figure 1.18). ${ }^{[97]}$ At the same time, this investigation particularly underlines the potential of a combination of the "standard" local restraints (J, NOE) and RDCs as global restraints. In a first approach to determine the conformation in solution 
and thus understand the enantioselectivity of the given catalyst, possible catalyst-substrate conformations were computed. In a next step, intramolecular distances were determined from NOE measurements. This did not single out one of the conformers but resulted in a preselection of three possible complex conformations that did not violate the NOE-based restraints (Figure 1.18). To explain, whether one of the three remaining conformations is preferred or if possibly an interconversion takes place in solution, RDCs were measured by aligning the sensitive molecule in high-molecular-weight PBLG under Schlenk conditions.

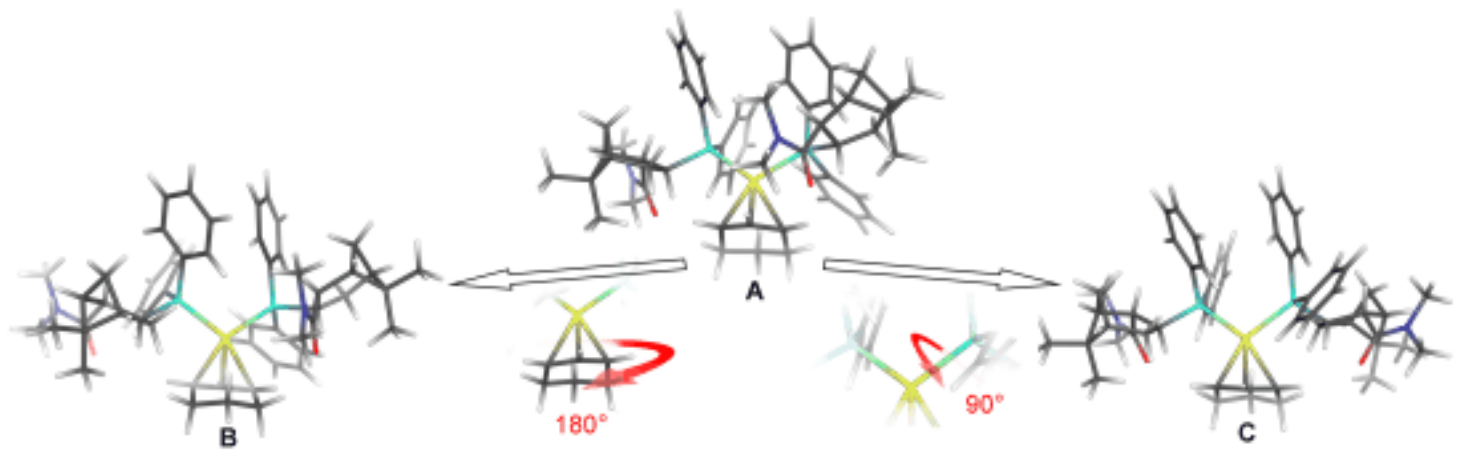

Figure 1.18: Diastereomorphous complex conformations that do not violate NOE restraints. Conformers $\mathbf{B}$ and $\mathbf{C}$ are obtained from A by either a $180^{\circ}$ turn of the cyclohexenyl substrate $(\mathbf{B})$ or a roughly $90^{\circ}$ rotation around the Pd-P bond $(\mathbf{C}){ }^{[97]}$

The measured RDCs fit best to structure A. However, the root mean square deviation is considerably high which the authors explains with a high conformational flexibility of the cyclohexyl ring. Thus, they were not only able to evaluate the conformation present in solution but they were also able to obtain values for the small energy difference between the chair and boat conformation of the cylcohexyl ring in $\mathbf{A}$ by an experimentally derived ratio of $57 \% \mathbf{A}^{\text {chair }}$ to $43 \% \mathbf{A}^{\text {boat }}$. This study very much underlines that RDCs are a very powerful tool for structure elucidation, even more so in combination with other experimental parameters. Their value for the characterization of small molecules is underlined by several studies yielding clarification of constitution and conformation often inaccessible by conventional methods. ${ }^{[98]}$ Currently, extensive investigations likewise addressing enantiodiscrimination as well as molecular flexibility are carried out by anisotropic NMR experiments.

\subsubsection{Residual Quadrupolar Couplings (RQCs)}

Nuclei with a spin quantum number $I>1 / 2$ have an electric quadrupole moment $Q$ indicating that their electric charge distribution is not spherically symmetric. These socalled "quadrupolar nuclei" are quite common and comprise $74 \%$ of all NMR active nuclei (Figure 1.19). ${ }^{[99]}$ 


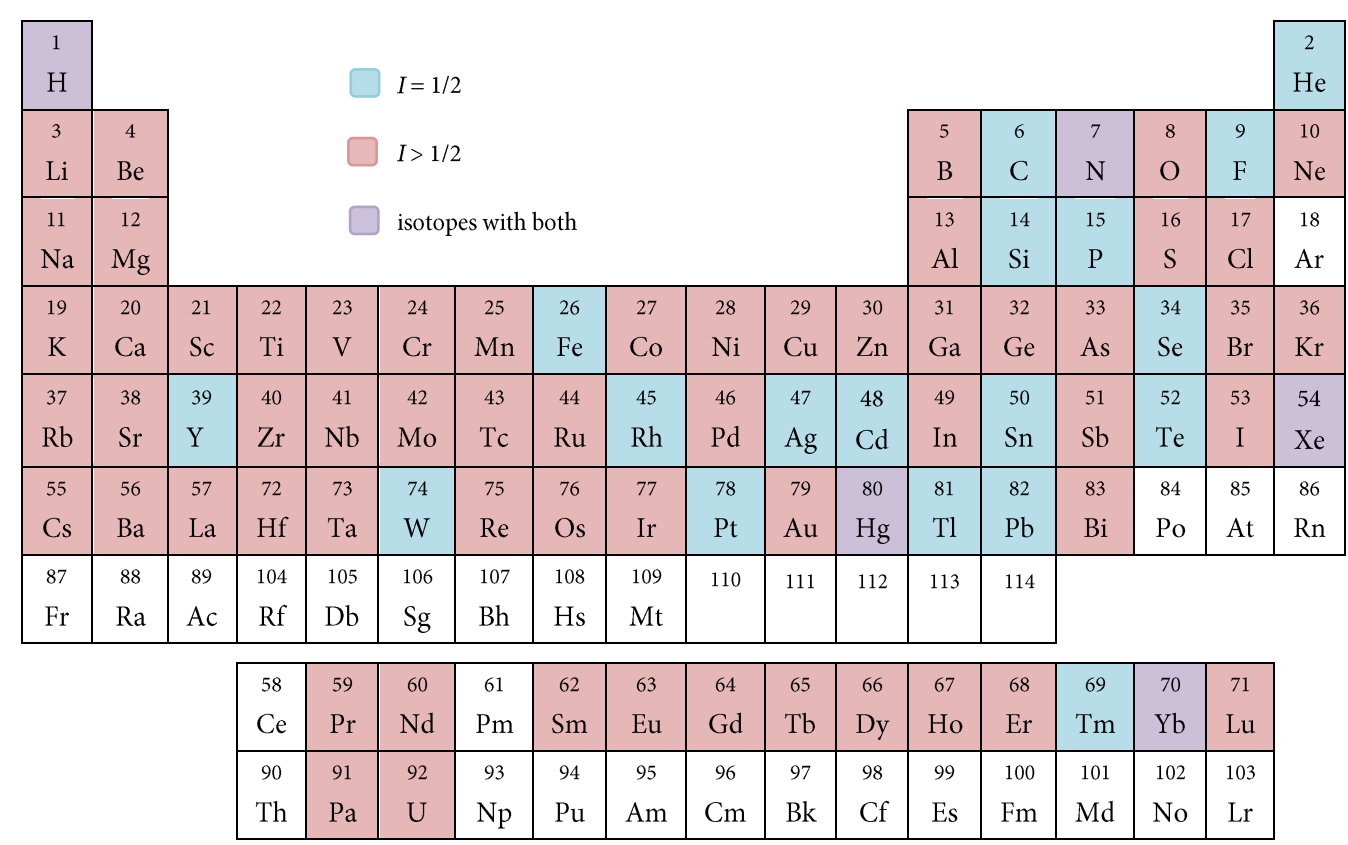

Figure 1.19: Periodic table of elements including a colour code to characterize elements according to the spin quantum number of their isotropes: blue: $I=1 / 2$, red: $I>1 / 2$ and violet: elements with isotopes of both categories.

The interaction between $Q$ and the electric field gradient (efg) produced by a nonsymmetric electronic environment of the nucleus leads to quadrupolar coupling. Here, the electric quadrupole moment is a scalar nuclear property, whereas the field gradient is a molecular attribute and a tensor quantity (V). The interaction, like dipolar coupling, depends on the molecular orientation (Figure 1.20) and averages to zero in isotropic solution due to the tumbling motion of the molecules in case of a traceless tensor.

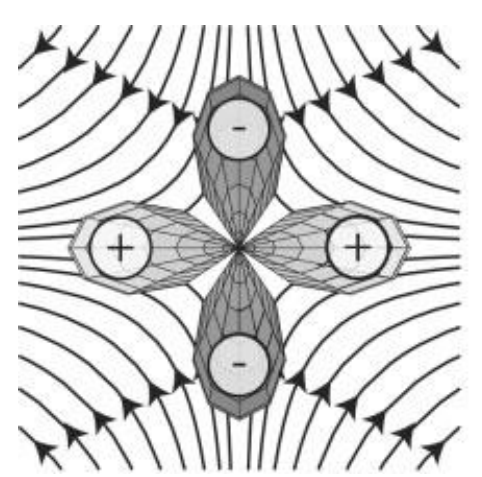

lowest energy

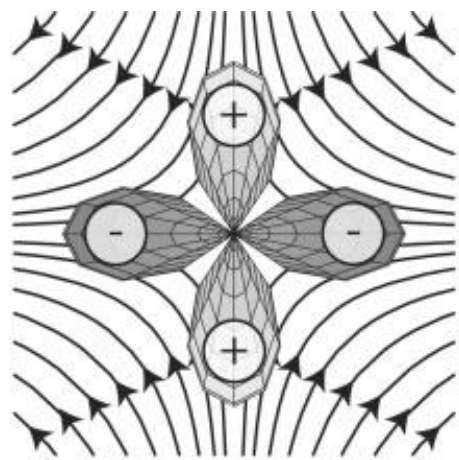

highest energy

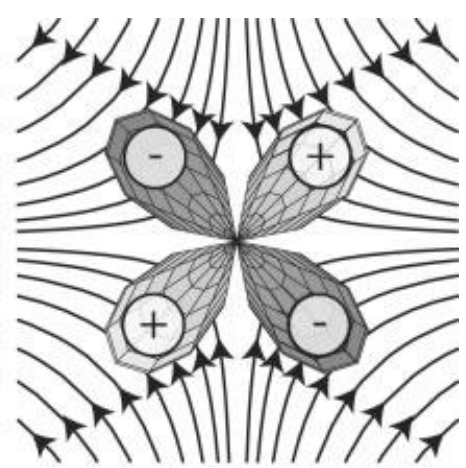

intermediate (most torque)

Figure 1.20: Schematic representation of the interaction of an electric quadrupole with an electric field gradient. The lines and arrows represent the nonuniform component of the electric field, showing the direction in which a positive charge would move. The lowest energy arrangement is when the light (positive) lobes are as far along the field lines as possible, and the dark (negative) lobes are as close as possible to the source of the field lines. ${ }^{[100]}$

As mentioned above, the electric field gradient is a molecular property, because it depends on the local symmetry, dynamics and short-range structural properties (coordination 
number, bond lengths, and bond angles). ${ }^{[101]}$ Therefore, it represents a very important indicator for the charge distribution which fortunately is accessible by different approaches. The efg tensor can be determined by solid state NMR spectroscopy if $Q$ is known while the charge distribution is accessible by means of quantum-mechanical calculations. ${ }^{[102]}$

The quadrupolar coupling which gives rise to a splitting into $2 I$ lines, if the molecule is weakly aligned along the magnetic field axis (Residual Quadrupolar Coupling (RQC)), can be described by the following equation. ${ }^{[99]}$

$$
\Delta v_{Q}=\frac{e Q}{2 h} \sum_{i, j=x, y, z} A_{i j} V_{i j}
$$

Where $e$ is the elementary charge, $h$ Planck's constant, $A_{\mathrm{ij}}$ and $V_{\mathrm{ij}}$ are the components of the alignment and efg tensors in the molecular axis system x,y,z. The formula implies that in molecules which are spherically shaped $\left(A_{\mathrm{ij}}=0\right)$ or contain a quadrupolar nucleus in a highly symmetric environment $\left(V_{\mathrm{ij}}=0\right)$ no splitting is expected.

Following the $2 I$-rule, splitting into a triplet is observed in case of nuclei with $I=3 / 2$ (e.g. $\left.{ }^{7} \mathrm{Li},{ }^{11} \mathrm{~B},{ }^{23} \mathrm{Na}\right)$. The reason for the appearance of a quadrupolar triplet is schematically depicted on the right (Figure 1.21). Inserting a spin-3/2 nucleus into a magnetic field results in a separation of the otherwise degenerated energy levels. However, in the absence of the quadrupolar interaction all transitions are equal in energy and one single line would be observed in the spectrum. Adding now the contribution due to the quadrupole interaction leads to a perturbation of the energy levels so that the low energy satellite transition is lowered in frequency by the same amount as the high energy satellite transition is raised. Therefore, three different resonances (2I) are observed for nuclei with

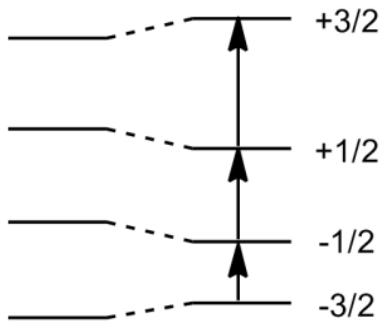

Figure 1.21: Schematic diagram of the energy levels of a spin $3 / 2$ nucleus in a magnetic field (left) and a small quadrupole perturbation (right). ${ }^{[103]}$ $I=3 / 2$ (or seven lines for ${ }^{133} \mathrm{Cs}$ with $I=7 / 2$, respectively).

The multiplet structure for different spin quantum numbers $I$ has been extensively investigated by Kuchel et al. by varying the degree of alignment for quadrupolar metal ions in aqueous gelatine. ${ }^{[104]}$ For this purpose, the authors developed a silicone rubber tube which allows facile scaling of RQCs by varying the amount of stretching. This is exemplarily illustrated for sodium $(I=3 / 2)$ (Figure 1.22): 


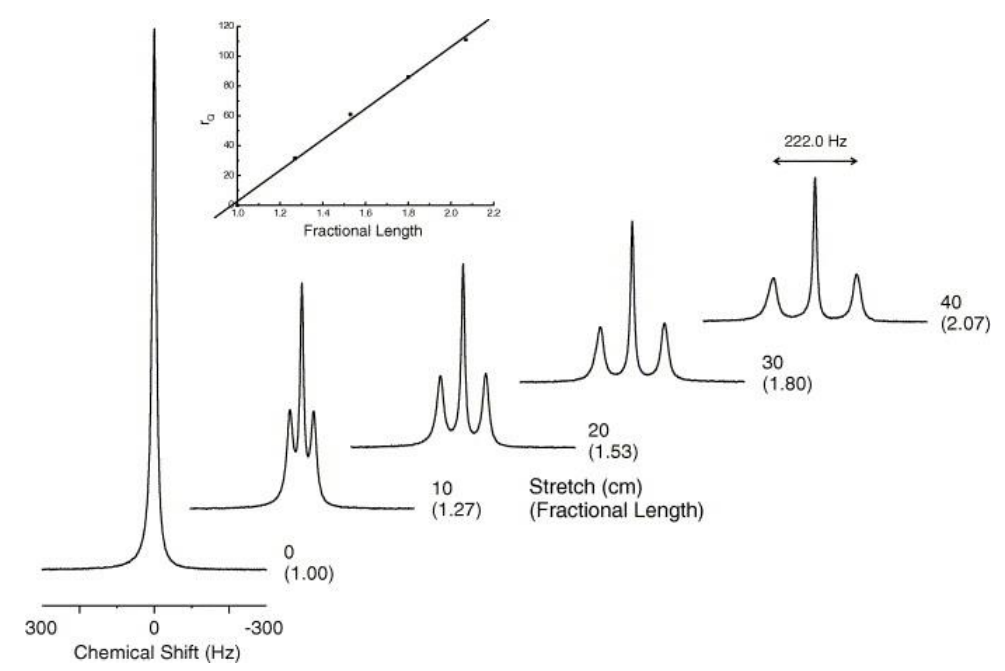

Figure 1.22: ${ }^{23} \mathrm{Na} \mathrm{NMR}$ spectra of $\mathrm{NaCl}$ in gelatine in the silicone tube under various extents of stretching (length change in $\mathrm{cm}$ and fractional length). The double arrow indicates the separation in $\mathrm{Hz}$ between the two outer components of the triplet; i.e., the value is twice the residual quadrupolar coupling constant, RQC. ${ }^{[104]}$

With zero stretching a Lorentzian-shaped resonance for $\mathrm{NaCl}$ can be observed. By increasing the stretching of the gelatine gel strain is induced which partially aligns the analysed ions. This gives rise to a quadrupolar triplet displaying a particular quadrupolar splitting constant for each corresponding extent of stretching. The observed integral ratio of 3:4:3 for the discussed triplet has also been illustratively presented by Kuchel and coworkers as result of a consecutive net transfer of 3, 4 and 3 spins during relaxation. ${ }^{[105]}$ As quadrupolar couplings are very sensitive indicators of the geometry and arrangement in the proximity of quadrupolar nuclei, they already represent a very potent tool for the solid state structure elucidation in many fields of chemistry ranging from catalysts, ${ }^{[06]}$ metalloproteins, ${ }^{[107]}$ porous material ${ }^{[108]}$ and organic polymers ${ }^{[109]}$ to ion transport in batteries. $^{[110]}$

\subsubsection{Residual Chemical Shift Anisotropies (RCSAs)}

The residual chemical shift anisotropy (RCSA) is the change in chemical shift when switching from isotropic solution to an orienting medium. In contrast to RDCs which provide information on the orientation of internuclear bond vectors (see 1.2.2.1), RCSAs yield information on the orientation of the chemical shielding tensor of individual atoms in a molecule. ${ }^{[11]}$ This information can then be employed either as constraints for structure determination or to evaluate the quality of a structure (e.g. derived from RDCs). ${ }^{[12]}$ The chemical shift $\delta$ can be represented by the following equation: ${ }^{[13]}$

$$
\sigma=\sigma^{i s o}+\sum_{i, j=x, y, z} \sigma_{i j}^{a n i s o} A_{i j}
$$


The overall chemical shift is composed of an isotropic part $\sigma^{\text {iso }}$ and an anisotropic part represented by $\sigma^{\text {aniso }} A_{\mathrm{ij}}$. $A$ is the alignment tensor which vanishes for isotropic samples. With $A=0$ the chemical shift can be described by the isotropic part alone. In case of partially aligned molecules, the orientationdependent part can be obtained in a straightforward manner by subtracting the isotropic chemical shift from the overall

Figure 1.23: Superimpostion to extract $\Delta \delta\left(\right.$ here $\left.{ }^{15} \mathrm{~N}\right) .^{[114]}$ anisotropic one (Figure 1.23).

Chemical shifts are certainly very sensitive to the sample conditions and the modification of the surroundings when applying the alignment medium produces further signal shifts. ${ }^{[89 d, 111]}$ Therefore, a method enabling the determination of both parameters in one sample is highly beneficial. Attempts to do so are the application of VASS (variable angle sample spinning), VA (variable angle experiments) or the utilization of liquid crystalline phases which can be converted from isotropic to anisotropic conditions by varying the temperature (requiring a temperature sensitive reference compound). The size of RCSAs, for example in the case of carbon, where they can be easily extracted from protondecoupled ${ }^{13} \mathrm{C}$ NMR spectra, is generally higher for aromatic moieties than for aliphatic ones due to larger $\Delta \sigma_{\mathrm{ij}}$-values. 


\subsection{Scope}

The scope of this thesis was to investigate organometallic compounds by advanced solution state NMR methods in order to build a bridge from known solid state structures to their behaviour in solution. Since these reagents are mostly applied in solution, knowledge of the structural motives could improve the possibility to tune their reactivity.

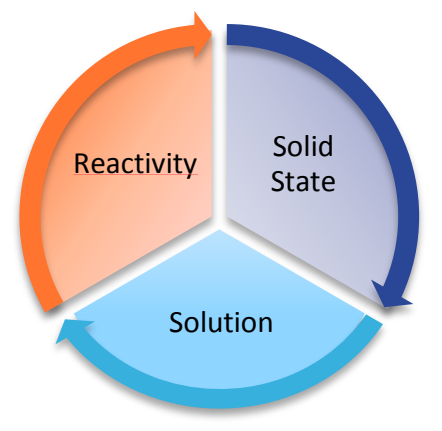

This work benefits from extensive knowledge about aggregation phenomena in the solid state which has already been acquired in the workgroup of D. Stalke. The projects presented in three separate chapters will be dedicated to NMR spectroscopy in isotropic solution, NMR in anisotropic environment and combined approaches.

On the basis of the crystal structure of ortho-lithiated $N, N$-dimethylaniline (DMA) $[t \mathrm{BuLi}]_{4} \cdot 4\left[\mathrm{Me}_{2} \mathrm{NC}_{6} \mathrm{H}_{4} \mathrm{Li}\right]_{4}$, which cocrystallizes with $1 / 4[t \mathrm{BuLi}]_{4}$ in the asymmetric unit, NMR spectroscopy should be employed to judge on the behaviour of this interesting compound in solution. The second part of the chapter on isotropic NMR experiments is centred around a detailed investigation of 2-thienyllithium. Crystal structures of differently aggregated species ranging from tetrameric to dimeric and monomeric arrangements, tuneable by addition of stoichiometric amounts of Lewis bases, were characterized prior to this work. Starting from firm ground by dissolving crystalline material, ${ }^{1} \mathrm{H},{ }^{7} \mathrm{Li}-\mathrm{HOESY}$ data along with other 2D NMR experiments should yield a broader understanding of the solution state structure obtained.
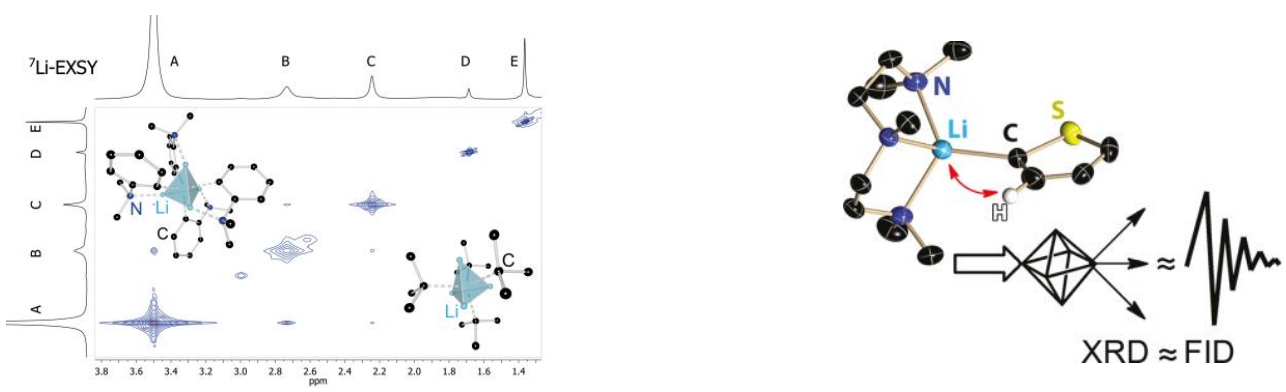

Figure 1.24: Illustration of the projects presented in the chapter on structure elucidation by isotropic NMR experiments. 
To overcome the necessity of solid state structure determination in advance of NMR spectroscopic measurements, the central part of this work will be concerned with the application of anisotropic NMR spectroscopy, generated by strain induced alignment in a (polymer) gel (SAG), to organometallic compounds. For that purpose, the obstacle of the high reactivity of this class of compounds should be considered along with measurements of anisotropic NMR parameters. Due to the quadrupolar nature of ${ }^{7} \mathrm{Li}$, residual quadrupolar couplings (RQCs) become an observable that represent a valuable source of information. Working with polymer gels inspired me to evaluate the limitations of SAG, to investigate the structure of the gel itself and to modify the polymer network.
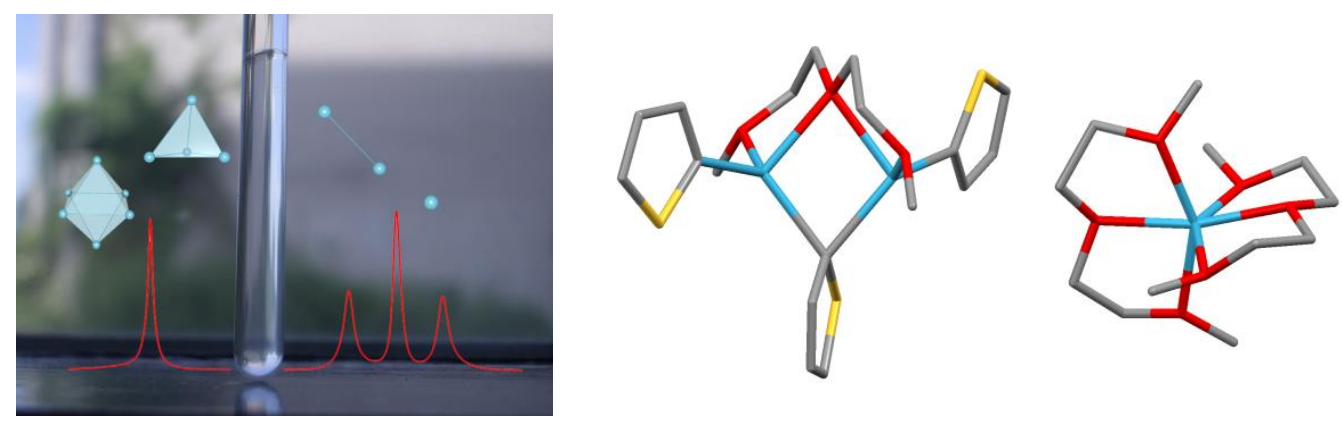

Figure 1.25: Illustration of the SAG method for lithiumorganic compounds (left) and optimized geometries for the crystal structure $\left[\mathrm{Li}(\text { diglyme })_{2}\right]\left[(\right.$ diglyme $\left.) \mathrm{Li}_{2}\left(\mathrm{C}_{4} \mathrm{H}_{3} \mathrm{~S}\right)_{3}\right]$ (right).

The last part focuses on the employment of complementary methods for structure evaluation in solution. A combined study of Grignard and Turbo-Grignard compounds is based on ESI mass spectrometry measurements which shall be accompanied by isotropic and anisotropic NMR investigations. The second part of this chapter on combined studies was initiated by the characterization of the interesting lithium lithiate crystal structure [Li(diglyme $\left.)_{2}\right]\left[(\right.$ diglyme $\left.) \mathrm{Li}_{2}\left(\mathrm{C}_{4} \mathrm{H}_{3} \mathrm{~S}\right)_{3}\right]$. To account for the behaviour of this complex in solution, isotropic and anisotropic NMR parameters as well as DFT calculations should be performed. 


\section{RESULTS AND DISCUSSION}

\subsection{Structure Elucidation by Isotropic NMR Experiments}

\subsubsection{Mixed Crystalline Lithiumorganics and Interconversion in Solution}

Due to their versatile application in synthetic chemistry, lithium organics ${ }^{[2 b, 2 c]}$ have been in the focus of various investigations, both in the solid state ${ }^{[6-7,25,27,115]}$ and in solution ${ }^{[43,16]}$. Moreover, the effect of directing groups for selective metallation of arenes has attracted attention over the last decades ranging from purely theoretical studies to combined studies including NMR and X-ray diffraction. ${ }^{[117]}$ A prominent subject of investigation is $\mathrm{N}, \mathrm{N}$ dimethylaniline (DMA), which is readily metalated in the ortho position by adding conventional $n \mathrm{BuLi}$ in hexane and refluxing for $12 \mathrm{~h},{ }^{[118]}$ and only recently, an orthoborylaniline derived from $o$-lithiated DMA was confirmed to display $\mathrm{H}_{2}$-activating properties at nearly ambient conditions. ${ }^{[119]}$ Mechanistic studies have, among others, been performed by Bauer and Schleyer yielding an understanding of the directing properties of these substituents by NMR spectroscopy and theoretical calculations. ${ }^{[120]}$ Characterizing the ortho-lithiated DMA unambiguously by X-ray diffraction and then starting from firm ground to study its behaviour in solution seemed to be a promising approach. In a previous work, DMA and $t \mathrm{BuLi}$ were mixed and the yellow filtrate was stored at room temperature for one day providing colourless needles suitable for X-ray analysis. ${ }^{[121]}$
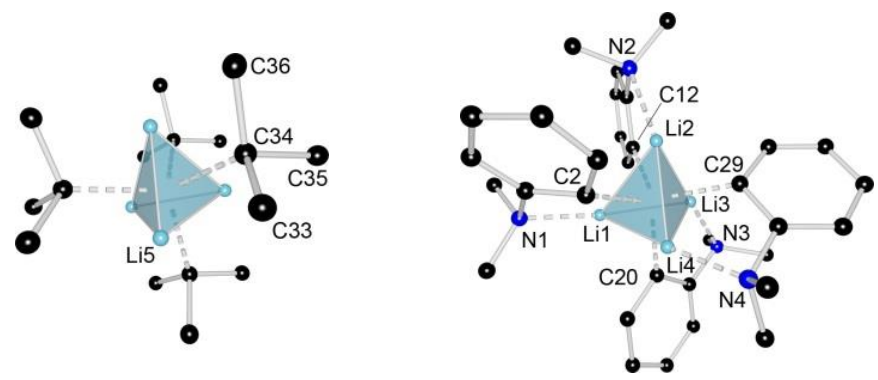

Figure 2.1: Crystal structure of $1[t \mathrm{BuLi}]_{4} \cdot 4\left[\mathrm{Me}_{2} \mathrm{NC}_{6} \mathrm{H}_{4} \mathrm{Li}\right]_{4} \cdot{ }^{[121]}$ The $\left[\mathrm{Me}_{2} \mathrm{NC}_{6} \mathrm{H}_{4} \mathrm{Li}\right]_{4}$ moiety occupies a general position while the $[t \mathrm{BuLi}]_{4}$ unit is only present at a quarter in the asymmetric unit. Hydrogen atoms are omitted for clarity.

Interestingly, the unit cell of $[t \mathrm{BuLi}]_{4} \cdot 4\left[\mathrm{Me}_{2} \mathrm{NC}_{6} \mathrm{H}_{4} \mathrm{Li}\right]_{4} \mathbf{1}$, contains two different lithium organic aggregates and thus represents a seldom case of cocrystallization in lithium organic chemistry (Figure 2.1). One is the $\left[\mathrm{Me}_{2} \mathrm{NC}_{6} \mathrm{H}_{4} \mathrm{Li}\right]_{4}$ tetramer, reminiscent of the $\mathrm{CH}_{2}$-bridged 
DMA analogue $N, N$-dimethylbenzylamine characterized by van Koten et al. ${ }^{[122]}$, the other is a $[t \mathrm{BuLi}]_{4}$ moiety which exhibits the same structural features as the donor-free parent material. ${ }^{[23]}$ To the best of my knowledge, only one other example of a cocrystallized lithium organic aggregate has been reported so far: Mitzel and co-workers could characterize 2-lithio-1,3-dimethyl-1,3-diazacyclohexane which crystallizes as a tetramer with one $1 / 4[t \mathrm{BuLi}]_{4}$ moiety of a tetrahedron in the asymmetric unit. ${ }^{[123]}$ The analogy to $\mathbf{1}$ is further underlined by the fact that both compounds crystallize in the tetragonal space group $I \overline{4}$. While cocrystallization is a rare phenomenon in lithiumorganic chemistry, cocrystallized solvent molecules are quite common. ${ }^{[124]}$ Moreover, the principle of forming cocrystals is frequently applied in pharmacological studies to fine-tune the properties of active pharmaceutical ingredients (APIs) by selection of a conformer or to design a crystallization process for manufacturing. ${ }^{[125]}$ The isolated structural motif itself - triangles formed by lithium cations which are $\mu_{3}$-capped by the carbanionic $\mathrm{C}_{\alpha}$-atom - is a leading building block in lithium organic chemistry. The so formed $\mathrm{Li}_{4}$-tetrahedron is found in a variety of lithium organic tetramers, e.g. $[\mathrm{EtLi}]_{4},{ }^{\left[{ }^{[}\right]}$ortho-lithiated methoxybenzene ${ }^{[126]}$ and the $\mathrm{Na} / \mathrm{Li}$ mixed metal species derived from sodium methyl(4-methylbenzyl)amide. ${ }^{[127]}$ In agreement with the presented solid state structure of $\mathbf{1}$, the ${ }^{7} \mathrm{Li}$ NMR spectrum should show two signals indicative of the two different homo-tetrameric species $\left[\mathrm{Me}_{2} \mathrm{NC}_{6} \mathrm{H}_{4} \mathrm{Li}\right]_{4}$ and $[t \mathrm{BuLi}]_{4}$. However, taking into account the fact that cocrystals show slightly varied properties compared to the pure compounds, for instance a different solubility, ${ }^{[128]}$ it is not totally unexpected that the resulting ${ }^{7} \mathrm{Li}$ NMR spectrum in toluene- $d_{8}$ displays five relatively sharp signals accompanied by several smaller and broader ones (Figure 2.2) instead of only two:

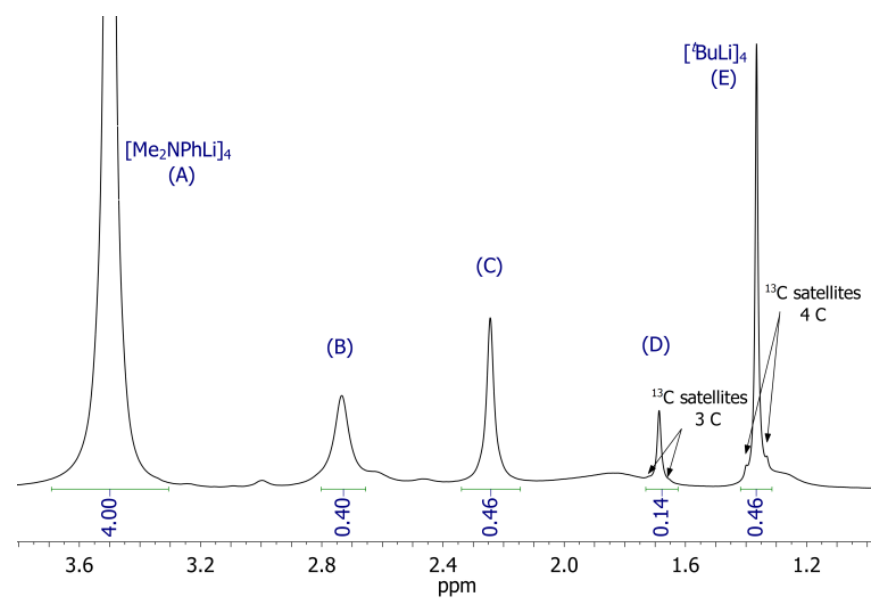

Figure 2.2: ${ }^{7} \mathrm{Li}$ NMR spectrum of 1 in toluene- $d_{8}(298 \mathrm{~K})$ including integrals and tentative assignments.

The main peak (A) with a chemical shift of $3.5 \mathrm{ppm}$ is tentatively assigned to the homotetramer $\left[\mathrm{Me}_{2} \mathrm{NC}_{6} \mathrm{H}_{4} \mathrm{Li}\right]_{4}$, while the peak at $1.4 \mathrm{ppm}(\mathbf{E})$ shows ${ }^{13} \mathrm{C}$ satellites with a 
coupling constant of $10.6 \mathrm{~Hz}$ which is in agreement with a fluxional $t \mathrm{BuLi}$ homotetramer. Lowering the temperature to $223 \mathrm{~K}$, results in a static tetrameric structure with a coupling constant of $14.5 \mathrm{~Hz}$. The corresponding ${ }^{13} \mathrm{C}$ NMR spectrum at $223 \mathrm{~K}$ shows ten lines as would be expected for a coupling to three $I=3 / 2$ nuclei. This assignment is confirmed with a pure sample of $t \mathrm{BuLi}$ in toluene- $d_{8} \cdot{ }^{13} \mathrm{C}$ satellites are also observed for the peak (D) at $1.7 \mathrm{ppm}$, where the integral of the satellites indicates three instead of four $t \mathrm{BuLi}$ units (Figure 2.3). Satellites are neither observed for DMA moieties nor for the broader peaks A, $\mathbf{B}$ and $\mathbf{C}$ in general. The effect that only sterically hindered and less dynamic compounds like $t \mathrm{BuLi}$ show ${ }^{13} \mathrm{C}$ satellites is well known in literature. ${ }^{[8 \mathrm{~b}, 39 \mathrm{~d}]}$

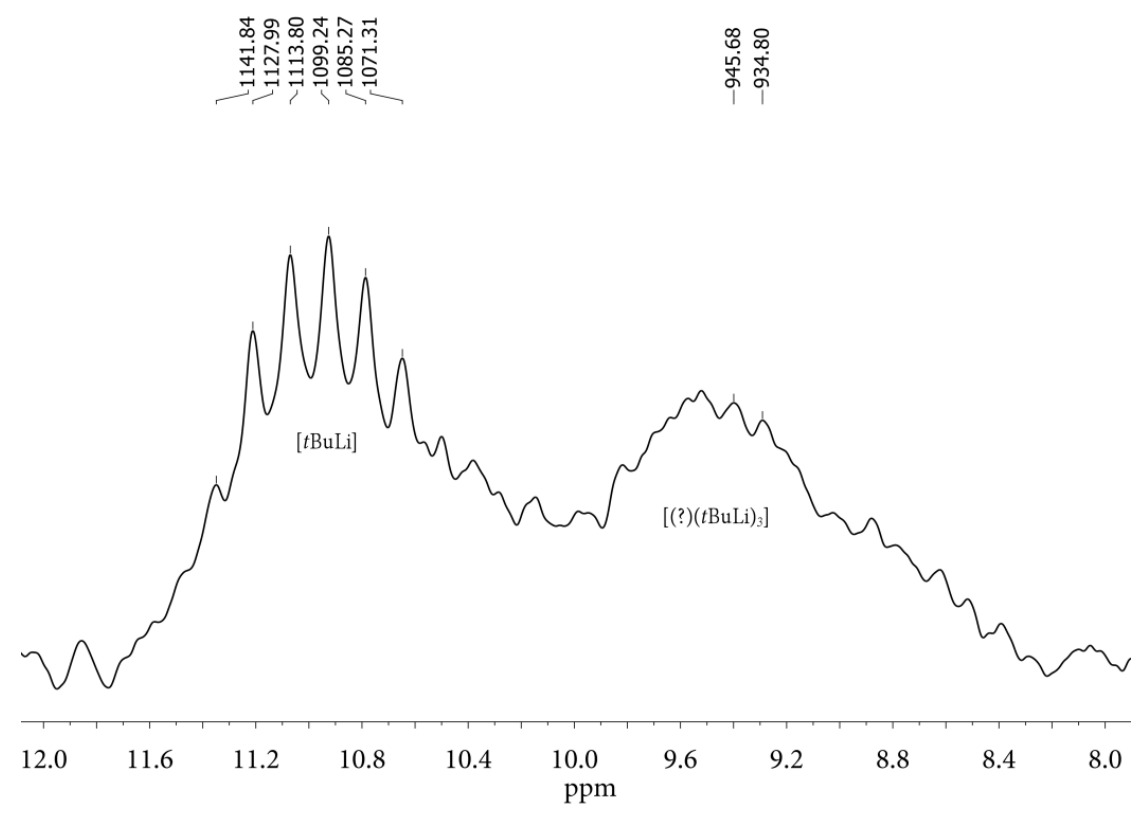

Figure 2.3: Extract from the ${ }^{13} \mathrm{C}$ NMR spectrum of 1 at $223 \mathrm{~K} .{ }^{1} \mathrm{~J}_{\mathrm{LiC}}$ couplings of $13.8 \mathrm{~Hz}$ can be extracted for $[t \mathrm{BuLi}]_{4}(\mathbf{E})$ while compounds $\mathbf{B}, \mathbf{C}$ and $\mathbf{D}$ give unresolved multiplets around $9 \mathrm{ppm}$.

Taking into account that ${ }^{1} \mathrm{H}$ as well as ${ }^{13} \mathrm{C}$ NMR spectra at room temperature also diplay multiple signal sets for the lithiated DMA and $t$ BuLi units and that two of them could be assigned as homotetramers, the formation of mixed aggregates seems to be the most conceivable explanation for the observed phenomena. Variable experiments were consulted to verify this assumption. Among those, ${ }^{7} \mathrm{Li}$ diffusion-ordered spectroscopy (DOSY) proved to be the most valuable and illustrative method as the monotonically decreasing diffusion coefficient $D$ (vertical axis, Figure 2.4) evidences the presence of mixed tetrameric aggregates, namely $\left[\mathrm{Me}_{2} \mathrm{NC}_{6} \mathrm{H}_{4} \mathrm{Li}\right]_{4}(\mathbf{A}),\left[\left(\mathrm{Me}_{2} \mathrm{NC}_{6} \mathrm{H}_{4} \mathrm{Li}\right)_{3}(t \mathrm{BuLi})\right]$ (B), $\left[\left(\mathrm{Me}_{2} \mathrm{NC}_{6} \mathrm{H}_{4} \mathrm{Li}\right)_{2}(t \mathrm{BuLi})_{2}\right](\mathbf{C}),\left[\left(\mathrm{Me}_{2} \mathrm{NC}_{6} \mathrm{H}_{4} \mathrm{Li}\right)(t \mathrm{BuLi})_{3}\right](\mathbf{D})$, and $[t \mathrm{BuLi}]_{4}(\mathbf{E})$. Consequently, each of the narrow peaks in the ${ }^{7} \mathrm{Li} \mathrm{NMR}$ spectrum is due to a consecutive substitution of one ortho-lithiumanilide by one $t \mathrm{BuLi}$ moiety in the tetrameric aggregate. The formation of such mixed aggregates has already been confirmed by X-ray crystallography for other, 
similar lithiumorganic compounds (e.g. $\mathrm{Li}_{4} \mathrm{R}_{2} n \mathrm{Bu}_{2}$ with $\mathrm{R}=2,6-\left(\mathrm{CH}_{2} \mathrm{NMe}_{2}\right)_{2}-3,5-$ $\left.\mathrm{Me}_{2} \mathrm{C}_{6} \mathrm{HCH}_{2}\right) \cdot{ }^{[129]}$ Besides, mixed aggregates are commonly established in mixtures of RLi and ROLi species, ${ }^{[130]}$ and investigations of propyllithium in solution by Fraenkel et al. further underline this postulation as they could prove the existence of five different aggregates in the non-donating solvent cyclopentane. ${ }^{[131]}$ A combination of mass spectrometry and NMR spectroscopy allowed van Koten et al. to shed light on the behaviour of tetranuclear arylcopper and arylithium species as well as their inter-aggregate exchange between identical (DMA) or closely related $\left(\mathrm{CH}_{2}\right.$ bridge between aromatic ring and dimethylamino group) aryl moieties. ${ }^{[132]}$ Furthermore, the DOSY spectrum of 1 hints at the possibility that the remaining broad signals from the initial ${ }^{7} \mathrm{Li}$ NMR spectrum (Figure 2.2) might belong to homo- or heterodimers, but broad signals are generally weak in DOSY spectra making the diffusion coefficients less reliable. Nevertheless, the existence of similar dimers has been extensively studied by Reich et al. who also could show that amine-chelated aryllithium reagents also display chelation isomers. ${ }^{[133]}$

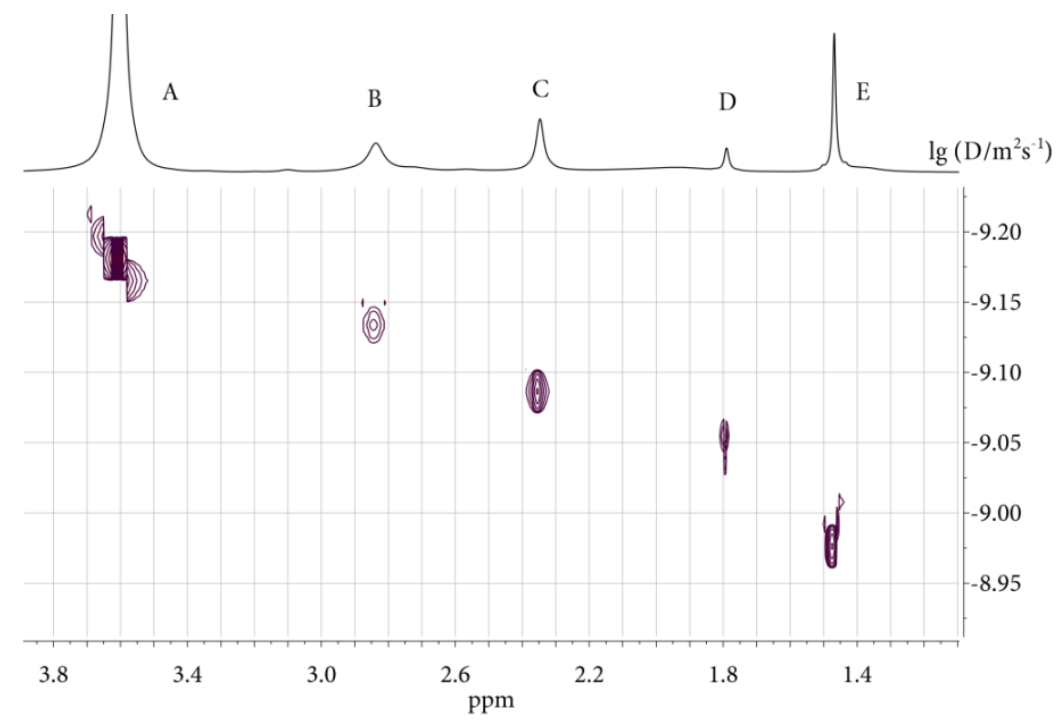

Figure 2.4: ${ }^{7} \mathrm{Li}-\mathrm{DOSY}$ spectrum of $[t \mathrm{BuLi}]_{4} \cdot 4\left[\mathrm{Me}_{2} \mathrm{NC}_{6} \mathrm{H}_{4} \mathrm{Li}\right]_{4}(\mathbf{1})$ in toluene- $d_{8}(298 \mathrm{~K})$.

The existence of mixed aggregates is also consistent with the ${ }^{7} \mathrm{Li}$ NMR spectra recorded at low temperature. For example, at $248 \mathrm{~K}$ broadening and splitting of the ${ }^{7} \mathrm{Li}$ signal at $2.3 \mathrm{ppm}(\mathrm{C})$ into two equally intense lines can be observed. Apparently, a deceleration of the otherwise fast intra-aggregate exchange in the two-by-two aggregate $\left[\left(\mathrm{Me}_{2} \mathrm{NC}_{6} \mathrm{H}_{4} \mathrm{Li}\right)_{2}(t \mathrm{BuLi})_{2}\right]$ results in two inequivalent lithium sites (Figure 2.5). Lowering the temperature further, signals that belong to mixed tetramers are broadened out completely presumably due to low-barrier processes like aryl rotations. The only two remaining signals $\mathbf{A}$ and $\mathbf{E}$ show an integral ratio of almost 4:1 as would be expected for the 
crystalline starting arrangement, which could also be verified by solid state ${ }^{13} \mathrm{C}$ NMR spectroscopy carried out by Hannes Faßhuber in the work group of Adam Lange.

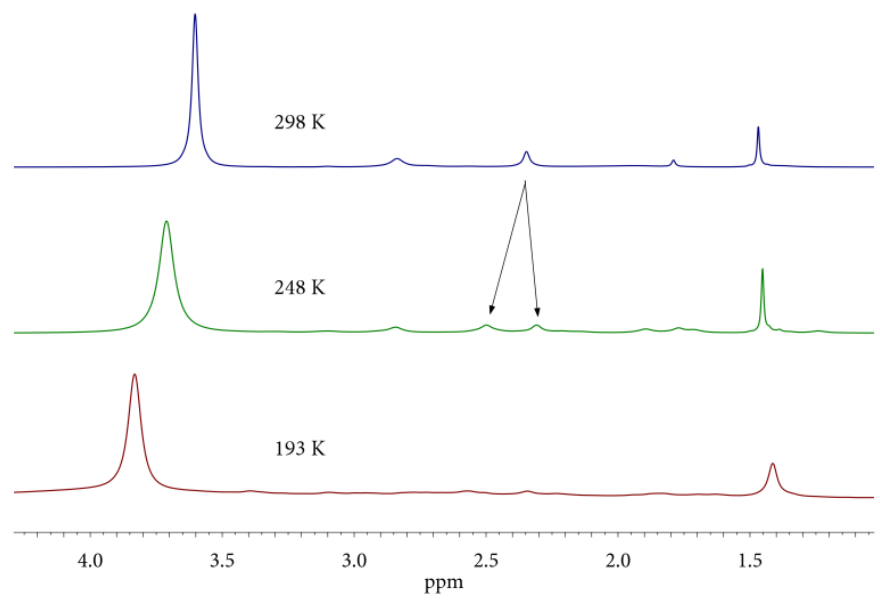

Figure 2.5: Stacked plot of the ${ }^{7} \mathrm{Li}$ NMR spectra of 1 in toluene- $d_{8}$ recorded at 193, 248, and $298 \mathrm{~K}$. The black arrows indicate splitting of peak $\mathrm{C}$ due to slowing down of the - at room temperature - fast intra-aggregate exchange.

To further assign the multiple signal sets that were also observed in the ${ }^{1} \mathrm{H}$ and ${ }^{13} \mathrm{C}$ NMR spectra of 1 a series of two-dimensional experiments was performed. First, ${ }^{1} \mathrm{H},{ }^{7} \mathrm{Li}-\mathrm{HOESY}$ experiments directly correlated the less crowded ${ }^{7} \mathrm{Li}$ NMR spectrum with the much more complex proton NMR spectrum via HOE interactions through space. The integral ratios derived from the corresponding signals in the ${ }^{1} \mathrm{H}$ and ${ }^{7} \mathrm{Li} \mathrm{NMR}$ spectra show good agreement, and so do diffusion coefficients determined by ${ }^{1} \mathrm{H}$ DOSY experiments. In a second step, standard ${ }^{1} \mathrm{H}, \mathrm{H}$-COSY (proximity through bonds) and -NOESY experiments (spatial proximity) consecutively allowed the assignment of the whole explicit constituents, and the combination of ${ }^{1} \mathrm{H},{ }^{13} \mathrm{C}$ correlation spectra (HSQC and $\mathrm{HMBC}$ ) enabled to draw conclusion about the corresponding ${ }^{13} \mathrm{C}$ chemical shifts of the different moieties.

Interestingly, performing ${ }^{7} \mathrm{Li}$-EXSY experiments on this system reveals that four of the five identified species are interconvertible giving rise to numerous cross-peaks in the spectrum (Figure 2.6). 


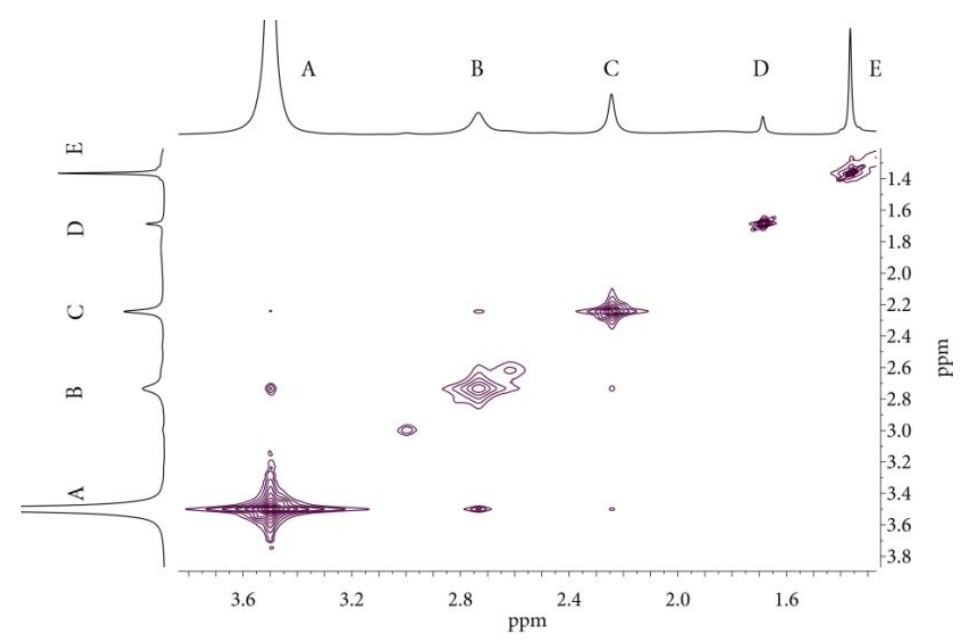

Figure 2.6: ${ }^{7} \mathrm{Li}-\mathrm{EXSY}$ NMR spectrum of (1) with a mixing time of $0.05 \mathrm{~s}$ at $298 \mathrm{~K}$.

The spectrum was recorded with a mixing time of $50 \mathrm{~ms}$ and a slow equilibration between the aggregates $\mathbf{A}-\mathbf{C}$ can be clearly observed while crosspeaks with aggregate $\mathbf{D}$ are very weak. Contrary to this, $[t \mathrm{BuLi}]_{4}$ does not take place in the inter-aggregate exchange within the observed mixing time. The existence of exchange cross-peaks not only between direct neighbours (A-B, B-C, and C-D) but also between the more remotely related species, might be evidence that simultaneous substitution of more than one moiety at once may also occur. However, the corresponding exchange rate constants $\left(\mathrm{s}^{-1}\right)$ determined from a saturated solution of 1 in toluene- $d_{8}$ (Scheme 2.1 ) do not give a definite answer. Using ${ }^{7} \mathrm{Li}$ EXSY integrals (mixing time: $50 \mathrm{~ms}$, reference: $5 \mathrm{~ms}$ ) the calculation of these rates was facilitated by the program EXSYcalc (MestreLab Research, Santiago de Compostela). Nevertheless, some exchange rates were too small to be determined precisely.

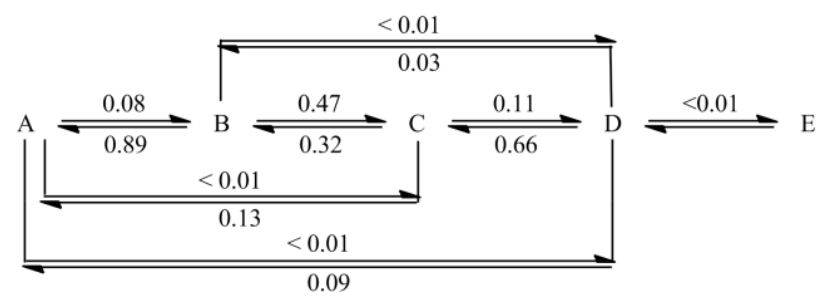

Scheme 2.1: Exchange rate constants in $\mathrm{s}^{-1}$ between the different tetramers in a saturated solution of 1 .

To gain more information about the dependencies of the postulated dynamics in solution, two reaction monitorings with 20 and $50 \mathrm{mg}$ DMA and the corresponding 1.25 equivalents of $t \mathrm{BuLi}$ were carried out inside the NMR tube over the range of two weeks. Thereby, it becomes apparent that both the lithiation reaction itself and the lithium exchange at equilibrium are significantly slower for the more diluted sample. This result disfavours an exchange mechanism implying a breaking up and re-formation of the aggregates, leaving collisions between tetrameric units to be the most probable exchange pathway. 
In summary, starting from the firm ground of the solid state structure of $\mathbf{1}$, it was not only possible to identify the five sharp signals from the ${ }^{7} \mathrm{Li}$ NMR spectrum to be homotetramers and mixed tetramers, but also to ascertain their mutual inconvertibility. Thereby, exchange rates could be analysed and the performed experiments emphasize the fact that the $a b$ initio plausible aggregates (A-E) are not statistically distributed. On the contrary, the mixed aggregated $\mathbf{B}-\mathbf{D}$ are rather disfavoured in comparison to the homotetramers $\mathbf{A}$ and $\mathbf{E}$ (no exchange to $\mathbf{E}$ on the NMR timescale, integrals etc.), which is consistent with the crystallized, thus - under the crystallization conditions - thermodynamically most stable structure in the solid state.

\subsubsection{Aggregation of Donor Base Stabilized 2-Thienyllithium in Solution}

The electron-rich, sulfur-containing heterocycle thiophene is a noteworthy motif in various chemical areas extending from scaffolds in drugs (medical science, anticancer treatment $)^{[134]}$ to functionalized poly-, ${ }^{[135]}$ oligo- $^{[136]}$ or fused thiophenes ${ }^{[137]}$ and transition metal complexes ${ }^{[138]}$ in material science. To introduce the thienyl moiety into more complex structures, the employment of metalated species is by far the most straightforward method available. ${ }^{[139]}$ Either by halogen-lithium exchange ${ }^{[140]}$ or by deprotonation at the most acidic position, ${ }^{[141]}$ lithiated thiophenes are readily accessible. With a pKa value of 33 , the 2-position surpasses the 3-position by $\sim 5$ units. ${ }^{[142]}$ Thus, a metalation in 3-position is only feasible by halogen-lithium exchange. However, this reaction is temperaturedependent and leads to a halogen-transfer-based so called "halogen dance process" at $0{ }^{\circ} \mathrm{C}$ or room temperature yielding a mixture of differently substituted derivatives. ${ }^{[143]}$ Moreover, an excess of the applied reagent may also favour the formation of 2,5-dilithated species. ${ }^{[144]}$ In order to employ lithiated thiophenes, it is vital to get information about the solid state structure and, even more important, their structure and behaviour in solution. Structural changes in solution like aggregation and possible solvation determine the reactivity and selectivity in syntheses and thereby the material and its characteristics.

Of thienyllithium, several single-crystal X-ray structures have been either published or deposited in the Cambridge Structural Database. ${ }^{[145]}$ Herein, only the tmeda-coordinated dimer $\left[(\mathrm{TMEDA}) \mathrm{Li}\left(\mathrm{C}_{4} \mathrm{H}_{3} \mathrm{~S}\right)\right]_{2}$ from now on referred to as $\mathbf{2 d}$ is emphasized. Speck and Smeets measured this crystal structure in 1999 and deposited it in the Cambridge Structural Database. ${ }^{[146]}$ From this structure the average $\mathrm{Li}-\mathrm{C}_{\alpha}$ distance was extracted and is discussed later on in this chapter. With respect to analysis in solution, thienyllithiums have caught the attention of NMR spectroscopists for many years. Starting in 1968, Gronowitz and Bugge reported the proton chemical shifts and coupling constants and featured that both 
parameters are affected in a way opposite to vinyl compounds. ${ }^{[147]}$ Already at this stage they pointed out 2-thienyllithium as an interesting object of study. This was further supported by the detection of unusual coupling constants, e.g. a very small ${ }^{1} J_{\mathrm{C} 2 \mathrm{C} 3}$ coupling of $30 \mathrm{~Hz}$ and a very large and positive ${ }^{2} J_{\mathrm{C} 2 \mathrm{H} 3}$ coupling of $20 \mathrm{~Hz} \cdot{ }^{[148]}$ This is reliable data and interesting even more, because the carbon-carbon couplings across one bond depend only insignificantly on the state of aggregation. ${ }^{[149]}$ Interestingly, thienyllithium has also been extensively studied with respect to its cation stabilization properties. Therein, chemical shift differences of thienyl-homologues of the trityl cation upon ionization have been recorded and compared to calculated $\pi$ charge densities. ${ }^{[150]}$ Finally, Reich and co-workers investigated various substituted 2-lithiated thiophenes in order to address the phenomenon of aggregation and chelation in solution. ${ }^{[16 b]}$

Our group started to thoroughly characterize unsubstituted 2-thienyllithium and its aggregation. Therefore, thiophene was reacted with $n \mathrm{BuLi}$ at $0^{\circ} \mathrm{C}$ in a $1: 1$ stoichiometric ratio in diethyl ether followed by subsequent addition of the corresponding donor bases (THF, DME, TMEDA and PMDETA). Crystals suitable for X-ray diffraction experiments were obtained by low-temperature crystallization (Figure 2.7). ${ }^{[10]}$

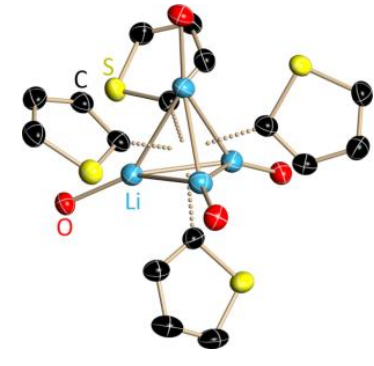

$2 \mathbf{a}$

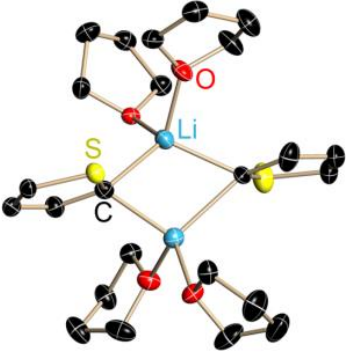

$2 \mathbf{b}$

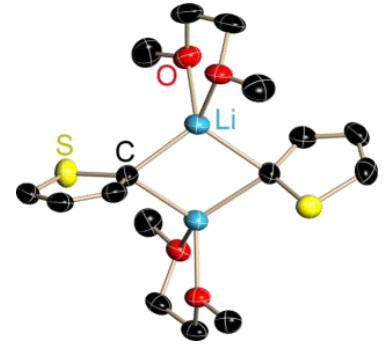

$2 \mathrm{c}$

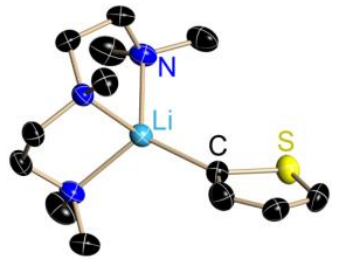

$2 \mathrm{e}$

Figure 2.7: Molecular structures of $\left[\left(\mathrm{Et}_{2} \mathrm{O}\right) \mathrm{Li}_{(}\left(\mathrm{C}_{4} \mathrm{H}_{3} \mathrm{~S}\right)\right]_{4}(\mathbf{2 a}),\left[(\mathrm{THF})_{2} \mathrm{Li}_{(}\left(\mathrm{C}_{4} \mathrm{H}_{3} \mathrm{~S}\right)\right]_{2}(\mathbf{2 b}),\left[(\mathrm{DME}) \mathrm{Li}\left(\mathrm{C}_{4} \mathrm{H}_{3} \mathrm{~S}\right)\right]_{2}(\mathbf{2 c})$ and [(PMDETA) $\left.\mathrm{Li}\left(\mathrm{C}_{4} \mathrm{H}_{3} \mathrm{~S}\right)\right](\mathbf{2 e})$. Hydrogen atoms and oxygen-bound ethyl groups are omitted for clarity. The depicted crystal structures were measured and refined by Markus Granitzka. ${ }^{[151]}$

Similar to other organolithium deaggregation series, the tetramer with diethyl ether coordination is disaggregated by addition of THF, DME and TMEDA resulting in a dimeric structural motif with a $\mathrm{Li}_{2} \mathrm{C}_{2}$-core $(\mathbf{2} \mathbf{b}$-d) in the solid state. The synthesis of the previously known dimer $\left[(\mathrm{TMEDA}) \mathrm{Li}\left(\mathrm{C}_{4} \mathrm{H}_{3} \mathrm{~S}\right)\right]_{2} \mathbf{2 d}$ was repeated by Markus Granitzka and confirmed by X-ray diffraction yielding similar data to that measured by Speck and Smeets. ${ }^{[146]}$ The three-dentate $\mathrm{N}$-donor PMDETA though, disaggregates 2-thienyllithium even further resulting in a monomeric coordination pattern. Interestingly, the extracted average $\mathrm{Li}-\mathrm{C}_{\alpha}$ distances show a clear trend, namely a shortening of the $\mathrm{Li}-\mathrm{C}_{\alpha}$ bonds from the tetramer via the dimers to the monomeric structure. Although it is still controversally discussed, whether these Li-C contacts are to be considered as mainly ionic or covalent, 
experimental charge density investigations recently performed in our group, support a predominantly ionic interaction which might be substantially affected by the presence of solvent molecules. ${ }^{[30]}$ Consequently, further studies of this deaggregation series in solution are of particular importance. For this purpose, the obtained crystals were dissolved in the non-donating solvent toluene- $d_{8}$. On the one hand this should guarantee a confident starting point, i.e. the solid state structure, and on the other hand it supplies a solvent environment that does not interfere with the coordination pattern found in the crystal structure.

Table 2.1: Average $\mathrm{Li}-\mathrm{C}_{\alpha}$ distances $(\AA)$ in the solid-state structures of 2-thienyllithium and ${ }^{13} \mathrm{C}$ chemical shifts in ppm of $\mathbf{2 a - e .}$

\begin{tabular}{ccc}
\hline & av. $\mathrm{Li}-\mathrm{C}_{\alpha}[\AA]$ & $\delta\left({ }^{13} \mathrm{C}\right)$ of $\mathrm{C}_{\alpha}[\mathrm{ppm}]$ \\
\hline $\mathbf{2 a}$ & $2.282(4)$ & 166.9 \\
$\mathbf{2 b}$ & $2.228(4)$ & 170.9 \\
$\mathbf{2 c}$ & $2.212(14)$ & 172.1 \\
$\mathbf{2 d}$ & $2.187^{[146]}$ & 174.4 \\
$\mathbf{2 e}$ & $2.115(3)$ & 180.1 \\
\hline
\end{tabular}

Unfortunately, crystals of the compounds $\mathbf{2 a}$ and $\mathbf{2 d}$ had to be dissolved in the presence of a small excess of the corresponding donor base due to their overall low solubility. For a general characterization of the five aggregates, standard one- and two-dimensional NMR techniques were applied. Whilst the ${ }^{1} \mathrm{H}$ and ${ }^{7} \mathrm{Li}$ chemical shifts of the compounds $2 \mathrm{a}-\mathbf{e}$ do not show a clear trend, the ${ }^{13} \mathrm{C}$ chemical shifts show an intriguing correlation with the lithium-carbon distances derived from the crystal structures (Table 2.1), hence, the shorter the $\mathrm{Li}-\mathrm{C}_{\alpha}$ distance, the higher the observed ${ }^{13} \mathrm{C}$ chemical shift value. Remarkably, even the small distance alterations for the three dimers are equally mirrored by a slight shift in the ${ }^{13} \mathrm{C}$ NMR spectrum (2b to $\mathbf{2 d}$ ). The carbon chemical shift is dominated by the paramagnetic shielding term ${ }^{[152]}$ which is considered to be responsible for the highfrequency shift. ${ }^{[40]}$ In our series, an increasing supply of electron density from the donating moieties (THF, DME, TMEDA, PMDETA) causes the electron-deficient $\mathrm{Li}^{+}$atom to demand less electron density from the $\mathrm{C}_{\alpha}$-carbon atom thus leaving this atom electronrich. The ${ }^{13} \mathrm{C}$ chemical shift as indicator for the aggregation behaviour in solution has been used for similar systems before. Reich et al. observed downfield shifts of $7.3 \mathrm{ppm}$ for 2thienyllithium and $8.2 \mathrm{ppm}$, for 3-methyl-2-tienyllithium, respectively, upon going from dimeric to monomeric arrangements, which is in good agreement with our numbers. ${ }^{[16 b]}$ For phenyllithium as a disaggregated compound, a wider range of ${ }^{13} \mathrm{C}$ chemical shifts from $196 \mathrm{ppm}$ for the $\mathrm{C}_{\alpha}$-carbon atom of the monomer via $188 \mathrm{ppm}$ (dimer) to a value of 
175 ppm (tetramer) has been described by different authors. ${ }^{[43,133 b, 153]}$ However, since chemical shifts also dependent on "external factors", e.g. temperature and solvent, the ${ }^{13} \mathrm{C}$ chemical shift of the $\mathrm{C}_{\alpha}$-carbon atoms represents a straightforward indicator but should be used with caution.
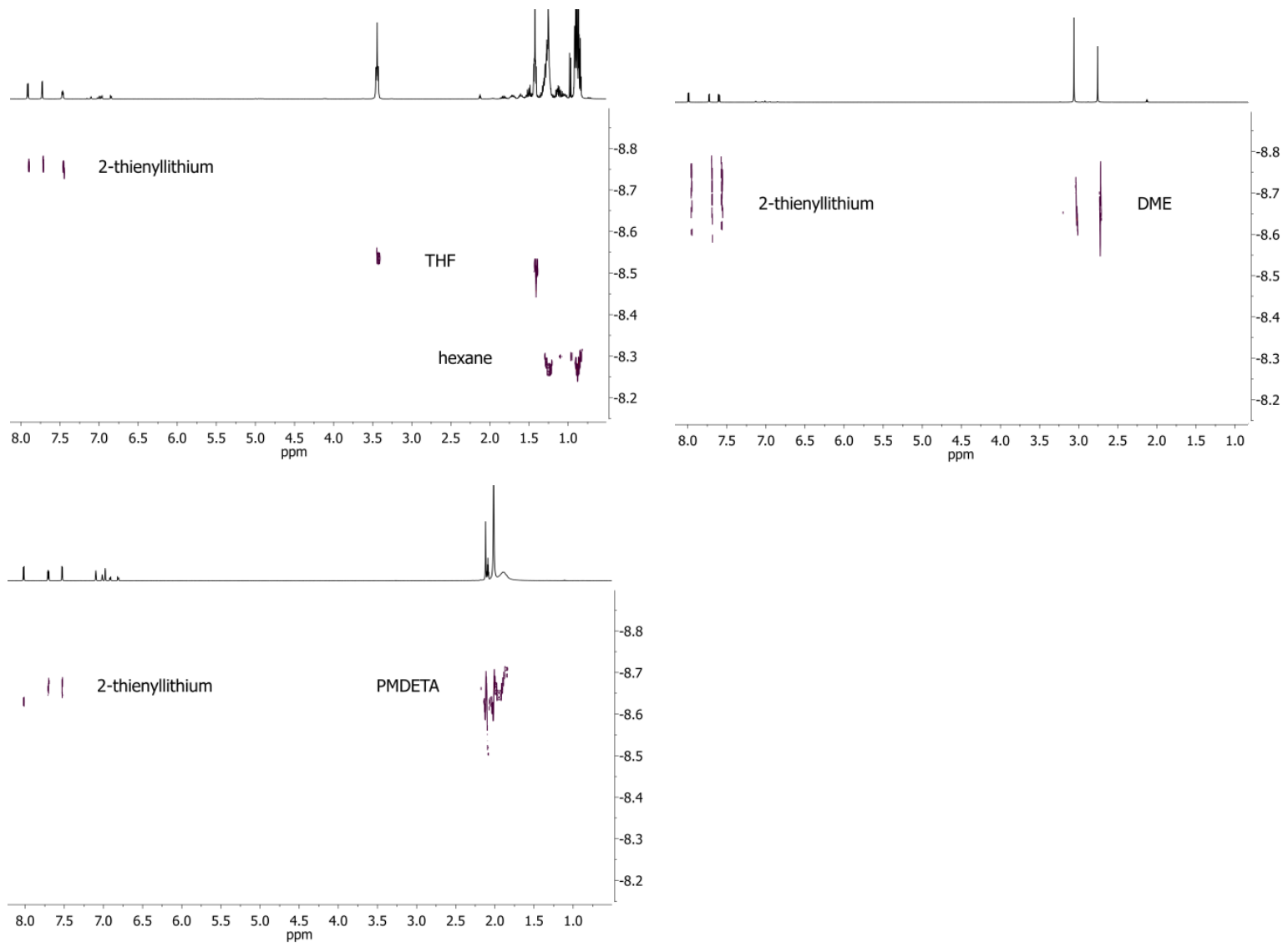

Figure 2.8: ${ }^{1} \mathrm{H}$-DOSY spectra of $\left[(\mathrm{THF})_{2} \mathrm{Li}\left(\mathrm{C}_{4} \mathrm{H}_{3} \mathrm{~S}\right)\right]_{2}$ (2b) (top left), $\left[(\mathrm{DME}) \mathrm{Li}\left(\mathrm{C}_{4} \mathrm{H}_{3} \mathrm{~S}\right)\right]_{2}$ (2c) (top right) and [(PMDETA) $\left.\mathrm{Li}\left(\mathrm{C}_{4} \mathrm{H}_{3} \mathrm{~S}\right)\right](\mathbf{2 e})$ (bottom left) in toluene- $d_{8}(298 \mathrm{~K})$. In the DOSY spectrum the horizontal axis shows the ${ }^{1} \mathrm{H}$ NMR spectrum whereas the diffusion coefficient in $\log D\left[\mathrm{~m}^{2} / \mathrm{s}\right]$ units is plotted on the vertical axis.

The solutions 2a-e were further investigated by means of DOSY experiments. On the one hand, this should verify that 2-thienyllithium and the corresponding Lewis base form one associated unit and on the other hand DOSY experiments are very versatile for the determination of the molecular size as described in the previous chapter. The ${ }^{1} \mathrm{H}$-DOSY spectrum of $\left[(\mathrm{DME}) \mathrm{Li}\left(\mathrm{C}_{4} \mathrm{H}_{3} \mathrm{~S}\right)\right]_{2}(2 \mathrm{c})$ in toluene- $d_{8}$ clearly demonstrates that the thienyl group and the donor moiety DME form a stable complex as both units display the same diffusion coefficient $\left(D=2,17 \cdot 10^{-9} \mathrm{~m}^{2} / \mathrm{s}\right)$ (Figure 2.8). The same holds true for the PMDETA coordinated structure $2 \mathrm{e}$ for which a diffusion coefficient of $\mathrm{D}=2.58 \cdot 10^{-9} \mathrm{~m}^{2} / \mathrm{s}$ could be extracted. Furthermore, the relative values of $D$ nicely fit to the aggregate size, as for smaller aggregates a faster translational motion and thus a bigger value for the diffusion coefficient is expected. For the less soluble compounds $\mathbf{2 a}$ and $\mathbf{2 d}$ the interpretation of the 
spectrum is hampered by the excess of donor base resulting in a smaller diffusion coefficient for the donor base compared to the thienyl group. On the timescale of the diffusion delay the coordinated molecules exchange with those free in solution and thus show an average value for the diffusion coefficient. For a solution of $\left[(\mathrm{THF})_{2} \mathrm{Li}_{(}\left(\mathrm{C}_{4} \mathrm{H}_{3} \mathrm{~S}\right)\right]_{2}$ (2b) in toluene- $d_{8}$ the ${ }^{1} \mathrm{H}$ NMR spectrum clearly confirms that no significant excess of THF is present. Nevertheless, the diffusion coefficient of THF $\left(D=3.08 \cdot 10^{-9} \mathrm{~m}^{2} / \mathrm{s}\right)$ varies significantly from the value obtained for the thienyl moiety $\left(D=1.85 \cdot 10^{-9} \mathrm{~m}^{2} / \mathrm{s}\right)$ although the value is still substantially lower than that of non-coordinating small molecules like toluene $\left(D=4.5 \cdot 10^{-9} \mathrm{~m}^{2} / \mathrm{s}\right)$ or hexane $\left(D=5.4 \cdot 10^{-9} \mathrm{~m}^{2} / \mathrm{s}\right)$ (Figure 2.8). This indicates that, in solution, THF molecules partially dissociate from the lithiated thiophene structure, possible leaving lithium atoms in a 3-fold-coordination. In principle, a higher aggregation e.g. in an equilibrium would also set free THF molecules. However, this could not be finally proven by the low temperature $(193 \mathrm{~K})$ reached in the corresponding experiments and would be in disagreement with the chemical shifts of Table 2.1.

A plot of the measured diffusion coefficients versus the molecular weight should yield a $\mathrm{D} \sim \mathrm{MW}^{-1 / 3}$ dependency. Although this trend is indicated for the series of molecules investigated herein (Figure 2.9), the low solubility of $\mathbf{2 a}$ and $\mathbf{2 d}$ impedes the exact analysis of the diffusion coefficients as the viscosity is significantly affected by the added co-solvents. The irregular behaviour observed in the case of the THF-coordinated dimer may also complicate the interpretation and comparison of $D$, and finally the correlation

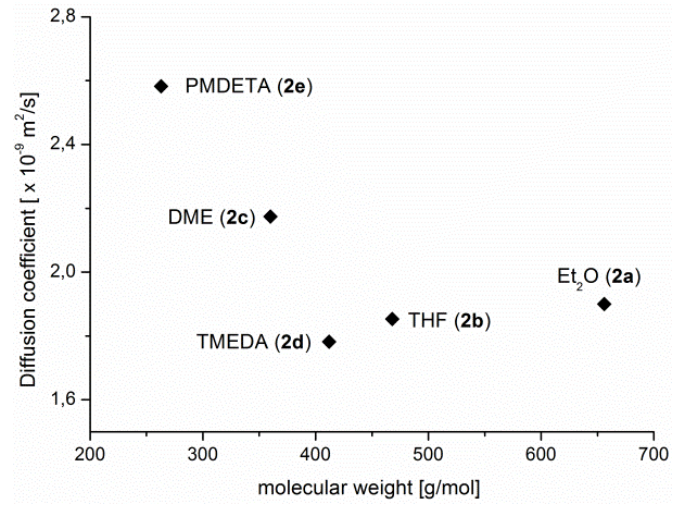

Figure 2.9: Plot of the diffusion coefficient of the thienyl resonances in $\mathbf{2 a - e}$, extracted from the corresponding DOSY experiments, versus the molecular weight. with the molecular weight is strictly only valid for ideal, rigid, spherical particles.

Another interesting particularity can be seen in the ${ }^{1} \mathrm{H}$-DOSY spectra of [(TMEDA) Li $\left.\left(\mathrm{C}_{4} \mathrm{H}_{3} \mathrm{~S}\right)\right]_{2}$ (2d) and [(PMDETA) $\left.\mathrm{Li}\left(\mathrm{C}_{4} \mathrm{H}_{3} \mathrm{~S}\right)\right]$ (2e): The apparent diffusion coefficient of the thiophene $\mathrm{H}-5$ signal (most downfield signal) is slightly larger than that of the protons $\mathrm{H}-3$ and $\mathrm{H}-4$ (Figure 2.9) although these protons belong to one and same molecular fragment. A closer inspection revealed that this signal slowly decreases with time (6\% during one ${ }^{1} \mathrm{H}$-DOSY experiment of $1 \mathrm{~h}$ ). At the same time, an increase of the proton signal assigned to residual toluene- $d_{7}$ (proton at the methyl group) is observed in contrast to the aromatic protons of toluene when preparing three samples with different 
concentrations of $2 \mathrm{e}$ (Figure 2.10, left). This could be explained by exchange of the $\mathrm{H}_{5}$ hydrogen atom of thienyllithium with deuterium atoms from toluene- $d_{8}$. To investigate the existence of a conceivable exchange process, a ${ }^{1} \mathrm{H}$-EXSY spectrum of $2 \mathrm{e}$ was recorded (Figure 2.10, right). Therein exchange peaks between 2-thienyllithium and free dissolved thiophene can be monitored.
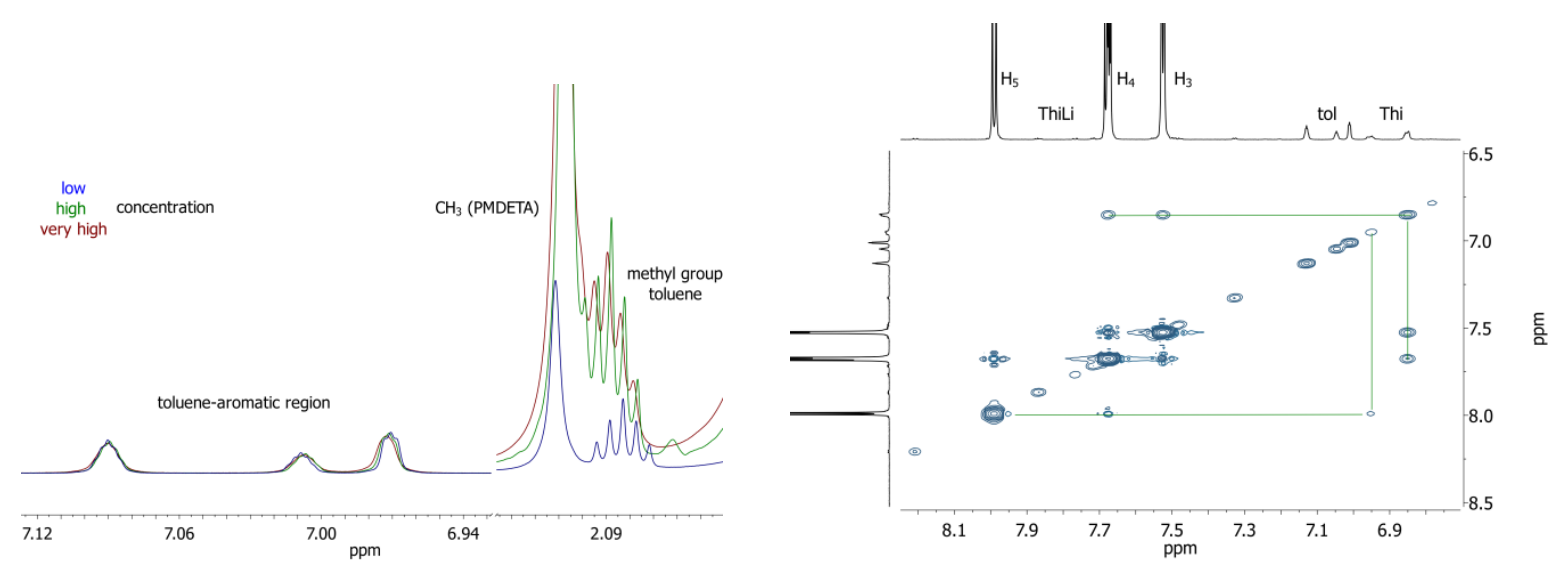

Figure 2.10: ${ }^{1} \mathrm{H}$ NMR spectra of solutions containing different concentrations of $2 \mathrm{e}$. The aromatic protons of toluene are superimposed and a different intensity for the methyl group due to protonation becomes obvious (left). Aromatic region from the ${ }^{1} \mathrm{H}$-EXSY spectrum of $2 \mathbf{e}$ in toluene- $d_{8}$ with residual thiophene (right).

As the 2,5-protons in thiophene are substantially more acidic compared to the methyl protons of toluene and no intermediate, lithiated toluene species can be detected by ${ }^{1} \mathrm{H}$ NMR spectroscopy, a H/D exchange via temporary lithiation of the methyl group in toluene as pictured in Scheme 2.2 seems reasonable. Interestingly, this process cannot be observed for solutions of $\mathbf{2 a - c}$ in toluene- $d_{8}$ which might account for the assumption that only the nitrogen-containing donor bases sufficiently increase the thienyl basicity to deprotonate toluene at room temperature. This is supported by Screttas and Eastham who promoted the deprotonation of toluene by $n \mathrm{BuLi}$ by adding tertiary amines. ${ }^{[154]}$

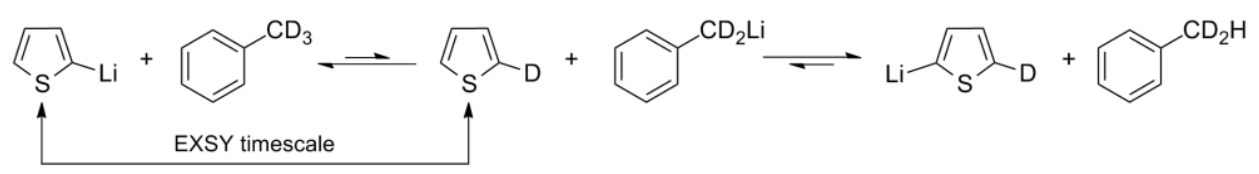

Scheme 2.2: Exchange process triggered by nitrogen-containing donor bases via temporary lithiation of toluene- $d_{8}$.

Since DOSY experiments did not provide a satisfactory answer to the behaviour of the 2thienyllithium series in solution the aggregation of $\mathbf{2 a - e}$ was alternatively studied using the ${ }^{1} \mathrm{H},{ }^{7} \mathrm{Li}$ heteronuclear Overhauser effect (HOE). All molecules contain several short protonlithium distances (up to $4 \AA$ ) in the solid state potentially yielding cross-peaks between the lithium atoms and the thienyl- and/or corresponding donor base fragments. Figure 2.11 depicts the ${ }^{1} \mathrm{H},{ }^{7} \mathrm{Li}-\mathrm{HOESY}$ spectra of $\mathbf{2 c}$ (left) and $\mathbf{2 e}$ (right) in toluene- $d_{8}$ as examples. For 
the DME-coordinated species, several cross-peaks can be nicely distinguished from the spectrum. The cross-peak at around $7.5 \mathrm{ppm}$ arises due to close proximity of a lithium atom and its neighbouring $\mathrm{H}_{3}$ proton of the thienyl moiety, whereas the two peaks between 2.5 and $3.0 \mathrm{ppm}$ are caused by the tightly bond donor base DME. In case of the spectrum of solution $2 \mathrm{e}$ (right) the same holds true for the cross-peaks with the donor base. The spectra of the remaining compounds exhibit a similar behaviour.
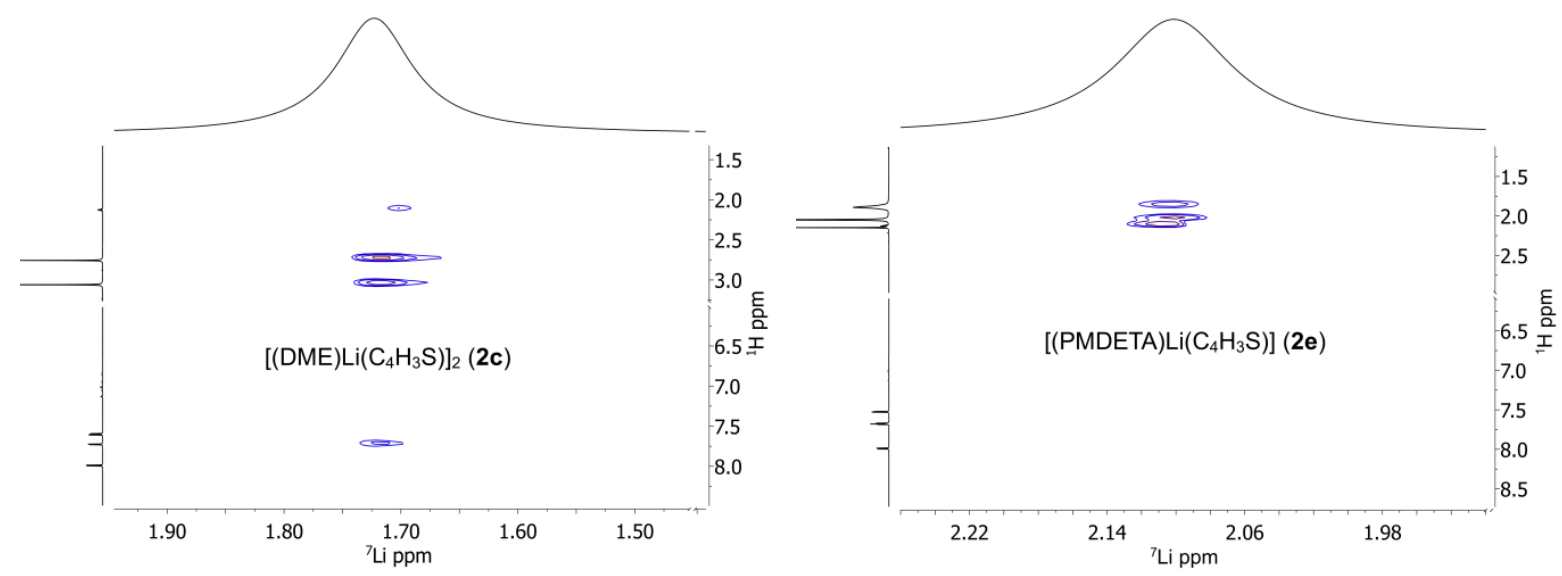

Figure 2.11: ${ }^{1} \mathrm{H},{ }^{7} \mathrm{Li}-\mathrm{HOESY}$ spectra of $\left[(\mathrm{DME}) \mathrm{Li}\left(\mathrm{C}_{4} \mathrm{H}_{3} \mathrm{~S}\right)\right]_{2}(\mathbf{2 c})$ (left) and $\left[(\mathrm{PMDETA}) \mathrm{Li}\left(\mathrm{C}_{4} \mathrm{H}_{3} \mathrm{~S}\right)\right]$ (2e) (right) recorded with a mixing time of $0.5 \mathrm{~s}$. The smaller signal at $2.0 \mathrm{ppm}$ is not a valid cross-peak, easily notable due to a phase difference. This is an artefact of the solvent toluene- $d_{8}$.

By varying the mixing time from 0.01 to $1.0 \mathrm{~s}$ characteristic build-up curves were recorded. All curves feature an initial linear increase of the cross peak intensity, a maximum after approximately $0.5 \mathrm{~s}$ followed by a decay of the intensity at higher mixing times due to longitudinal relaxation of the ${ }^{1} \mathrm{H}$ spins within the mixing time of the recorded spectra (Figure 2.12). ${ }^{[155]}$ Assuming a linear relationship between the slope of the initial build-up and the inverse sixth power of the proton-lithium distance, ${ }^{[156]}$ comparison of that slope with the $\Sigma r(\mathrm{H} \cdots \mathrm{Li})^{-6}$ term calculated from the crystal structures offers a handle to judge on whether the structures are retained in solution. Due to the low solubility of $\mathbf{2 a}$, cross peaks could be observed but they were not sufficiently strong for really short mixing times, preventing them to be included in this comparison. 

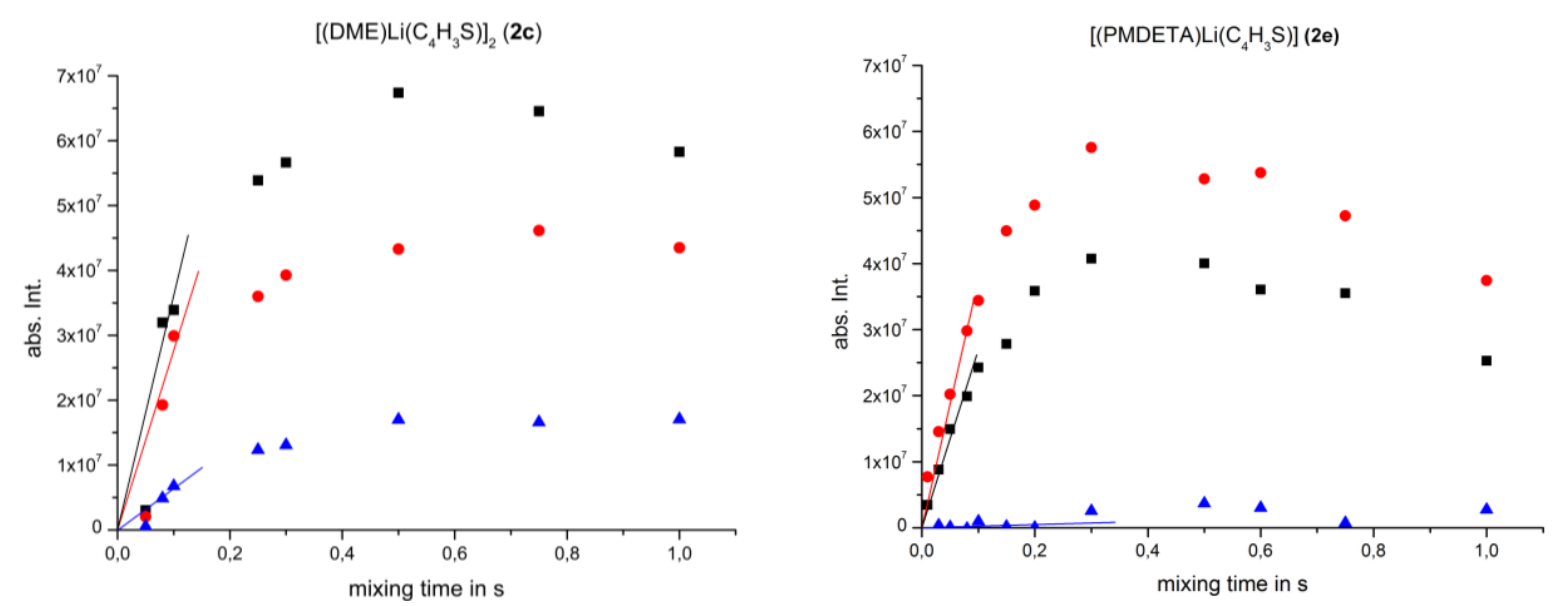

Figure 2.12: ${ }^{1} \mathrm{H},{ }^{7} \mathrm{Li}-\mathrm{HOESY}$ build-up curve of $\left[(\mathrm{DME}) \mathrm{Li}\left(\mathrm{C}_{4} \mathrm{H}_{3} \mathrm{~S}\right)\right]_{2}(\mathbf{2 c})$ and $\left[\left(\mathrm{PMDETA} \mathrm{Li}\left(\mathrm{C}_{4} \mathrm{H}_{3} \mathrm{~S}\right)\right](\mathbf{2 e})\right.$ in toluene- $d_{8}$. Mixing times were varied between 0.01 and $1.0 \mathrm{~s}$. Cross peaks could be observed for the neighbouring thiophene proton $\mathrm{H}_{3}(\bullet)$ and the $\mathrm{CH}_{2}-(\bullet) / \mathrm{CH}_{3}$-groups (•) of DME and PMDETA, respectively.

For the evaluation of the slope of the initial HOE build-up the data points of the shortest mixing times (0-0.1 s) were linear-fitted with the program ORIGIN. A broader range (up to 0.15 or $0.2 \mathrm{~s}$ ) led to a much higher error values for the linear fit and was therefore discarded. From the crystal structures, the lithium-proton distances were extracted for each lithium atom. Disorder in the crystal structure was also taken into account by averaging the corresponding distances. For instance, a $70 \%$ / $30 \%$ disorder of the thiophene molecule (rotation of $180^{\circ}$ ) was treated in the way that the distances of one fragment were multiplied by 0.7 and the distances derived for the other fragment with 0.3 , respectively. Finally, all distances were summed up according to $\Sigma r(\mathrm{H} \cdots \mathrm{Li})^{-6}$ and the ${ }^{1} \mathrm{H},{ }^{7} \mathrm{Li}-\mathrm{HOE}$ buildup was plotted against this sum (Figure 2.13).
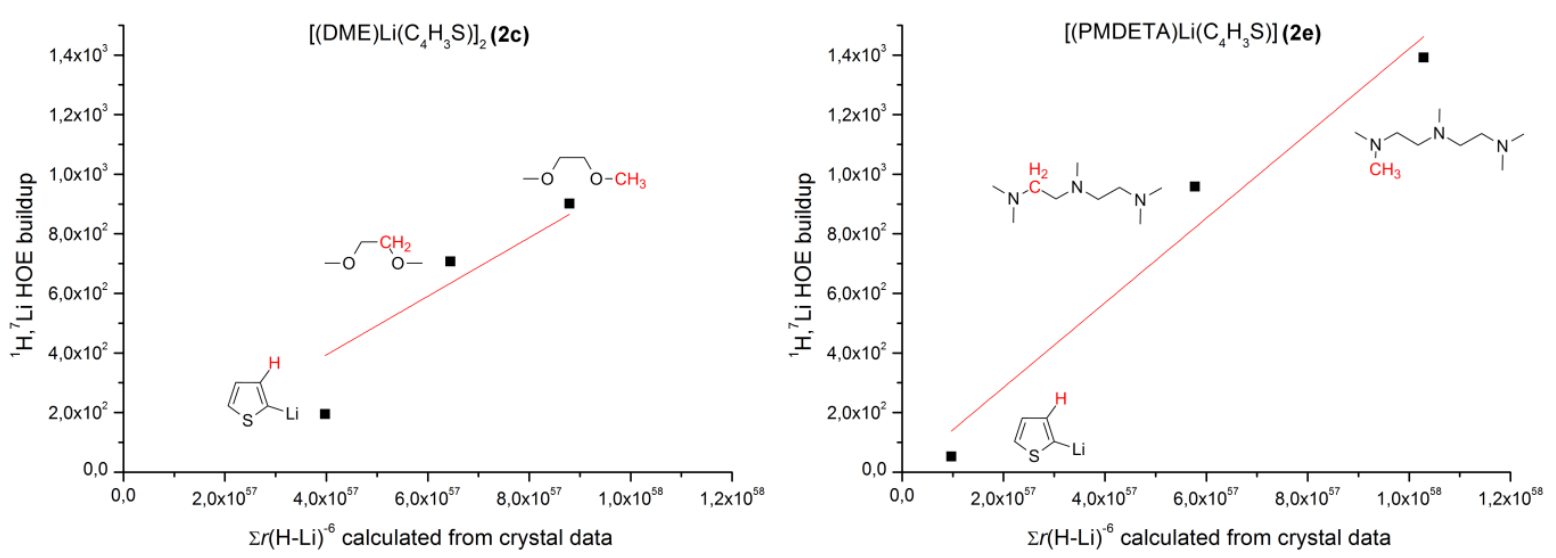

Figure 2.13: Comparison of the ${ }^{1} \mathrm{H},{ }^{7} \mathrm{Li}-\mathrm{HOE}$ build-up with the calculated $\Sigma r(\mathrm{H} \cdots \mathrm{Li})^{-6}$ terms from crystal data of $2 \mathrm{c}$ and $2 \mathrm{e}$. Disorder in the crystal structure has been taken into account for these calculations.

Figure 2.13 clearly demonstrates a good agreement between distances derived from ${ }^{1} \mathrm{H},{ }^{7} \mathrm{Li}$ HOESY experiments and the crystal structures for compounds $\left[(\mathrm{DME}) \mathrm{Li}\left(\mathrm{C}_{4} \mathrm{H}_{3} \mathrm{~S}\right)\right]_{2} 2 \mathrm{c}$ and 
[(PMDETA $\left.) \mathrm{Li}\left(\mathrm{C}_{4} \mathrm{H}_{3} \mathrm{~S}\right)\right]$ 2e. A similar plot was obtained for [(TMEDA $\left.) \mathrm{Li}\left(\mathrm{C}_{4} \mathrm{H}_{3} \mathrm{~S}\right)\right]_{2}$ 2d. That means that the structures of $\mathbf{2 c - e}$ are retained upon dissolving in toluene. Small deviations may originate from intramolecular motions that affect the $\mathrm{HOE}$ in solution, e.g. a partial dissociation of one side-arm of the chelating donor molecules. This could be verified for the DME-coordinated 2-thienylltihium (2c) by evaluation of the corresponding RDCs, for which a fit to an ensemble of different conformers indicated significant population of the conformer with one side-arm of the DME moiety dissociated. The different behaviour of 2b already indicated by ${ }^{1} \mathrm{H}$-DOSY NMR is mirrored by the ${ }^{1} \mathrm{H},{ }^{7} \mathrm{Li}-\mathrm{HOESY}$ data: Compared to what is expected from the crystal data the cross peaks belonging to the THF protons are much too weak. Again, this would be explained by a partial dissociation of THF molecules from a dimeric core or, alternatively, THF molecules rapidly exchanging between the vertices of a tetrameric core and free solution. With regard to the latter explanation it should be noted that $\Sigma r(\mathrm{H} \cdots \mathrm{Li})^{-6}$ for a conceivable tetrameric structure $\left[(\mathrm{THF}) \mathrm{Li}\left(\mathrm{C}_{4} \mathrm{H}_{3} \mathrm{~S}\right)\right]_{4}$ is in very good congruency with the measured HOESY data (Figure 2.14). However, no unambiguous conclusion can be drawn from this data.
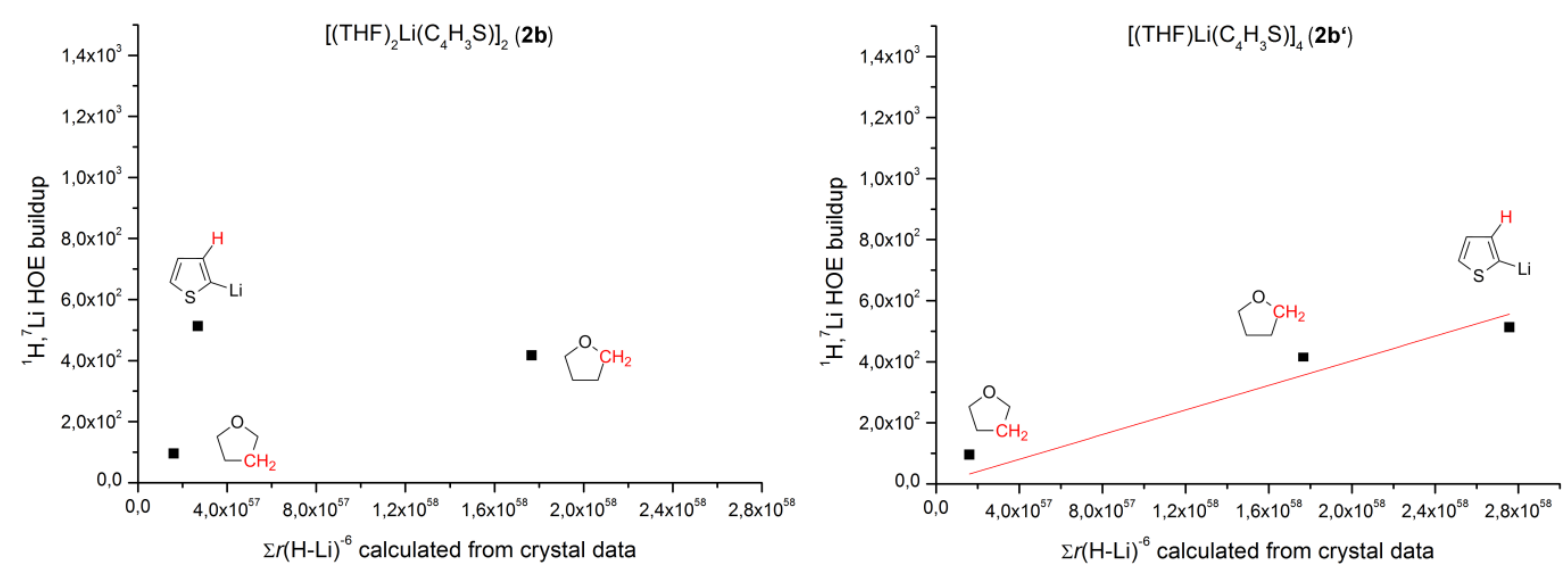

Figure 2.14: Comparison of the ${ }^{1} \mathrm{H},{ }^{7} \mathrm{Li}-\mathrm{HOE}$ build-up with the calculated $\Sigma r(\mathrm{H} \cdots \mathrm{Li})^{-6}$ terms from crystal data of $\mathbf{2 b}$ (left) and for a hypothetical tetrasolvated tetramer $\mathbf{2} \mathbf{b}$ ' (right). Disorders in the crystal have been taken into account for these calculations.

Summarizing, the distance relation of $\mathrm{Li} \cdots \mathrm{H}$ NOEs is an effective tool to gain insight into the aggregation state of $\mathbf{2 a - e}$ in a non-donating solvent. Thereby, it could be confirmed that the structures of $\mathbf{2 c - e}$ are retained in toluene solution. Additionally, EXSY investigations showed that the nitrogen-donor base containing compounds $\mathbf{2 d}$ and $\mathbf{2 e}$ exchange to the signals of non-lithiated thiophene. This is explained by exchange of the deuterium atom by a hydrogen atom via lithiation of toluene molecules followed by protonation. Finally, it would be interesting to investigate whether the structures $\mathbf{2 a - e}$ are also retained in different solvents and to further explore the enhanced basicity of the $\mathrm{N}$-donor-base-coordinated lithium compounds. 


\subsubsection{Interim Conclusion}

The previous two subchapters underline that two-dimensional NMR spectroscopy, in this case DOSY and HOESY experiments, represent a really powerful tool to gain information about the aggregation of organometallic compounds in solution. The two molecular systems show a range of interesting phenomena. The mixed compound $[t \mathrm{BuLi}]_{4} \cdot 4\left[\mathrm{Me}_{2} \mathrm{NC}_{6} \mathrm{H}_{4} \mathrm{Li}\right]_{4}$ shows a highly dynamic behaviour forming at least five clearly distinguishable aggregates, whereas in the case of 2-thienyllithium retention with respect to the solid state structures could be verified unambiguously in three cases. Additionally, it becomes clear that lithium chemistry substantially benefits from combining methods and risking a view beyond one's nose. This could be underlined by the two presented projects where X-ray diffraction as the most important tool for the analysis of the solid state structure provided a firm starting point offering deep knowledge about the bonding situation and arrangement. From here, different NMR spectroscopic approaches were combined in order to deepen the understanding of the structure in solution and possible dynamic behaviour. This could be accomplished and even reactivity properties (oxygen versus nitrogen-donor) could be identified experimentally. Although important and powerful, the ${ }^{1} \mathrm{H},{ }^{7} \mathrm{Li}$-HOESY experiments are rather time consuming and a crystal structure or geometry optimized structural model is necessary in most cases. Therefore, it would also be an interesting aim to search for a more straightforward and less time consuming analytical method to shed more light on the behaviour of organometallic compounds in solution. 


\subsection{Structure Elucidation by Anisotropic NMR Experiments}

\subsubsection{Employment of Polystyrene Gels for the Analysis of Organolithium Compounds}

The importance of structure determination in organometallic chemistry in general and organolithium chemistry in particular has been given attention to in the previous chapters. Conventional two-dimensional NMR experiments could be successfully implemented to ascertain the structure of these highly reactive species in solution. However, the request for a more straightforward method to judge on the aggregation state arose during these studies. In the following, the results of an analysis of organolithium compounds by alignment in swollen polystyrene gels are discussed.

A first challenge for the analysis of organolithium compounds by means of anisotropic NMR experiments was the design of an alignment medium that would withstand those highly reactive compounds. The aforementioned strain induced alignment in a gel (SAG) method seemed most promising as one of the first employed polymer systems was polystyrene (PS), which displays no functional groups that could possibly be attacked. Furthermore, swelling of the gel in toluene is feasible. Apart from the fact that toluene is chemically rather inert, it closely resembles the polymer environment and allows the observation of controlled deaggregation by addition of stoichiometric amounts of Lewis bases (e.g. lithiated thiophene in chapter 2.1.2). Although benzene would allow the same and is even less attractive to organolithiums, toluene is preferred over benzene due to a lower melting point which is important because low temperature studies have proven to be vital for exact structure elucidation.

\subsubsection{Polymer Synthesis}

Cross-linked polymer networks can be obtained by radical polymerization of styrene with the linker divinylbenzene (DVB) according to the procedure reported by Luy et al. (Scheme 2.3). ${ }^{[89 \mathrm{~d}]}$

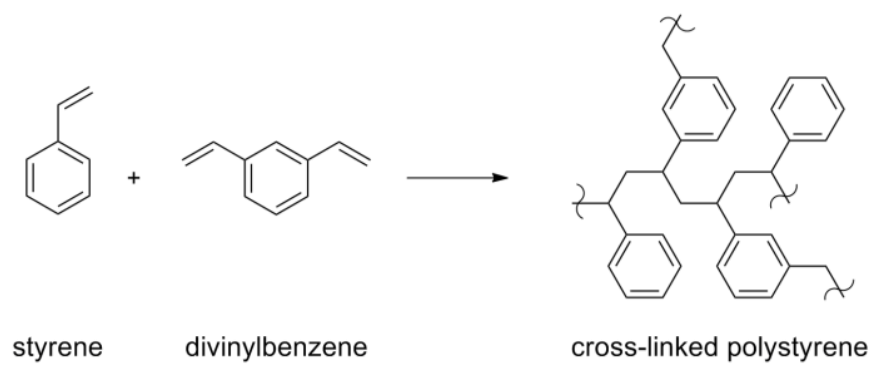

Scheme 2.3: Reaction of styrene and divinylbenzene to form cross-linked polystyrene networks. 
According to this protocol, the monomers were filtered, distilled and finally degassed prior to the polymerization. In the following, the initiator (e.g. 2,2'-azobis(2methylpropionitrile) (AIBN)) was added and the mixture transferred to glass tubes with inner diameters of 3.4 and $4.0 \mathrm{~mm}$ that were treated with chlorotrimethylsilane and dichlorodimethylsilane. The polymerization was run for two days at $60^{\circ} \mathrm{C}$.

However, in the above presented synthesis, several problems had to be faced (Figure 2.15): First, enclosed gas-bubbles resulted in little homogeneous material while in second attempt, microcrystalline polymeric material (so-called "popcorn polymers") was obtained that proved to be totally unusable (middle picture). While the former can be explained by the polymerization temperature below the glass point of styrene, formation of popcorn polymers was
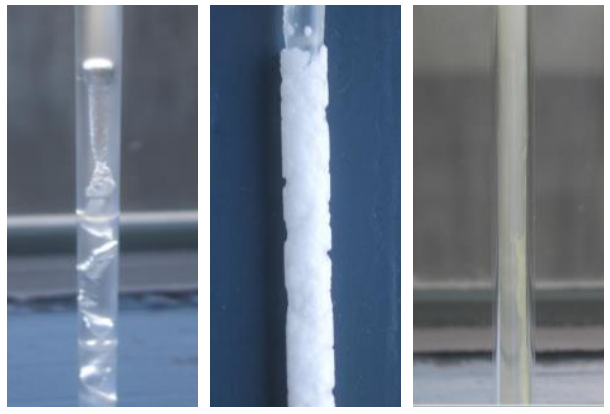

Figure 2.15: PS with enclosed bubbles (left), popcorn polymer (middle) and a homogeneous sample (right). not observed by Luy et al. A possible explanation could be the quality of the styrene employed for this synthesis. A very high quality (99\%) was used in this case, while the literature protocol was carried out with only moderately pure styrene (80\%). The remaining $20 \%$ might act as a solvent ensuring continuous flexibility while the pure styrene polymerization yield rigid material that can easily crack. In order to obtain homogeneous polystyrene sticks, the original procedure was therefore slightly modified by abstaining from the use of an initiator. Instead, the polymerization process was thermally induced. The thermal self-initiation of styrene (at temperatures above $100^{\circ} \mathrm{C}$ near $T_{\mathrm{g}}$ ) is well known and its mechanism has been verified by computational methods only recently (Scheme 2.4). ${ }^{[157]}$

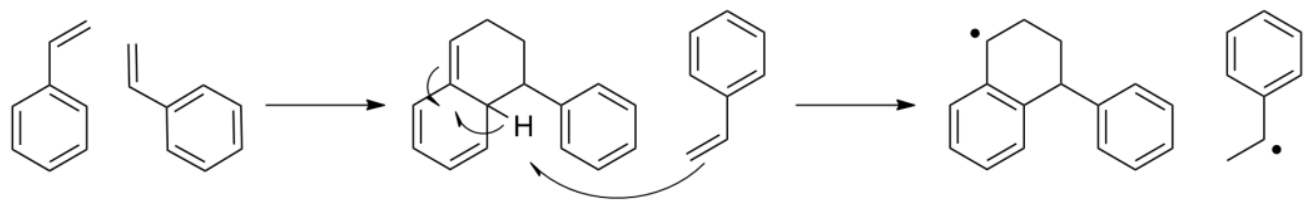

Scheme 2.4: Mechanism of the thermal initiation of styrene supported by quantum chemical calculations.

It is likely that part of the enclosed gas-bubbles were caused by decomposition of the applied initiator, for example AIBN yielding $\mathrm{N}_{2}$. Therefore, initiating the polymerization by heat is beneficial in two ways: to avoid gas-bubbles and to obtain very pure polymeric material. The latter aspect is also crucial for the application to reactive compounds. Popcorn polymers have been the subject of various investigations, nevertheless, the exact conditions that lead to the formation of this large-scale inhomogeneity are not fully 
understood. ${ }^{[158]}$ By modifying the reaction conditions it was possible to single out a temperature of about $115^{\circ} \mathrm{C}$ and a linker concentration of $0.6 \mathrm{vol} \%$ or below as best settings for the polystyrene stick preparation. With this optimized proceeding over $90 \%$ of a $15 \mathrm{~cm}$ polystyrene stick could be applied to the successful measurement of anisotropic NMR parameters. Generally, different approaches for the sample preparation are conceivable:

(1) The stick can be positioned at the bottom of the NMR sample tube.

(2) The stick can be adjusted to the centre of the NMR coil completely surrounded by the solvent.

(a) Swelling can be done in the pure solvent. The compounds are added subsequently.

(b) The polymer stick can be swollen directly in a solution of the analyte.

Best experiences were made with a PS stick of $3.8 \mathrm{~mm}$ inner diameter and approach (2), because compared to (1) breaking of the gels during the swelling process was extremely seldom. Furthermore, approach (b) was generally preferred over (a) as it saves the additional time the analyte requires to diffuse into the already swollen gel. However, an advantage of (a) is that the gel can be washed during the swelling process by exchanging the residual solvent on top of the gel.

Similar to the report of Luy et al. the swelling was monitored by acquiring ${ }^{2} \mathrm{H}$ NMR spectra of the corresponding deuterated solvents. This is exemplarily depicted for toluene- $d_{8}$ in Figure 2.16 but can be equally observed for THF- $d_{8}, \mathrm{CDCl}_{3}$ and benzene- $d_{6}$. The sharp signals of the isotropic spectrum broaden significantly and show a well resolved quadrupolar splitting for equilibrated samples after several days of swelling. If the resulting anisotropic spectrum is free of remaining isotropic signal and plateaus or tailing of the measured signals, the polymer environment is considered sufficiently homogeneous. Defects of the polymer can thus be easily detected. The trends $\|$

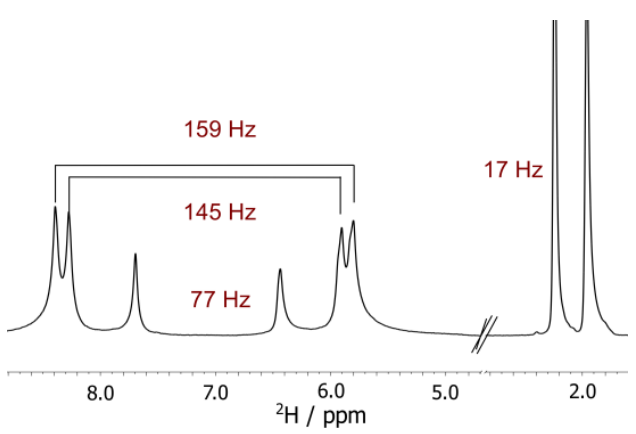

Figure 2.16: Isotropic and anisotropic ${ }^{2} \mathrm{H}$ NMR spectrum of toluene- $d_{8}$. described in the above-mentioned publication ${ }^{[89 \mathrm{~d}]}$ could also be confirmed, namely an observed increase of the quadrupolar splitting with 
increasing diameter of the polymer stick and increasing linker concentration. This can be directly explained by the fact that the splitting in the ${ }^{2} \mathrm{H}$ NMR spectrum is caused by the partial alignment of the deuterated solvent molecules inside the cavities of the gel. The higher the linker concentration the smaller the resulting cavities due to a higher number of connecting points. If the diameter of the polymer stick approaches the diameter of the NMR sample tube, swelling in the horizontal direction becomes effectively impossible and the resulting cavities become strongly ellipsoidally shaped, inducing a higher degree of order.
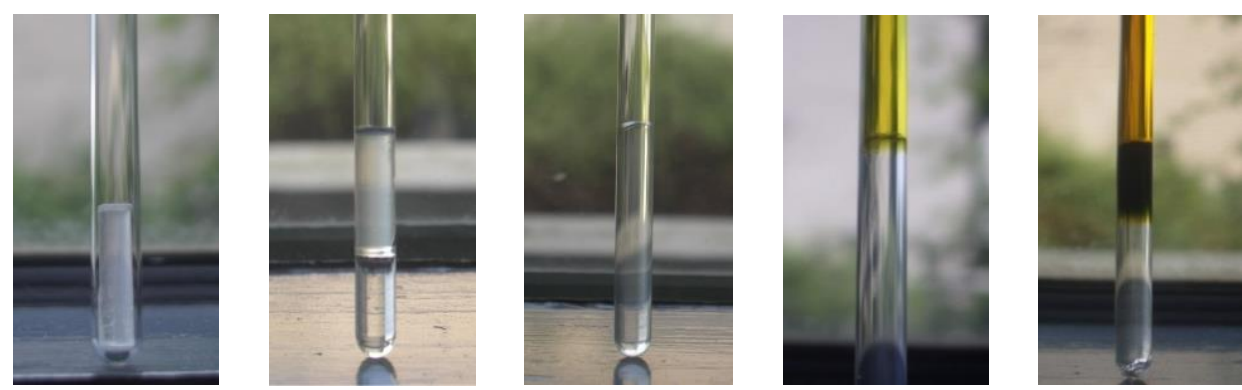

Figure 2.17: Homogeneous swelling of a PS gel and subsequent addition of $n \mathrm{BuLi}$.

The swelling was carried out with toluene- $d_{8}$ as solvent, because neither the toluene molecules nor the chemically related polystyrene network are supposed to react in this case. After the preparation of homogeneous gels, hexameric $n$-butyllithium ( $n \mathrm{BuLi}$ ) was added as a first reactive compound. Unfortunately, a slow destruction of the polymeric material was observed (Figure 2.17, right). Therefore, the quality and possible impurities of the polymer had to be investigated more thoroughly. For this purpose, the recording of standard ${ }^{1} \mathrm{H}$ NMR spectra was not very suitable as can be easily seen in Figure 2.18 (bottom).

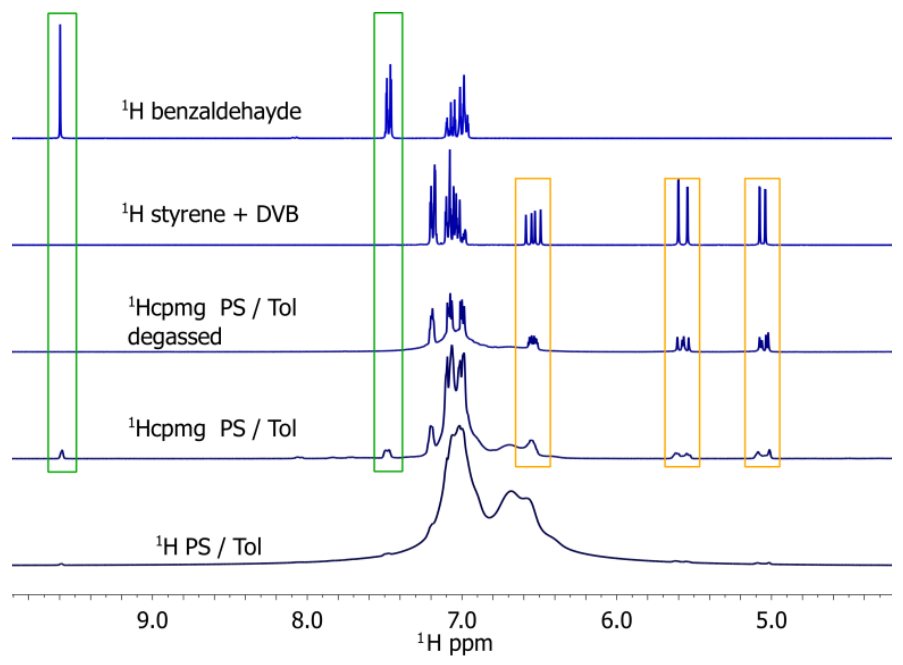

Figure 2.18: Aromatic region of ${ }^{1} \mathrm{H}$ and ${ }^{1} \mathrm{H}-\mathrm{CPMG}$ NMR spectra of various swollen PS sticks and reference samples in toluene- $d_{8}$. 
The standard ${ }^{1} \mathrm{H}$ NMR spectrum of a polystyrene gel swollen in toluene- $d_{8}$ is dominated by the very intense and broad polymer signals at around $2 \mathrm{ppm}$ and $7 \mathrm{ppm}$, so that impurities and even the residual solvent signal cannot be distinguished. To bypass this difficulty, ${ }^{1} \mathrm{H}-$ CPMG (Carr-Purcell-Meiboom-Gill) spectra were recorded that facilitate the identification of these components by suppressing the immense polymer signals. This experiment is based on differences between the transversal relaxation time $T_{2}$ and thereby allows filtering of signals belonging to high molecular weight molecules. Consequently, the next spectrum (Figure 2.18, second from bottom) reveals the presence of additional signal sets that could be identified as benzaldehyde (green) and remaining monomer/linker (orange) from the corresponding reference samples. Both compounds might readily react with organolithiums. The oxidation of styrene to benzaldehyde is a common issue in the polymerization of styrene and its kinetics. ${ }^{[159]}$ In the following, all reactants were thoroughly degassed by several freeze-pump-thaw cycles prior to use and only handled under Schlenk conditions (for more details see experimental section). The sticks prepared in this way are free of benzaldehyde and tolerate the diffusion of highly reactive compounds although styrene and DVB are still present in the sample (see 2.2.1.2). Although longer polymerization times slightly decrease the amount of styrene, a considerable amount remains inside the polymer stick. Further reduction of remaining monomer by storing the sticks in a vacuum stove at $50{ }^{\circ} \mathrm{C}$ for five days resulted in only little decreased shares of styrene.

As a final check on the polymers with respect to its general applicability for the SAG method, a PS / Tol- $d_{8}$ sample with (-)-menthol (3) was prepared as a proof of principle. RDCs were extracted from the F2-dimension of coupled ${ }^{13} \mathrm{C}$-HSQC spectra and fitted to a geometry optimized structure of menthol with the help of the program MSpin. ${ }^{[160]}$ The results of this calculation are depicted in Figure 2.18. As output, a set of calculated RDCs is given as well as a quality factor (Cornilescu's Q, equation (2-1)) indicating the agreement of proposed structure and measured RDCs.

$$
Q=\sqrt{\frac{\sum\left(D_{i}^{\exp }-D_{j}^{\text {calc }}\right)^{2}}{\sum D_{i}^{\exp }}}
$$

A number below 0.2 is normally regarded as sufficient for structure validation. For menthol, a value of 0.1 could be accomplished, and the straight line observed in a comparison of the experimental and calculated values (Figure 2.19, right) further underlines the agreement. 

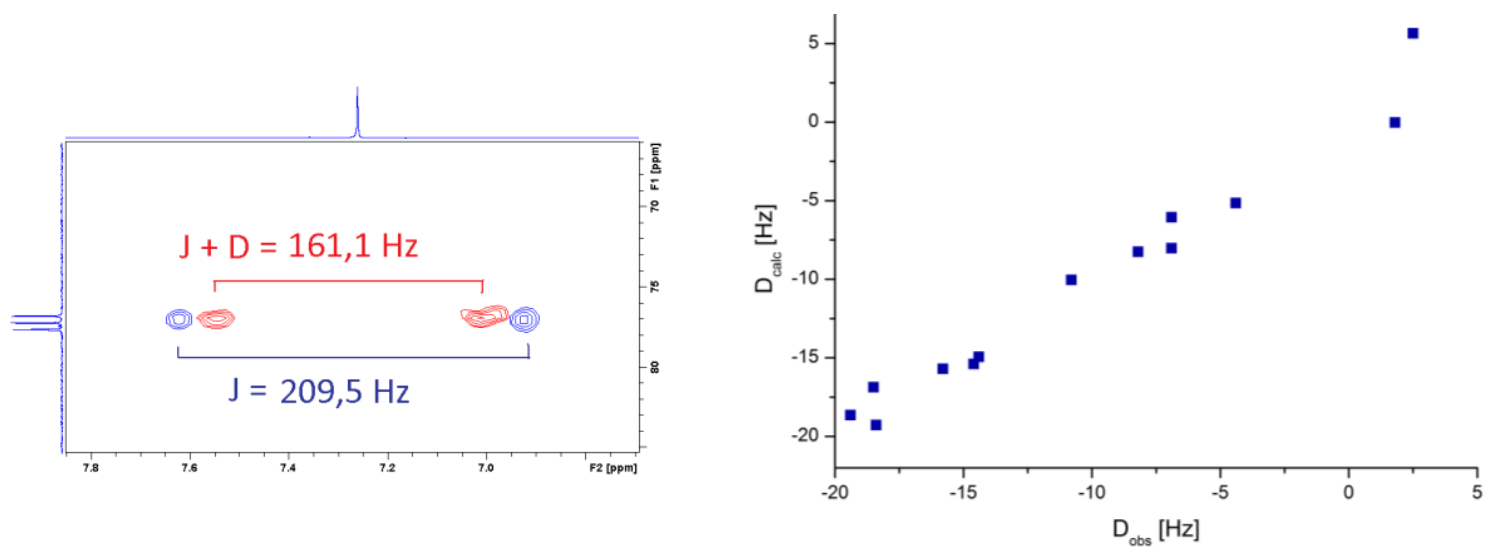

Figure 2.19: Overlay of coupled ${ }^{13} \mathrm{C}-\mathrm{HSQC}$ spectra of toluene- $d_{8}$ in isotropic (blue) and anisotropic (red) environment (left) to illustrate the extraction of RDCs. The experimental RDCs of menthol were plotted vs. those calculated by the program MSpin ${ }^{[160]}$ (right). The small deviation indicates the quality of the fit.

Therefore, it can be deduced that these very pure and thermally polymerized PS gels display the same fundamental characteristics as those introduced by Luy and co-workers. Furthermore, they even tolerate the harsh conditions arising from the presence of highly reactive organolithium compounds which will be discussed explicitly in the following.

\subsubsection{Analysis of Text Book Lithium Compounds}

To apply the polystyrene sticks $(3.8 \mathrm{~mm}, 0.2-0.5 \mathrm{vol} \%$ linker) for the analysis of organolithium compounds in solution, they were stored in an argon dry box and always handled under inert gas atmosphere. Samples were prepared by dissolving the corresponding compound in the deuterated solvent and placing the sticks to swell directly in this solution. Either all the solution $(0.5 \mathrm{~mL})$ may be placed inside the NMR sample tube and a PS stick can be adjusted in the centre of the NMR coil or $0.2 \mathrm{~mL}$ of this mixture can be filled into the tube first, followed by adjustment of the stick at the surface of the solvent an subsequent addition of the remaining $0.3 \mathrm{~mL}$ of the prepared solution. The first of these approaches is only feasible if the diameter of the stick is close to the ID of the NMR sample tube. Holding the tube horizontally until the swelling has started proved to be beneficial for the positioning of the stick. In many cases, diffusion of coloured compounds into the polymer could be directly monitored optically (Figure 2.20). The presence of analytes inside the gel was confirmed

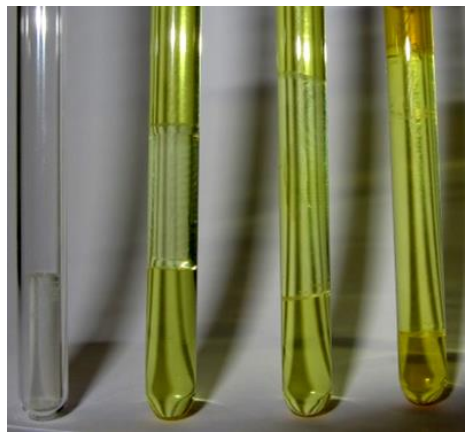

Figure 2.20: Polystyrene stick inside a $5 \mathrm{~mm}$ NMR tube as a dry stick, directly after preparation and after three and seven days of swelling (from left to right). via ${ }^{1} \mathrm{H}-\mathrm{CPMG}$ spectra, while the quality of the gel was monitored by the quadrupolar splitting in the ${ }^{2} \mathrm{H}$ NMR spectrum. 
Scheme 2.5 displays a range of text book lithium compounds that were investigated in swollen polystyrene gels.

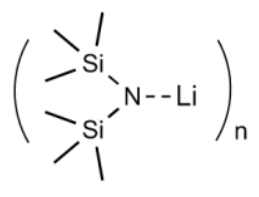

$\begin{array}{ll} & n \\ 4 \text { in tol } & 2 \\ 4 \text { in THF } & 2 / 1\end{array}$

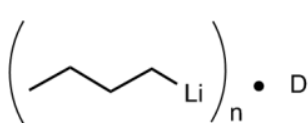

D

$\begin{array}{ccc}\mathbf{5 a} & & 6 \\ \mathbf{5 b} & \mathrm{Et}_{2} \mathrm{O} & 4 \\ \mathbf{5 c} & \mathrm{THF} & 4 / 2 \\ \mathbf{5 d} & \text { PMDETA } & 1\end{array}$

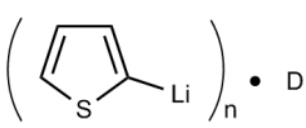

D

2a $\mathrm{Et}_{2} \mathrm{O} \quad 4$

$\begin{array}{lll}\text { 2c } & \text { DME } & 2 \\ 2 \text { e } & \text { PMDETA } & 1\end{array}$

Scheme 2.5: Common organolithium and lithium amide reagents used in this work. The degree of aggregation $n$ depends on the solvent or stoichiometric donor base D. Aggregation: $n=6$ hexamer, $n=4$ tetramer, $n=2$ dimer, $n=1$ monomer. If two numbers are given for $n$, an equilibrium is present.

The method was first tested on lithium hexamethyldisilazane (LiHMDS; 4), which is only moderately reactive, well characterized and known to form a $(\mathrm{NLi})_{2}$ cyclic dimer in toluene solution. ${ }^{[161]}$ In the isotropic ${ }^{7} \mathrm{Li}$ NMR spectrum recorded at room temperature, a singlet with a chemical shift $\delta=1.1 \mathrm{ppm}$ is observed. After preparation of the anisotropic sample (0.2 vol\% DVB) according to the procedure reported above, ${ }^{1} \mathrm{H}-\mathrm{CPMG}$ and ${ }^{7} \mathrm{Li} \mathrm{NMR}$ spectra were recorded. The ${ }^{1} \mathrm{H}-\mathrm{CPMG}$ spectrum unequivocally confirmed the presence of LiHMDS inside the polymer gel, and interestingly, the ${ }^{7} \mathrm{Li}$ NMR spectrum displayed a triplet with an integral ratio of 3:4:3 and a quadrupolar splitting of $75 \mathrm{~Hz}$ could be observed (Figure 2.21, left). The outer lines of the triplet are broader than the central line (17 vs. $11 \mathrm{~Hz}$ ), which is due to differential transverse relaxation of the individual transitions in a spin $I=3 / 2$ nucleus ${ }^{[162]}$ and in agreement with earlier findings (see chapter 1.2.2.2). ${ }^{[104]}$
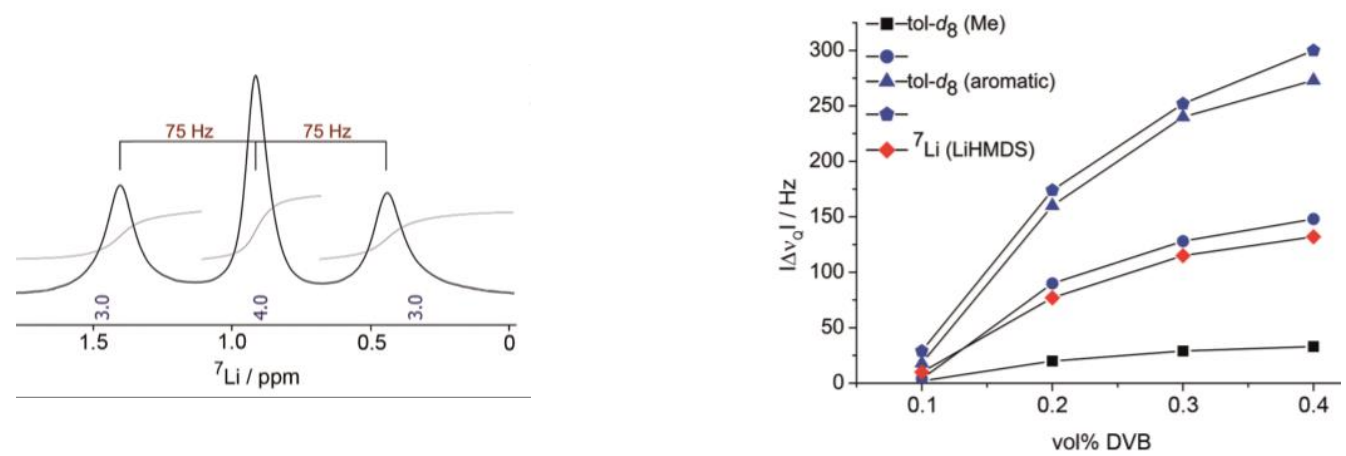

Figure 2.21: Left: ${ }^{7} \mathrm{Li}$ quadrupolar splitting of LiHMDS (4) in PS / toluene- $d_{8}$ at room temperature using PS with 0.2 vol\% DVB; right: dependence of the ${ }^{2} \mathrm{H}$ and ${ }^{7} \mathrm{Li}$ quadrupolar splittings on the linker concentration in vol\%. Note that only the absolute values of $\Delta v_{\mathrm{Q}}$ are obtained.

Analogous to the trend reported by Luy et al., ${ }^{[89 b]}$ the ${ }^{7} \mathrm{Li}$ quadrupolar splitting increases with increasing linker concentration (Figure 2.21, right). This scalability of the splitting thus presents an alternative to the use of a stretching apparatus. ${ }^{[94 \mathrm{~d}, 104]}$ An assuring aspect of 
these first experiments was the high stability of all samples that were intact for at least four weeks. Besides, the observed splittings were highly reproducible and independent of the LiHMDS concentration in the range of $25-150 \mathrm{mg}$ per $0.5 \mathrm{~mL}$ of deuterated solvent.

In a next step, we were interested whether our PS gels would also be compatible with and show observable ${ }^{7} \mathrm{Li}$ quadrupolar splittings at lower temperatures which are typically employed when using reactive organolithium reagents. Therefore, a swollen polymer containing LiHMDS (4) in toluene- $d_{8}$ was stepwise cooled down to $228 \mathrm{~K}$. The measured ${ }^{7} \mathrm{Li}$ splitting increases slightly from $107 \mathrm{~Hz}(298 \mathrm{~K})$ to about $190 \mathrm{~Hz}(248 \mathrm{~K})$; at temperatures below $238 \mathrm{~K}$ the outer lines become too broad to be observable (Figure 2.22, left). Line broadening was likewise observed in the ${ }^{2} \mathrm{H}$ NMR spectra and, as for the ${ }^{7} \mathrm{Li}$ NMR spectra, found to be fully reversible (Figure 2.22, right).
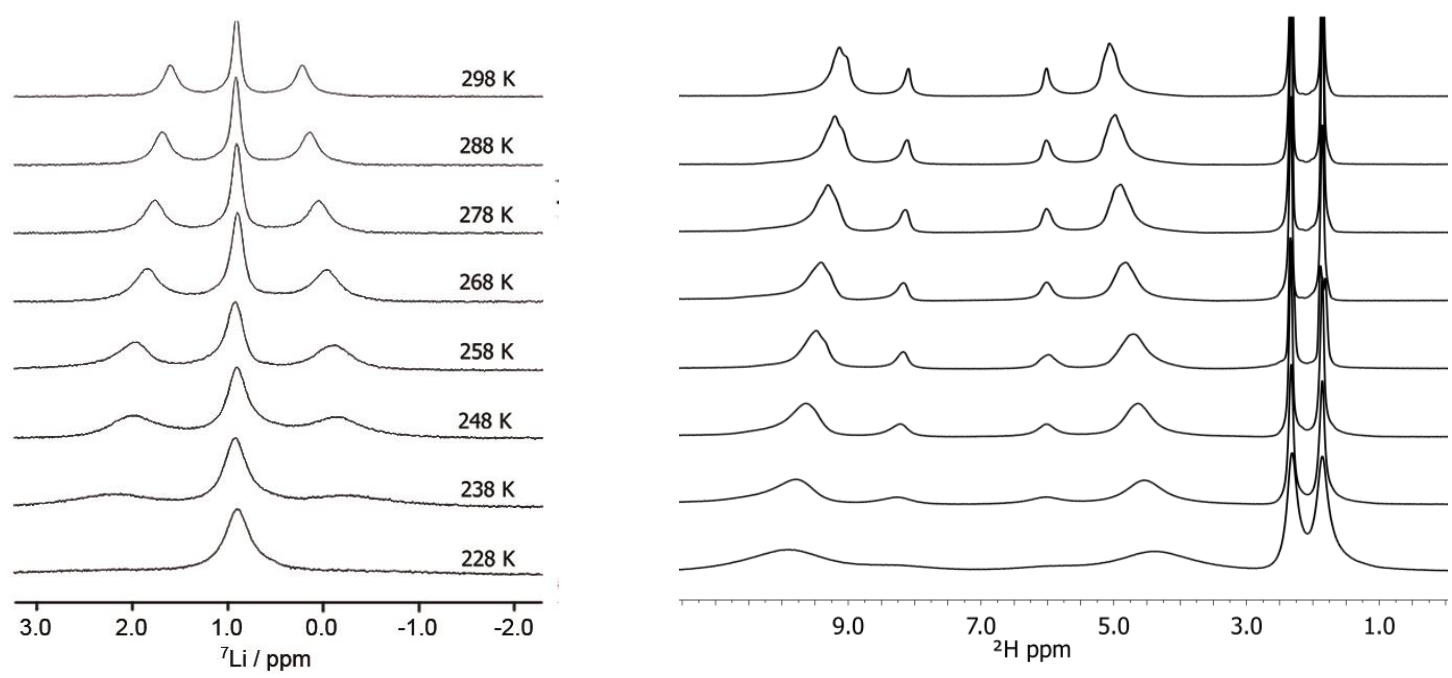

Figure 2.22: Variable temperature spectra of LiHMDS (4) in PS (0.3 vol\% DVB) / toluene- $d_{8}$ (left) and variable temperature ${ }^{2} \mathrm{H}$ NMR spectra of the same sample (right).

The compatibility of PS gels with low temperatures enables the investigation of equilibria between different aggregation states which are frequently observed for many organolithiums: For example, dissolving the test compound LiHMDS in THF- $d_{8}$, where it forms an equilibrium between THF-solvated monomeric and dimeric species, ${ }^{[163]}$ resulted in separate signals in the isotropic ${ }^{7} \mathrm{Li}$ NMR spectrum below $273 \mathrm{~K}$ (Figure 2.23, left). Inside the PS gel, each of the two lines splits into a quadrupolar triplet with RQCs of 71 and $45 \mathrm{~Hz}$ for the dimer and monomer, respectively (Figure 2.23, right). The higher quadrupolar coupling in the dimer can be rationalized in terms of two instead of one negatively polarized nitrogen atom in the lithium environment. The monomer-dimer integral ratio of 2:1 in isotropic solution is almost reversed in the anisotropic sample, which may be due to the less polar environment of the polystyrene gel. 

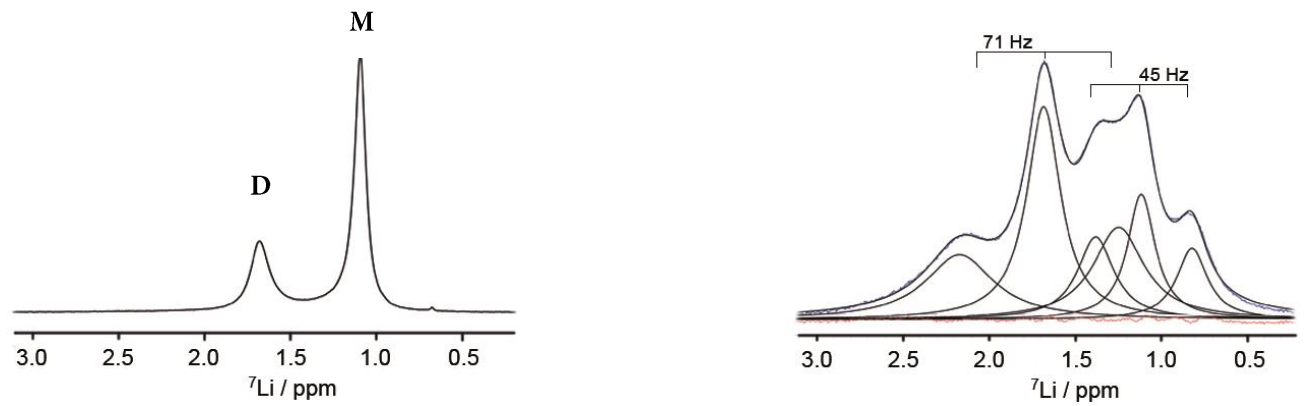

Figure 2.23: Isotropic (left) and anisotropic (right) spectrum of 4 in THF- $d_{8}$ at $273 \mathrm{~K}$. The anisotropic spectrum (black line) was fitted to two quadrupolar triplets with overall six Lorentzians (gray lines). The fit residual is also shown (red line).

After providing proof of principle the pure polymer sticks were applied to $n$-butyllithium (5a-d) and 2-thienyllithium (2a, c, e) solutions in toluene containing stoichiometric amounts of donor bases. In contrast to diethyl ether or THF as solvents, ${ }^{[164]}$ these solutions with only stoichiometric amounts of Lewis bases are remarkably stable at room temperature, and the desired organolithium compound can be detected inside the PS gel without major side-products by ${ }^{1} \mathrm{H}-\mathrm{CPMG}$ spectra (e.g. 3c in Figure 2.24). The ether-coordinated 2thienyllithium tetramer is the only structure not included in this anisotropic NMR spectroscopic study due to its low overall solubility and strong line broadening that impedes an accurate analysis of the ${ }^{7} \mathrm{Li}$ inside PS / toluene- $d_{8}$ gels.

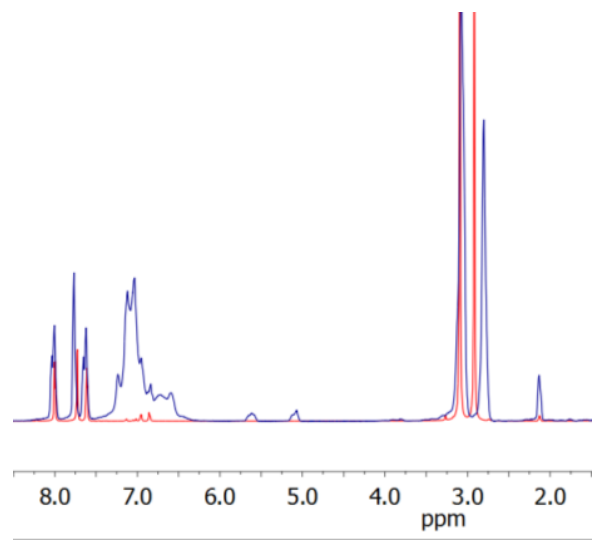
Analogous to LiHMDS (4), ${ }^{7} \mathrm{Li}$ quadrupolar splittings were also observed for $n \mathrm{BuLi}$ in the presence of PMDETA (5d), and for 2-

Figure 2.24: Overlay of the ${ }^{1} \mathrm{H}$ NMR spectrum of $3 \mathbf{c}$ (ThiLi.DME) in toluene- $d_{8}$ (red) and the ${ }^{1} \mathrm{H}-\mathrm{CPMG}$ spectrumof $3 \mathrm{c}$ in PS / toluene- $d_{8}$ (blue). thienyllithium in the presence of $\operatorname{DME}(2 c)$ and PMDETA (2e) (Figure 2.25). 2c forms a (CLi) $)_{2}$ dimeric structure similar to that of $\mathbf{4}^{[151]}$ while $2 \mathbf{e}$ (and presumably $\mathbf{5} \mathbf{d}$ ) ${ }^{[165]}$ are monomeric. 


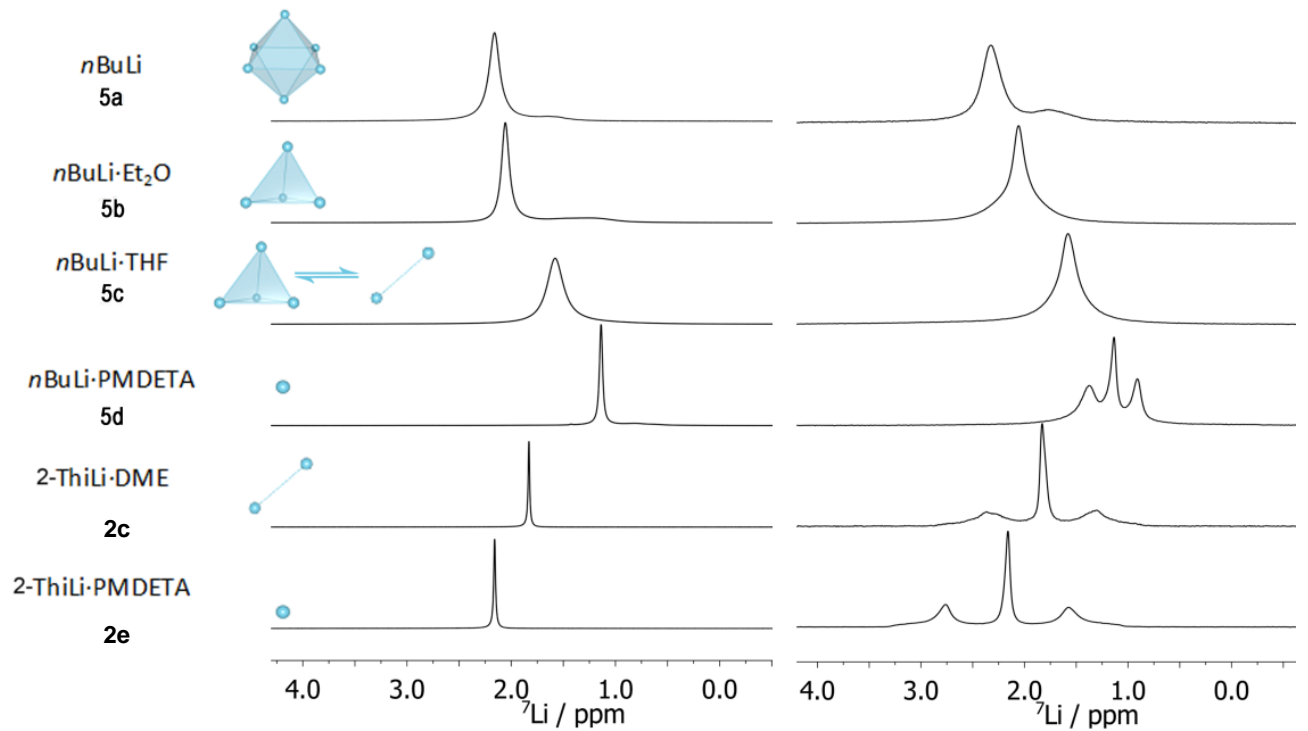

Figure 2.25: Structures and room-temperature ${ }^{7} \operatorname{Li}$ NMR spectra of $n \operatorname{BuLi}(\mathbf{5 a}-\mathbf{d})$ and 2-ThiLi (2a,c,e) in the presence of stoichiometric amounts of donor bases. The isotropic spectra (left) were recorded in toluene- $d_{8}$. The anisotropic spectra (right) were recorded in PS / toluene- $d_{8}$.

In contrast, no ${ }^{7} \mathrm{Li}$ quadrupolar splitting is observed for $n \mathrm{BuLi}$ without additional donor bases (5a) or in the presence of $\mathrm{Et}_{2} \mathrm{O}(\mathbf{5 b})$ and THF (5c), or for 2-ThiLi with $\mathrm{Et}_{2} \mathrm{O}$ (2a, not shown). Both, the isotropic and anisotropic spectrum, resemble each other except for some line broadening of the anisotropic spectrum because of a slight inhomogeneity of the gel and the applied magnetic field. In three of these four cases the organolithium compound forms higher aggregates containing $\mathrm{Li}_{4}$ tetrahedra $\left(\mathbf{5} \mathbf{b},{ }^{[28,166]} \mathbf{2} \mathbf{a}\right)^{[151]}$ or $\mathrm{Li}_{6}$ octahedra $(\mathbf{5 a})^{[23]}$ such that electric-field gradients (efg) within the molecule are effectively averaged to zero. In $\mathbf{5 c}$, the efg is averaged to zero owing to fast equilibration between tetrameric and dimeric species. Although RQCs have been observed in tetrahedral molecules as a result of rotational-vibrational coupling ${ }^{[167]}$ they are generally at least one order of magnitude smaller in size and therefore do not interfere with the detection of herein reported quadrupolar splittings.

Thus, discrimination between aggregates of higher symmetry (tetrameric, hexameric) and those of lower symmetry (dimeric, monomeric) can be easily accomplished by measuring a single ${ }^{7} \mathrm{Li}$ NMR spectrum in an anisotropic environment. This is a very straightforward and time-saving approach for the direct, qualitative determination of aggregation states in lithiumorganic chemistry and may be further extended to other quadrupolar nuclei (for example ${ }^{6} \mathrm{Li},{ }^{10 / 11} \mathrm{~B},{ }^{23} \mathrm{Na}$ ). It should be noted that the quadrupolar splitting also highly depends on the orientation of the entire molecule inside the gel (see equation (1-5) of the introduction) and its surrounding charges (i.e. nitrogen-containing Lewis bases vs. oxygencontaining ethers). ${ }^{[158]}$ Therefore, a single quadrupolar coupling constant extracted in Hertz does not yet contain substantial structural information. Nevertheless, in the future, 
prediction of RQCs may become feasible as a result of constantly increasing knowledge about efg tensor parameters from solid-state NMR spectroscopy data, ${ }^{[168]}{ }^{7} \mathrm{Li}$ relaxation times $^{[169]}$ and DFT calculations. ${ }^{[170]}$ This combined knowledge might then lead to an advanced, more accurate differentiation permitting even the discrimination, for instance, between dimers and monomers.

Furthermore, the purity and performance of the polystyrene sticks used in this work might be improved even more by modifying the polymer network. The rather long preparation time due to the swelling might be significantly shortened by using higher temperatures, higher pressure or recycling polymer gels by rinsing with neat solvents. On the other hand, the utilization of higher swelling temperatures might not always be beneficial for the herein analysed, highly reactive class of organometallic compounds. Here, one could thus think about a further optimization of low temperature handling of the gels implying not only measurement but also swelling at low temperatures. These last aspects will be treated systematically in chapter 2.2.2, whereas deeper insight into different polymer networks will be given in the later course of this work (chapter 2.2.3). 


\subsubsection{Slice-Selective NMR Experiments}

In the previous chapters, the successful application of chemically inert polymer gels to highly reactive organometallic compounds for the extraction of anisotropic NMR parameters could be demonstrated. The degree of alignment induced thereby is easily scalable, for example, by change of the cross-linker concentration. In general, this gel approach relies on

(1) the swelling of the polymer by the solvent and

(2) diffusion of the particular molecule into the gel.

Both processes may take several days before the sample is sufficiently homogeneous for high-precision measurements of the desired anisotropic parameter. An illustration of this process is shown below (Figure 2.26).

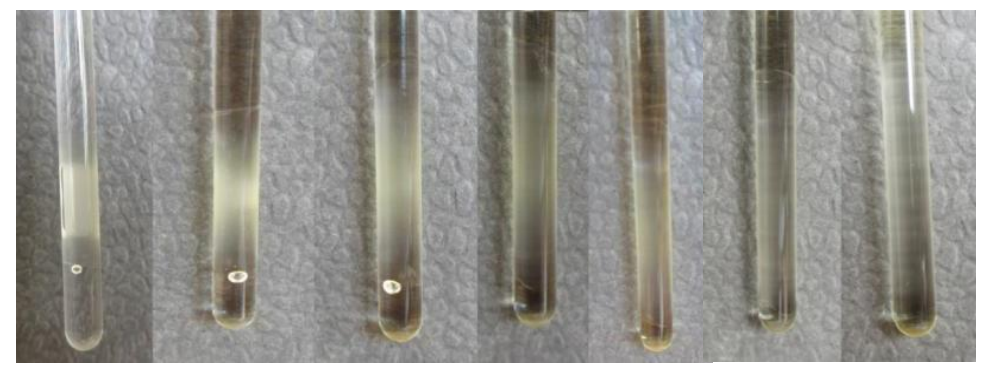

Figure 2.26: Photographs of a 0.2 vol\% DVB sample swollen by a solution of 4 in toluene- $d_{8}$. From left to right: $3 \mathrm{~h}, 1,2,3,4,7,12$ days after sample preparation. An air bubble (up to day 2) marks the bottom of the gel.

As described above, the solvent ${ }^{2} \mathrm{H}$ NMR is commonly employed as an estimate of the sample homogeneity. Nevertheless, this estimate is rather crude. A possibility to directly monitor the swelling and diffusion inside the gel with spatial resolution would be of great benefit as it allows an improved detection of gel defects and sample inhomogeneity which often make the extraction of anisotropic parameters difficult. Additional knowledge of the properties and behaviour of the polymer upon addition of strain might also turn out to be advantageous for a further optimization of polymer systems used for strain induced alignment in a gel. In the following, an easy and non-invasive method that relies on selective excitation and thus detection of nuclear spins in individual thin horizontal slices of the sample is presented. The method was applied to cross-linked PS swollen by a solution of lithium hexamethyldisilazane 4 in toluene- $d_{8}$, which was already used for the proof of principle in the preceding chapter due to its moderate reactivity and known dimeric structure in hydrocarbon solution. 


\subsubsection{Technique}

Slice-selective excitation (SSE) is currently used in a wide variety of Magnetic Resonance Imaging (MRI) techniques. ${ }^{[171]}$ It is realized by a soft radiofrequency (RF) pulse applied in the presence of a magnetic field gradient (Figure 2.27a). ${ }^{[172]}$ All resonance frequencies experience an offset $v_{z}$ during the field gradient $G$ that depends on the vertical deviation $z$ from the centre of the gradient coil ( $\gamma=$ nuclear gyromagnetic ratio):

$$
v_{z}=\frac{\gamma G z}{2 \pi}
$$

Hence, a horizontal slice of the sample centred at $z$ and with a thickness given by

$$
\Delta z=\frac{2 \pi}{\gamma G} \Delta v
$$

can be selectively excited by applying a soft pulse with a frequency offset $v_{z}$ and a bandwidth $\Delta v$. As depicted in the same figure, the field gradient is switched off before acquisition. Therefore, all spins are detected with their "normal" resonance frequencies.
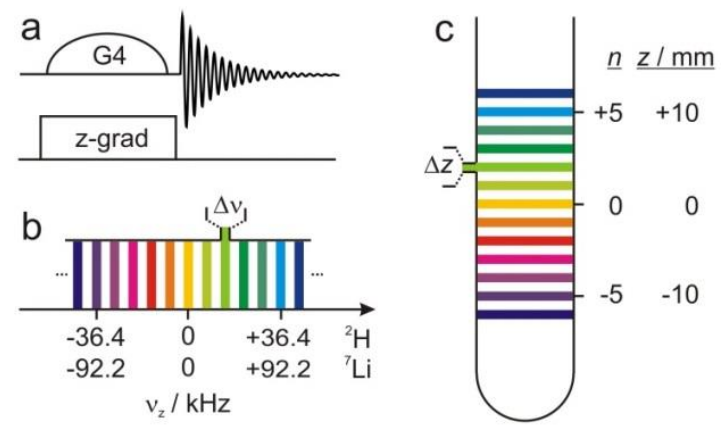

Figure 2.27: Slice-selective ${ }^{2} \mathrm{H}$ and ${ }^{7} \mathrm{Li}$ NMR spectroscopy. a) Pulse scheme. The z-gradient was applied at the maximum available strength $(55.7 \mathrm{G} / \mathrm{cm})$. b) Chosen ${ }^{2} \mathrm{H}$ and ${ }^{7} \mathrm{Li}$ offsets. c) Location of the 13 slices $(-6 \leq n \leq$ $+6)$ relative to the centre of the gradient coil $(z=0)$.

In total, 13 slices $(-6 \leq n \leq+6)$ in a distance of $2 \mathrm{~mm}$ were excited for both nuclei. In this case, $n=0$ represents the centre of the gradient coil and six slices are measured in each direction (Figure 2.27b-c). A $90^{\circ}$ G4 Gaussian cascade ${ }^{[173]}$ with a duration of $1 \mathrm{~ms}$ and a bandwidth of $9.4 \mathrm{kHz}$ on ${ }^{7} \mathrm{Li}$ was employed and resulted in a thickness of $1.02 \mathrm{~mm}$, while the thickness of the ${ }^{2} \mathrm{H}$ slices $(0.55 \mathrm{~mm})$ was somewhat reduced due to the low $(3.5 \mathrm{~W})$ power output of the lock amplifier resulting in a much longer $90^{\circ}$ pulse $(4.7 \mathrm{~ms}$, corresponding to a bandwidth of $2.0 \mathrm{kHz}$ ). In the following, the colour code is utilized to characterize the different slices is in accordance with the colouring of the corresponding ${ }^{2} \mathrm{H}$ and ${ }^{7} \mathrm{Li}$ NMR spectra.

The sign of $G$ and the position of the centre of the gradient was verified by recording sliceselective ${ }^{2} \mathrm{H}$ NMR spectra with a sample of silica layered with toluene- $d_{8}$ (Figure 2.28). All spectra were recorded with 16 scans and long recycle delays to ensure the magnetization 
had fully relaxed prior to excitation. Of the 13 measured slices, only the uppermost six slices show signals in the ${ }^{2} \mathrm{H}$ NMR spectrum thus corresponding to the liquid toluene- $d_{8}$ phase on top of the silica (positive $G$ and $n)$. The central slice $(n=0)$ and all slices with negative $n$ values did not show any signal.

\begin{tabular}{l|lllll} 
SPOFF 43680 \\
\hline SPOFF 36400
\end{tabular}

Figure 2.28: Slice-selective ${ }^{2} \mathrm{H}$ NMR spectra of toluene- $d_{8}$ layered over silica. $v_{\mathrm{s}}$ is given in $\mathrm{Hz}$.

Interestingly, only little signal is detected from the outermost slices which is also observed for all investigated anisotropic samples. To investigate this loss in intensity a liquid sample of lithium hexamethyldisilazane 4 in toluene- $d_{8}$ was analysed by slice-selective ${ }^{2} \mathrm{H}$ and ${ }^{7} \mathrm{Li}$ experiments. The ${ }^{2} \mathrm{H}$ integrals corresponding to the deuterated solvent were extracted and plotted as a function of the slice (Figure 2.29).
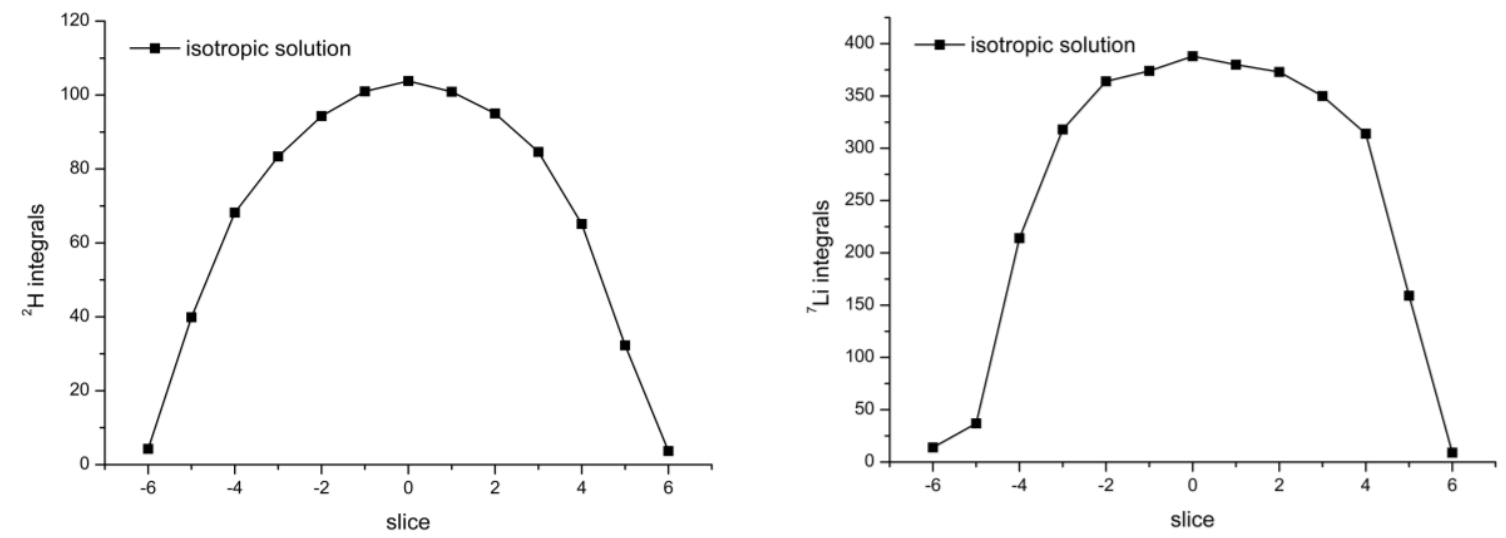

Figure 2.29: Methyl $-{ }^{2} \mathrm{H}$ (left, inner coil) and ${ }^{7} \mathrm{Li}$ (right, outer coil) integrals of a $0.3 \mathrm{M}$ liquid sample of 4 in toluene- $d_{8}$.

The symmetric profiles obtained in this way show the same tendencies as for the layered sample and can thus be ascribed to the limited dimensions of the RF coil, which implies 
that the outermost slices are located outside the active region. In the following, the integral profiles of the isotropic sample are further used for referencing the integrals obtained in an anisotropic environment, resulting in time-dependent concentration profiles (see chapter 2.2.2.2).

To monitor swelling with spatial resolution, polymer sticks (length: $1 \mathrm{~cm}, \phi 3.8 \mathrm{~mm}$ ) consisting of mixtures of styrene with $0.2,0.3$ and $0.4 \mathrm{vol} \%$ divinylbenzene using the protocol systematically described in chapter 2.2.1.1 were prepared. The sticks were placed in a conventional $5 \mathrm{~mm}(\phi 4.2 \mathrm{~mm})$ NMR sample tube filled with $0.2 \mathrm{~mL}$ of 4 ( $0.3 \mathrm{M}$ based on the dimer) in toluene- $d_{8}$ and adjusted to the centre of the RF coil. Afterwards, another $0.3 \mathrm{~mL}$ of the solution was added such that diffusion of solvent and $\mathbf{4}$ simultaneously occurred from both ends of the polymer. In addition to the cross-linker concentration, the swelling temperature was varied for a cross-linker concentration of $0.3 \mathrm{vol} \%$ resulting in data sets for $+40,+22,+4$ and $-20^{\circ} \mathrm{C}$, respectively. The slice-selective ${ }^{2} \mathrm{H}$ and ${ }^{7} \mathrm{Li} \mathrm{NMR}$ experiments (approximately $30 \mathrm{~min}$ in total) were carried out at room temperature and the samples subsequently stored again at the corresponding swelling temperature. Two hours after preparation, the first measurement was carried out and each sample was monitored daily over a period of 11-12 days.

\subsubsection{Basic Information from Slice-selective ${ }^{2} \mathrm{H}$ and ${ }^{7} \mathrm{Li}$ NMR Spectroscopy}

In this subchapter, several general results of the slice-selective ${ }^{2} \mathrm{H}$ and ${ }^{7} \mathrm{Li} \mathrm{NMR}$ experiments of anisotropic samples of 4 in PS / toluene- $d_{8}$ are presented in order to provide an overview of the general significance of this method for polymer gel analysis. A detailed evaluation of the swelling characteristics with regard to the linker concentration and the swelling temperature is depicted in the following subchapters.

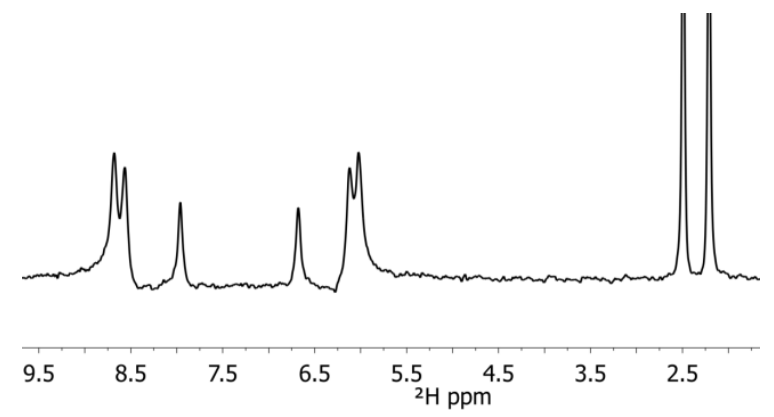

Figure 2.30: Slice-selective $(z=0){ }^{2} \mathrm{H}$ NMR spectrum after 12 days of swelling $(0.2$ vol\% DVB). The phase of $o$ - and $m-{ }^{2} \mathrm{H}$ quadrupolar doublets is distorted by about $\pm 20 \mathrm{~Hz}$, whereas the phase distortion of the methyl${ }^{2} \mathrm{H}$ quadrupolar doublet $\left(\Delta v_{\mathrm{Q}}=17 \mathrm{~Hz}\right)$ is about $\pm 2^{\circ} \mathrm{Hz}$. 
In case of the ${ }^{2} \mathrm{H}$ NMR spectrum, only the signal of the methyl group is displayed, because the aromatic signals suffer from significant phase distortions due to coupling evolution during the gradient (G4) pulse (Figure 2.30).

All six samples (three cross-linker concentrations and four distinct temperatures) were prepared in an identical manner as described above and subjected to slice-selective ${ }^{2} \mathrm{H}$ and ${ }^{7} \mathrm{Li}$ NMR experiments. For the sample with $0.2 \mathrm{vol} \%$ DVB and swelling at room temperature the stacked spectra obtained at different swelling times are shown in Figure 2.31.

First of all, these spectra offer a quick and direct insight into the overall homogeneity of the sample at a particular swelling time. Initially (after $3 \mathrm{~h}$ of swelling, Figure 2.31 top) the outer slices show singlets corresponding to the supernatant clear liquid phases and only small amounts of the deuterated solvent or the lithiumamide are detected within the central region of the yet non-swollen polymer sticks. This nicely agrees with the photographs
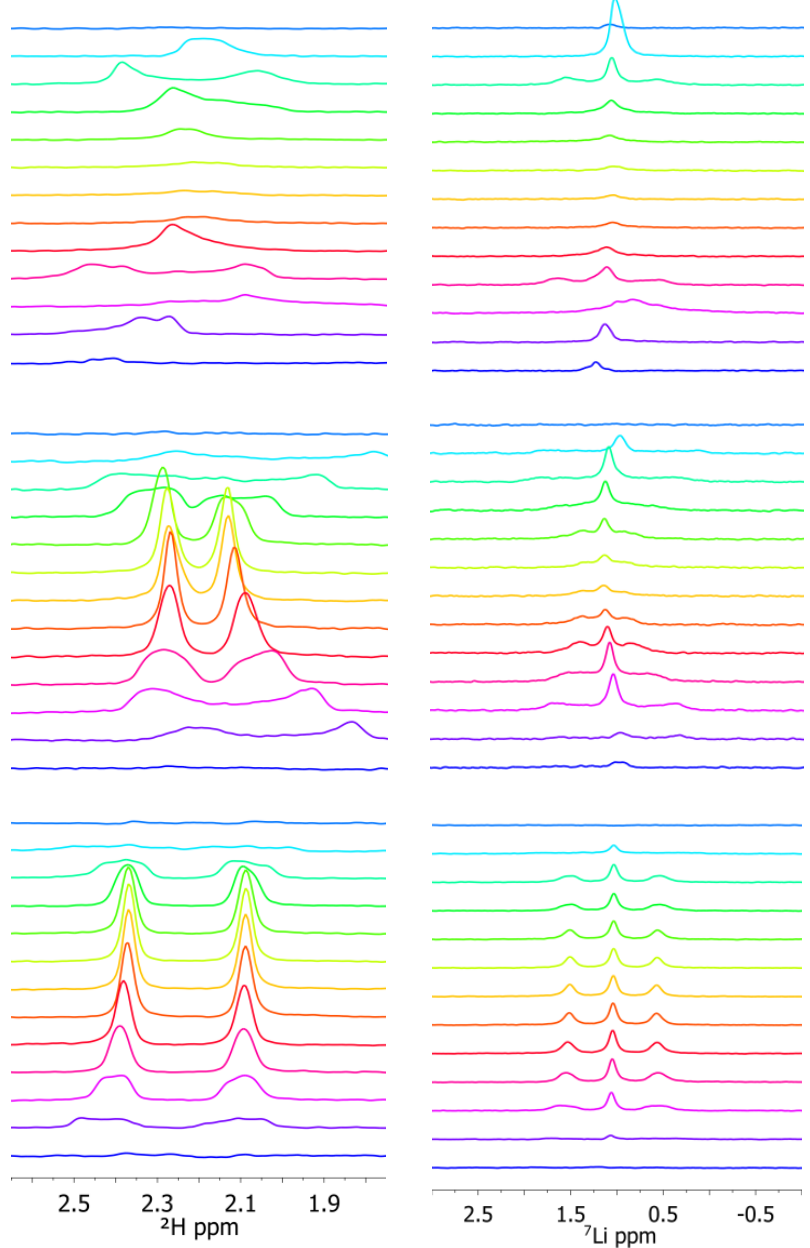

Figure 2.31: Slice-selective ${ }^{2} \mathrm{H}$ (left) and ${ }^{7} \mathrm{Li}$ (right) $\mathrm{NMR}$ spectra of 4 in toluene- $d_{8}$ diffusing into a PS stick (0.2 vol\%) after $3 \mathrm{~h}, 3 \mathrm{~d}$ and $12 \mathrm{~d}$ (top to bottom). (Figure 2.26) exhibiting at first a slightly turbid boundary layer where the solvent starts intruding the polymer network. After three days, an almost uniformly transparent polymer gel is obtained, in agreement with the corresponding ${ }^{2} \mathrm{H}$ NMR spectra (middle) which likewise confirm the visual impression that the polymer is now completely penetrated by the solvent. Due to the axial strain imposed by the walls of the sample tube, all molecules inside the gel are partially aligned with respect to the magnetic field causing the corresponding ${ }^{2} \mathrm{H}$ NMR signals originating from inside the gel to appear as quadrupolar doublets, while quadrupolar triplets are observed for ${ }^{7} \mathrm{Li}(\mathrm{I}=3 / 2)$ with splittings that were found to be generally proportional to that of methyl- ${ }^{2} \mathrm{H}\left(v_{\mathrm{Q}}\left({ }^{7} \mathrm{Li}\right) \approx 4 \times v_{\mathrm{Q}}\left({ }^{2} \mathrm{H}\right)\right)$. Furthermore, the splittings and hence the strain notably diverge across the sample as the ${ }^{2} \mathrm{H}$ doublets in the centre of the gel are comparatively 
narrow with uniform splittings ( $9 \mathrm{~Hz}$ for the methyl group), whereas the splittings in the outer slices are less homogeneous and display larger quadrupolar splittings. Compared to ${ }^{2} \mathrm{H}$, however, the ${ }^{7} \mathrm{Li}$ signal inside the polymer builds up much slower, likely due to slower diffusion of $4\left(M_{\mathrm{r}}=334\right)$ compared to toluene $\left(M_{\mathrm{r}}=92\right) \cdot{ }^{[174]}$ In terms of molecular movement, this implies, that primarily the solvent enters the gradually entangling and extending polymer network, followed by diffusion of the dissolved analyte. After 12 days, the homogeneous region has grown in size with increased splittings $(17 \mathrm{~Hz})$. Nevertheless, heterogeneity is clearly still present to some extent towards the ends of the RF coil.

For the series of slice-selective ${ }^{2} \mathrm{H}$ and ${ }^{7} \mathrm{Li}$ NMR experiments, the quadrupolar splittings in Hertz can be extracted for each corresponding swelling time. Plotting the splittings with respect to the excited slice leads to time-dependent quadrupolar coupling profiles as depicted in Figure 2.32.
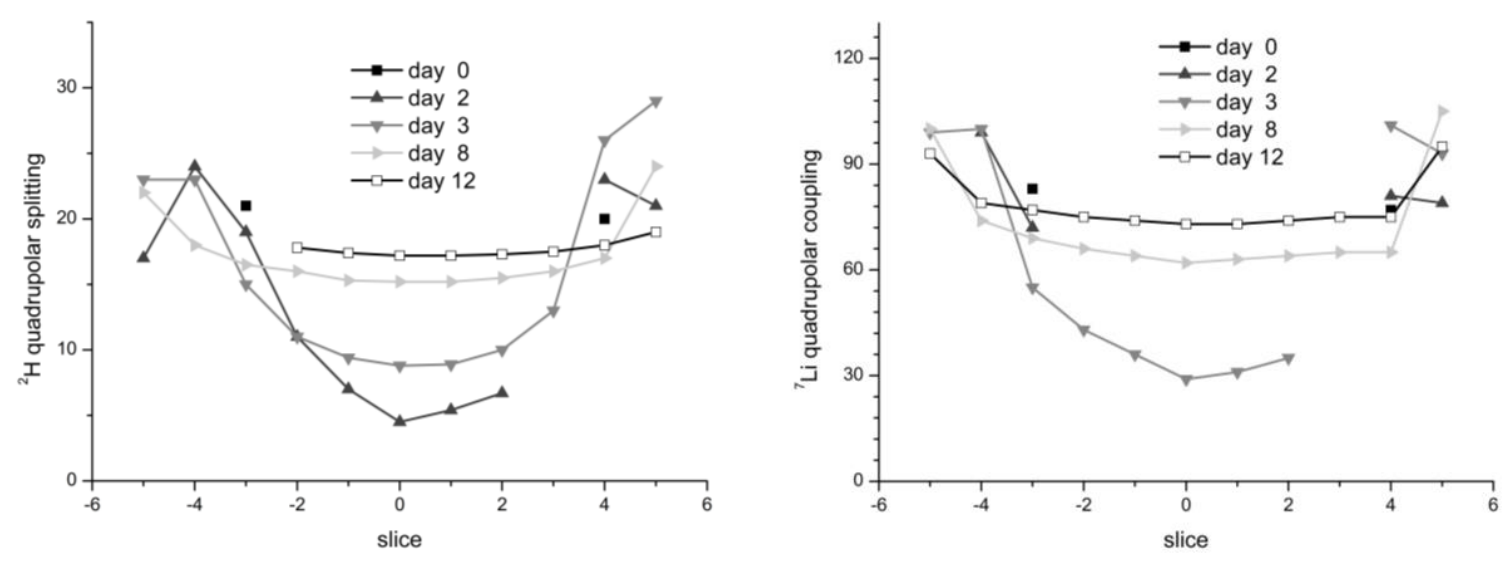

Figure 2.32: Methyl- ${ }^{2} \mathrm{H}$ (left) and ${ }^{7} \mathrm{Li}$ (right) quadrupolar coupling profiles obtained from a 0.2 vol\% DVB sample of 4 in PS / toluene- $d_{8}$ measured $0,2,3,8$ and 12 days after sample preparation.

This provides an alternative illustration of the above-described time-resolved swelling behaviour including the faster diffusion of the solvent molecules, for which ${ }^{2} \mathrm{H}$ quadrupolar splittings are observed already at day two compared to day three in case of lithium. The gradual approach of the equilibrium swelling state is also very well represented by these plots.

By integration of the methyl- ${ }^{2} \mathrm{H}$ and ${ }^{7} \mathrm{Li}$ signals in the individual slices and referencing with the isotropic sample of $\mathbf{4}$ (Figure 2.29) time-dependent concentration profiles can be obtained that illustrate the corresponding ${ }^{2} \mathrm{H}$ and ${ }^{7} \mathrm{Li}$ concentrations as a function of the time as well as the position within the sample. 

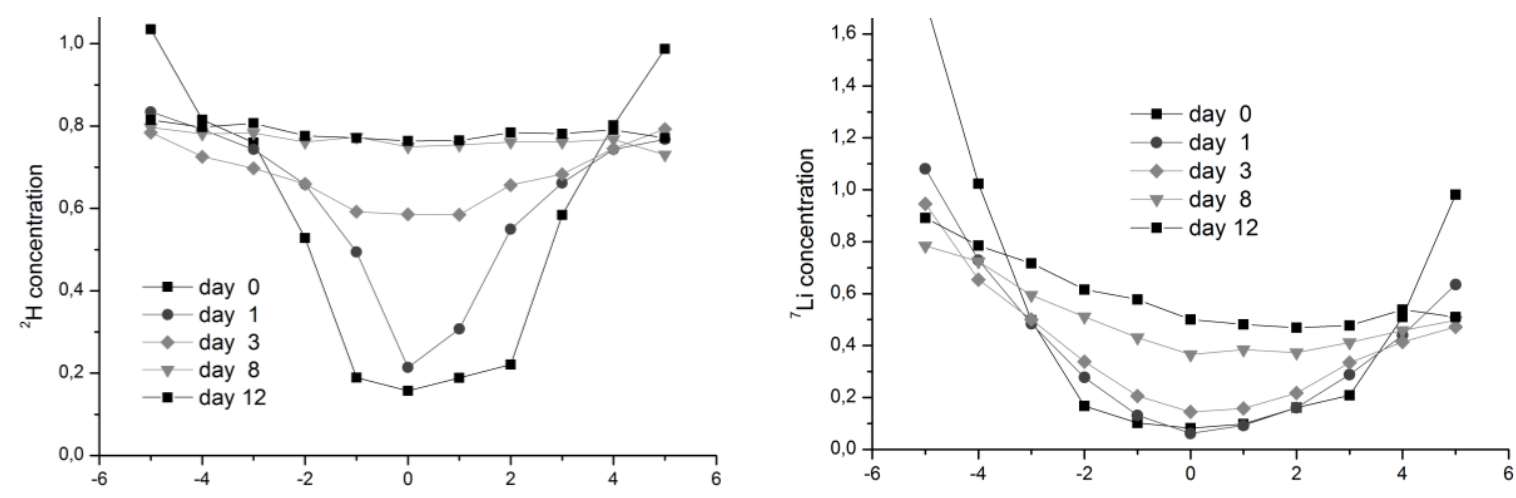

Figure 2.33: Time-dependent concentration profiles of toluene- $d_{8}$ and 4 measured $0,2,3,8$ and 12 days after sample preparation.

While the solvent concentration in the gel quickly reaches saturation at about $80 \%$, the concentration of 4 is only about $50 \%$ and still far from equilibrium even after 12 days. This further underlines the above-presented impression that diffusion of $\mathbf{4}$ into the gel is delayed with respect to toluene. A saturation of $80 \%$ in the case of ${ }^{2} \mathrm{H}$ also represents a sensible value, because the measured sample volume contains a considerable amount of polymeric material in contrast to the purely liquid phase measured as a reference.

Apart from the slice-selective experiments, standard ${ }^{2} \mathrm{H}$ and ${ }^{7} \mathrm{Li}$ NMR spectra with uniform excitation were recorded (Figure 2.34).
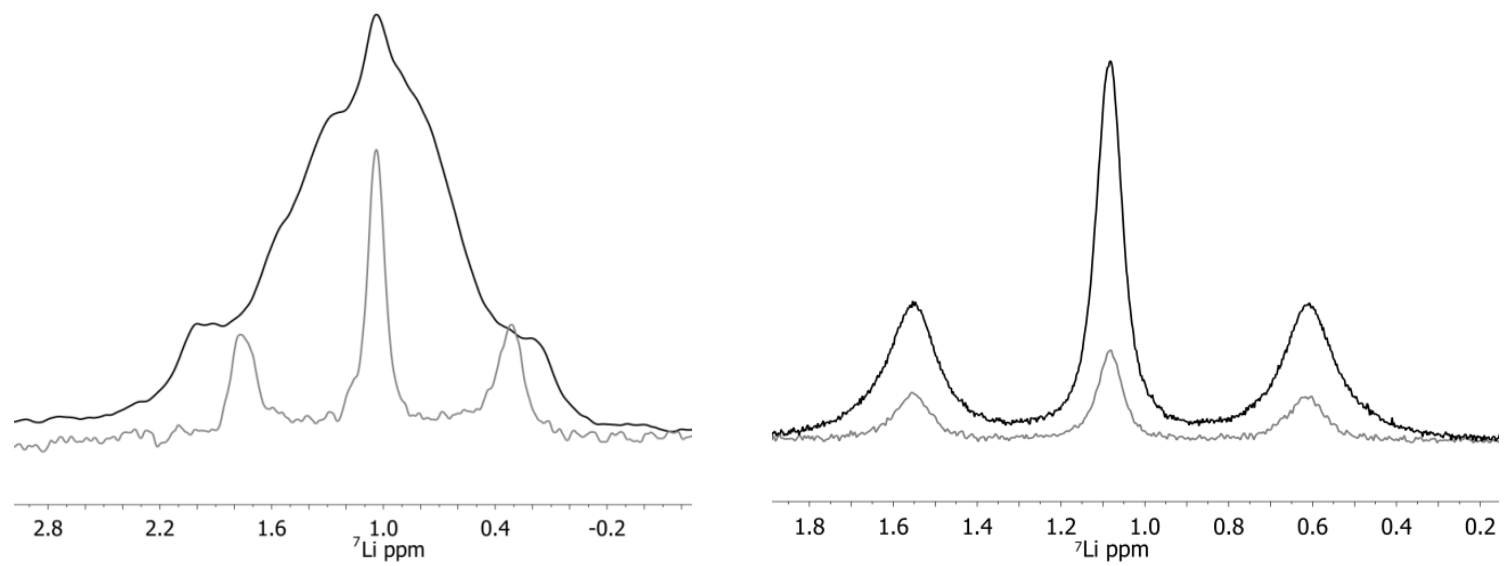

Figure 2.34: Overlay of slice-selective (grey) and uniform (black) ${ }^{7} \mathrm{Li}$ NMR spectra two days $(z=4$, left) and 12 days ( $z=0$, right) after sample preparation.

Remarkably, a quadrupolar triplet in the ${ }^{7} \mathrm{Li}$ NMR spectrum of the slice $n=+4$ can be observed as early as two days after sample preparation, whereas in the uniformly excited spectrum which represents the sum over all slices, a broad undefined peak appears. Allowing the sample to equilibrate for 12 days yields slice-selective (middle slice, $n=0$ ) and uniform ${ }^{7} \mathrm{Li}$ NMR spectra as depicted on the right side of Figure 2.34. Therein, the satellite lines for the central slice are still 30-40\% narrower than for a uniformly excited spectrum 
which might be beneficial for signal separation in case of equilibria or strongly overlapping signals.

Comparison of the uniformly excited ${ }^{7} \mathrm{Li} \mathrm{NMR}$ spectrum of a sample recorded after eleven days of swelling with the sum of all slice-selective experiments recorded under analogous conditions results in Figure 2.35. The red, uniform spectrum seems to envelope the summed slice-selective spectra, which is owed to the fact that the 13 slices (à $0.55 \mathrm{~mm}\left({ }^{2} \mathrm{H}\right)$ and $\left.1.02 \mathrm{~mm}\left({ }^{7} \mathrm{Li}\right)\right)$ in the distance of $2 \mathrm{~mm}$ do not enclose the complete sample volume. In summary, slice-selective analysis presents a rich source of information and will be applied in the following to study the dependency of the swelling on cross-linking and temperature.

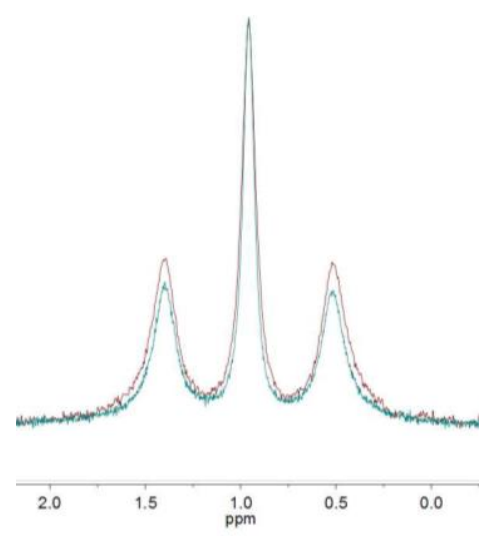

Figure 2.35: Overlay of the sum of all slice-selective experiments (green) and a uniform (red) ${ }^{7} \mathrm{Li}$ NMR spectrum eleven days after sample preparation.

\subsubsection{Dependency of the Swelling on the Cross-linking}

By plotting quadrupolar splittings and concentrations for a given slice as a function of time, build-up curves are obtained for varying cross-linker concentrations $(0.2,0.3$ and $0.4 \mathrm{vol} \%$ ). The measurement for a linker concentration of $0.4 \mathrm{vol} \%$ had to be repeated due to a crack in the gel causing a complete collapse of the quadrupolar splittings in the nearby slices. As expected, the three samples show increased quadrupolar splitting build-up rates and also higher values on the final day (Figure 2.36, top). The measured ${ }^{2} \mathrm{H}$ quadrupolar splittings are 17, 24 and $34 \mathrm{~Hz}$, respectively while for ${ }^{7} \mathrm{Li} 68,85$ and $133 \mathrm{~Hz}$ were obtained. The small bend observed directly after preparation (day 0) is caused by the smaller diameter of the polymer stick with respect to the inner diameter of the NMR sample tube allowing minor swellings in the horizontal direction. The thereby induced strain would cause negative quadrupolar splittings, which cannot be distinguished herein because the acquired experiments only yield absolute $\Delta v_{\mathrm{Q}}$ values. 

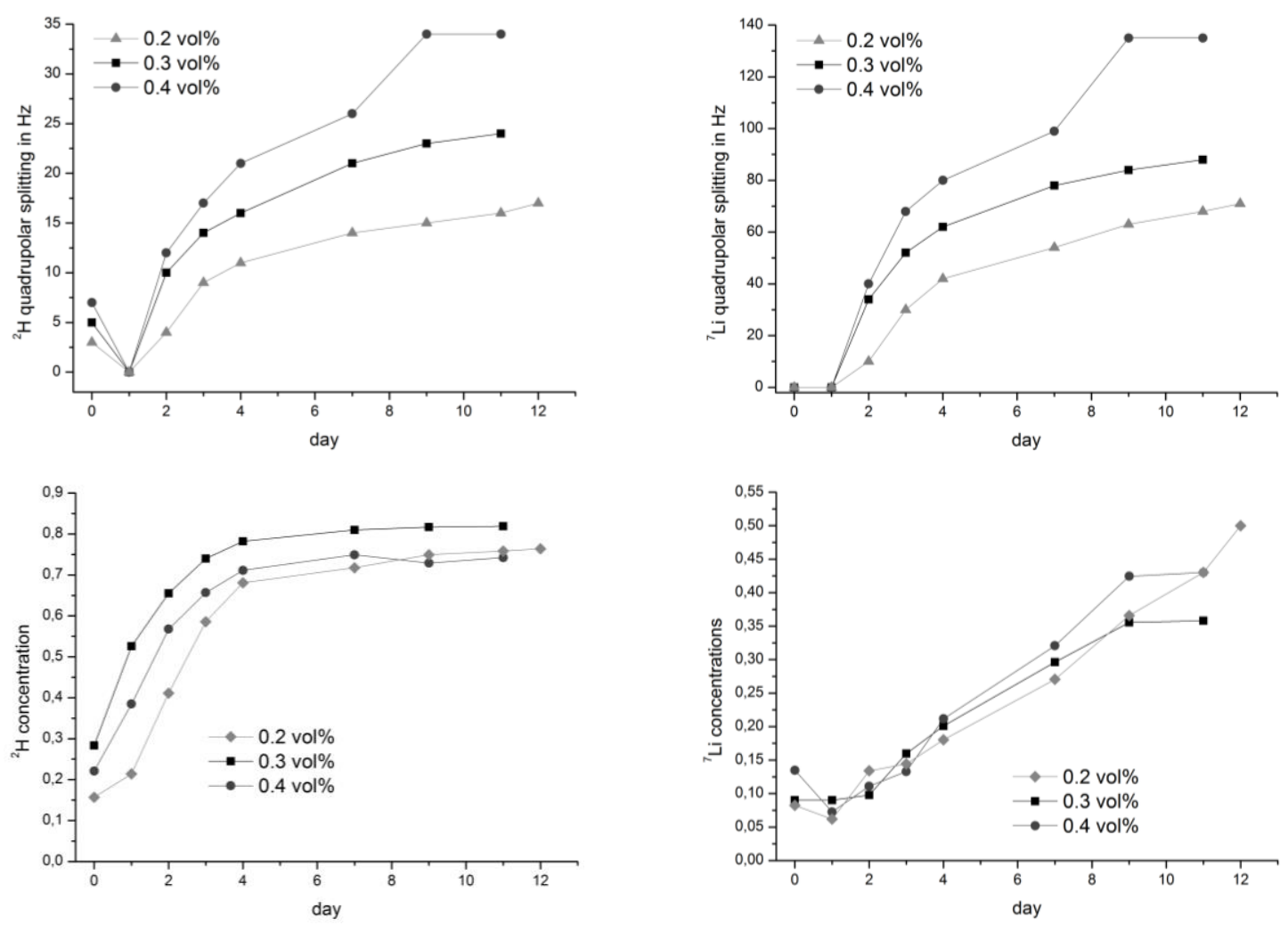

Figure 2.36: Build-up of quadrupolar splittings and concentrations in the central $(z=0)$ slice for different linker concentrations. Methyl- ${ }^{2} \mathrm{H}$ quadrupolar splitting of toluene- $d_{8}$ (top left), ${ }^{7} \mathrm{Li}$ quadrupolar splitting of 4 (top right), concentration of toluene- $d_{8}$ (bottom left) and concentration of 4 (bottom right).

The diffusion would also be expected to depend on the degree of cross-linking, ${ }^{[11,12]}$ but surprisingly, all three samples showed virtually identical swelling times as estimated from the build-up of toluene- $d_{8}$ in the central $(z=0)$ slice (Figure 2.36, bottom left). Concordantly, the ${ }^{7} \mathrm{Li}$ concentration builds up at the same rate in all samples ruling out a substantial dependency of the diffusion velocity on the cross-linker concentration between 0.2 and 0.4 vol\%.

\subsubsection{Dependency of the Swelling on the Temperature}

Since many highly sensitive lithium compounds can only be handled at low temperatures, the temperature dependency of polymer swelling is naturally an important issue for the application of strain induced alignment in a gel (SAG) to organometallic systems. For this purpose, four 0.3 vol\% cross-linked PS samples with 4 in toluene- $d_{8}$ were prepared and measured under identical conditions, but meanwhile stored at different temperatures. In all cases the gel appeared optically homogeneous after eleven days. In general, evolution of both the ${ }^{2} \mathrm{H}$ and ${ }^{7} \mathrm{Li}$ quadrupolar splittings show a clear trend for swelling temperatures of 40, 22 and $4{ }^{\circ} \mathrm{C}$ (Figure 2.37, top): decreasing the temperature leads to a slower build-up. However, the ${ }^{2} \mathrm{H}$ quadrupolar splitting at a swelling temperature of $-20{ }^{\circ} \mathrm{C}$ builds up 
substantially slower and in the case of ${ }^{7} \mathrm{Li}$ is scarcely present in the investigated time interval.
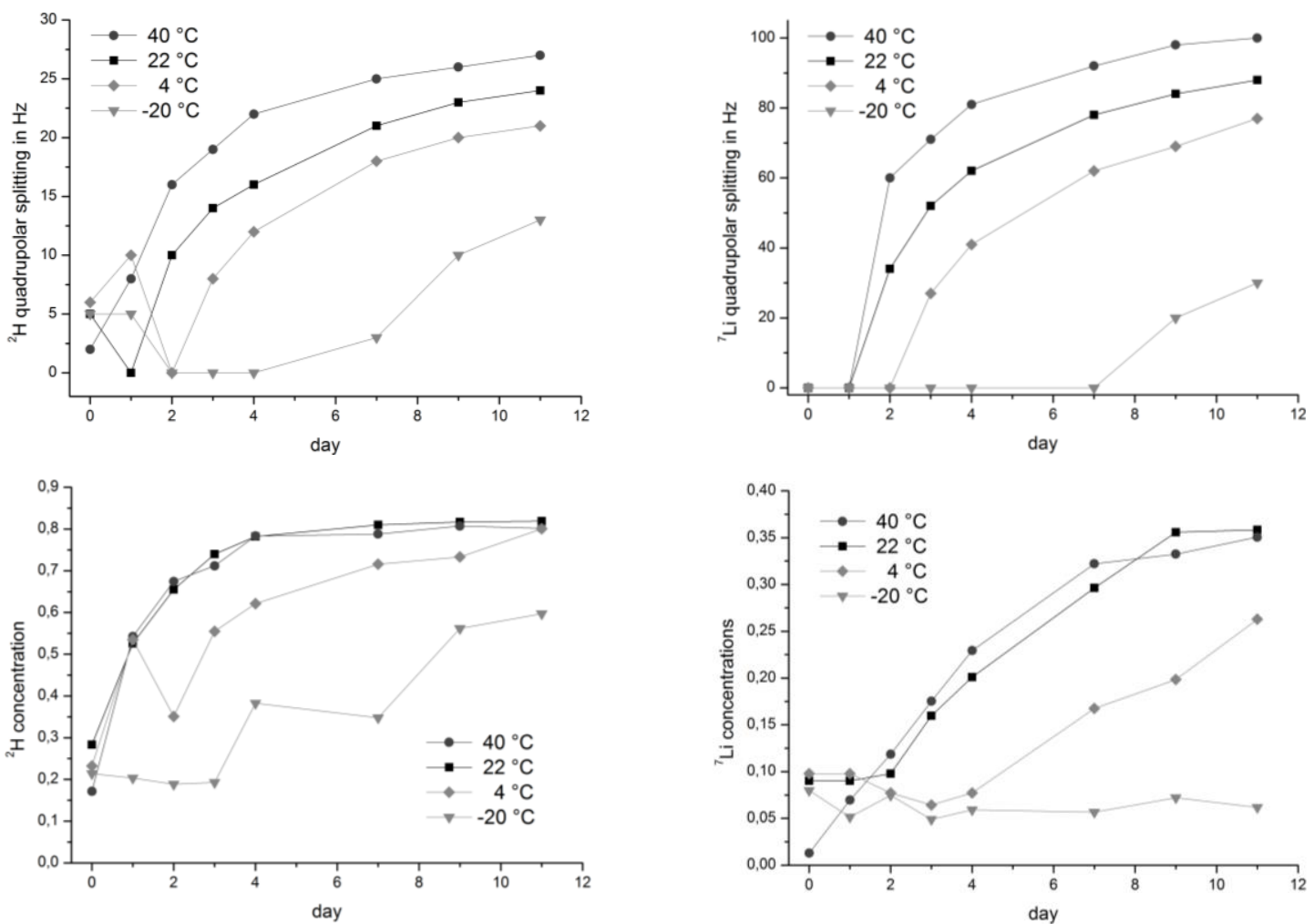

Figure 2.37: Build-up of quadrupolar splitting and concentrations in the central $(z=0)$ slice for different swelling temperatures $\left(+40,+22,+4,-20{ }^{\circ} \mathrm{C}\right)$. Methyl $-{ }^{2} \mathrm{H}$ quadrupolar splitting of toluene- $d_{8}$ (top left), ${ }^{7} \mathrm{Li}$ quadrupolar splitting of 4 (top right), concentration of toluene- $d_{8}$ (bottom left) and concentration of 4 (bottom right).

The build-up of solvent and solute concentrations, again measured in the central $(z=0)$ slice is depicted in the bottom of Figure 2.37. While the build-up of toluene- $d_{8}$ is identical at +40 and $+22{ }^{\circ} \mathrm{C}$, it occurs significantly slower at lower temperatures, which is in agreement with earlier reports. ${ }^{[11,13]}$ Compared to the ${ }^{2} \mathrm{H}$-concentration, build-up of $\mathbf{4}$ was generally slower irrespective of the swelling temperature and no build-up at all was measurable at $-20^{\circ} \mathrm{C}$. This permits to conclude that flexible temperature handling between +40 and $+4{ }^{\circ} \mathrm{C}$ is easily conceivable, but adjustment to lower temperatures needs further improvement of the polymeric material to facilitate a reasonable diffusion time, while preserving the high stability of the polymer environment towards organometallic compounds.

\subsubsection{Connecting Remarks}

Although the general idea to measure concentration profiles of solvents diffusing into a polymer is not new, ${ }^{[175]}$ this approach additionally provides quadrupolar splitting profiles that represent the induced strain as a function of the position inside the polymer gel. As 
could be shown above much useful information with respect to swelling and the polymer network is made accessible by this method.

Concerning low-temperature handling techniques, it could be verified that not only measurements but also swelling and therefore storing of the sample is feasible at moderately low temperatures (around $0{ }^{\circ} \mathrm{C}$ ), which might significantly improve the analysis of unstable, highly reactive organometallic compounds.

In terms of anisotropic NMR, the profiles could shed light on many different aspects of the SAG method. First, the possibility to map the axial strain was used in this work to confirmed that the strain is generally largest in the outer parts of the gel, whereas in the gel core it builds up slower. Resuming these observations, the local strain appears to depend on many forces that interplay in a complex fashion. Another aspect is that single slices display significantly smaller line widths while the corresponding uniformly excited spectra result in broad lines due to the heterogeneity of the sample along the field axis. Therefore, accomplishing slice-selective NMR experiments might facilitate the interpretation of otherwise heavily overlapping signals which in the case of lithium hexamethyldisilazane (4) in THF- $d_{8}$ were impeding a straightforward analysis of the dimer-monomer equilibrium (chapter 2.2.1.2). The immense improvement in terms of resolution is illustratively depicted in Figure 2.38 in an overlay of the uniform ${ }^{7} \mathrm{Li}$ and slice-selective ${ }^{7} \mathrm{Li}$ NMR spectrum of the middle slice, where the two overlapping triplets are

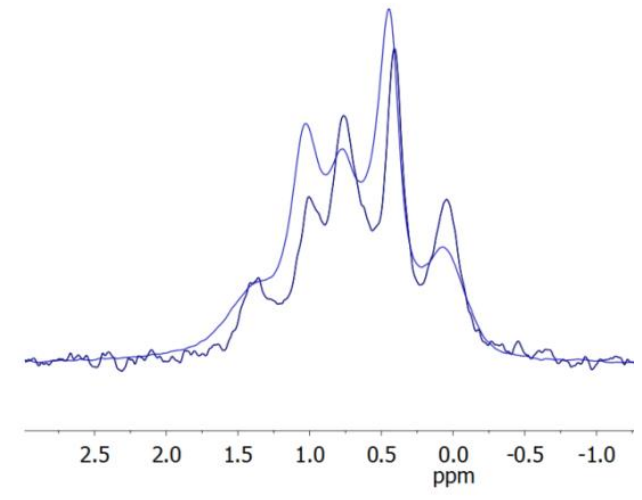
clearly visible and RQCs can be extracted without the need for further spectrum simulation. Furthermore, the SSE method allows assessment of the sample homogeneity prior to measurements of anisotropic NMR parameters within a few minutes on standard NMR probes. This might be beneficial as despite the optical homogeneity, the gel inside the volume detected by the RF coil was not uniformly strained even after 12 days of swelling thus causing reduced resolution of extracted anisotropic parameters, e.g. RDCs. Furthermore, heterogeneities like breaks in the gel are straightforwardly located. Sliceselective excitation (SSE) might also significantly alleviate the problem of sample heterogeneity because SSE may be incorporated into more complex $1 \mathrm{D}$ and 2D NMR experiments that have been established for the measurement of RDCs, RCSAs and RQCs. 
Thereby, smaller line widths and thus a higher accuracy might be accomplished for the determination of anisotropic parameters.

By selecting a slice located within the freshly swollen polymer, quadrupolar splittings are qualitatively visible shortly after sample preparation, whereas uniform excitation on the same sample does not yield a clear (albeit poorly resolved) ${ }^{2} \mathrm{H}$ quadrupolar doublet before two days, and a ${ }^{7} \mathrm{Li}$ quadrupolar triplet before four days. Consequently, for a rough evaluation of anisotropic parameters, the overall time required for sample preparation is reduced from more than a week to merely a few days of swelling.

Likewise, polymer scientists may substantially profit from the SSE technique enabling them to get better insight into their synthesized network structures. Time- and positiondependent concentration profiles allow a thorough investigation of the distinct assemblies inside a polymeric system and address sample homogeneity as well as entanglement and swelling capacities.

How the employment of slice-selective NMR experiments can improve the analysis of polymer networks and how essential details can be deduced for the subsequent modification and improvement of polymer networks for SAG will be discussed in the following chapter. 


\subsubsection{Investigations of Different Polymer Systems: PBA and Modification of PS}

In the following, the methods introduced for a PS / toluene system will be further applied to butyl (and ethyl) acrylate polymer systems. In particular, butyl acrylate (BA) gels were promoted by the broad scope of different solvents that form strained gels with PBA. This might be an interesting alternative to other polymer gels although the ester moiety of the acrylate group undoubtedly prevents the application of BA to highly reactive compounds, for instance organolithiums (Figure 2.39). Furthermore, the swelling of cross-linked PBA is substantially faster than that of polystyrene which could be beneficial for the investigation of general network and homogeneity phenomena. Hence, conclusions can be drawn from these straightforward characterizations and transferred to the slowly swelling yet inert PS systems. This chapter will be

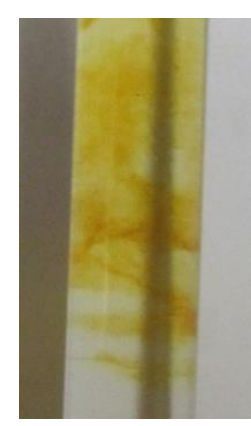

Figure 2.39: LiHMDS 4 in PBA / toluene- $d_{8}$. concerned with the establishment of thermally polymerized BA gels as possible new polymer network to partially align a variety of compounds for anisotropic NMR measurements. Subsequently, UV-initiated polymerization of BA will be compared to networks of PBA obtained by reversible addition fragmentation chain transfer (RAFT) polymerization. By means of slice selective NMR experiments the sample homogeneity and network structure of the gel is addressed. The last part will focus on the transfer of the discussed results to polystyrene networks.

\subsubsection{Thermally Polymerized Butyl (and Ethyl) Acrylates}

Polybutyl and polyethyl acrylates with the bifunctional cross-linker 1,4-butanediol diacrylate (BDDA) (Figure 2.40, right) were prepared according to the procedure illustrated earlier for cross-linked polystyrene networks. Butyl acrylates with 0.6 vol\% BDDA and ethyl acrylates with $0.4 \mathrm{vol} \%$, respectively, were polymerized thermally at $115^{\circ} \mathrm{C}$ inside sealed glass tubes with an inner diameter of $3.8 \mathrm{~mm}$. Lower polymerization temperatures and lower cross-linker concentrations (e.g. 0.3 vol\%) yielded inferior polymeric material and were therefore discarded. After two days at elevated temperatures, the glass tubes were broken and homogeneous parts of the polymer cut into pieces of $1 \mathrm{~cm}$ length. Interestingly, the material properties of these sticks significantly differ from the appearance of polystyrene. While polystyrene sticks obtained from bulk polymerization are very rigid, almost glassy and robust, PBA and PEA networks already display jelly-like behaviour in the non-swollen state and additionally can be easily folded or torn into pieces (Figure 2.40, left). This can be explained by the different glass temperatures 
$\mathrm{T}_{\mathrm{g}}(\mathrm{PS})=110^{\circ} \mathrm{C}$ and $\mathrm{T}_{\mathrm{g}}(\mathrm{PBA})=-55^{\circ} \mathrm{C}$ below which the polymer shows glass-like properties. ${ }^{[176]}$ Although these values are strictly only valid for linear polymers, the difference between the two values is big enough to neglect effects induced by the network structure.
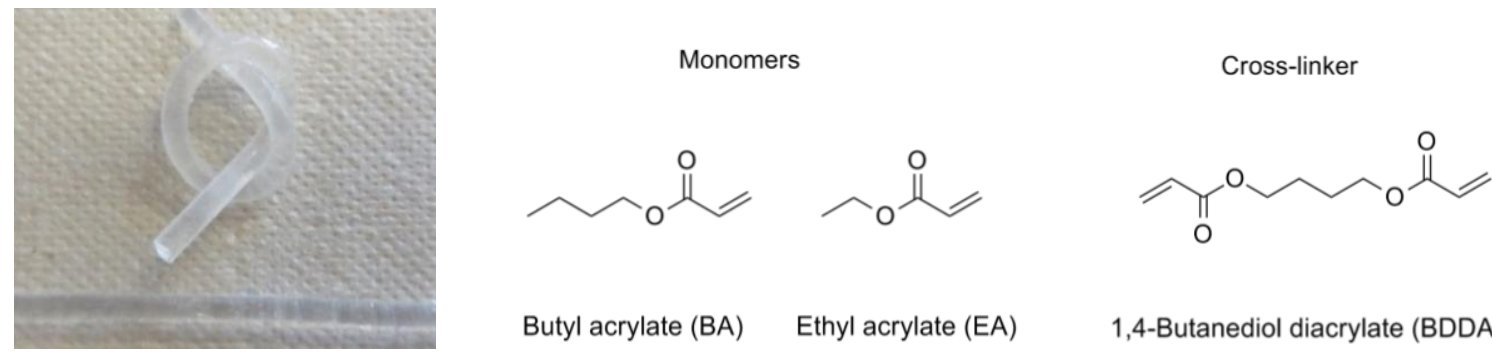

Butyl acrylate (BA) Ethyl acrylate (EA)

1,4-Butanediol diacrylate (BDDA)

Figure 2.40: Photograph of thermally polymerized butyl acrylate sticks (left) and representation of the employed monomers (BA, EA) and cross-linker (BDDA) (right).

The sticks were then transferred into standard NMR sample tubes and swelling was monitored over time for several solvents including THF- $d_{8}$, methanol- $d_{4}$, acetone- $d_{6}$, toluene- $d_{8}$, DMSO- $d_{6}$ and acetonitrile- $d_{4}$. With the exception of acetonitrile, all solvents resulted in a homogeneously swollen polymer gel certifying polybutyl acrylate networks a broad scope of solvents. Swelling of the PBA sticks was complete after three days. ${ }^{2} \mathrm{H}$ NMR measurements were carried out to verify partial alignment for the above-mentioned solvents inside the polybutyl acrylate environment with defined quadrupolar splittings. The overall quadrupolar splitting is considerably small in case of PBA indicating the presence of larger cavities that is to say, longer polymer chains between the cross-links (junction point), and therefore a smaller degree of alignment. For example, comparison of PBA with PS gels, both with a cross-linker concentration of 0.6 vol\% and swollen in THF- $d_{8}$, reveals a significant decrease of the ${ }^{2} \mathrm{H}$ quadrupolar splitting (PS: $\sim 80 \mathrm{~Hz}$ vs. PBA: $\sim 10 \mathrm{~Hz}$ for the $\mathrm{CH}_{2}$-group resonating at $3.58 \mathrm{ppm}$ ) at equilibrium swelling. This may be due to the increased length of BDDA (over DVB as linker), further separating the junction points and leading to larger cavities which could also partially account for the observed enhanced swelling speed. Additionally, both polymerizations were carried out at high temperatures and the polymerizing vinyl moieties only vary in the attached side chain $(\mathrm{R}=\mathrm{Ph}$ or $\mathrm{Bu})$. For butyl side chains an alternative mechanism which is referred to as intramolecular transfer to polymer or backbiting (Scheme 2.6) is also important. 


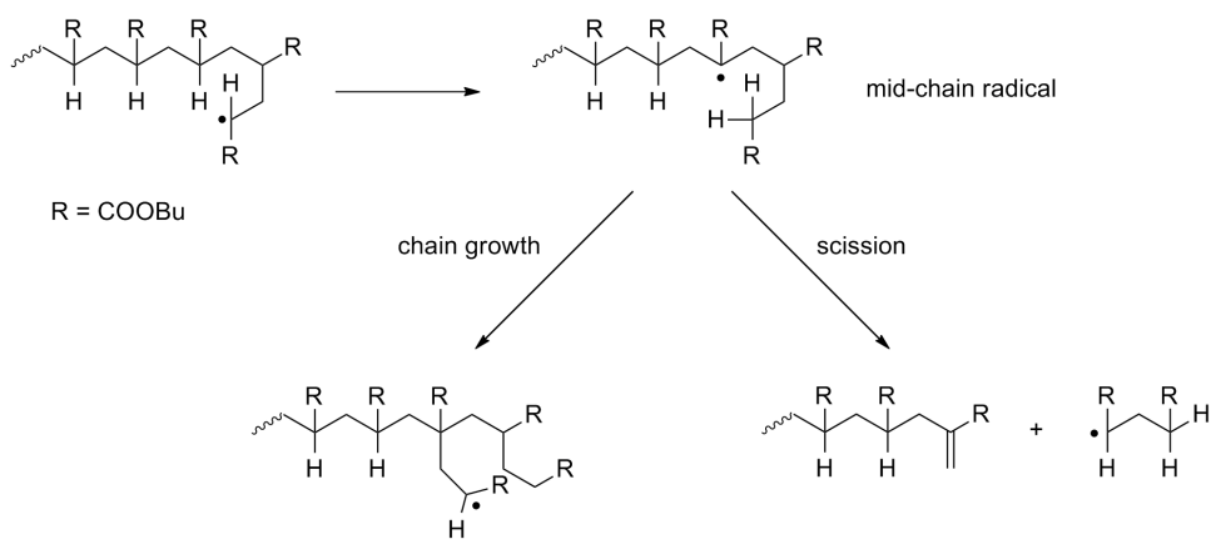

Scheme 2.6: Overview of side reactions known for butyl acrylate polymerizations at elevated temperatures: intramolecular $\mathrm{H}$-abstraction ("backbiting") leads to the formation of mid-chain radicals which in turn can add monomers or undergo $\beta$-scission resulting in a macro-monomer and a new short-chain radical. ${ }^{[177]}$

Intramolecular transfer to polymer, where the propagating chain-end radical folds back and abstracts a hydrogen atom from an acrylate unit on its own backbone via a sixmembered ring-like transition state, has been well established in high-temperature ethylene homopolymerization along with ethylene/ $n \mathrm{BA}$ copolymerizations. ${ }^{[178]}$ The resulting tertiary mid-chain radicals can subsequently add monomers (slow chain growth) or undergo fragmentation into a macro-monomer and a new short-chain radical ( $\beta$-scission). A critical evaluation of the kinetics of $n \mathrm{BA}$ free-radical polymerizations as well as the branching caused by these secondary reactions were thoroughly investigated by many research groups. ${ }^{[177,179]}$ Recently, Buback et al. were able to provide kinetic data and mechanistic details including the above-presented backbiting mechanism by pulsed laser polymerization experiments. ${ }^{[180]}$ As EPR measurements proved these side reactions to gain importance with increasing temperature they have to be definitely taken into account for the thermally initiated polymerization procedure applied herein. ${ }^{[181]}$ The secondary reactions and the incidence of backbone radicals impede the controlled construction of a regular cross-linked network which could account for the looser networks observed for thermally polymerized PBA. ${ }^{[182]}$ Due to the different molecular structure, no backbiting is observed in the polymerization of styrene.

Another interesting difference between PS and PBA is encountered in the orientation of the corresponding deuterated solvent molecules becoming most evident for toluene- $d_{8}$ (Figure 2.41). While in case of PS the partial orientation of the toluene molecules leads to a smaller quadrupolar splitting of the para deuterium atoms (inner doublet) compared to the ortho and meta positions (outer doublets), the situation is reversed for molecules inside PBA polymer gels. 


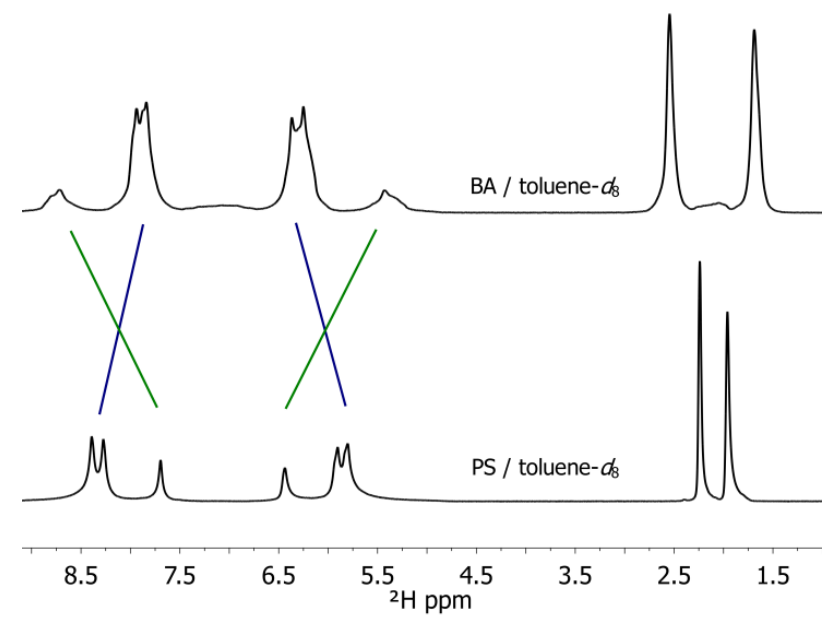

Figure 2.41: ${ }^{2} \mathrm{H}$ NMR spectra of PBA (0.6 vol\% BDDA)/toluene- $d_{8}$ and PS ( 0.2 vol\% DVB)/ toluene- $d_{8}$ illustrating reversed quadrupolar splitting for the aromatic ortho, meta and para deuterium atoms.

The nature of the magnitude of the side groups (aromatic $\pi$-system vs. alkyl chain) and their polarity (non-polar vs. ester moieties) might give an explanation for the different orienting properties of the two gel environments. An advantage of PBA polymer gels is the superior low temperature performance. ${ }^{2} \mathrm{H}$ NMR measurements of PBA / acetone- $d_{6}$ gels allowed an observation of quadrupolar splittings down to $-90^{\circ} \mathrm{C}$ (Figure 2.42) thus facilitating the analysis of highly dynamic systems. Additionally, more structural information might be obtained by measuring a second set of anisotropic NMR parameters in a different alignment medium.

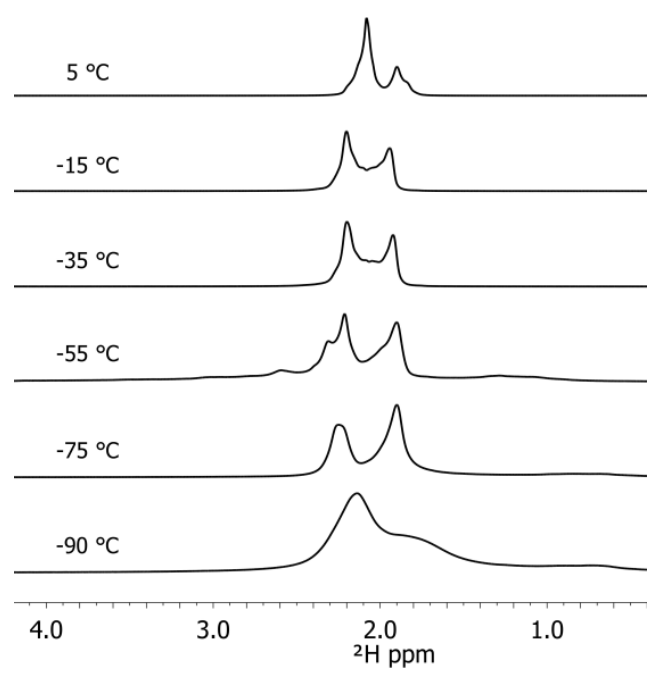

Figure 2.42: Low temperature ${ }^{2} \mathrm{H}$ NMR experiments of BA ( 0.6 vol\% BDDA) / acetone- $d_{6}$ confirming the applicability of this type of gels down to nearly $-90^{\circ} \mathrm{C}$, which may be advantageous for the analysis of flexible molecules.

This was also verified for PBA polymer sticks swollen in toluene- $d_{8}$, which support temperatures of $-60^{\circ} \mathrm{C}$ and lower. 


\subsubsection{Homogeneity of Different Butyl Acrylate Systems}

All measurements of anisotropic NMR parameters critically depend on the stability and homogeneity of the alignment medium. Since "highly nonuniform spatial distributions of polymer network concentration and cross-linking density" ${ }^{[183]}$ are attributed to cross-linked polymers obtained by free radical polymerization, reversible-deactivation radical polymerization techniques are nowadays practised to yield materials characterized by more uniform molecular weights and an overall increased homogeneity. Important representatives of these reversible-deactivation techniques are nitroxide-mediated polymerization (NMP), ${ }^{[184]}$ atom transfer radical polymerization (ATRP) ${ }^{[185]}$ and reversible addition-fragmentation chain transfer $(\mathrm{RAFT})^{[186]}$ polymerization. In all of these methods, equilibrium reactions are introduced by the addition of controlling agents that are able to capture and release free radicals. In this work, RAFT polymerized networks were analysed and the corresponding underlying mechanisms will be further explained in the following (Scheme 2.7): ${ }^{[187]}$

(l)

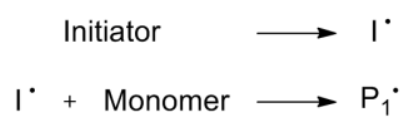

(II)

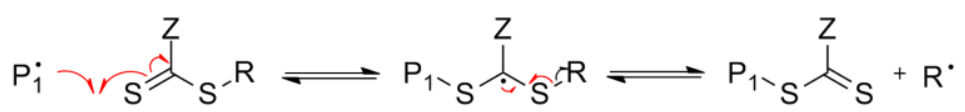

(III)

$$
\begin{aligned}
& \mathrm{P}_{\mathrm{n}}^{\cdot}+\text { Monomer } \longrightarrow \mathrm{P}_{\mathrm{n}+1}^{\cdot} \\
& \mathrm{R}^{\cdot}+\text { Monomer } \longrightarrow \mathrm{P}_{1}^{\cdot}
\end{aligned}
$$

(IV)

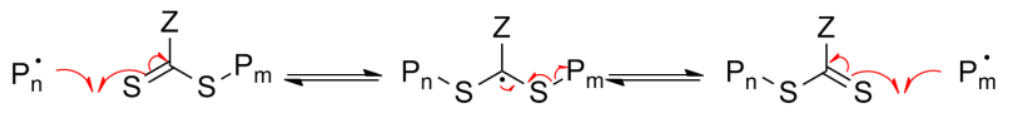

(V)

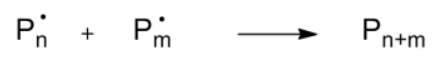

Scheme 2.7: Illustration of the general reaction steps proceeding in a reversible addition fragmentation chain transfer (RAFT) polymerization with $\mathrm{Z}$ being a stabilizing group (e.g. Ph, SR, OR) and $\mathrm{R}$ an organic leaving group.

Starting from the general mechanism of free radical polymerization consisting of (I) initiation, (III) propagation and (IV) termination steps, two equilibria (II + IV) are encountered due to the presence of a RAFT agent (usually containing a dithio- or trithiomoiety). In the first equilibrium (II), the propagating chain $\left(\mathrm{P}_{1}{ }^{-}\right)$reacts with the RAFT molecule yielding the so-called "dormant" species, which releases the radical R' from the intermediate by homolytical cleavage of the $S-R$ bond. The resulting radical $R \cdot$ can add 
monomers in the same way as the propagating chain (III). The second equilibrium step (IV) is the key of the RAFT process, because the addition and fragmentation of propagating chain macroradicals with another dormant species allows the molecular weight to increase linearly with conversion and thus to obtain an overall narrow molecular weight distribution. The additional fast equilibria during the RAFT mechanism should also produce less heterogeneous materials compared to the free radical polymerization.

An increased homogeneity of the polymer network has been recently investigated on the basis of different experimental techniques ranging from UV-spectroscopy, ${ }^{[188]}$ dynamic light scattering ${ }^{[189]}$ and analysis of the shrinking kinetics ${ }^{[190]}$ and can be explained as schematically depicted in Figure 2.43.

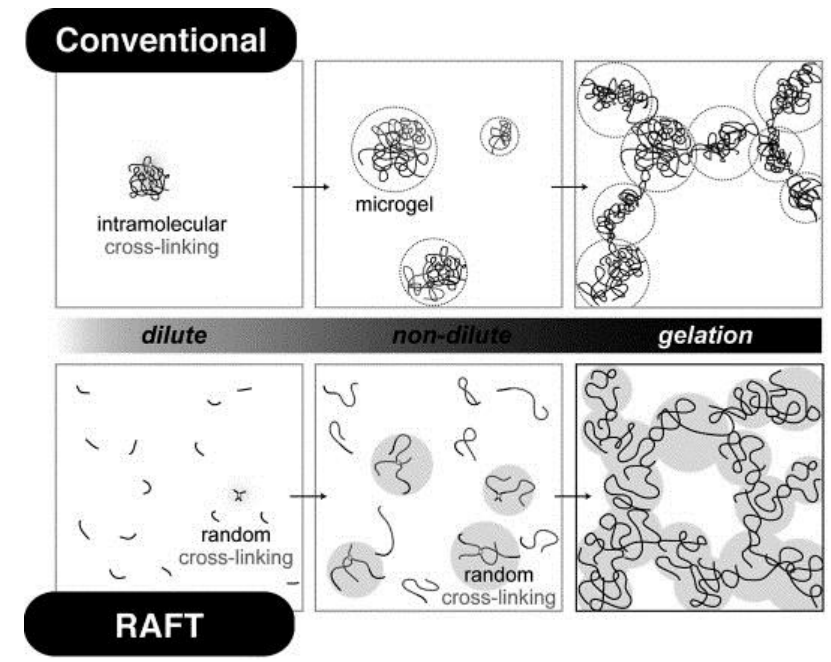

Figure 2.43: Schematic overview of the intrinsic structure of gels during different stages of conventional and RAFT polymerizations. ${ }^{[189]}$

A comparing of the cross-linking and gelation behaviour of conventional, free radical systems with RAFT polymerizations shows that at an early stage of the reaction intramolecular cross-linking dominates for the former and random cross-linking is observed for the latter. For the very fast occurring conventional free radical polymerization this leads to unexpanded primary chains in the course of the reaction which will be more frequently cross-linked intramolecularly resulting in an attachment of closely packed coils ("microgels"). In contrast, random cross-linking is continuously observed for RAFT polymerizations and the slower chain growth due to the two RAFT equilibria (retardation effect in thioesther-mediated polymerizations ${ }^{[191]}$ allows the chains to relax which will favour intermolecular cross-linking so that an overall hyperbranched, homogeneous structure with a higher amount of dangling chains is finally obtained. Microgel formation is not observed at any stage of the RAFT polymerization. 
In the following, it will be discussed in which way slice-selective NMR experiments may contribute to the analysis of the above-mentioned homogeneity issues of differently polymerized PBA networks. The work was carried out in cooperation with Rouven Henkel from the research group of Prof. Vana. According to Table 2.2, several PBA polymers were prepared by UV-initiated polymerization of butyl acrylate in the presence of BDDA as cross-linker and s-ethyl-propane-2-ylonate-s'-hexyl-trithiocarbonate (EPHT) as chain transfer agent (CTA, RAFT agent).

Table 2.2: Summary of polymer compositions used in this work. The RAFT agent utilized was EPHT.

\begin{tabular}{cccc}
\hline Monomer & Linker & mol\% Linker & mol\%o RAFT \\
\hline BA & BDDA & 0.5 & 0.0 \\
BA & BDDA & 0.5 & 1.0 \\
BA & BDDA & 0.5 & 2.0 \\
BA & BDDA & 1.0 & 0.0 \\
BA & BDDA & 1.0 & 2.0 \\
\hline
\end{tabular}

The amount of RAFT agent necessary for inducing a reversible-deactivation radical polymerization was very low (0-2 mol\%o). Furthermore, swelling experiments of the PBA polymers in acetone were carried out by Rouven Henkel in which the following dependencies of the volume swelling degree were observed:

(1) with increasing amount of the RAFT agent, the volume swelling degree increased whereas

(2) with a constant amount of RAFT agent, the volume swelling degree increases with decreasing cross-linker concentration.

In general, all polymers obtained in the presence of the RAFT agent showed a higher degree of swelling. Furthermore, mechanical investigations attribute a higher elasticity to the BA polymers derived from reversible-deactivation radical polymerization, especially for linker concentrations of $1 \mathrm{~mol} \%$ or below. 


\subsubsection{Slice-selective Experiments}

According to the procedure described in chapter 2.2.2, PBA sticks $(\phi 3.8 \mathrm{~mm}$, length $1 \mathrm{~cm}$ ) were prepared and sliceselective ${ }^{2} \mathrm{H}$ isotropic reference measurements of pure acetone- $d_{6}$ and toluene- $d_{8}$ were carried out. The sticks were adjusted to the centre of the coil region. In order to be able to introduce the soft and sticky BA / RAFT sticks into the NMR sample tube, they had to be manipulated by cutting out a

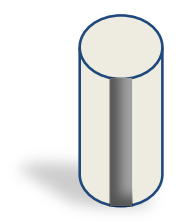

Figure 2.44: Cutting position for the PBA sticks. small $(0.1 \mathrm{~mm})$ part as depicted in Figure 2.44.

\subsection{General Observations}

The BA sticks were put to swell in $0.5 \mathrm{~mL}$ acetone- $d_{6}$ and toluene- $d_{8}$, respectively, and the swelling was monitored for 24 hours by performing slice-selective ${ }^{2} \mathrm{H}$ measurements $0,3,6$, 12 and $24 \mathrm{~h}$ after sample preparation. Concentrations of linker and RAFT agent were varied as stated in Table 2.2. Analogous to the analysis of polystyrene gels by slice selective NMR measurements, the solvent concentrations and ${ }^{2} \mathrm{H}$ quadrupolar splittings are extracted for each slice yielding spatially resolved profiles for a given swelling time or timeresolved build-up curves for a given slice. Corresponding concentration and splitting profiles in acetone- $d_{6}$ are exemplarily depicted for polymer sticks with $0.5 \mathrm{~mol} \%$ crosslinker polymerized in the presence of 0 and 1 mol\%o RAFT agent (Figure 2.45). The profiles show the expected behaviour, i.e. a swelling process that commences at the outer upper and lower slices of the stick with almost no solvent in the central region. The original dimensions of the PBA sticks can be clearly identified from the plateaus found for the concentration profiles of PBA polymerized without a RAFT agent (Figure 2.45 top, slices -2 to +2 ) directly after sample preparation. At this early stage of swelling, no quadrupolar splittings are observed. Subsequently, the solvent penetrates more and more polymer slices yielding higher and more equilibrated solvent concentrations as well as quadrupolar splittings over the whole sample. 

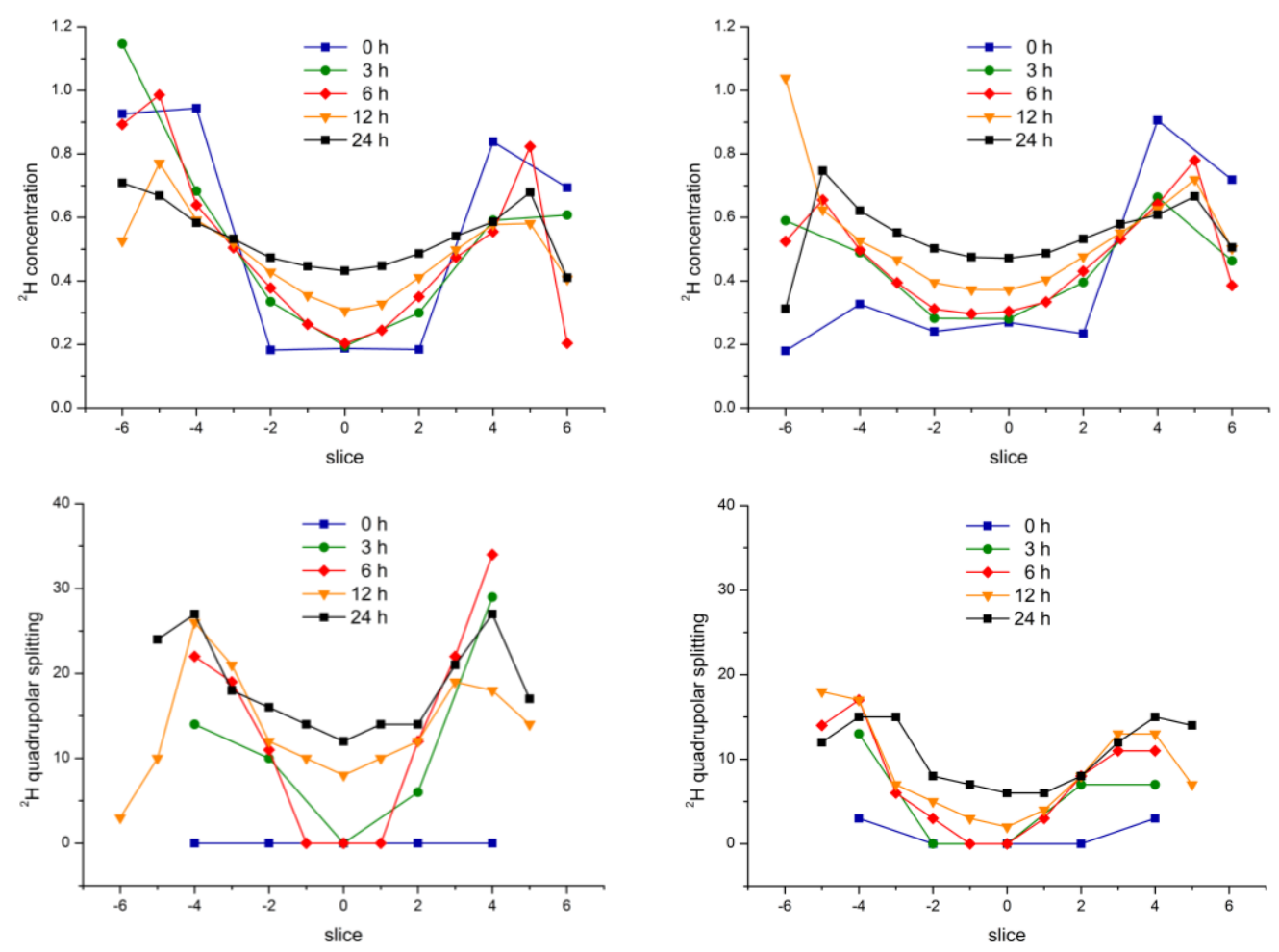

$0.5 \mathrm{~mol} \%$ BDDA, $0.0 \mathrm{~mol} \%$ RAFT

0.5 mol\% BDDA, 1.0 mol\% RAFT

Figure 2.45: Comparison of the concentration (top) and quadrupolar splitting (bottom) profiles of PBA / acetone- $d_{6}$ gels with $0.5 \mathrm{~mol} \% \mathrm{BDDA}$ and varying RAFT concentration $(0-1 \mathrm{~mol} \%)$.

After a swelling time of only 24 hours the system is significantly equilibrated. When comparing the solvent concentration profiles in the absence (top left) and in the presence (top right) of the RAFT agent no significant difference is observed with similar endconcentrations for both polymer materials yet the polymeric material obtained in the presence of the RAFT agent seems to reach homogeneity faster. However, the size of the ${ }^{2} \mathrm{H}$ quadrupolar splitting varies significantly between the PBA stick polymerized under freeradical conditions (bottom left) and the one polymerized in the presence of the RAFT agent (bottom right). The latter polymer yields almost halved values, which may be attributed to an expanded pore size of the RAFT polymers. Since the solvent concentrations are independent of the RAFT agent, the number of pores per volume (i.e. the relative density of the polymer) then has to decrease. Similar behaviour was found for the other polymer compositions swollen in acetone- $d_{6}$ as will be discussed below.

In the following, concentration and splitting profiles for the same polymers but swollen in toluene- $d_{8}$ are discussed (Figure 2.46). 

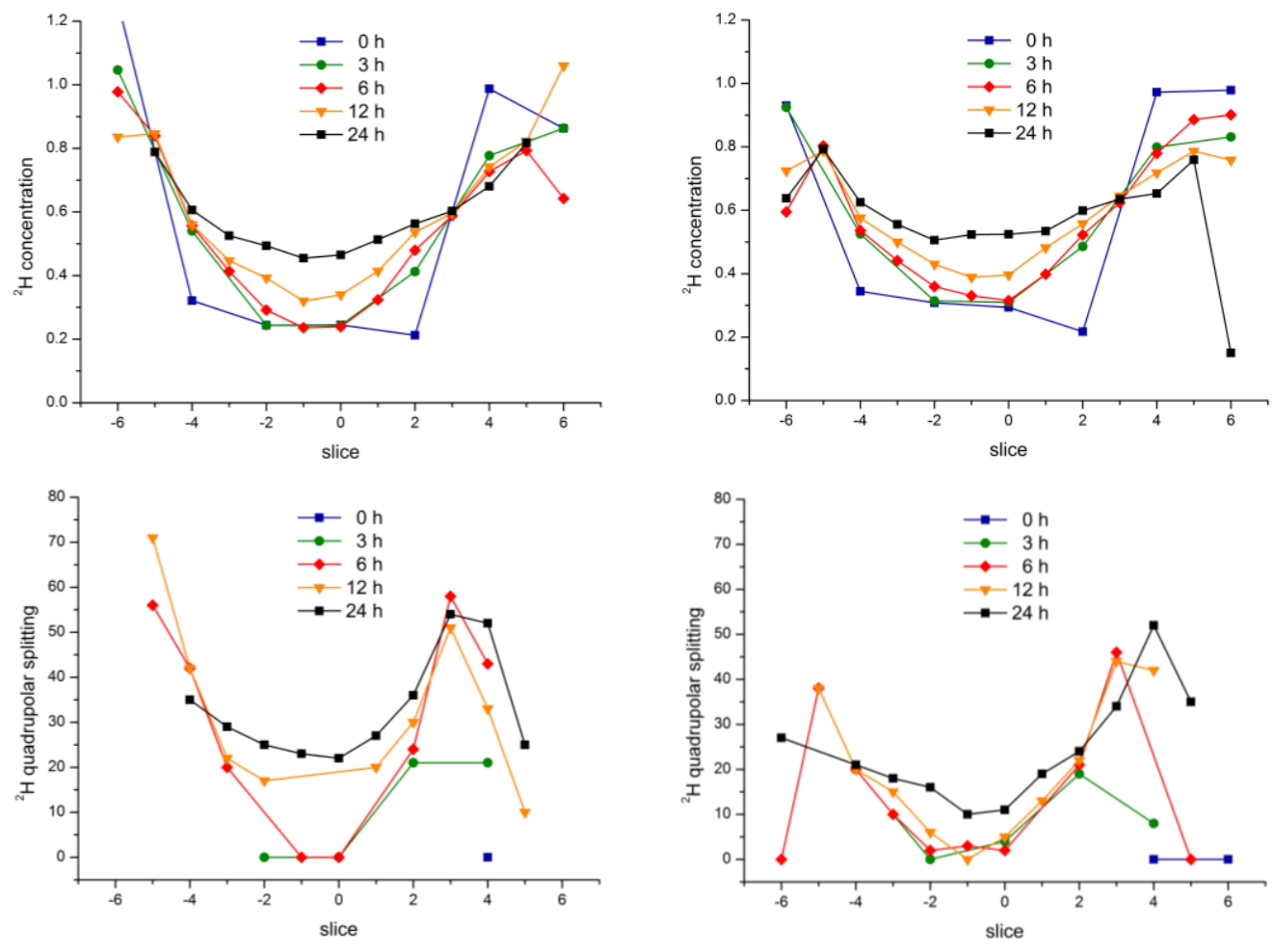

0.5 mol\% BDDA, 0.0 mol\%o RAFT

$0.5 \mathrm{~mol} \%$ BDDA, 1.0 mol\%o RAFT

Figure 2.46: Comparison of the concentration (top) and quadrupolar splitting (bottom) profiles of PBA / toluene- $d_{8}$ gels with $0.5 \mathrm{~mol} \% \mathrm{BDDA}$ and varying RAFT concentration $(0-1 \mathrm{~mol} \%)$.

Only the methyl group of toluene was taken into account due to the above-mentioned smaller phase distortion compared to the aromatic signals. The overall trend is analogous to the swelling series performed with acetone- $d_{6}$, and a reduction of the degree of alignment in the presence of the RAFT agents is likewise observed for these PBA / toluene$d_{8}$ gels. Both solvents reach an average penetration of $50-60 \%$ after $24 \mathrm{~h}$. A possible advantage of the PBA/toluene- $d_{8}$ system are the larger splitting values which might facilitate the determination of small splittings at an early swelling stage by exhibiting a better peak separation. The solvent concentration inside the distinct samples seems to vary only slightly although larger cavities are expected to entail a larger solvent uptake.

\subsection{Dependency on the Linker Concentration}

To evaluate the influence of the cross-linker concentration, the build-up of the quadrupolar splitting in the middle slice $(z=0)$ was compared for representative polymer compositions both swollen in acetone- $d_{6}$ and in toluene- $d_{8}$ over the full monitored time interval $(24 \mathrm{~h})$. Small splittings observed at early stages of the swelling experiment again account for the fact that the polybutyl acrylate sticks do not flush exactly with the walls of the NMR sample tube. Expansion in the horizontal direction is thus observed until around 
$6 \mathrm{~h}$ after sample preparation. The swelling behaviour in acetone- $d_{6}$ is depicted in Figure 2.47 .
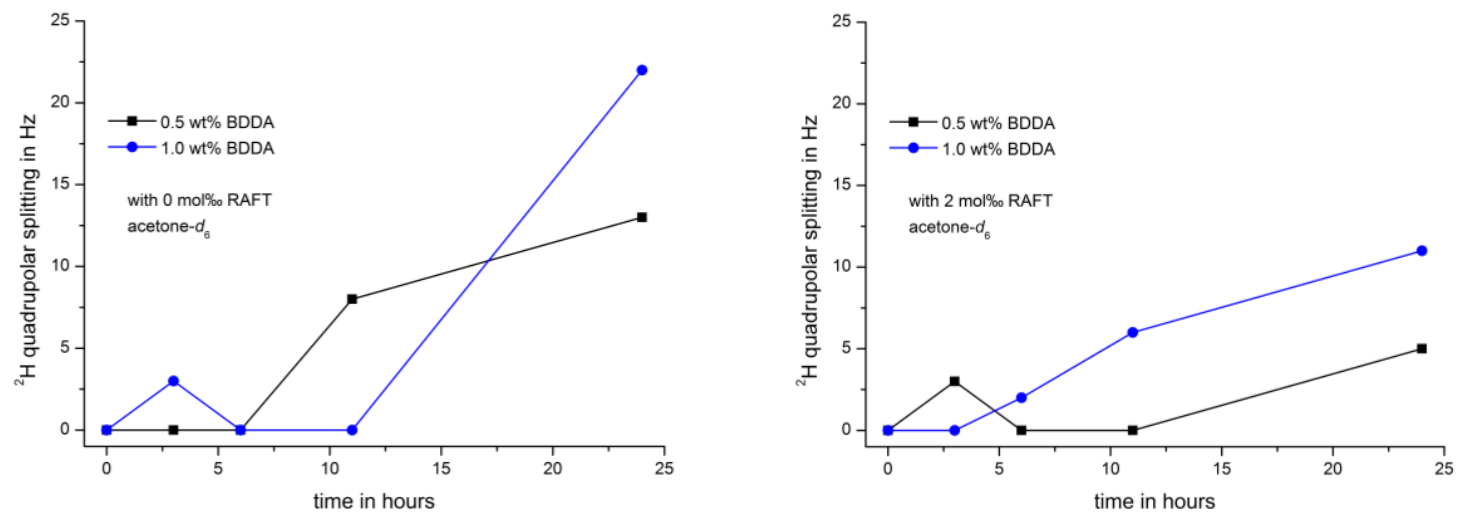

Figure 2.47: Build-up of the ${ }^{2} \mathrm{H}$ quadrupolar splitting of acetone- $d_{6}$ in the middle slice for polymer sticks obtained by free radical polymerization (left) and polymerized in the presence of 2 mol\%o RAFT agent (right). In both polymers, the amount of linker was varied (0.5 and 1.0 vol\% BDDA).

After 24 hours of swelling in acetone- $d_{6}$, polymer gels without any RAFT agent show quadrupolar splitting values of 13 and $22 \mathrm{~Hz}$ for the samples with 0.5 and $1.0 \mathrm{~mol} \%$ crosslinker, respectively. Again, this shows that higher linker concentrations cause an increased number of junction point and thus smaller cavities which in turn results in a higher quadrupolar splitting (higher degree of alignment). The same monomer linker mixtures but accompanied by 2 mol\%o RAFT agent (Figure 2.47, right) yield smaller quadrupolar splitting values $(5$ and $11 \mathrm{~Hz}$ ) but follows the same trend. Hence, these illustrations clearly underline that the RAFT agents indeed interferes with and retards the cross-linking reaction rate which results in a higher amount of dangling chains and a larger cavity size. ${ }^{[190]}$ Likewise, the swelling behaviour in toluene- $d_{8}$ is depicted in Figure 2.48.
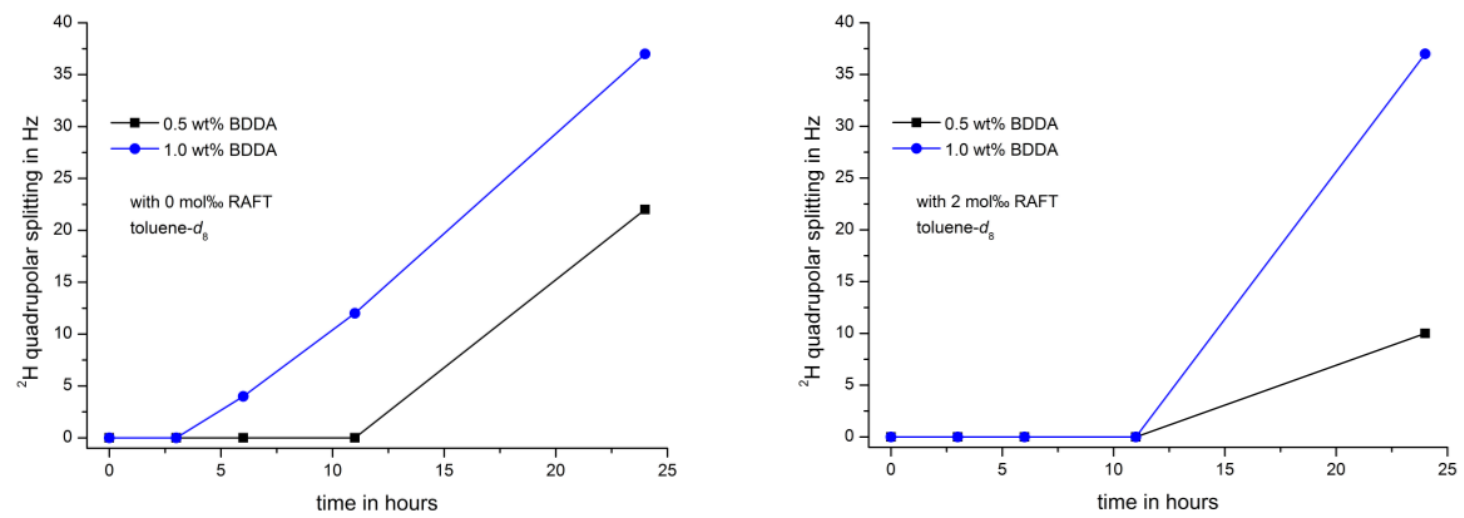

Figure 2.48: Build-up of the methyl- ${ }^{2} \mathrm{H}$ quadrupolar splitting of toluene- $d_{8}$ in the middle slice for polymer sticks obtained by free radical polymerization (left) and polymerized in the presence of 2 mol\%o RAFT agent (right). In both polymers, the amount of linker was varied (0.5 and 1.0 vol\% BDDA). 
The quadrupolar splitting of the methyl deuterium atoms in non-RAFT polymer gels follows the same trend as extracted from the acetone swelling experiments albeit at overall higher splitting values (22 and $37 \mathrm{~Hz}$ ). Again, the splitting is significantly higher for increased cross-linker concentrations. This trend is preserved despite the presence of a chain transfer agent $(10$ and $37 \mathrm{~Hz}$ ). For the RAFT polymer gels swollen in toluene an interesting behaviour was found. The $2 \mathrm{H}$ NMR signal remains a singlet up to $11 \mathrm{~h}$ after sample preparation and only afterwards shows a splitting. However, the spectra of the samples with $1 \mathrm{~mol} \% \mathrm{BDDA}$ and $2 \mathrm{~mol} \%$ chain transfer agent in toluene suffer from severe distortions probably caused by polymer defects or breaks so that these data points have to be regarded with caution. Nevertheless, the general trend derived from the acetone$d_{6}$ swelling experiments could be verified for toluene- $d_{8}$.

\subsection{Dependency on the Concentration of the RAFT agent}

In addition to the influence of the cross-linker concentration, the dependency of the RAFT concentration on the quadrupolar splitting at constant monomer cross-linker compositions was investigated. For a concentration of 0.5 mol\% BDDA, polybutyl acrylate sticks with 0,1 and 2 mol\%o chain transfer agent and swollen in acetone- $d_{6}$ were compared (Figure 2.49, left). The build-up curves nicely illustrate a decrease in the quadrupolar splitting value upon addition of the chain transfer agent prior to polymerization at all measurement times (final values: 13 to $6 \mathrm{~Hz}$ ). Interestingly, doubling the amount of RAFT agent (from 1 to 2 mol\%o) only insignificantly reduces the observed splitting value $(5 \mathrm{~Hz})$. The same trend is confirmed for polymers with a higher content of cross-linking (Figure 2.49 , right), where the splitting values are, as expected, again higher.
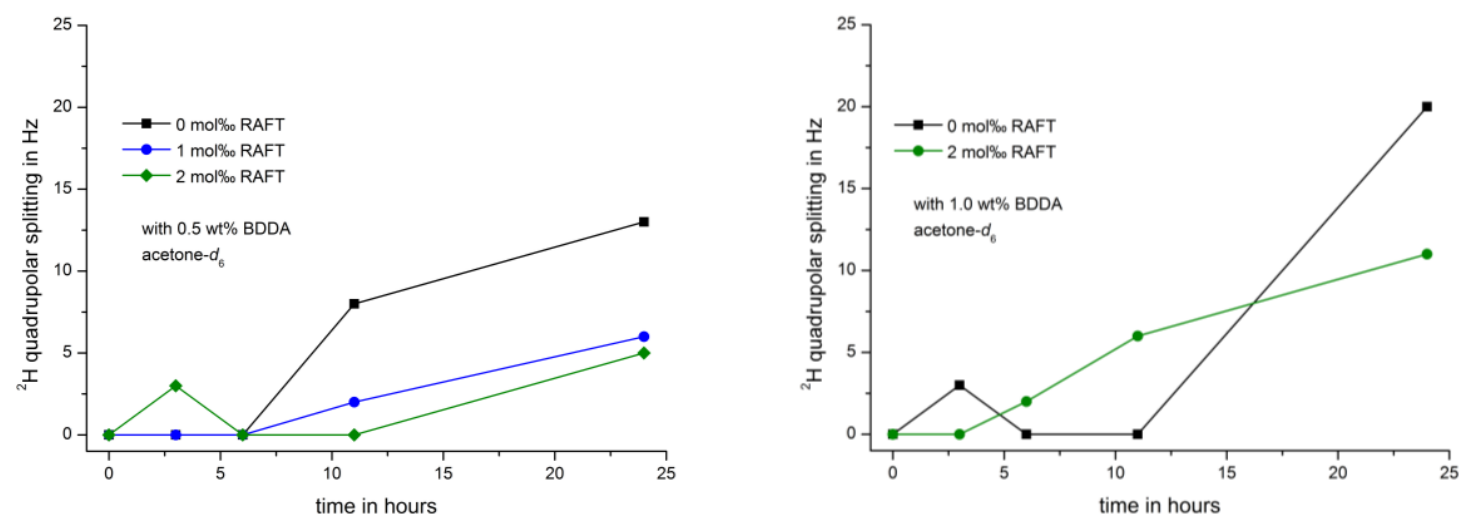

Figure 2.49: Build-up of the ${ }^{2} \mathrm{H}$ quadrupolar splitting of acetone- $d_{6}$ in the middle slice for polymer sticks with 0,1 and 2 mol\% RAFT obtained in the presence of $0.5 \mathrm{~mol} \%$ BDDA (left) and $1 \mathrm{~mol} \%$ BDDA (right). 
Performing analogous swelling experiments in toluene- $d_{8}$ again yields an overall confirmation of the above mentioned tendencies (Figure 2.50). Quadrupolar splitting values of 22, 11 and $10 \mathrm{~Hz}$, respectively, are obtained for the final data points recorded after a swelling time of $24 \mathrm{~h}$. Subsequently, significantly higher splitting values are expected in case of the doubled cross-linker concentration, which in general holds true. Nevertheless, the analysis of the polymer with $1 \mathrm{~mol} \%$ cross linker and 2 mol\%o RAFT is hampered by polymer defects.
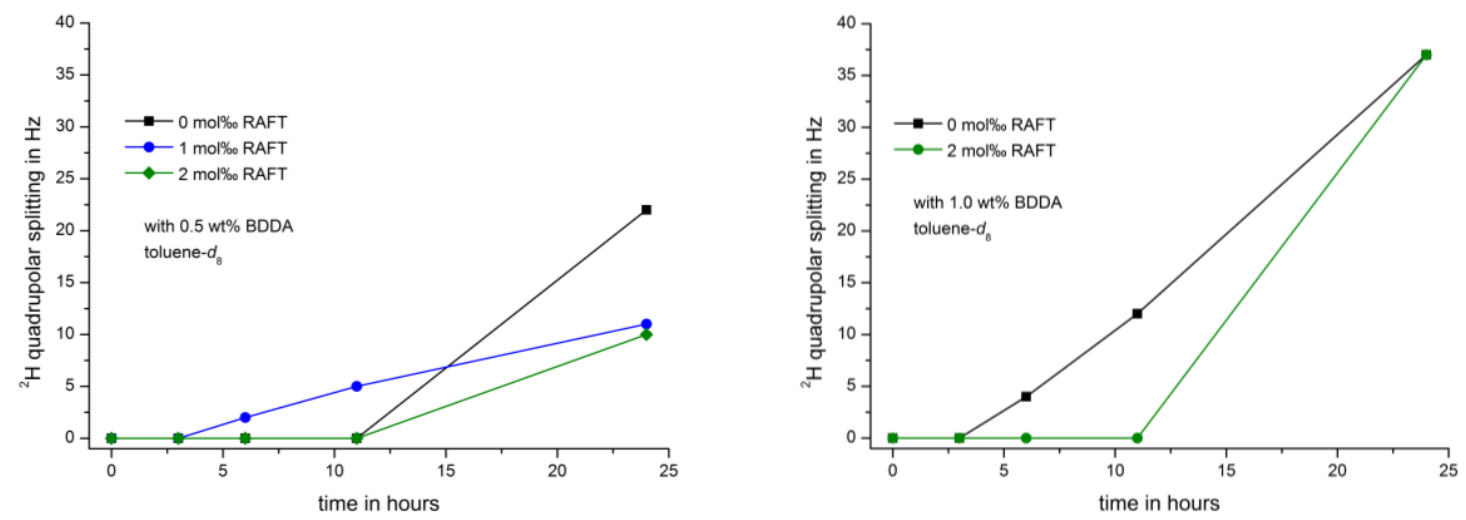

Figure 2.50: Build-up of the methyl ${ }^{2} \mathrm{H}$ quadrupolar splitting of toluene- $d_{8}$ in the middle slice for polymer sticks with 0,1 and 2 mol\%o RAFT obtained in the presence of $0.5 \mathrm{~mol} \%$ BDDA (left) and $1 \mathrm{~mol} \%$ BDDA (right).

In summary, swelling experiments both in acetone- $d_{6}$ and toluene- $d_{8}$ undoubtedly underline that the addition of a chain transfer agent prior to polymerization yields polymeric material with larger or more flexible cavities, which is illustratively proven by smaller quadrupolar splitting values resulting from an altogether smaller degree of alignment. Reduced network formation due to a shifting of the gel effect is thereby nicely represented for small amount of the RAFT agent whereas further increasing the amount of RAFT agent seems to have only a minor effect.

\subsection{Addressing Homogeneity Issues}

Apart from the subjective impression that the desired parameters could be more straightforwardly extracted from spectra of PBA sticks polymerized in the presence of a chain transfer agent, the question of sample homogeneity was also reflected with respect to peak separation, peak form and spatial distribution of the splittings in the resulting ${ }^{2} \mathrm{H}$ slice-selective spectra. 
In the following, the slice-selective ${ }^{2} \mathrm{H}$ NMR spectra of three polymers containing $0.5 \mathrm{~mol} \%$ BDDA with varying amount of RAFT agent and swollen in acetone- $d_{6}$ are depicted in a stacked representation (Figure 2.51).
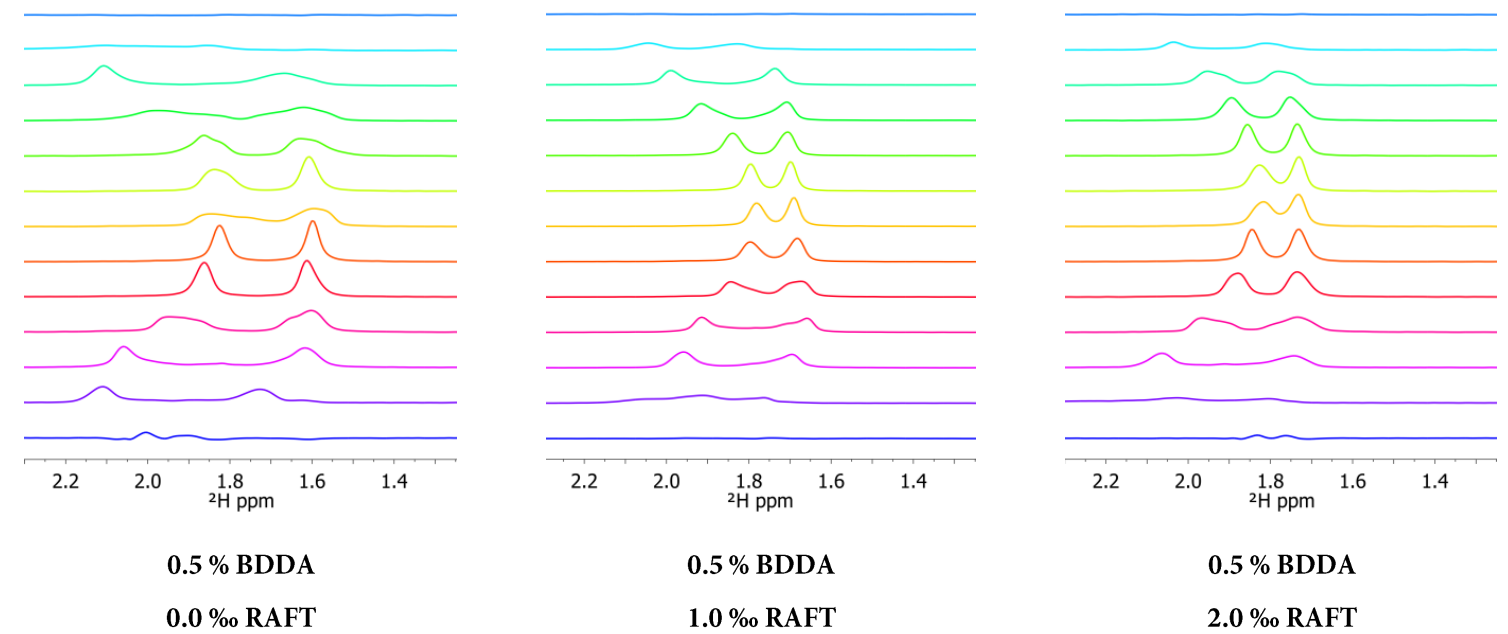

Figure 2.51: Slice-selective ${ }^{2} \mathrm{H}$ NMR spectra of acetone- $d_{6}$ diffusing into PBA sticks ( $\left.0.5 \mathrm{~mol} \% \mathrm{BDDA}\right)$ after $24 \mathrm{~h}$. The sticks were polymerized in the absence of a RAFT agent (left) or in the presence of 1 (middle) or 2 mol\%o RAFT agent (right).

As discussed above, the ${ }^{2} \mathrm{H}$ NMR spectra of the RAFT polymers display smaller quadrupolar splittings, while all slices $(-6 \leq n \leq+6)$ mirror a looser network structure and the resulting smaller degree of alignment. The deviation of the splitting values from uniformity is also smaller for the middle and right stacked plots. Nevertheless, starting from substantially lower values in the first place naturally yields also a narrower distribution of smaller quadrupolar splittings (in absolute number) which in turn would not yet result in a significant improvement of the data precision. However, at higher linker concentration the beneficial effect of the RAFT agent becomes more pronounced. Therefore, two polymer sticks with a higher, but constant amount of cross-linker concentration and varying RAFT concentration ( 0 or 2 mol\%o) are compared in Figure 2.52. Again, polymerization in the presence of a RAFT agent leads to generally

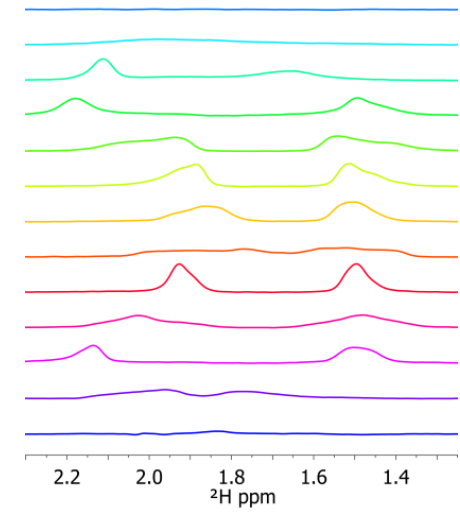

$1.0 \%$ BDDA

$0.0 \%$ RAFT

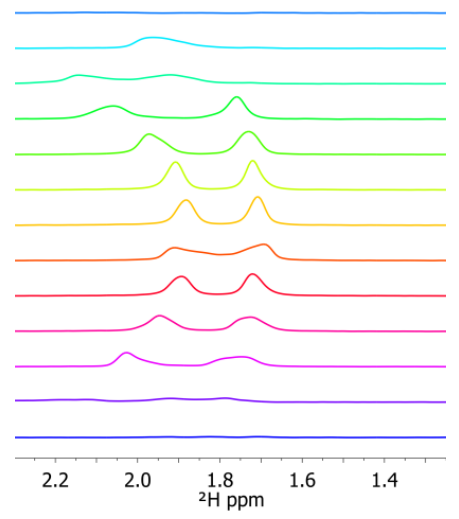

$1.0 \%$ BDDA

$2.0 \%$ RAFT

Figure 2.52: Slice-selective ${ }^{2} \mathrm{H}$ NMR spectra of acetone- $d_{6}$ diffusing into PBA sticks (1 mol\% BDDA) after $24 \mathrm{~h}$. The sticks were polymerized in the absence of a RAFT agent (left) or in the presence of $2 \mathrm{~mol} \%$ chain transfer agent (right). 
smaller quadrupolar splittings yet in this study also to a more defined and uniform signal shape. This might considerably facilitate the interpretation of the spectra and the extraction of anisotropic parameters.

For quantification of the spectral quality the full width at half maximum was extracted for all acetone signals in slice-selective ${ }^{2} \mathrm{H}$ NMR spectra after a swelling time of $24 \mathrm{~h}$. To account for the dependency of the signal broadening on the shim quality and the size of the quadrupolar splitting, the line widths for both acetone- $d_{6}$ signals were added for each slice and divided by the corresponding observed quadrupolar splitting. Moreover, polymer networks with similar quadrupolar splittings were employed for this consideration to yield a dimensionless broadening factor. Quadrupolar splittings of similar size are encountered for polymer sticks with $0.5 \mathrm{~mol} \%$ BDDA without addition of RAFT (12-21 Hz) and with $1.0 \mathrm{~mol} \%$ BDDA polymerized in the presence of $2.0 \mathrm{~mol} \%$ o chain transfer agent $(11-18 \mathrm{~Hz})$. The results of this comparison are depicted in the following (Table 2.3):

Table 2.3: Comparison of the full width at half maximum of two polymer samples ( 0.5 mol\% BDDA with 0 mol\%o RAFT agent (Figure 2.51, left) and 1.0 mol\% BDDA with 2 mol\%o RAFT agent (Figure 2.52 right) displaying similar splitting values. The corresponding spectra were obtained by slice-selective excitation.

\begin{tabular}{c|cccc|} 
& \multicolumn{4}{|c|}{$0.5 \mathrm{~mol} \%$ BDDA, 0.0 mol\%o RAFT } \\
\hline slice & $\begin{array}{c}\Delta v_{1 / 2}(\text { left }) \\
{[\mathrm{Hz}]}\end{array}$ & $\begin{array}{c}\Delta v_{1 / 2}(\text { right }) \\
{[\mathrm{Hz}]}\end{array}$ & $\begin{array}{c}\Delta v_{\mathrm{Q}} \\
{[\mathrm{Hz}]}\end{array}$ & $\frac{\Sigma \Delta v_{1 / 2}}{\Delta v_{\mathrm{Q}}}$ \\
\hline $\mathbf{+ 3}$ & 12.2 & 9.8 & 21 & 1.1 \\
$\mathbf{+ 2}$ & 6.0 & 6.5 & 14 & 0.9 \\
$\mathbf{+ 1}$ & 5.5 & 3.3 & 14 & 0.6 \\
$\mathbf{0}$ & 9.9 & 6.6 & 12 & 1.4 \\
$\mathbf{- 1}$ & 3.0 & 2.6 & 14 & 0.4 \\
$\mathbf{- 2}$ & 3.2 & 3.2 & 16 & 0.4 \\
$\mathbf{- 3}$ & 8.0 & 6.3 & 18 & 0.8
\end{tabular}

\begin{tabular}{|cccc}
\multicolumn{4}{|c}{$1.0 \mathrm{~mol} \%$ BDDA, 2.0 mol\% RAFT } \\
\hline $\begin{array}{c}\Delta v_{1 / 2}(\mathrm{left}) \\
{[\mathrm{Hz}]}\end{array}$ & $\begin{array}{c}\Delta v_{1 / 2}(\mathrm{right}) \\
{[\mathrm{Hz}]}\end{array}$ & $\begin{array}{c}\Delta v_{\mathrm{Q}} \\
{[\mathrm{Hz}]}\end{array}$ & $\frac{\Sigma \Delta v_{1 / 2}}{\Delta v_{\mathrm{Q}}}$ \\
\hline 5.7 & 3.6 & 18 & 0.5 \\
4.2 & 3.8 & 15 & 0.5 \\
3.0 & 2.9 & 11 & 0.5 \\
3.3 & 2.9 & 11 & 0.6 \\
6.9 & 4.5 & 13 & 0.9 \\
3.5 & 3.5 & 11 & 0.6 \\
4.3 & 5.0 & 13 & 0.7
\end{tabular}

The broadening factor varies significantly across the slices for the sample containing only cross-linker $(0.5 \mathrm{~mol} \%)$ but no RAFT agent whilst it is far more evenly spread along the sample with $1 \mathrm{~mol} \% \mathrm{BDDA}$ and $2 \mathrm{~mol} \%$ of the RAFT agent. Furthermore, the values obtained in five of seven slices are noticeably smaller for the material polymerized in the presence of a chain transfer agent (Table 2.3, right). The overall smaller values and the even distribution might be attributed to a network assembled of more uniformly occurring junctions that result in an overall more homogeneous structure of the RAFT-polymerized gel. An analogous trend can be seen for the other samples, yet it is less pronounced and often hampered by the incomplete swelling. 

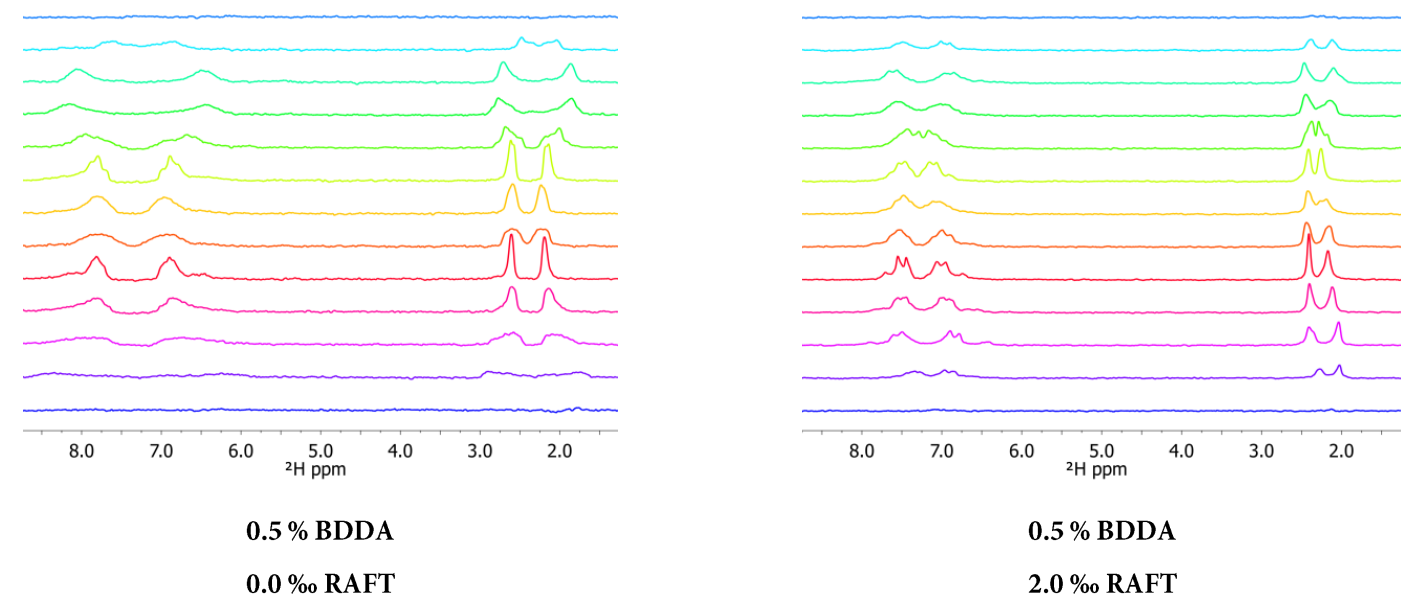

Figure 2.53: Slice-selective ${ }^{2} \mathrm{H}$ NMR spectra of toluene- $d_{8}$ diffusing into PBA (0.5 mol\% BDDA) after 24 h. The sticks were polymerized in the absence (left) or in the presence of 2 mol\%o RAFT (right).

A similar behaviour seems to be indicated by the swelling experiments in toluene- $d_{8}$ albeit with overall more distorted signals. The distortions prevent a straightforward extraction of the full width at half maximum, and longer swelling times would be required to obtain a better spectral quality and thus to discriminate between the individual samples. Nevertheless, a decrease and better uniformity of different quadrupolar splittings is likewise observable for the polymer samples swollen in toluene (Figure 2.53, left to right). Furthermore, increased linker concentrations ( $1 \mathrm{~mol} \%$ instead of $0.5 \mathrm{~mol} \%$ ) seemed to afford less uniform slice-selective spectra, which was also observed for acetone as solvent (compare Figure 2.52 and Figure 2.54).

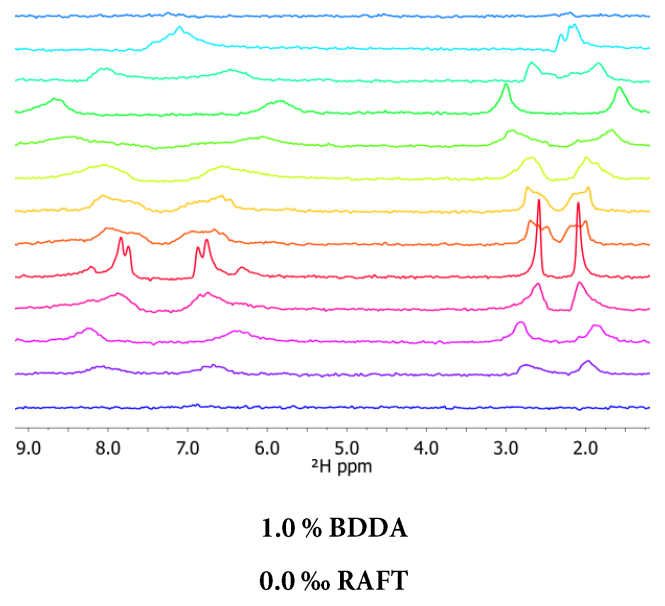

Figure 2.54: Slice-selective ${ }^{2} \mathrm{H}$ NMR spectra of toluene- $d_{8}$ inside of PBA ( $1.0 \mathrm{~mol} \%$ BDDA) sticks recorded $24 \mathrm{~h}$ after sample preparation.

In summary, slice-selective NMR spectroscopy is a very versatile tool to gain insight into the network structure of polymeric material. The measurement does not require any special equipment and allows drawing conclusions about the relative pore size as well as the local polymer structure and its homogeneity across the sample. The presence of a chain transfer agent retards cross-linking thus resulting in larger cavities and decreased alignment strength. On the one hand, this may be an interesting tuning possibility and it also seems to provide a significantly more homogeneous network composition on the other hand. Thereby, a significantly better peak separation and spectrum quality can be obtained for the measurement of complex molecules aligned in a polymer environment. 
Nevertheless, these results have to be confirmed in further measurements with variable inner diameter of the polymer sticks to avoid having to cut out a part and thereby destroying the surface. The softer PBA material is also more labile than the rigid polystyrene sticks because the faster swelling of PBA leads to considerably more strain that is induced on the polymer at a given time interval. Regarding the swelling times, longer monitoring of the swelling seems beneficial, because although the concentration and splitting profiles illustrate that considerable swelling has taken place $24 \mathrm{~h}$ after sample preparation, they also indicate that the solvent uptake is still not complete and the gel not fully equilibrated. Longer swelling times (e.g. $48 \mathrm{~h}$ ) might be advantageous for an exact correlation of the data from different polymers and even more substantial for an adequate evaluation of the data obtained by swelling in toluene whose interpretation is hampered by an overall poorer signal quality.

\subsubsection{Synthesis and Application of RAFT-polymerized PS Gels}

In consideration of the very promising results presented for butyl acrylate polymers prepared by RAFT polymerization the transferability of these findings to the polystyrene networks has to be investigated. The usefulness of PS sticks for the analysis of reactive compounds was underlined in chapter 2.2.1 and homogeneous PS-RAFT might turn out very beneficial assuming that the resulting RAFT network will still withstands the harsh conditions upon addition of, e.g. organolithiums.

According to the usual procedure of thermal polymerization polystyrene sticks with varying cross-linker concentrations of $0.2,0.3$ and 0.4 vol\% DVB with 0.35 mol\%o RAFT agent (CDB: cyanopropyldithiobenzoate) were prepared, while for a cross-linker concentration of $0.3 \mathrm{vol} \%$ DVB also two samples with 0.7 mol\%o RAFT agent were employed. The samples are nicely distinguishable by the increasing colouring of the obtained material (Figure 2.55).
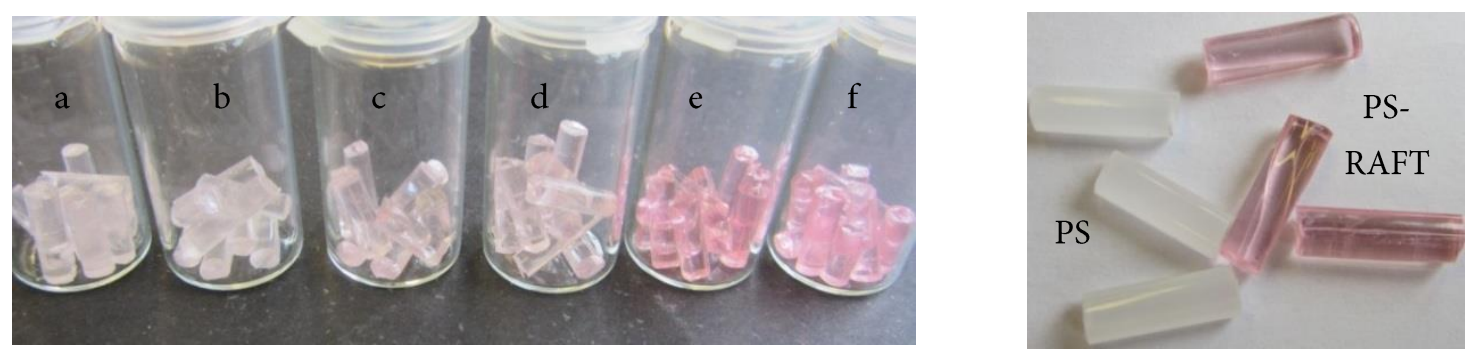

Figure 2.55: Photographs of the different PS / RAFT sticks (left) with different amounts of RAFT agent (a-d $0.35 \mathrm{~mol} \%$, e and f $0.7 \mathrm{~mol} \%$ ) and comparison to the pure PS sticks (right).

Due to the presence of the chain transfer agent, all PS / RAFT polymer sticks appear slightly rose-coloured with the intensity being proportional to the employed amount of 
CDB (Figure 2.55, left). Samples a-d varied in the amount of cross-linker but all contain $0.35 \mathrm{~mol} \%$ o RAFT agent while the more intensely coloured PS sticks e and $\mathrm{f}$ contain the double amount of RAFT agent $(0.7 \mathrm{~mol} \%)$. The quality of the sticks was very good and they were also cut into pieces of approximately $1 \mathrm{~cm}$ length. Compared to the pure polystyrene material depicted on the right, the PS / RAFT sticks are coloured yet less turbid which may be attributed to a smaller amount of spatial heterogeneity (less light scattering). However, more transparent sticks were also obtained in free radical styrene polymerization and attributed to polymeric material that contained a larger amount of unreacted monomer, which was likewise verified to be present for the herein analysed PS / RAFT sticks (Figure 2.56).

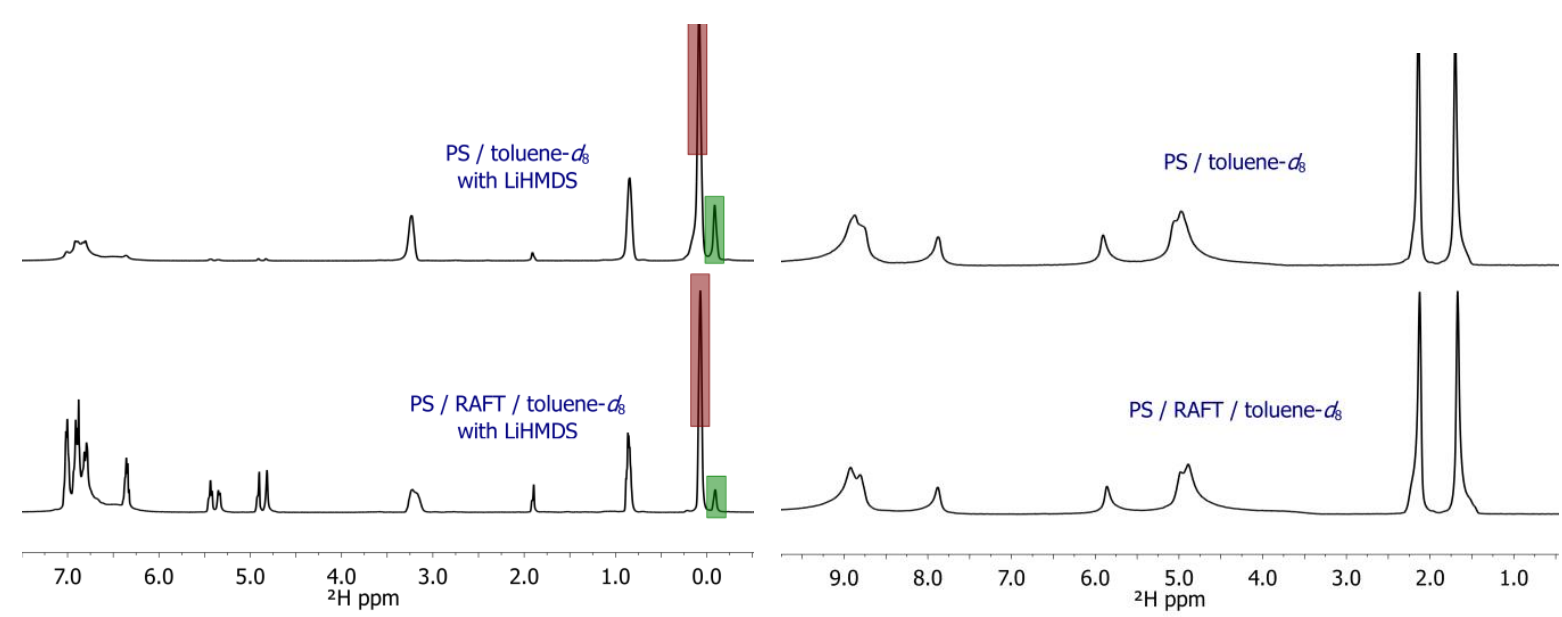

Figure 2.56: ${ }^{1} \mathrm{H}-\mathrm{CPMG}$ spectra of LiHMDS (4) in PS/ toluene- $d_{8}$ (top) and PS/ RAFT/ toluene- $d_{8}(0.3$ vol\% DVB, 0.7 mol\% RAFT) (bottom) depicted on the left and corresponding ${ }^{2} \mathrm{H}$ quadrupolar splittings on the right. Red boxes mark the LiHMDS signal while the decomposition product is highlighted by green boxes.

On the left of Figure 2.56 the ${ }^{1} \mathrm{H}-\mathrm{CPMG}$ spectra of LiHMDS (4) in toluene- $d_{8}$ diffused into pure PS (top) or PS / RAFT (bottom) clearly illustrate that the pure PS material contains less unreacted monomer (signals between 5-6.5 ppm). Both spectra further display signals representing $\mathrm{Et}_{2} \mathrm{O}$ (due to synthesis of 4 in $\mathrm{Et}_{2} \mathrm{O}$ ) and LiHMDS (red signal). The small signal next to the LiHMDS (green) is the corresponding decomposition product. The ratio of LiHMDS to decomposition product is definitely smaller (9:1) in case of PS / RAFT sticks compared to pure PS (5.3:1). Although deviations may be present due to sample preparation, this clearly shows that the polymeric material containing RAFT (dithio-) moieties is still stable towards organometallic compounds. Another indicator for its stability is the corresponding ${ }^{2} \mathrm{H}$ quadrupolar splitting presented on the right of Figure 2.56. Both samples show a similar signal form and splitting values which further underlines that the alignment is preserved and the polymer environment not attacked by the LiHMDS molecules. 
The PS / RAFT gels were also analysed by slice-selective ${ }^{2} \mathrm{H}$ NMR experiments five days after samples preparation and yielded good concentration and quadrupolar splitting profiles. To compare these spectra with the pure PS sticks, a sample with similar ${ }^{2} \mathrm{H}$ quadrupolar splitting (c.f. 2.2.2) was chosen analogously to the proceeding described for PBA and PBA / RAFT polymers. The full width at half maximum of the components of the quadrupolar doublet of the methyl group was measured and the obtained values are depicted in the following table.

Table 2.4: Comparison of the full width at half maximum of two polystyrene samples (both containing $0.3 \mathrm{vol} \% \mathrm{DVB}$ ) with $0.7 \mathrm{~mol} \%$ RAFT (left) after five days of swelling and $0.0 \mathrm{~mol} \%$ RAFT (right) seven days after sample preparation. The corresponding spectra were obtained by slice-selective excitation.

\begin{tabular}{c|cccc|} 
& \multicolumn{4}{|c|}{ PS: 0.3 vol\% DVB, 0 mol\%o RAFT } \\
\hline slice & $\begin{array}{c}\Delta v_{1 / 2}(\text { left }) \\
{[\mathrm{Hz}]}\end{array}$ & $\begin{array}{c}\Delta v_{1 / 2}(\text { right }) \\
{[\mathrm{Hz}]}\end{array}$ & $\begin{array}{c}\Delta v_{\mathrm{Q}} \\
{[\mathrm{Hz}]}\end{array}$ & $\frac{\Sigma \Delta v_{1 / 2}}{\Delta v_{\mathrm{Q}}}$ \\
\hline $\mathbf{+ 3}$ & 9.2 & 8.2 & 24.0 & 0.7 \\
$\mathbf{+ 2}$ & 5.9 & 5.0 & 23.0 & 0.5 \\
$\mathbf{+ 1}$ & 3.9 & 3.6 & 22.0 & 0.3 \\
$\mathbf{0}$ & 2.9 & 3.6 & 21.0 & 0.3 \\
$\mathbf{- 1}$ & 2.4 & 3.7 & 21.0 & 0.3 \\
$\mathbf{- 2}$ & 3.4 & 5.4 & 22.0 & 0.4 \\
$\mathbf{- 3}$ & 7.0 & 8.5 & 24.0 & 0.6
\end{tabular}

\begin{tabular}{|cccc}
\multicolumn{4}{|c}{ PS: 0.3 vol\% DVB, 0.7 mol\%o RAFT } \\
\hline $\begin{array}{c}\Delta v_{1 / 2}(\text { left }) \\
{[\mathrm{Hz}]}\end{array}$ & $\begin{array}{c}\Delta v_{1 / 2}(\text { right }) \\
{[\mathrm{Hz}]}\end{array}$ & $\begin{array}{c}\Delta v_{\mathrm{Q}} \\
{[\mathrm{Hz}]}\end{array}$ & $\frac{\Sigma \Delta v_{1 / 2}}{\Delta v_{\mathrm{Q}}}$ \\
\hline 8.3 & 7.6 & 22.0 & 0.7 \\
6.0 & 4.8 & 22.0 & 0.5 \\
3.4 & 4.1 & 21.0 & 0.4 \\
3.3 & 5.3 & 20.0 & 0.4 \\
5.1 & 6.1 & 22.0 & 0.5 \\
3.1 & 5.9 & 23.0 & 0.4 \\
3.2 & 6.5 & 23.0 & 0.4
\end{tabular}

Both gels contain the same amount of cross-linker ( $0.3 \mathrm{vol} \%)$, but the gel characterized on the left of Table 2.4 is polymerized in the presence of $0.7 \mathrm{~mol} \%$ RAFT agent. This gel was analysed after five days of swelling, whereas entries on the left table correspond to a pure PS gel seven days after sample preparation. As a result, a comparable size of the ${ }^{2} \mathrm{H}$ quadrupolar splittings and a similar data quality can be achieved at shorter swelling times. Thus, PS / RAFT gels seem to be at least equally appropriate for utilization as alignment medium of organometallic compounds. To further assure this, new sticks with defined cross-linker and higher RAFT concentrations have to be synthesized and subsequently employed to a swelling study as already described for the different pure PS materials. Furthermore, low-temperature experiments have to be carried out to estimate the temperature limits of these polymer gels. Measurement of more complex compounds inside these PS / RAFT gels could be beneficial if, at identical swelling times, better peak separation is obtained.

Considering all those aspects, slice-selective NMR experiments proved to be very useful for the analysis of polymer networks, and the employment of a RAFT agent to modify the 
existing PS and PBA gels seems to be a promising approach to improve the quality of the gels for SAG applications.

\subsubsection{Interim Conclusion}

In this chapter the application of strain-induced alignment in a gel (SAG) method to organolithium compounds was investigated and discussed along with further investigations on the swelling behaviour of polystyrene (PS), polybutyl acrylate (PBA) and RAFT-polymerized polybutyl acrylate sticks.

It could be illustrated that PS polymerized in the absence of a radical starter is able to withstand the harsh conditions brought about by the addition of highly reactive organometallic compounds. Diffusion of several organolithium compounds into the gel could be observed, and the ${ }^{7} \mathrm{Li}$ residual quadrupolar couplings (RQCs) proved to be a very valuable tool for the discrimination between aggregates of high (hexameric, tetrameric) and low (dimeric, monomeric) symmetry due to the appearance of quadrupolar triplets for the latter while the singlet in the ${ }^{7} \mathrm{Li}$ NMR spectrum is preserved for the former.

The method is not only applicable to ${ }^{7} \mathrm{Li}$ but also to other quadrupolar nuclei, e.g. ${ }^{2} \mathrm{H},{ }^{11} \mathrm{~B}$, ${ }^{25} \mathrm{Na},{ }^{27} \mathrm{Al}$. The ${ }^{7} \mathrm{Li}$ and ${ }^{11} \mathrm{~B} \mathrm{NMR}$ spectra of $\mathrm{LiBF}_{4}(6)$ in PS / THF- $d_{8}$ are displayed in Figure 2.57. In each spectrum a clean quadrupolar triplet is observed indicating that both lithium and boron are part of aggregates with low symmetry in THF-solution. The possibility to judge on the aggregation of boron compounds in solution could also be very beneficial for the investigation of structural features of frustrated Lewis
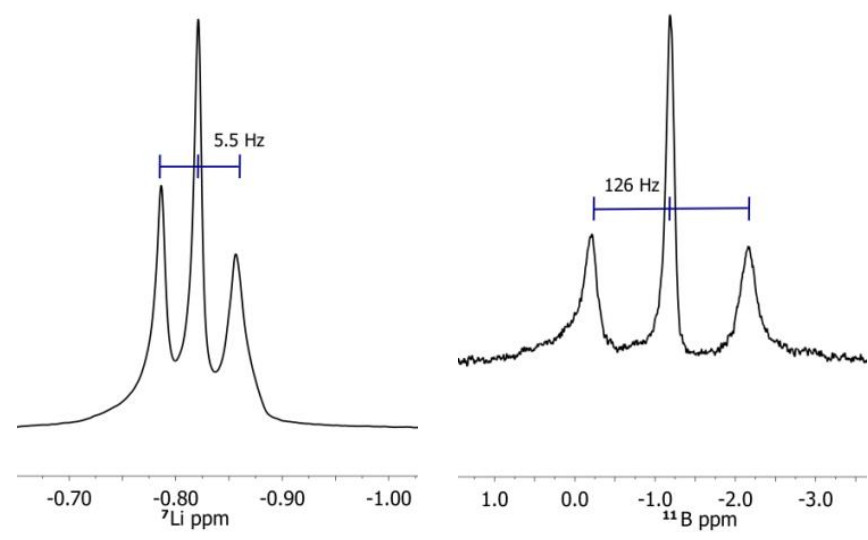

Figure 2.57: ${ }^{7} \mathrm{Li}$ and ${ }^{11} \mathrm{~B}$ NMR spectrum of $\mathrm{LiBF}_{4}(6)$ in PS / THF- $d_{8}(0.3$ vol\% DVB).

pairs. ${ }^{[192]}$

Slice-selective excitation is a very versatile method to gain information on the diffusion of solvent molecules and analytes into the polystyrene network as well as the homogeneity of the gel with spatial resolution. It was used to characterize another polymer gel, polybutyl acrylate (PBA) that is compatible with a larger number of solvents but not suited for the application to reactive organometallic compounds. Certainly, PBA displays better lowtemperature properties allowing characterization of otherwise flexible molecules or 
equilibria. In a comparative study, the homogeneity of different PBA gels polymerized in the absence or presence of a RAFT agent was addressed by slice-selective deuterium NMR experiments, which might be also a valuable instrument for the investigation of polymer networks in general. First PS / RAFT gels were also analysed.

Apart from deeper insights into the methodology itself, the connection of the two approaches, isotropic and anisotropic NMR spectroscopic studies, should be pursued in order to address more complex compounds and determine their structure in solution. The following chapter will be concerned with combined studies for the structure elucidation of Grignard and Turbo-Grignard compounds as well as the lithium lithiate $\left.[\text { Li (diglyme })_{2}\right]\left[(\right.$ diglyme $\left.) \mathrm{Li}_{2}\left(\mathrm{C}_{4} \mathrm{H}_{3} \mathrm{~S}\right)_{3}\right]$ in solution. 


\subsection{Combined Studies for Structure Elucidation}

\subsubsection{Study of $i \mathrm{PrMgCl}$ and its Turbo Analogue in THF}

\subsubsection{Structural Motifs of Grignard and Turbo-Grignard Compounds}

Grignard reagents are alkyl- or aryl-magnesium halides first introduced by Victor Grignard at the beginning of the $20^{\text {th }}$ century. ${ }^{[193]}$ They range among the most frequently used organometallic reagents and are often employed for $\mathrm{C}-\mathrm{C}$ bond formation (e.g. through nucleophilic substitution or addition to a carbonyl moiety). Although with slightly lower reactivity than common organolithium compounds, they are still strong nucleophiles which display only moderate functional group tolerance at low temperatures. Owing to the wide application of Grignard reagents in synthetic chemistry, structure elucidation of the involved species has been playing an important role ever since their discovery. ${ }^{[194]}$ The very complex composition in solution is summarized by the Schlenk equilibrium depicted in Scheme 2.8. ${ }^{[195]}$

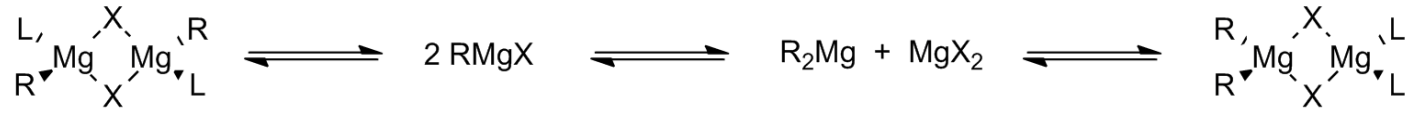

Scheme 2.8: Schlenk equilibrium summarizing the complex behaviour of Grignard reagents in solution (with $\mathrm{R}$ being an organic residue, $\mathrm{X}$ a halide atom and $\mathrm{L}$ a Lewis base).

The equilibration between the alkyl or aryl-magnesium halide $\mathrm{RMgX}$, the corresponding magnesium halide salt $\mathrm{MgX}_{2}$ and the dialkyl- or diaryl-magnesium compound $\mathrm{R}_{2} \mathrm{Mg}$ is extremely fast at room temperature and therefore no distinct signals for the different species can be obtained by NMR spectroscopy. Furthermore, the equilibrium depends on the solvent, concentration and temperature along with the nature of the organic residue and the employed halide. Therefore, an investigation of Grignard reagents in solution is anything but straightforward and results obtained for a certain composition cannot be easily generalized. Early studies concerning the equilibrium and the determination of the equilibrium constant $K$ were carried out on the basis of $\operatorname{IR}^{[196]}$ and $\mathrm{NMR}^{[197]}$ measurements. An almost statistical distribution of the species involved in the Schlenk equilibrium was therein concluded for Grignard reagents with $\mathrm{R}=\mathrm{Me}, \mathrm{Et}, t \mathrm{Bu}$ and $\mathrm{Ph}$. An additional binuclear, $\mu-\mathrm{Cl}_{3}$-bridged structure (Scheme 2.9) was promoted by Sakamoto and coworkers on the basis of crystal structures and ESI measurements of different Grignard compounds. ${ }^{[198]}$ However, a drawback of these measurements is that no intact Grignard compounds, but only species which suffer from hydride abstraction are detected. Furthermore, the crystal structure contains an excess of the halide. 
2

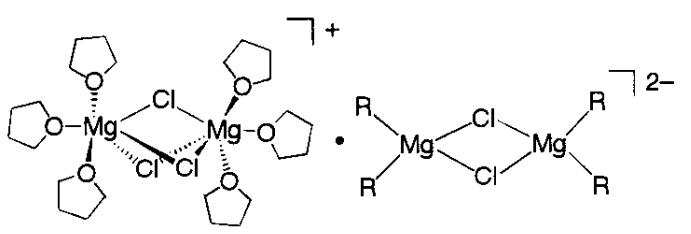

Scheme 2.9: Crystal structure of $2\left[\mathrm{Mg}_{2}(\mu-\mathrm{Cl})_{3} \mathrm{THF}_{6}\right]^{+} \cdot\left[\mathrm{R}_{4} \mathrm{Mg}_{2} \mathrm{Cl}_{2}\right]^{2-}(\mathrm{R}=\mathrm{Ph}) \cdot{ }^{[198]}$

The research on Grignard reagents was taken to the next stage by the work of Knochel et al. who investigated the effect of metal halides on the reactivity of Grignard reagents. ${ }^{[199]}$ Upon addition of metal halides, for instance $\mathrm{LiCl}$, prior to the Grignard reaction they could demonstrate an impressive improvement in terms of reaction time and yield accompanied by an increased functional group tolerance. This behaviour accounts for their name "turbo Grignard", whose best studied representatives comprise isopropyl residues. The group of Knochel performed calculations aiming at the identification of transition states and intermediates of halogen-metal exchange reactions and ascribed the enhanced reaction progress to different "ate"-type species linked through the following equilibrium (Scheme 2.10). ${ }^{[200]}$

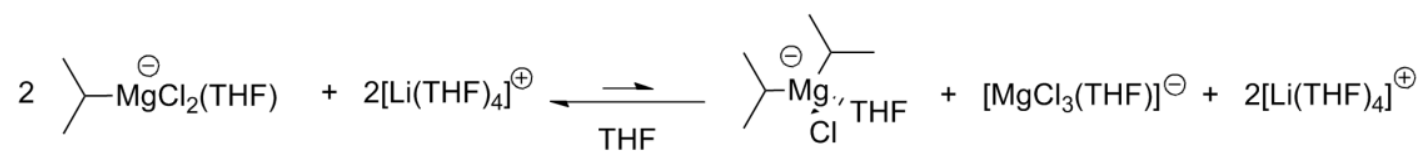

Scheme 2.10: Anionic Schlenk equilibrium also referred to as "magnesiate equilibrium" proposed by Knochel and co-workers. ${ }^{[200]}$

Crystallographic studies by Lerner et al. aimed at characterizing $i \mathrm{PrMgCl} \cdot \mathrm{LiCl}$ to find a structural proof for the observed LiCl-dependent "magnesiate effect". [201] While crystallographic proof for the coordination mode $\left[\mathrm{RMg}(\mu-\mathrm{Cl})_{2} \mathrm{Li}\right]$ is available for the related "turbo Hauser" bases (TMP)MgCl.LiCl, ${ }^{[202]}$ Lerner and co-workers did not obtain any crystal structure of isopropyl magnesium chloride in which lithium chloride is incorporated. Instead, the open-cube $\mathrm{MgCl}_{2}$ adduct $[i \operatorname{PrMgCl}(\mathrm{THF})]_{2}\left[\mathrm{MgCl}_{2}(\mathrm{THF})_{2}\right]_{2}$ was observed (Figure 2.58). This species is isostructural to crystalline compounds obtained from pure $\mathrm{RMgCl}$ solutions $(\mathrm{R}=\mathrm{Me}, t \mathrm{Bu}, \mathrm{Ph}, \mathrm{Bn}){ }^{[198,203]}$

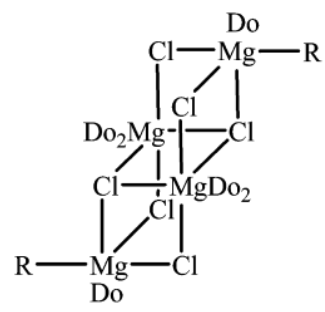

Figure 2.58: Open-cube $\mathrm{MgCl}_{2}$-adduct crystallized from a THF solution of $i \mathrm{PrMgCl} \cdot \mathrm{LiCl}$ layered with $\mathrm{Et}_{2} \mathrm{O} \cdot[201]$ 
As Grignard reagents play such a vital role in organic and inorganic synthesis it is of great interest to know more about the structural properties of the reactive species. In the following, results from a combined NMR and mass spectrometric analysis of $i \operatorname{PrMgCl}(7)$ and $i \mathrm{PrMgCl} \cdot \mathrm{LiCl}(8)$ in THF are presented that were obtained in collaboration with Christoph Schnegelsberg from the workgroup of Prof. K. Koszinowski who carried out the ESI mass spectrometry measurements.

\subsubsection{Electrospray Ionisation (ESI) Mass Spectrometry}

ESI mass spectrometry measurements of $i \operatorname{PrMgCl}(7)$ in THF yielded mostly trinuclear anionic species, namely $\mathrm{Mg}_{3} \mathrm{Cl}_{3}\left(\mathrm{C}_{3} \mathrm{H}_{7}\right)_{4}^{-}(\mathrm{m} / z 351$ for the main isotopologue) and $\mathrm{Mg}_{3} \mathrm{Cl}_{2}\left(\mathrm{C}_{3} \mathrm{H}_{7}\right)_{5}{ }^{-}(\mathrm{m} / z 359$ for the main isotopologue). The observed main species all contain three magnesium atoms accompanied by chlorine atoms and organic residues. Neither the presence of THF nor of any lithium atoms is observed therein, but both represent integral parts of the cationic species. Addition of $\mathrm{LiCl}$ to form the turbo compound $i \mathrm{PrMgCl} \cdot \mathrm{LiCl}$ (8) did result in an increased number of counts attributed to a higher ionic character which was further underlined by conductivity measurements. The overall composition is however preserved and analogous mass peaks for the anionic species were observed for 8 in THF.

\subsubsection{Isotropic NMR Experiments}

Solutions of $0.3 \mathrm{~mL} i \operatorname{PrMgCl}(7,1.35 \mathrm{M})$ and $i \operatorname{PrMgCl} \cdot \mathrm{LiCl}(\mathbf{8}, 0.95 \mathrm{M})$ in THF were diluted by addition of $0.4 \mathrm{~mL}$ THF- $d_{8}$ yielding concentrated samples for first isotropic NMR spectroscopic measurements. The resulting ${ }^{1} \mathrm{H}$ NMR spectra are depicted in Figure 2.59.

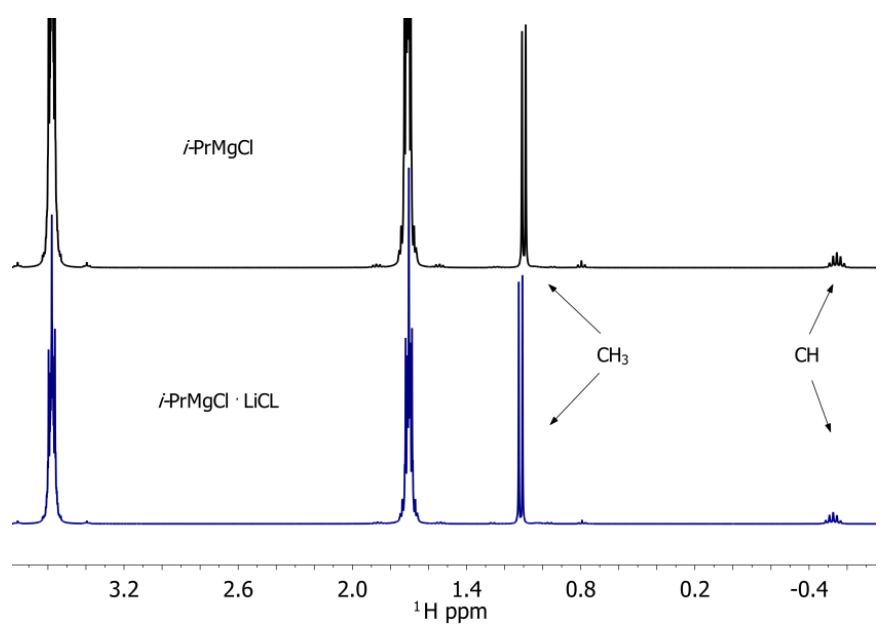

Figure 2.59: ${ }^{1} \mathrm{H}$ NMR spectra of $i \mathrm{PrMgCl}(7)$ and $i \mathrm{PrMgCl} \cdot \mathrm{LiCl}(8)$ in THF- $d_{8}$. Signals corresponding to the isopropyl groups are highlighted. 
The ${ }^{1} \mathrm{H}$ NMR spectra of $\mathbf{7}$ and $\mathbf{8}$ are very similar and show only one signal set at room temperature. The doublets assigned to the $\mathrm{CH}_{3}$ groups show a coupling constant of ${ }^{3} J_{\mathrm{HH}}=7.8 \mathrm{~Hz}(7)$ and $7.7 \mathrm{~Hz}(8)$ and resonate at 1.12 and $1.09 \mathrm{ppm}$, respectively. The signal for the $\mathrm{CH}$-group attached to the magnesium atom is observed at negative values $(-0.53$ and $-0.55 \mathrm{ppm}$ ). These small chemical shift differences for the $\mathrm{CH}$-group are also present in the corresponding ${ }^{13} \mathrm{C}$ NMR spectra. The ${ }^{7} \mathrm{Li} \mathrm{NMR}$ spectrum of the turbo Grignard 8 shows one resonance at $0.02 \mathrm{ppm}$. No signals were obtained for the ${ }^{25} \mathrm{Mg}$ nucleus in the employed concentration region.

Subsequently, low temperature NMR experiments of $\mathbf{7}$ and $\mathbf{8}$ were conducted by cooling both solutions in steps of $25^{\circ} \mathrm{C}$. Starting from $25^{\circ} \mathrm{C}$, measurements were carried out at $0{ }^{\circ} \mathrm{C},-25^{\circ} \mathrm{C}$ and $-50{ }^{\circ} \mathrm{C}$. Below $-50{ }^{\circ} \mathrm{C}$ the samples were frozen and no spectra could be acquired. The ${ }^{1} \mathrm{H}$ NMR spectra at $-50{ }^{\circ} \mathrm{C}$ are illustrated in Figure 2.60. For both compounds, a second signal set has appeared (green bars) very close to and with identical splitting compared to the parent signal (red bars). Interestingly, the intensity of the second signal set is considerably higher in case of the turbo reagent $i \mathrm{PrMgCl} \cdot \mathrm{LiCl}(\mathbf{8})$. Integration yields an integral ratio (red:green) of $1: 0.2$ in case of $i \operatorname{PrMgCl}(7)$ and of $1: 0.5$ for iPrMgCl.LiCl (8). The new signals may be due to a dialkyl magnesium species whose existence is promoted by the addition of $\mathrm{LiCl}$. However, the determination of the exact nature of the participating species by diffusion or NOE measurements was hampered by the fast exchange still present at $-50^{\circ} \mathrm{C}$.

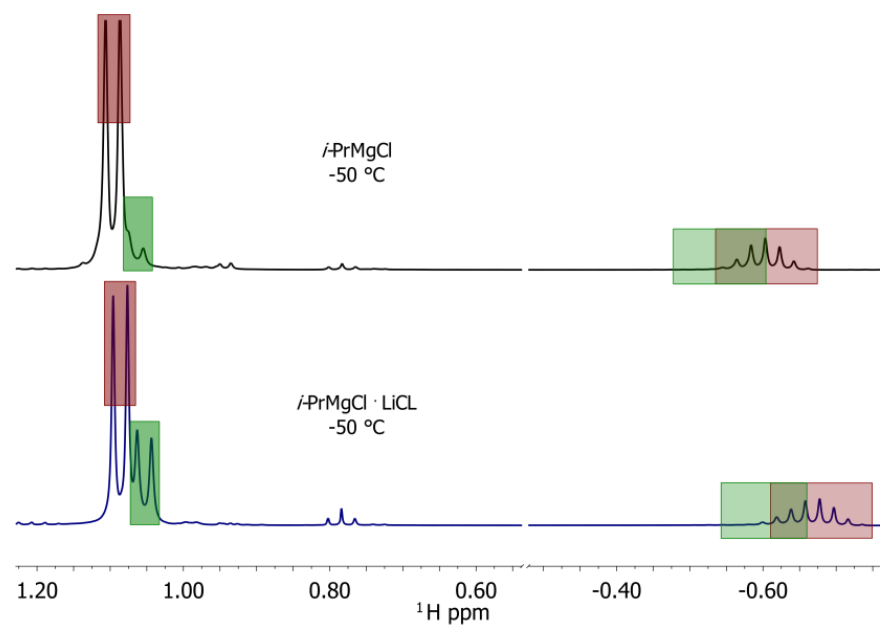

Figure 2.60: ${ }^{1} \mathrm{H}$ NMR spectra of $i \mathrm{PrMgCl}(7)$ and $i \mathrm{PrMgCl} \cdot \mathrm{LiCl}(8)$ in THF- $d_{8}$ recorded at $-50{ }^{\circ} \mathrm{C}$. Red boxes represent the parent signal set, green boxes the second signal set arising at lower temperatures.

Accordingly, a ${ }^{7} \mathrm{Li}$ NMR spectrum of 8 was recorded at $-50{ }^{\circ} \mathrm{C}$, which still displayed only one sharp signal. The observation of two separate signals could be impeded by either the 
${ }^{7} \mathrm{Li}$ atoms being part of an equilibrium which is still fast at the measurement temperature or by the ${ }^{7} \mathrm{Li}$ atoms not being involved in the formation of the second species.

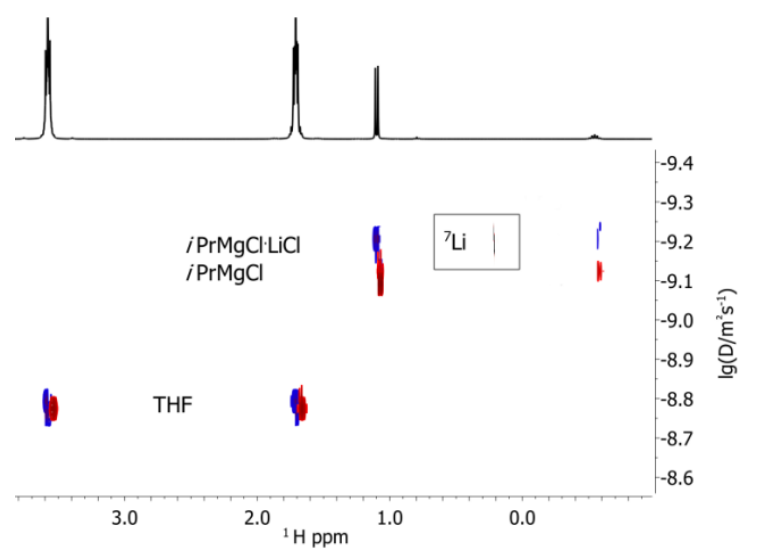

Figure 2.61: Overlay of the ${ }^{1} \mathrm{H}-\mathrm{DOSY}$ spectra of $\mathbf{7}$ (red) and $\mathbf{8}$ (blue) with the ${ }^{7} \mathrm{Li}-\mathrm{DOSY}$ spectrum of 8 .

To investigate whether $\mathrm{LiCl}$ promotes the formation of higher aggregates, ${ }^{1} \mathrm{H}$ and ${ }^{7} \mathrm{Li}$ DOSY was employed at room temperature. The ${ }^{1} \mathrm{H}$-DOSY spectra of $\mathbf{7}$ (red) and 8 (blue) (Figure 2.61) exhibit a slightly different diffusion behaviour for both samples. According to the overlay of the two spectra, the size of the turbo reagent is somewhat larger than that of the standard isopropyl magnesium chloride. Thus, the addition of lithiumchloride does have a pronounced effect on the average molecular size either by coordinating to the $i \operatorname{PrMgCl}-$ fragment or by interfering with a possible equilibrium. The former might be supported by the ${ }^{7} \mathrm{Li}$ diffusion coefficient which is identical to the corresponding ${ }^{1} \mathrm{H}$ diffusion value. In order to analyse the concentration dependence of $\mathrm{D}$, samples with significantly reduced quantities of 7 and 8 ( $3 \mathrm{mg}$ per $0.5 \mathrm{~mL}$ ) were prepared along with a reference sample of $\mathrm{LiCl}$ in THF- $d_{8}(9)$, which is known to predominantly display a dimeric structure. ${ }^{[1,204]}$ Anthracene $(\mathrm{M}=178 \mathrm{~g} / \mathrm{mol})$ and benzene- $d_{6}(\mathrm{M}=84 \mathrm{~g} / \mathrm{mol})$ were employed as reference compounds to allow an estimation of the average molecular weights of $\mathbf{7}$ and $\mathbf{8}$. An overlay of the resulting ${ }^{1} \mathrm{H}$ - and ${ }^{7} \mathrm{Li}-\mathrm{DOSY}$ spectra is depicted in Figure 2.62. 


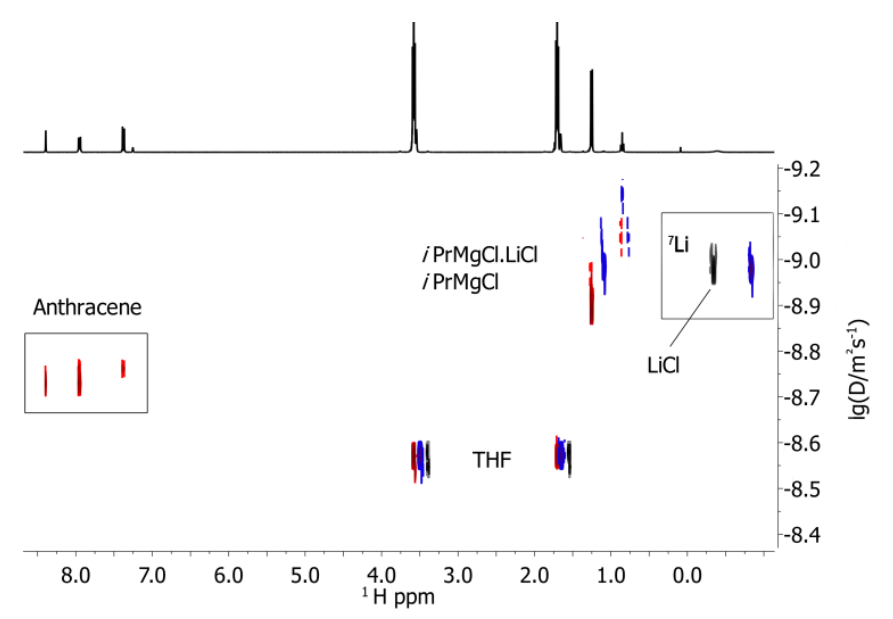

Figure 2.62: Overlay of the ${ }^{1}$ H-DOSY spectra of 7 (red), 8 (blue) and 9 (black) in THF- $d_{8}$ with the corresponding ${ }^{7} \mathrm{Li}$-DOSY spectra of 8 and $\mathbf{9}$. All spectra were measured at room temperature with $3 \mathrm{mg}$ of the particular compound.

Due to the lower solute concentration and thus reduced solution viscosity, all diffusion coefficients display higher values compared to the spectra depicted in Figure 2.61. A diffusion coefficient of $D=1.36 \cdot 10^{-9} \mathrm{~m}^{2} / \mathrm{s}$ could be extracted for the pure isopropyl Grignard compound (red) while the diffusion coefficient for the Turbo compound (blue) was again higher $\left(D=1.12 \cdot 10^{-9} \mathrm{~m}^{2} / \mathrm{s}\right)$. The molecular size difference of 7 and 8 is independant of the concentration, which could be verified by an overlay of the corresponding DOSY spectra at high and low concentration. The comparison revealed that dilution only scales all values whereas the relative position of 7 towards 8 remains unaffected. In agreement with the concentrated sample, the value found for the ${ }^{7} \mathrm{Li}$ diffusion coefficient of $\mathbf{8}$ is again identical to the extracted ${ }^{1} \mathrm{H}$ diffusion coefficient. Interestingly, identical ${ }^{7} \mathrm{Li}$ diffusion coefficients were again observed for 8 and $\mathbf{9}$ in THF- $d_{8}$. This means, that either

(1) $\mathrm{LiCl}$ is not directly incorporated into the structure of $\mathbf{8}$ and thus displays the same diffusion value as $\mathbf{9}$ while the lithium-free magnesium compounds exist in a slightly different equilibrium or

(2) a joined aggregate consisting of both magnesium and lithium is present in the solution of $\mathbf{8}$, which accidentally displays the same diffusion coefficient as the THFcoordinated $\mathrm{LiCl}$ dimer

For the present composition the coincidence of the diffusion coefficients of particularly distinct sample mixtures impedes an exact evaluation of the diffusion experiments. Employment of a lithium salt with different molecular weight might solve this inconvenience. Another drawback is the equilibrium supposedly present for both $\mathbf{7}$ and $\mathbf{8}$ 
in THF. This would mean that at room temperature only the averaged diffusion coefficient for all rapidly interchanging aggregates is observed. With the help of anthracene, benzene$d_{6}$ and THF- $d_{8}$ as references, the molecular weight corresponding to the two diffusion coefficients extracted from the spectra shown in Figure 2.62 could be estimated. Thereby, an average molecular weight of $\sim 260 \mathrm{~g} / \mathrm{mol}$ was calculated for the $i \mathrm{PrMgCl}$ sample whereas $\sim 300 \mathrm{~g} / \mathrm{mol}$ was determined as molecular weight for the $i \mathrm{PrMgCl} \cdot \mathrm{LiCl}-$ species.

A summary of several conceivable complexes discussed in the beginning of this chapter is depicted in Scheme 2.11.

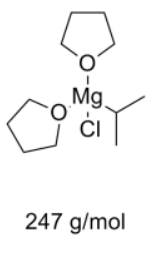

(i)

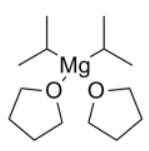

$255 \mathrm{~g} / \mathrm{mol}$

(ii)

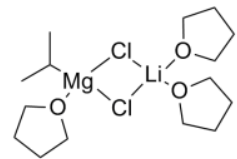

$362 \mathrm{~g} / \mathrm{mol}$

(iii)

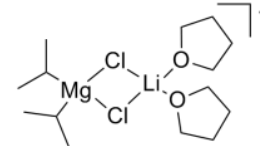

$333 \mathrm{~g} / \mathrm{mol}$

(iv)

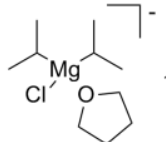

$218 \mathrm{~g} / \mathrm{mol}$

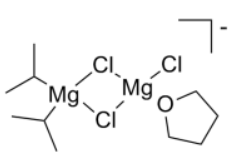

$313 \mathrm{~g} / \mathrm{mol}$

(vi)

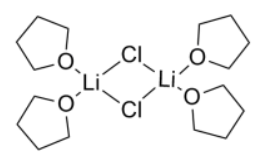

$373 \mathrm{~g} / \mathrm{mol}$ $(\mathbf{v})$

Scheme 2.11: Conceivable structural motifs discussed in literature for Grignard and Turbo-Grignard compounds in solution.

The monomeric di-solvated structure (i) as well as the dialkyl magnesium compound (ii), both integral parts of the Schlenk equilibrium, approximately fit the estimated molar mass of $260 \mathrm{~g} / \mathrm{mol}$. A coexistence of both structures would also be in agreement with lowtemperature NMR experiments which already indicated the presence of a second species in THF solution. The related structure (v) as well as the $\mathrm{MgCl}_{2}$-adduct (vi) are also conceivable, which already hints at the multiplicity of structures possibly present and interconverting in solution. In case of $i \mathrm{PrMgCl} \cdot \mathrm{LiCl}(\mathbf{8})$, the LiCl-adducts (iii) and (iv) have also to be taken into account. The fact that they display higher molecular masses than determined by DOSY experiments might indicate the presence of further species, one of which might be either (ii) and/or (v), in accordance with the performed low temperature ${ }^{1} \mathrm{H}$ NMR experiments. Polynuclear species (e.g. $\mathrm{Mg}_{3}$-species) like those observed in the ESI mass spectrometry investigations are certainly not to be excluded although coordination of THF might rather afford smaller aggregates. They might nevertheless be part of complicated equilibria between increased numbers of different species that cannot be easily resolved by the herein acquired experiments. Therefore, further experiments are required: A titration of $i \mathrm{PrMgCl}(7)$ with a $\mathrm{LiCl}$ solution (9) could allow deeper insight into the behaviour of Turbo Grignard compounds in solution. Furthermore, the second signal set has to be assigned by additional low-temperature experiments, especially low temperature DOSY experiments that might provide information on the molecular size. However, the 
variety of species presumably present in solutions of $i \operatorname{PrMgCl} \cdot \mathrm{LiCl}(\mathbf{8})$ clearly makes an exact analysis and assignment very difficult.

\subsubsection{Anisotropic NMR Experiments}

To perform anisotropic NMR experiments, the sample preparation had to be modified in the way that the polymer sticks were primarily swollen in either toluene- $d_{8}(0.2$ vol\% DVB $)$ or THF- $d_{8}(0.3$ vol\% DVB) followed by subsequent addition of $0.3 \mathrm{~mL}$ of a $0.95 \mathrm{M}$ solution of $i \mathrm{PrMgCl} \cdot \mathrm{LiCl}(\mathbf{8})$ in THF. Employing simultaneous swelling and diffusion to prepare the corresponding gels did not result in any swelling at all despite the similar nature of Grignard and organolithium compounds and the successful preparation of anisotropic organilithium samples by this procedure. A reference sample of $\mathrm{LiCl}$ in PS/ THF- $d_{8}$ (0.2 vol\% DVB) was also prepared. The presence of the particular compound inside the gel was verified by ${ }^{1} \mathrm{H}-\mathrm{CPMG}$ spectra in case of 8 and by ${ }^{7} \mathrm{Li} \mathrm{NMR}$ experiments for $\mathrm{LiCl}(9)$. By ${ }^{2} \mathrm{H}$ NMR experiments the quality of the gels was observed to be only moderate in case of THF displaying a clear splitting of the $\mathrm{CD}_{2}$-groups into two quadrupolar doublets yet with distorted signal shapes. Swelling in toluene- $d_{8}$ afforded samples with higher quality, which is why the corresponding ${ }^{7} \mathrm{Li} \mathrm{NMR}$ spectrum of $\mathbf{8}$ in toluene- $d_{8}$ is depicted on the left of Figure 2.63. Slice-selective NMR experiments were carried out for gels with reduced homogeneity.

iPrMgCl.LiCl (8)
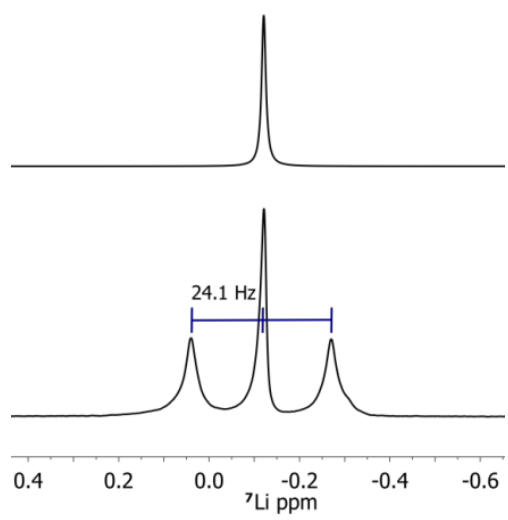

$\operatorname{LiCl}(9)$

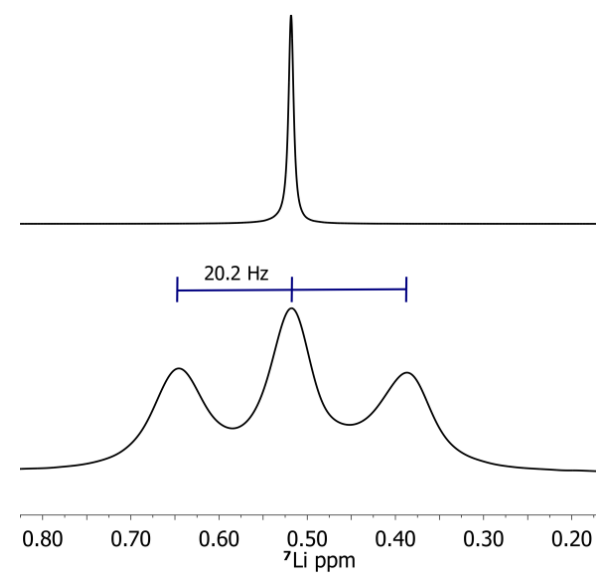

Figure 2.63: Stacked plots of the isotropic and anisotropic ${ }^{7} \mathrm{Li} \mathrm{NMR}$ spectrum of $i \mathrm{PrMgCl} \cdot \mathrm{LiCl}$ (8) (left, PS / toluene- $d_{8}$ and THF from Grignard solution, 0.2 vol\% DVB) and $\mathrm{LiCl}$ (9) on the right (PS / THF- $d_{8}, 0.2$ vol\% DVB) including quadrupolar splitting values.

A quadrupolar splitting in the ${ }^{7} \mathrm{Li}$ NMR spectrum is observed for all three samples with varying splitting values. The quadrupolar splitting constants themselves cannot be easily compared among those experiments due to different solvents and linker concentrations; however the observation of the splitting itself allows drawing some conclusions: A solution 
of $\mathrm{LiCl}$ in $\mathrm{THF}$, which contains dimeric LiCl-aggregates, results in a quadrupolar triplet characteristic for a lithium environment with less than $T_{\mathrm{d}}$ symmetry. The fact, that the anisotropic sample of $\mathbf{8}$ also shows comparable quadrupolar splitting in the ${ }^{7} \mathrm{Li} \mathrm{NMR}$ spectrum thus testifies that the lithium atom is at least temporarily asymmetrically coordinated ruling out the possibility that lithium is exclusively present as the highly symmetrical $\left[\mathrm{Li}(\mathrm{THF})_{4}\right]^{+}$-species postulated by Knochel et al. in the anionic Schlenk equilibrium. ${ }^{[200]}$ Consequently, part of the lithium atoms is either also present as THFsolvated LiCl-dimers or incorporated in Grignard-LiCl-adducts. Both considerations cannot be excluded on the basis of the acquired isotropic NMR measurement even more so as identical values for the ${ }^{7} \mathrm{Li}$ diffusion coefficients were determined for $\mathbf{8}$ and $\mathbf{9}$ and a multiplicity of different aggregates is presumably present in solution. 


\subsubsection{Investigation of ThiLi-Diglyme by NMR Spectroscopy and DFT- Calculations}

\subsubsection{The Structural Motif}

During his study of lithiated thiophenes Markus Granitzka carried out a lithiation reaction in the presence of 1-methoxy-2-(2-methoxyethoxy)ethane, diglyme, according to the following scheme:

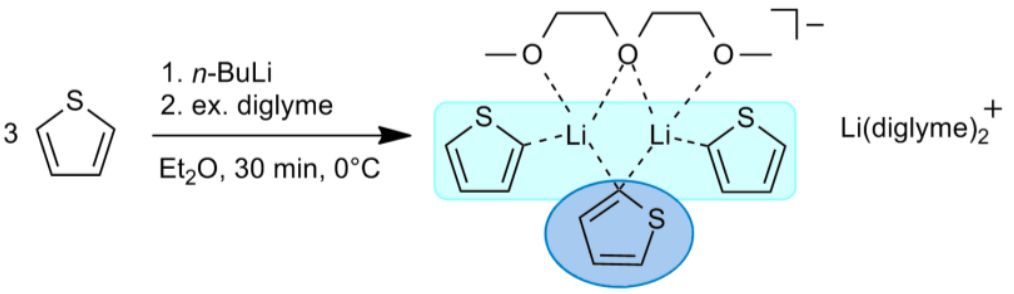

Scheme 2.12: Reaction of thiophene and $n \mathrm{BuLi}$ in the presence of diglyme reproducibly yields crystals containing the solvent separated ion pair $10 .{ }^{[10]}$

Interestingly, he could not observe the formation of a neutral aggregate analogous to the lithiated thiophenes presented in chapter 2.1.2, but instead was able to reproducibly grow crystals of a so called "pentuple ion" (Figure 2.64) as part of a completely solvent-separated ion pair $\left[\mathrm{Li}(\text { diglyme })_{2}\right]\left[(\right.$ diglyme $\left.) \mathrm{Li}_{2}\left(\mathrm{C}_{4} \mathrm{H}_{3} \mathrm{~S}\right)_{3}\right](\mathbf{1 0})$ in the asymmetric unit. Although the discussion of bond lengths and angles was impeded by the high amount of disorder (not depicted in Figure 2.64) the structural motif could be unambiguously derived from X-ray analysis:

$\mathbf{1 0}$ is named "pentuple ion" in analogy to the "triple ion" (see Figure 1.2), because the anionic complex is composed of five charged moieties, namely two lithium cations and three negatively charged organic residues. As illustrated by the colour code in Scheme 2.12, one thiophene moiety (blue) is coordinated by both lithium cations like in dimeric arrangements, whereas the environment of the other two residues (turquoise) corresponds to that of a monomeric motif with only one $\mathrm{Li}-\mathrm{C}_{\alpha}$ contact. The coordination sphere of the lithium atoms is completed by coordination to diglyme which is also present in the counter ion $\left[\mathrm{Li}(\text { diglyme })_{2}\right]^{+}$.
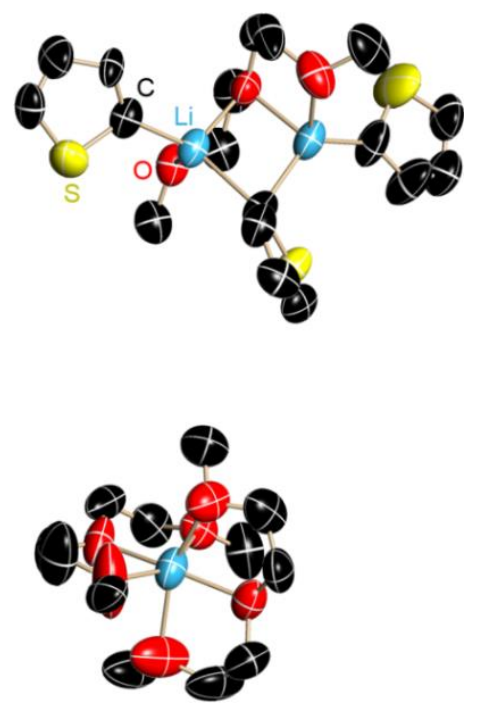

Figure 2.64: Crystal structure of [Li(diglyme $\left.)_{2}\right]\left[(\right.$ diglyme $\left.) \mathrm{Li}_{2}\left(\mathrm{C}_{4} \mathrm{H}_{3} \mathrm{~S}\right)_{3}\right] \quad$ (10) measured by Markus Granitzka. Only the major domain is depicted and hydrogen atoms are omitted for clarity. ${ }^{[10]}$ 
This lithium lithiate represents a kind of "extended" lithiate, e.g. a higher homologue of the triple ion, in which the complex anion consists of three instead of five charged species. ${ }^{[9]}$ While the assembly of five (two positively and three negatively) charged moieties is an unprecedented structural motif in lithiumorganic chemistry heterobimetallic "ate" complexes of a similar assembly are generally confirmed (particularly in magnesiate and zincate compounds where the charges add to zero). ${ }^{[205]}$

\subsubsection{NMR Spectroscopic Studies}

To enable the investigation of the structure in solution, selected crystals of $\mathbf{1 0}$ were directly transferred from the mother liquor into NMR sample tubes. In total, three samples (two with varying concentration in tol- $d_{8}$ and one anisotropic sample (PS/tol- $d_{8}, 0.2 \mathrm{vol} \%$ DVB)) were prepared. All samples were sealed and stored at low temperature due to the overall instability of 10, which also impeded washing of the crystals prior to dissolving them in toluene. In agreement with the results presented in chapter 2.2.2, swelling of the anisotropic sample was successful at $4{ }^{\circ} \mathrm{C}$. First, conventional one- and two-dimensional NMR experiments were carried out, followed by DOSY and ${ }^{1} \mathrm{H},{ }^{7} \mathrm{Li}-\mathrm{HOESY}$ experiments to obtain a deeper understanding according to the procedure described above for the other 2thienyllithium derivatives (chapter 2.1.2).

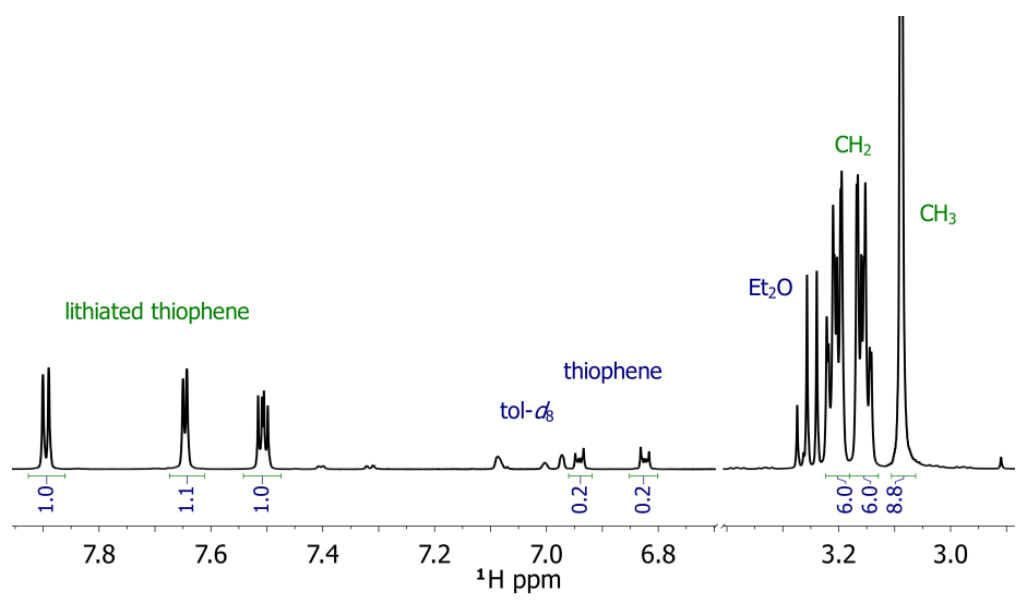

Figure 2.65: ${ }^{1} \mathrm{H}$ NMR spectrum of 10 in toluene- $d_{8}(298 \mathrm{~K})$, including integrals. Only minor impurities $\left(\mathrm{Et}_{2} \mathrm{O}\right.$, thiophene) can be detected. A small excess of diglyme is observed.

In agreement with a fast equilibrium between diglyme molecules of the (separated) cation and diglyme molecules adjacent to the thiophene moieties, only one set of signals is observed for a solution of $\mathbf{1 0}$ in toluene- $d_{8}$ at room temperature (Figure 2.65). Furthermore, the measured integrals directly match the employed quantities of the reactants, namely a 1:1 ratio of thiophene and $n \mathrm{BuLi}$ with a slight excess of diglyme. The ${ }^{7} \mathrm{Li}$ NMR spectrum also displays only one signal at room temperature. However, the $\mathrm{C}_{\alpha^{-}}$ chemical shift which turned out to be highly indicative of the aggregation state for the 
other thiophene derivatives, is at $172.5 \mathrm{ppm}$ which would fit very well to a dimeric arrangement, e.g. as in $\left[(\mathrm{DME}) \mathrm{Li}\left(\mathrm{C}_{4} \mathrm{H}_{3} \mathrm{~S}\right)\right]_{2}(\mathbf{2 c}, 172.1 \mathrm{ppm})$ and $\left[(\mathrm{TMEDA}) \mathrm{Li}\left(\mathrm{C}_{4} \mathrm{H}_{3} \mathrm{~S}\right)\right]_{2}(\mathbf{2 d}$, $174.4 \mathrm{ppm}$ ). This might already hint at a change of aggregation upon dissolving in toluene. The current SSIP might recombine to one dimeric and two monomeric species or averaging of the coordination motifs within $\mathbf{1 0}$ might also cause this particular chemical shift. ${ }^{1} \mathrm{H}$ and ${ }^{7} \mathrm{Li}$ diffusion (DOSY) experiments did not promote the characterization in solution or further illuminated the aggregation behaviour of 10. Since the ${ }^{1} \mathrm{H},{ }^{7} \mathrm{Li}-\mathrm{HOESY}$ experiment already proved very valuable for the study of the above presented 2thienyllithium aggregates (see 2.1.2), this experiments was also employed herein with the aim to identify the aggregation state in solution. A representative ${ }^{1} \mathrm{H},{ }^{7} \mathrm{Li}-\mathrm{HOESY}$ spectrum is depicted at the left of Figure 2.66.
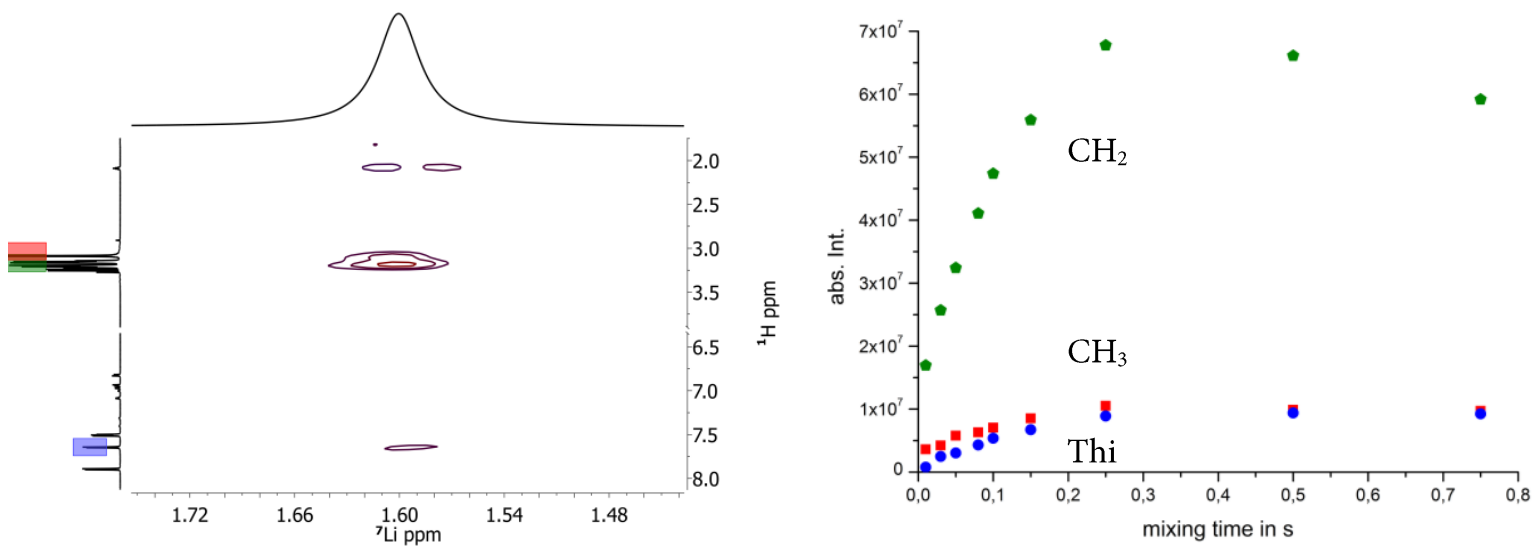

Figure 2.66: ${ }^{1} \mathrm{H},{ }^{7} \mathrm{Li}-\mathrm{HOESY}$ spectrum of 10 in toluene- $d_{8}(298 \mathrm{~K})$ recorded with a mixing time of $0.5 \mathrm{~s}$ (left) and build-up curve of the ${ }^{1} \mathrm{H},{ }^{7} \mathrm{Li}-\mathrm{HOE}$ in $\left[\mathrm{Li}(\text { diglyme })_{2}\right]\left[(\right.$ diglyme $\left.) \mathrm{Li}_{2}\left(\mathrm{C}_{4} \mathrm{H}_{3} \mathrm{~S}\right)_{3}\right](\mathbf{1 0})$ in toluene- $d_{8}$ (right) with mixing times varied between 0.01 and $0.8 \mathrm{~s}$. Cross peaks could be observed for the neighbouring thiophene proton (๑) and the $\mathrm{CH}_{2-}(\bullet) / \mathrm{CH}_{3}$-groups ( $\bullet$ ) of diglyme.

As clearly visible in this spectrum, strong cross peaks of the $\mathrm{CH}_{2}$ and $\mathrm{CH}_{3}$ groups of the diglyme moiety heavily overlap impeding a clear integration of both signals. Therefore, the integration was achieved in a one dimensional slice extracted from the full twodimensional spectrum. In total, nine ${ }^{1} \mathrm{H},{ }^{7} \mathrm{Li}-\mathrm{HOESY}$ spectra with mixing times varied between 0.01 and $0.8 \mathrm{~s}$ were recorded, and the plot of the extracted absolute integrals as a function of the mixing time resulted in a typical HOE build-up (Figure 2.66, right). Accordingly, the slope of the build-up at short mixing times were extracted, and comparison with the calculated $\Sigma r(\mathrm{H} \cdots \mathrm{Li})^{-6}$ terms from the crystal data of $\mathbf{1 0}$ yielded the graph depicted in Figure 2.67. 


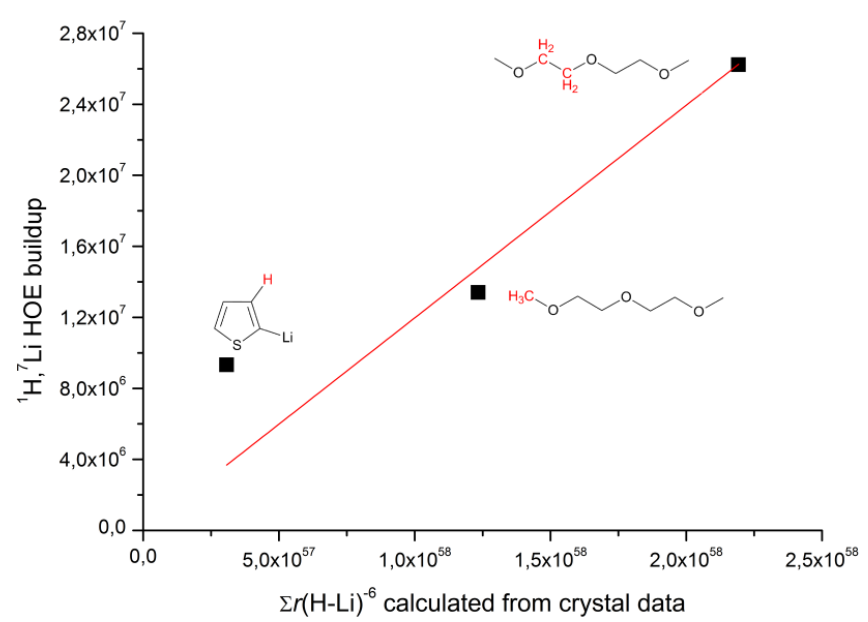

Figure 2.67: Comparison of the ${ }^{1} \mathrm{H},{ }^{7} \mathrm{Li}-\mathrm{HOE}$ build-up with the calculated $\Sigma r(\mathrm{H} \cdots \mathrm{Li})^{-6}$ terms from crystal data of 10. Disorder in the crystal structure has been taken into account for these calculations.

The correlation looks promising, yet, compared to the diglyme data points the thiophene contacts seem slightly underrepresented by the distances derived from the crystal data. This would indicate a higher aggregation. Furthermore, this is in contrast to the structures analysed in chapter 2.1.2, where the ligand contacts rather than the thiophene distances were overestimated by the crystal structure.

The preparation of an anisotropic NMR sample was also successful, and after swelling for one week at $4{ }^{\circ} \mathrm{C}$ the PS / tol- $d_{8}$ sample was sufficiently homogeneous to perform ${ }^{7} \mathrm{Li} \mathrm{NMR}$ experiments and extract RDCs $(3 \mathrm{x}$ for the $\mathrm{CH}$ groups of the thiophene moiety, $2 \mathrm{x}$ for the different $\mathrm{CH}_{2}$ units of the diglyme, $1 \mathrm{x}$ for the $\mathrm{CH}_{3}$ groups of the diglyme molecule and $1 \mathrm{x}$ $\mathrm{HH})$. A comparison of the isotropic and anisotropic ${ }^{7} \mathrm{Li}$ NMR spectra of $\mathbf{1 0}$ is depicted in Figure 2.68. A quadrupolar splitting of $46 \mathrm{~Hz}$ is observed for the partially aligned sample at room temperature in accordance with a low-symmetry aggregate (less than $T_{\mathrm{d}}$ ). The RDCs measured at ambient temperature were correlated to the crystal structure but unfortunately showed very poor agreement.

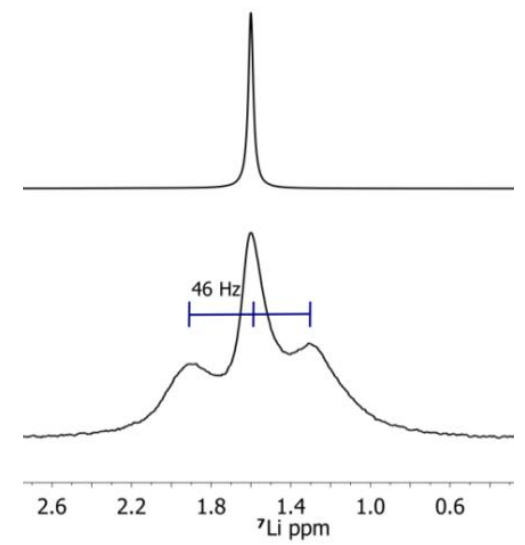

Figure 2.68: Isotropic (top) and anisotropic (bottom) ${ }^{7} \mathrm{Li}$ NMR spectrum of 10 in toluene- $d_{8}$ and PS / tol- $d_{8}$, respectively. 
To gain deeper insight into the aggregation behaviour of this complex and to verify the postulated exchange between the cation and complex anion, low-temperature NMR experiments were performed at $0,-25,-50$ and $-80^{\circ} \mathrm{C}$. Over the whole temperature range only one signal set with some broadening towards the low-temperature end was observable in the ${ }^{1} \mathrm{H}$ NMR spectrum. In the ${ }^{7} \mathrm{Li}$ NMR spectrum, however, a splitting of the initial singlet into at least three distinct peaks with significantly different line widths became visible below $-50{ }^{\circ} \mathrm{C}$ (Figure 2.69).

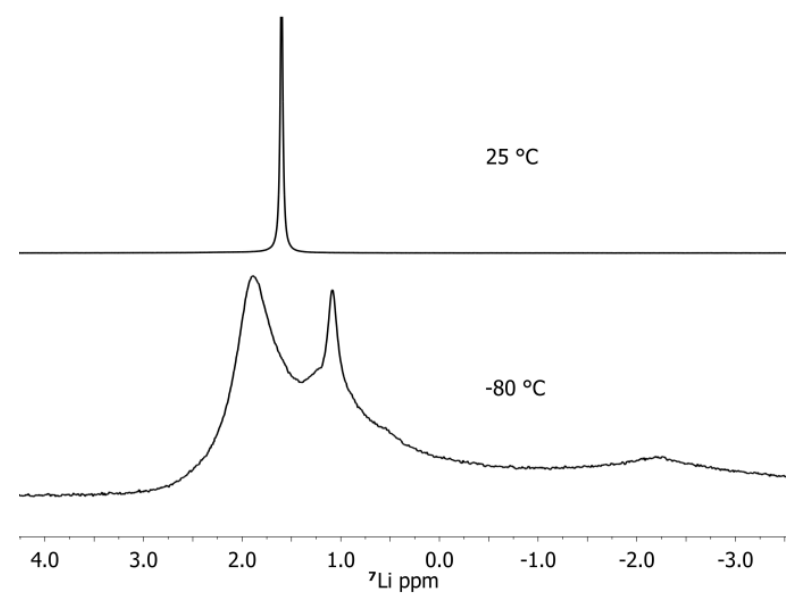

Figure 2.69: Low temperature (top) and room temperature (bottom) ${ }^{7} \mathrm{Li}$ NMR spectrum of 10 in toluene- $d_{8}$.

In consideration of the crystal structure, the presence of two separate lithium environments seems justifiable, that is to say one signal representing the cationic lithium atom and one arising from the lithium inside the complex anion. Hence, the presence of three lithium signals points towards additional or different species interconverting in solution. The rearrangement of similar lithium lithiates in the solid state and in solution is not unknown ${ }^{[206]}$ just as the observation of broad lines for complex anions due to lower symmetry. ${ }^{[207]}$ The latter might allow a tentative assignment of the cation to the narrowest and the complex anion to one of the broader lithium signals, however, the broad line shape impedes an auxiliary correlation by ${ }^{1} \mathrm{H},{ }^{7} \mathrm{Li}$-HOESY experiments. Although a cross peak towards the diglyme moiety can be detected for the signals at 1 and $2 \mathrm{ppm}$ no other contacts whatsoever can be extracted from the spectrum.

Nevertheless, the low temperature study might give an explanation for the slight deviation observed in the HOE - distance comparison resulting from a third species in solution. The entity of the acquired NMR experiments therefore allows the hypothesis of an equilibrium between the lithiate structure partially retained upon dissolving in toluene and an additional aggregate that also contains coordinated diglyme units. The later became visible in a low-temperature ${ }^{1} \mathrm{H},{ }^{7} \mathrm{Li}-\mathrm{HOESY}$ spectrum. However, additional information by means of anisotropic NMR is not realizable, because of the low-temperature limit of about $-40{ }^{\circ} \mathrm{C}$ 
for the observation of quadrupolar parameters. Solid state NMR spectroscopy as complementary source of structural information was also not feasible due to the fast decomposition of the crystalline material upon drying. This is why Density Functional Theory (DFT) calculations were performed to yield a clarification of the behaviour of $\mathbf{1 0}$ in solution on a theoretical basis. All calculations were performed in cooperation with Jun.Prof. Dr. Ricardo Mata from the Physical Chemistry Department at this Faculty.

\subsubsection{Preliminary DFT Calculations}

In a first step, structural motifs conceivable for 2-thienyllithium.diglyme were constructed on the basis of the above-mentioned X-ray and NMR data (Scheme 2.13).

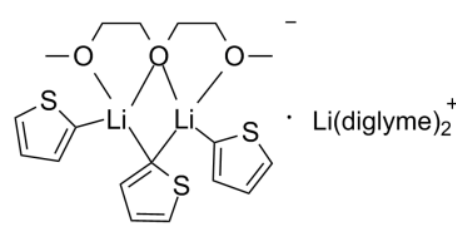

crystal structure

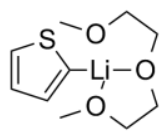

monomer i) and ii)

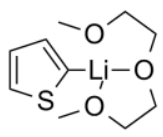

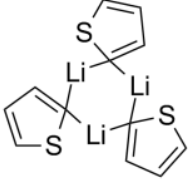

trimer diglyme omitted

Scheme 2.13: Overview over possible structural motifs of 2-thienyllithium.diglyme.

Apart from the crystal structure, the formation of monomeric, dimeric and trimeric units seemed plausible. Furthermore, only aggregates with a 1:1:1 ratio of thiophene, lithium and diglyme were taken into consideration. The common disorder of the thienyl moiety was accounted for by an additional structure with a flipped ring, exemplarily only depicted for the least crowded monomeric structure (Scheme 2.13, i and ii). Formation of an additional "ate" species, i.e. a triple ion, seemed not feasible due to the low steric demand of the thienyl moieties. Aggregates with higher symmetry were discarded owing to the previously described anisotropic NMR experiments that only allow the presence of aggregates with lower than $T_{\mathrm{d}}$ symmetry.

For a first overview, the idea was to optimize these geometries at a moderate level of theory along with an evaluation of the reaction energies of plausible interconversions between the different aggregates (Scheme 2.14). This would then yield a differentiation between thermodynamically favoured or disfavoured aggregation motifs. The favoured species would later on be treated at a higher level of theory including solvent effects. 


\begin{tabular}{|c|c|c|c|c|c|c|c|}
\hline LiDS & + & LiDS & $\rightarrow$ & $\mathbf{L i}_{2} \mathrm{D}_{2} \mathrm{~S}_{2}$ & & & (1) \\
\hline & & $3 \mathrm{Li}_{2} \mathrm{D}_{2} \mathrm{~S}_{2}$ & $\rightarrow$ & $2 \mathrm{Li}_{2} \mathrm{DS}_{3}^{-}$ & + & $2 \mathrm{LiD}_{2}^{+}$ & (2a) \\
\hline & & 3 LiDS & $\rightarrow$ & $\mathrm{Li}_{2} \mathrm{DS}_{3}^{-}$ & + & $\mathrm{LiD}_{2}^{+}$ & (2b) \\
\hline $\mathrm{Li}_{2} \mathrm{D}_{2} \mathrm{~S}_{2}$ & + & LiDS & $\rightarrow$ & $\mathbf{L i}_{2} \mathrm{DS}_{3}^{-}$ & + & $\mathrm{LiD}_{2}^{+}$ & (2c) \\
\hline \multirow[t]{2}{*}{$\mathrm{Li}_{2} \mathrm{DS}_{3}^{-}$} & + & $\mathrm{LiD}_{2}^{+}$ & $\rightarrow$ & $\mathrm{Li}_{3} \mathrm{D}_{3} \mathrm{~S}_{3}$ & & & (3a) \\
\hline & & $3 \mathrm{Li}_{2} \mathrm{D}_{2} \mathrm{~S}_{2}$ & $\rightarrow$ & $2 \mathrm{Li}_{3} \mathrm{D}_{3} \mathrm{~S}_{3}$ & & & $(3 b)$ \\
\hline $\mathrm{Li}_{2} \mathrm{D}_{2} \mathrm{~S}_{2}$ & + & LiDS & $\rightarrow$ & $\mathrm{Li}_{3} \mathrm{D}_{3} \mathrm{~S}_{3}$ & & & (3c) \\
\hline
\end{tabular}

Scheme 2.14: Schematic representation of possible reactions between 2-thienyllithium.diglyme aggregates in solution. The following abbreviations are used: $\mathrm{LiDS}$ monomer, $\mathrm{Li}_{2} \mathrm{D}_{2} \mathrm{~S}_{2}$ dimer, $\mathrm{Li}_{3} \mathrm{D}_{3} \mathrm{~S}_{3}$ trimer and the crystal structure $\left[\mathrm{Li}_{2} \mathrm{DS}_{3}\right]\left[\mathrm{LiD}_{2}\right]$.

The initial geometry optimizations were carried out in the gas phase at the BP86-D3/def2SVP level of theory ${ }^{[208]}$ with the ORCA program package, ${ }^{[208 e]}$ which requires less calculation time, thus allowing a more thorough sampling of the studied system. After geometry optimization, frequency calculations were carried out to confirm the calculated structures to be true minima on the potential energy surface and to obtain thermodynamic data. All calculated frequencies were real, except for the $\left[\mathrm{Li}(\text { diglyme })_{2}\right]^{+}$-cation, which suffers from movements of the methyl groups. This effect can be attributed to basis set superposition effects, which is to be expected given the small basis set size. Such effects can be observed, for example, in acetone. ${ }^{[209]}$ A summary of the resulting molecular structures is depicted in Figure 2.70. Separate geometry optimization of the two ionic species of which the crystal structure is composed resulted in the stable structures displayed on the left. The monomeric and dimeric form could also be verified by these calculations. Thereof, the rotamer with the lowest energy (either i) or ii) from Scheme 2.13), was chosen for further investigations. In contrast, no stable refinement was achieved for the trimeric structure, which always resulted in a dimeric structural motif while the remaining fragments were supplanted to the outer sphere of the aggregate. Additionally, this aggregate is also disfavoured from the entropic point of view. Therefore, the presence of trimers and thus also the interconversions $\mathbf{3 a}$-c were excluded from further investigations. 


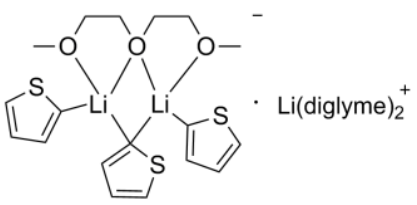

crystal structure

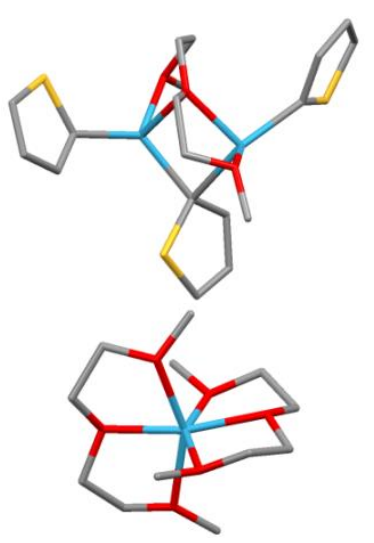

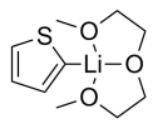

monomer

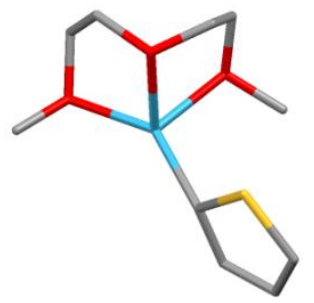

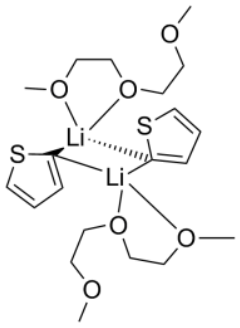

dimer

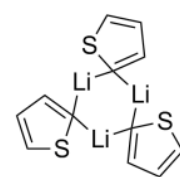

trimer

diglyme omitted

Figure 2.70: Optimized geometries for selected structures of 2-thienyllithium.diglyme at the BP86-D3/def2SVP level of theory. The two ions of the crystal structure were optimized separately and geometry optimization of the trimer did not yield any stable structure. Hydrogen atoms are omitted for clarity.

The free energies of the resulting optimized structures were obtained and used to calculate the free reaction energies according to Scheme 2.14 (Figure 2.71). Interestingly, only the formation of dimers $\mathrm{Li}_{2} \mathrm{D}_{2} \mathrm{~S}_{2}$ from monomeric 2-thienyllithium moieties $\operatorname{LiDS}(\mathbf{1})$ is thermodynamically favoured in the gas phase while every interconversion into the ionic crystal structure $\left[\mathrm{Li}_{2} \mathrm{DS}_{3}\right]\left[\mathrm{LiD}_{2}\right](\mathbf{2 a - c})$ is not feasible at this level of calculation (blue bars in Figure 2.71).

To account for solvation effects, all species except the trimer were recomputed with the COSMO model. ${ }^{[210]}$ Based on the fact that all NMR experiments were carried out in toluene- $d_{8}$, a dielectric constant of $\varepsilon=2.4$ (toluene at ambient temperature) was provided to the program. The free energies of reaction were obtained analogously and the relevant interconversions in solution are represented by the green bars in Figure 2.71. Despite the low polarity of toluene compared to, for instance, THF $(\varepsilon=7.6)$ or water $(\varepsilon=80)$, it has a substantial impact on the relative stabilisation of the aggregation motifs. While in the gas phase only dimeric species were found to be thermodynamically stable, the solvent contribution relatively stabilises the ionic form, thus making reaction $\mathbf{2 b}$ thermodynamically favourable. 


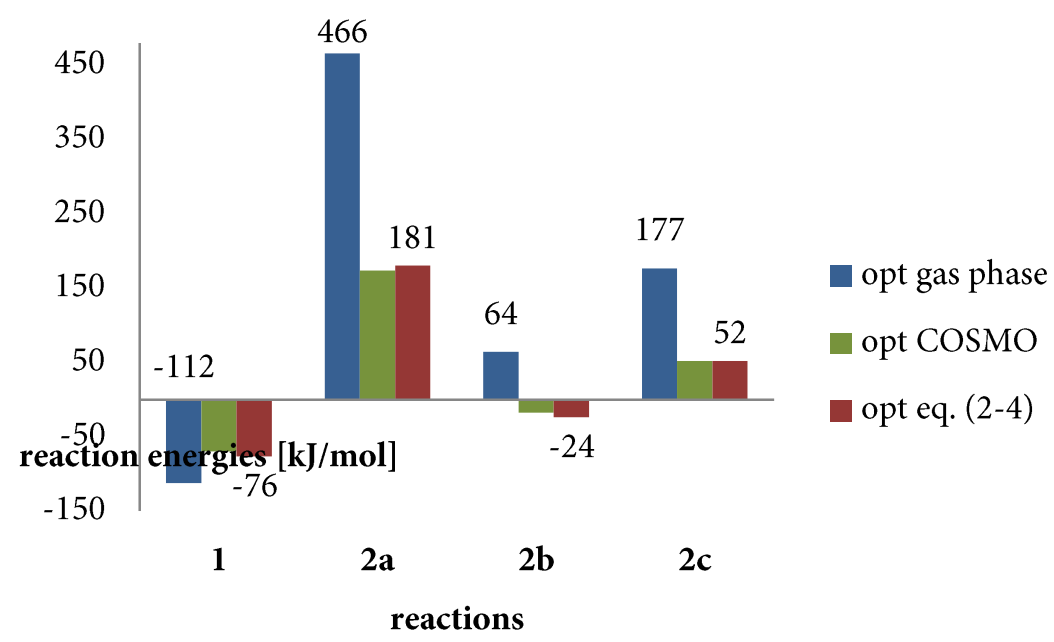

Figure 2.71: Free energies of possible interconversions of 2-thienyllithium-diglyme after optimization at the BP86-D3/def2-SVP level of theory. Complete gas phase calculations are represented in blue bars, solvent effects included through the COSMO model by green bars. Red bars indicate energies obtained by a combination of gas phase and COSMO calculations. The numbering of the reaction refers to Scheme 2.14.

The red bars are energy values obtained for a calculation according to the following equation (2-4):

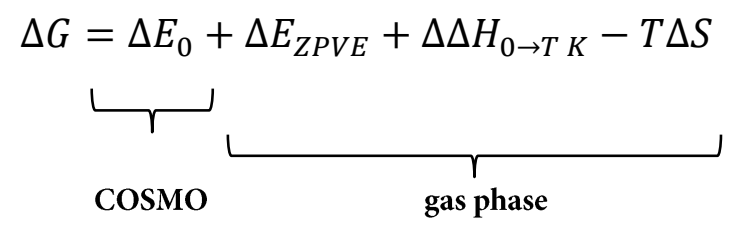

Here, the free energy is described by different contributions: the total electronic energy $E_{0}$, the zero-point vibrational energy $E_{Z V P E}$ along with a temperature correction term to the enthalpy $\Delta \Delta H_{0 \rightarrow} \rightarrow_{K}$ and the entropy term $T \Delta S$. While the electronic energy is derived from a COSMO refinement, the structure and the correction terms are obtained by gas phase calculations. The corresponding values show a small deviation yet the overall tendency is retained. Therefore, this approach might be adapted to facilitate the introduction of different solvent effects into the model. In the following, only the lowest minima of the preliminary geometry optimization are taken into account and reoptimized at a higher level of theory to obtain (i) more precise geometries that are finally compared with ${ }^{1} \mathrm{H},{ }^{7} \mathrm{Li}$ HOESY data and (ii) more precise energy values that allow a final evaluation of the different interconversions. In order to separately implement particular conditions like impurities of diethyl ether (see Figure 2.68) a combined approach similar to the one in equation (2-4) was chosen. 


\subsubsection{Final Structure Refinement}

At the B3LYP-D3/def2-TZVP level of theory ${ }^{[208 a, 208,211]}$ a final refinement was carried out by Ricardo Mata which resulted in the following optimized structures (Figure 2.72).

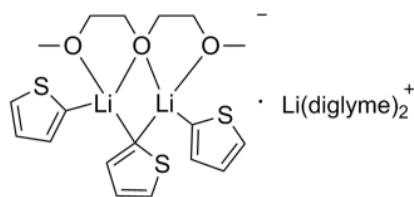

crystal structure

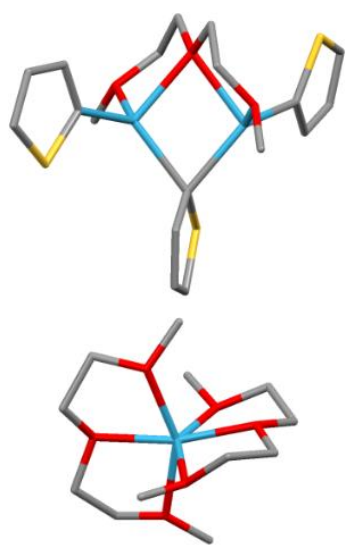

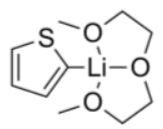

monomer

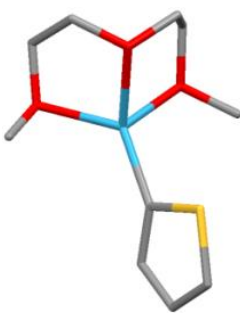

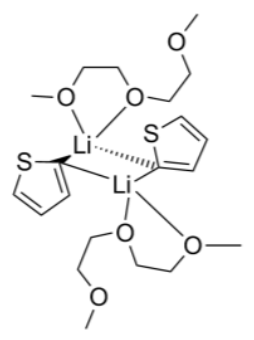

dimer

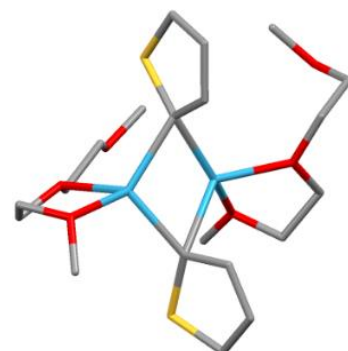

Figure 2.72: Final structure refinement for the selected structures of 2-thienyllithium.diglyme at the B3LYPD3/def2-TZVP level of theory. The two species that exist in the crystal structure were optimized separately and hydrogen atoms are omitted for clarity.

At this level of theory the observed geometries are considerably more symmetric, and especially the monomeric structure seems to be a more realistic representation (e.g. arrangement of the thiophene moiety and the lithium atom, see Figure 2.70).

To judge on the aggregation behaviour of 10, different approaches were chosen for the energy refinement. In a first step, the geometries were optimized at the B3LYP-D3/def2TZVP level of theory including solvent effects in toluene $(\varepsilon=2.4)$ followed by a frequency calculation to obtain Gibbs energies for the particular aggregates. After this complete refinement process, the contributions of $\Delta G$ were split similar to the approach described in the preceding chapter in order to straightforwardly account for a different solvent environment of the aggregates.

The electronic energy is therein calculated by different methods (B3LYP-D3, $\operatorname{LCCSD}(\mathrm{T} 0)^{[212]}$ ) and by applying different conditions (gas phase, toluene, $\mathrm{Et}_{2} \mathrm{O}$ ), while the energy correction term is supposed not to change dramatically when varying the only moderately interacting solvent system (low $\varepsilon$ values). Therefore, the values derived from the above-described optimization in the presence of toluene as solvent are employed in this 
analysis. Although this approach represents an approximation of the existing conditions and thus yields less precise absolute energy values, it does clearly not impede the recognition of general trends upon modification of the solvent environment. A comparison of the reaction energies for interconversions $\mathbf{1}$ and $\mathbf{2 a - c}$, calculated analogously to those presented in chapter 2.3.2.3, is given by the following figure (Figure 2.73). Density-fitting approximations were used with $\operatorname{LCCSD}(\mathrm{T} 0)^{[212]}$. The basis set applied in the latter calculations was $c c-p V T Z^{[213]}$ (cc-pCVTZ ${ }^{[214]}$ for $\mathrm{Li}$, correlating the 1 s electrons). The density fitting bases were cc-pVTZ/JKFIT ${ }^{[215]}$ (def2-TVPP/JKFIT ${ }^{[216]}$ for Li) and ccpVTZ/MP2FIT $^{[217]}$ (cc-pwCVTZ/MP2FIT ${ }^{[218]}$ for Li). The B3LYP-D3 single point energies were obtained with the same basis set used for optimizations (def2-TZVP).

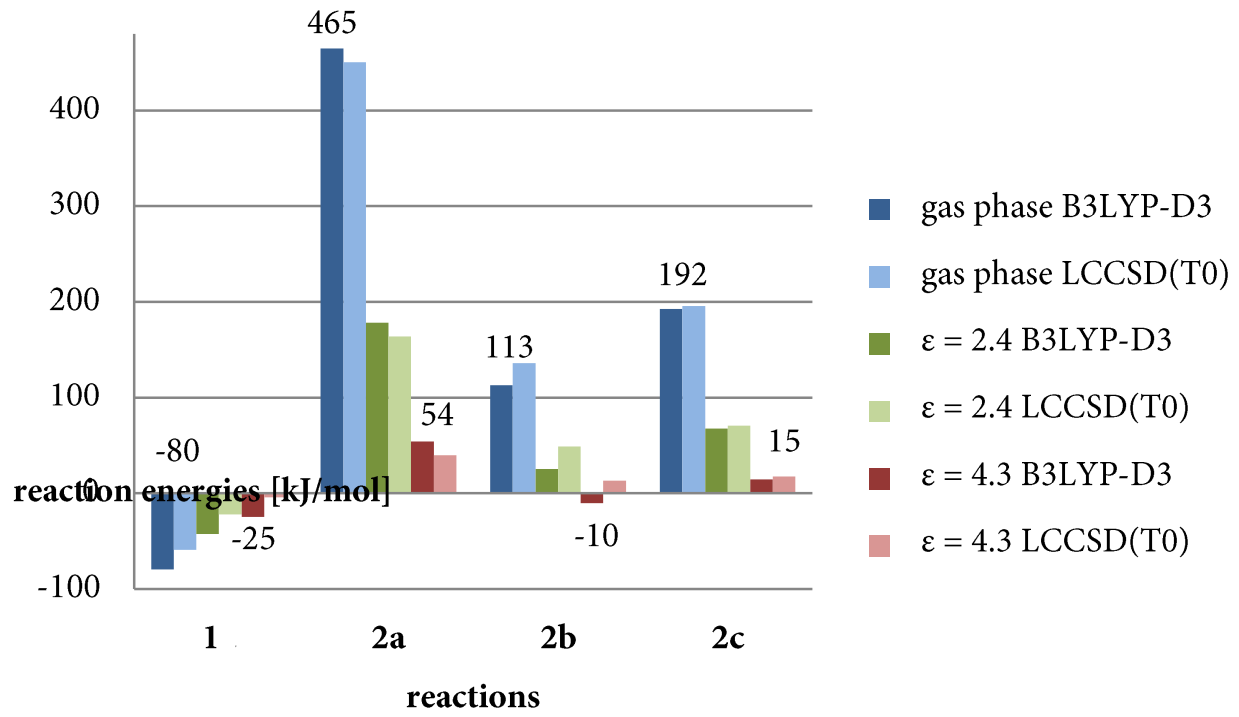

Figure 2.73: Reaction energies of interconverions 1 and 2a-c for the final structure refinement. For each reaction, B3LYP-D3 and Local Coupled-Cluster theory with Single and Double excitations including noniterative perturbative triples ( $\operatorname{LCCSD}(\mathrm{T} 0)$ ) approaches were combined with COSMO corrections for the solvents environments $\varepsilon=2.4$ (toluene) and $\varepsilon=4.3\left(\mathrm{Et}_{2} \mathrm{O}\right)$.

Therein, $\mathbf{1}$ is the formation of a dimeric unit out of two monomers, while reactions $\mathbf{2 a - c}$ represent the construction of the lithium lithiate structure starting from both monomeric and/or dimeric units. In the following, it will be abstained from interpreting energy values directly for all environments. Instead, the focus will be put on the discussion of general trends.

From the gas phase via the toluene solution to the diethyl ether solution, the formation of a 2-thienyllithium dimer is thermodynamically favoured. However, with increasing polarity, this neutral aggregate having the smallest effective dipole moment is relatively destabilized. In contrast, all reactions towards the ion pair structure (2a-c) are stabilized in increasingly polar environment. For reaction $\mathbf{2 b}$, the formation of $\mathbf{1 0}$ due to rearrangement of three 
monomeric units, even a very small negative reaction energy is observed for the B3LYP approach in diethyl ether despite the entropy. This relative destabilisation of the neutral aggregates in favour of charged species upon going from gas phase to an ethyl ether solvent environment is in very good agreement with both the crystallization attempts and the NMR analysis in solution, because crystallization was done from an $\mathrm{Et}_{2} \mathrm{O}$ solution. However, the obtained reaction energies are not as negative as to keep the solvated molecules in a thermodynamic sink. Instead, diethyl ether seems to be a very potent solvent for crystallization approaches, which theoretically underlines the large number of 2thienyllithium aggregates obtained by crystallization from $\mathrm{Et}_{2} \mathrm{O}$. Upon dissolving crystals of 10 in toluene- $d_{8}$ dimers should be predominantly formed according to the calculations presented above. The presence of $\mathrm{Et}_{2} \mathrm{O}$ due to the transfer of the crystals from mother liquor stabilises also the existence of the charged lithium lithiate structure. This is in accordance with the ${ }^{13} \mathrm{C}$ chemical shift in the dimeric region at the same time explaining the observation of three signals in the ${ }^{7} \mathrm{Li}$ NMR spectrum recorded at low temperatures. Evaluation of the ${ }^{1} \mathrm{H},{ }^{7} \mathrm{Li}-\mathrm{HOESY}$ data clearly shows, that the HOE build-up is best described by coexisting dimers and lithium lithiates instead of one single species (Figure 2.74).

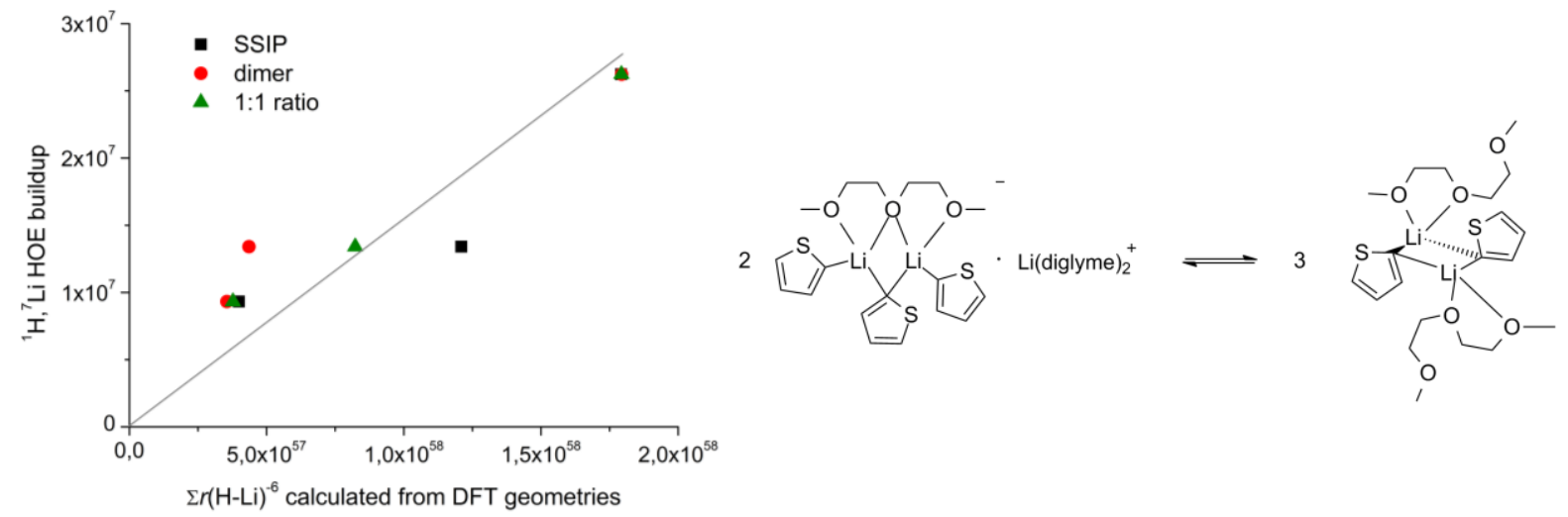

Figure 2.74: Comparison of the ${ }^{1} \mathrm{H},{ }^{7} \mathrm{Li}-\mathrm{HOE}$ build-up with the calculated $\Sigma r(\mathrm{H} \cdots \mathrm{Li})^{-6}$ terms for the SSIP (crystal structure), the dimer and a 1:1 ratio of both, obtained from minimum energy structures of 2thienyllithium-diglyme at the B3LYP-D3/def2-TZVP level of theory (left). Proposed aggregation behaviour of 10 in solution (right).

To conclude, the combination of X-ray diffraction, NMR spectroscopy and DFT calculations proved to be very fruitful enabling to explain the formation of the crystal structure form diethyl ether and gain detailed insight into the aggregation behaviour in the gas phase as well as in toluene and diethyl ether solution. 


\section{SUMMARY AND OUTLOOK}

In summary, this thesis is divided into three main parts, namely: structure elucidation by (i) isotropic NMR spectroscopy, (ii) by application of anisotropic NMR spectroscopy in polymer gels and (iii) by a combination of the aforementioned techniques with ESI mass spectrometry or DFT calculations.

Concerning the characterization of organometallic compounds in isotropic solution, modern 2D NMR experiments at variable temperatures were acquired. Based on the knowledge of crystal structure analyses, the aggregation behaviour of two systems, $[t \mathrm{BuLi}]_{4} \cdot 4\left[\mathrm{Me}_{2} \mathrm{NC}_{6} \mathrm{H}_{4} \mathrm{Li}\right]_{4}$ and 2-thienyllithium, was investigated. The cocrystallized compound $[t \mathrm{BuLi}]_{4} \cdot 4\left[\mathrm{Me}_{2} \mathrm{NC}_{6} \mathrm{H}_{4} \mathrm{Li}\right]_{4}$ was successfully characterized by means of ${ }^{1} \mathrm{H}$ - and ${ }^{7} \mathrm{Li}$ - diffusion-ordered spectroscopy (DOSY) as well as exchange spectroscopy (EXSY) supporting the presence of five exchanging species: homotetramers $\mathbf{A}$ and $\mathbf{E}$ and heterotetramers B-D resulting from consecutive substitution of ortho-lithiated DMA moieties by $t$ BuLi units.

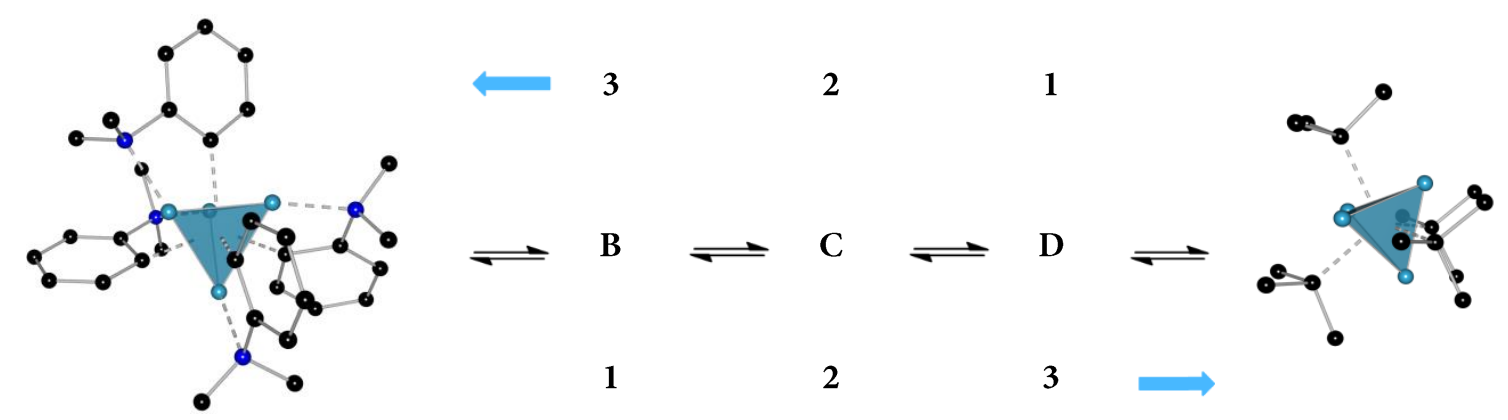

Figure 3.1: Illustration of the five exchanging species of $[t \mathrm{BuLi}]_{4} \cdot 4\left[\mathrm{Me}_{2} \mathrm{NC}_{6} \mathrm{H}_{4} \mathrm{Li}\right]_{4}$ in solution.

Full assignment $\left({ }^{1} \mathrm{H},{ }^{13} \mathrm{C}\right.$ and ${ }^{7} \mathrm{Li}$ resonances) of all species was also achieved. Contrarily to this dynamic behaviour in solution at variance with the solid compound, the structure of differently coordinated 2-thienyllithium aggregates was shown to be predominantly retained upon dissolving the solid material in toluene- $d_{8}$. Application of ${ }^{1} \mathrm{H},{ }^{7} \mathrm{Li}-\mathrm{HOESY}$ experiments was most expedient for the extraction of distance information that was subsequently compared with distances in the crystal structures. Even a correlation of the structural features with the displayed reactivity, which turned out to be highest for 2thienyllithium coordinated by nitrogen Lewis bases, was possible. These two aggregation studies on the one hand unambiguously underline the importance of advanced 2D NMR experiments for the investigation of organolithium compounds and on the other hand 
prove the bridge between crystal structure analysis in solid state and NMR in solution to be eminently important for a comprehensive characterization of these compounds. The rich diversity of 2D NMR experiments gives broad access to various structural and dynamical aspects of aggregation, bonding and coordination, which substantially contributes to a thorough understanding of metal complexes.

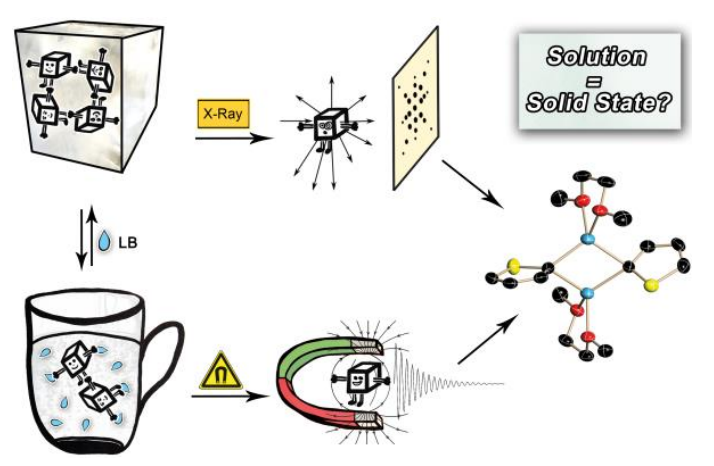

Figure 3.2: Visualization of the work on 2-thienyllithium (courtesy of Roman Neufeld).

In the second part, the employment of anisotropic NMR for structure elucidation in solution is described. This is the first example of the application of strain induced alignment in a gel (SAG) to highly reactive organometallic compounds. After small modifications to a literature-known polymerization protocol, polystyrene gels swollen in toluene- $d_{8}$, withstood the very harsh conditions associated with compounds like monomeric 2-thienyllithium.PMDETA. These samples were stable for at least four weeks and enabled a detailed extraction of anisotropic NMR parameters.

${ }^{7} \mathrm{Li}$ residual quadrupolar couplings (RQCs) proved to be a very valuable tool for the analysis of the aggregation state of organolithiums. By measurement of only two ${ }^{7} \mathrm{Li} \mathrm{NMR}$ spectra (one in anisotropic environment and one of an isotropic reference sample) a straightforward discrimination between high- and low- symmetry aggregates can be afforded at very much reduced measurement times. While tetrameric or hexameric aggregates preserve a singlet in the anisotropic ${ }^{7} \mathrm{Li}$ NMR spectrum, dimeric or monomeric species yield a quadrupolar triplet. The method is also applicable at low temperatures thus allowing also the distinction of species involved in exchange equilibria. It was clearly demonstrated how versatile SAG is for structure elucidation of organolithium compounds. A next step in the interpretation of ${ }^{7} \mathrm{Li}$ RQCs might be envisaged by charge density studies with the aim to determine the efg-tensor on the basis of point charges of the surrounding direct neighbours. Future work with respect to the RDC method would also need to focus on the reduction of the preparation time of the anisotropic samples along with augmenting the overall homogeneity of the samples. Drawing attention to synthetic organometallic chemistry, a possible application of SAG may be the addressing of more complex lithiation 
reactions. Broadening the scope of RQCs to other quadrupolar nuclei might be very versatile in many different fields of chemistry ranging from frustrated Lewis pairs to carbenes, zincates and cuprates, only to mention a few.
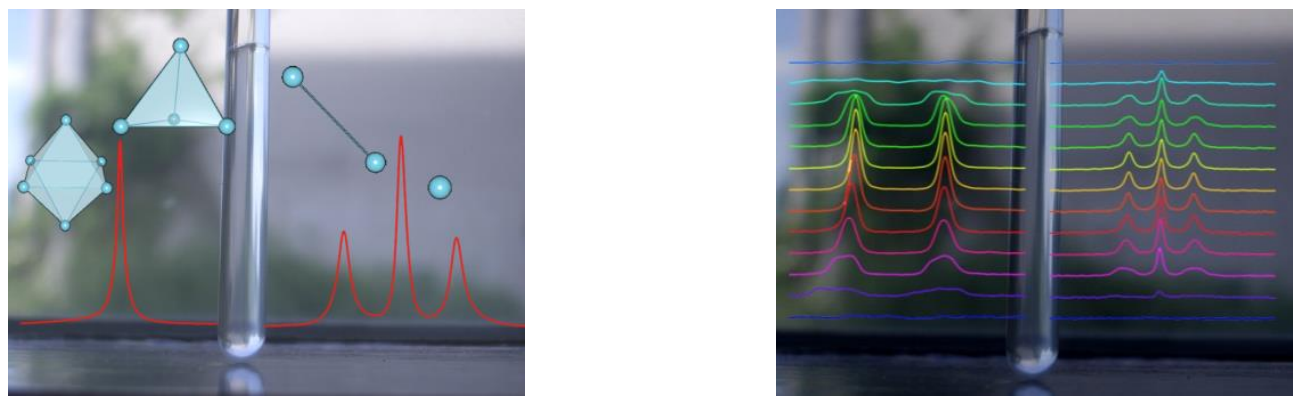

Figure 3.3: Illustration on how to discriminate between different degrees of aggregation in lithiumorganic chemistry by measurement of ${ }^{7} \mathrm{Li}$ RQCs (left) and typical spectra obtained by ${ }^{2} \mathrm{H}$ and ${ }^{7} \mathrm{Li}$ slice-selective NMR experiments.

Since the homogeneity of the polymer gel is a vital requirement for the SAG method, sliceselective excitation (SSE) was employed affording a way to sample the homogeneity of both composition and strain at distinct positions of the polymer gel. Swelling with lithium hexamethyldisilazane (LiHMDS) in toluene- $d_{8}$ allows monitoring the diffusion of both solvent $\left({ }^{2} \mathrm{H}\right)$ and reactive compound $\left({ }^{7} \mathrm{Li}\right)$ into the polymer.

The dependency of the swelling behaviour on the linker concentration and on different swelling temperatures was successfully investigated. As a result, the diffusion into the gel was almost independent of the cross-linker concentration yet varied significantly with temperature. Strikingly, diffusion of LiHMDS was always much slower than that of toluene and at temperatures below $0{ }^{\circ} \mathrm{C}$, diffusion of LiHMDS was barely observed. Apart from providing information about the swelling process itself, observation of small slices rather than the entire sample affords significantly reduced line widths along with a better signal shape, which is most beneficial for crowded spectra or overlapping signals.

Furthermore, a clearly distinguishable ${ }^{7} \mathrm{Li}$ quadrupolar triplet could be observed at the diffusion front after only two days of swelling which would significantly reduce the sample preparation time (in average seven days). Implementation of slice-selective excitation into two-dimensional experiments might therefore promote the employment of the SAG method to the determination of residual dipolar couplings (RDCs) with higher precision or at significantly reduced swelling times. Furthermore, slice-selective excitation could be potentially useful for reaction monitoring. Swelling of the gel in the presence of one reactant and subsequent addition of the other on top of the gel would allow observing their reaction with spatial resolution. A setup where both reactants are separated by the polymer stick would also be thinkable and the diffusion of the reactants towards each other becomes 
accessible. In a first step coloured compounds might be advantageous for the development of such a method, because the NMR observations can be verified optically.

Naturally, slice-selective NMR spectroscopy is also highly useful for polymer chemists as it allows a precisely localized scanning of slices in swollen networks. In this work, butyl acrylate gels were introduced as new polymeric material for strain induced alignment in a gel. PBA gels show enhanced low temperature performance and require only short swelling times, thereby enabling a fast extraction of anisotropic parameters. Although not compatible with organometallic reagents, they may be utile in the analysis of highly flexible or thermally unstable molecules. The nature and swelling properties of PBA were further modulated by utilization of a chain transfer agent during polymerization, which is supposed to control the polymerization process thus yielding a more uniform network. Herein, this aspect was addressed by monitoring the swelling of PBA and PBA / RAFT sticks in the presence of acetone- $d_{8}$ and toluene- $d_{8}$ by slice-selective ${ }^{2} \mathrm{H}$ NMR experiments. The influence of the linker concentration (increasing alignment with increasing crosslinker concentration) was in agreement with the findings for polystyrene. Employment of a RAFT agent led to a significantly smaller alignment yet at an overall higher homogeneity of the gel. This higher quality could facilitate the extraction of anisotropic NMR parameters and increase signal separation. Further, transfer of this procedure to polystyrene was intended and first experiments supported a constant stability towards reactive compounds. In the future work, a thorough investigation of PS/RAFT polymeric material and comparison with pure PS sticks will be necessary to assess its potential to function as improved high-quality alignment medium. The illustrative way in which slice-selective excitation can be used for the characterization of polymer networks could also be beneficial for the analysis of polymeric material obtained by comparable controlling agents in, e.g. ATRP or NMP. The ability to differentiate between the networks from different control mechanisms could allow a better tuning or even choice of a certain polymerization method.
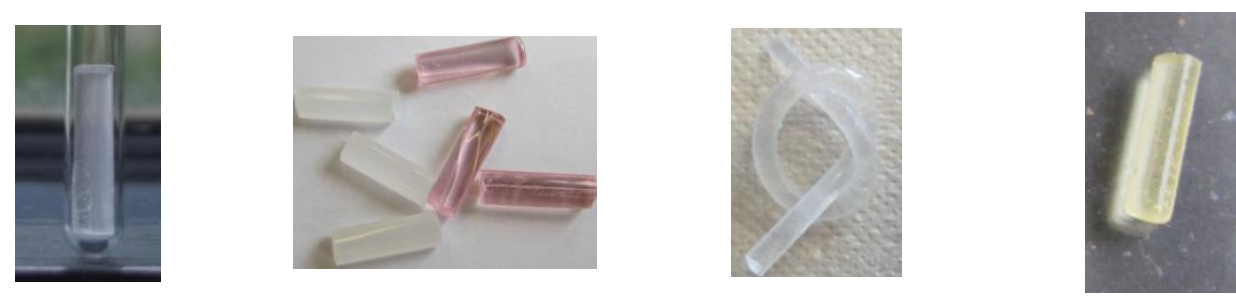

Figure 3.4: PS, PS / RAFT, PBA and PBA / RAFT sticks (from left to right).

In the last part of this thesis, the techniques employed in the preceding chapters were combined with other important analytical tools in chemistry in order to gain detailed insight into the behaviour of more complex systems in solution. In order to understand the structure and reactivity of (Turbo-) Grignard compounds, NMR spectroscopy and ESI 
mass spectrometry were used. While ESI mass spectrometry experiments of $i \mathrm{PrMgCl}$ and $i \mathrm{PrMgCl} \cdot \mathrm{LiCl}$ yielded a very clear picture, this was not the case for NMR spectroscopic experiments in THF-solution. The presence of an equilibrium for both investigated compounds was supported by a low-temperature splitting of the signals belonging to the organic residues, however an exact identification of the involved species was not yet possible. The Turbo-analogue $i \mathrm{PrMgCl} \cdot \mathrm{LiCl}$ displayed the same chemical shfits albeit at increased intensity for the minor peak which is in good accordance with the ESI experiments. By acquiring ${ }^{1} \mathrm{H}$ - and ${ }^{7} \mathrm{Li}$-DOSY spectra it was possible to extract average diffusion coefficients for the two Grignard compounds as well as a reference sample of $\mathrm{LiCl}$. The higher molar mass estimated for the Turbo compound already indicates a more complex dynamic behaviour which is indeed influenced by LiCl. Despite the variety of experiments employed for the characterization of these $i$ Pr-Grignard compounds in solution no final answer can be uncovered and a more detailed investigation of the involved species at low temperature, e.g. by titration of the standard-Grignard compound with $\mathrm{LiCl}$ will become necessary to yield a broader understanding of these highly interesting and widely applied compounds.

Finally, an unprecedented lithium lithiate, [Li(diglyme $\left.)_{2}\right]\left[(\right.$ diglyme $\left.) \mathrm{Li}_{2}\left(\mathrm{C}_{4} \mathrm{H}_{3} \mathrm{~S}\right)_{3}\right]$, was crystallized and analysed in our workgroup. The structure of this compound was investigated upon dissolving in toluene- $d_{8}$ by isotropic and anisotropic NMR spectroscopy as well as DFT calculations. Evaluation of a comprehensive set of NMR experiments uncovered a complex behaviour of this compound in solution. Despite the different environments of the diglyme oxygen atoms, at room temperature only one symmetric set of signals was detectable. This supports fast dissociation-association of the diglyme oxygen atoms from the lithium atoms. The ${ }^{13} \mathrm{C}$ chemical shift of the thienyl- $\mathrm{C}_{a}$ atom, previously utilized as sensitive indicator for the aggregation state of 2-thienyllithium, is in the range of typical dimeric structures. ${ }^{7} \mathrm{Li}$ NMR experiments at $-80{ }^{\circ} \mathrm{C}$ afforded a splitting into at least three different signals, illustrating the presence of further aggregates. Possible aggregates were addressed by DFT calculations at the B3LYP-D3/def2-TZVP level of theory including modelling of solvent effects with the help of the COSMO programme package. Anisotropic NMR experiments supported low symmetry aggregates of which a dimeric arrangement was calculated to be predominantly present in toluene solution. More polar molecules like ether promote the formation of the charged solvent-separated ion pair. This supports on the one hand the single crystal structure analysis, for which crystals were grown from $\mathrm{Et}_{2} \mathrm{O}$, and on the other hand confirms the coexistence of the dimeric aggregate as well as the SSIP in solution due to the presence of small amounts of $\mathrm{Et}_{2} \mathrm{O}$ (from the mother liquor). This underlines again the high benefit gained by combination of different methods, because 
each method by itself was not able to fully explain the existing aggregation phenomena. This last part also very much illustrates how important it is to develop new approaches and to improve existing ones in order to gain a deeper insight into the nature and behaviour of widely employed yet not fully understood compounds.

In summary, it could be repeatedly demonstrated in the course of this work that a combination of crystal structure analysis and NMR spectroscopy is very fruitful. Making SAG accessible for the highly reactive compounds in solution now also permits the determination of anisotropic NMR parameters for organometallic compounds. While the potential of ( $\left.{ }^{7} \mathrm{Li}\right)$ RQCs is not fully exploited, RDCs and RCSAs still need to be investigated. Furthermore, this work illustrates that the applied methods are also advantageous to adjacent fields of research, e.g. polymer science, and that collaborating and broadening the horizon to other techniques yields a more complete understanding. 


\section{EXPERIMENTAL PART}

\subsection{Techniques and Experiments}

\subsubsection{Handling of Air- and Moisture-Sensitive Compounds}

All experimental manipulations were performed either in an inert gas atmosphere of purified dry argon with standard Schlenk techniques ${ }^{[1,219]}$ or in an argon glove box. Glassware was dried at $130^{\circ} \mathrm{C}$, assembled hot and cooled down under vacuum. All solvents were dried over sodium-potassium alloy, distilled and degassed prior to use. Chemicals were either purchased commercially or synthesized according to known literature procedures and stored under inert atmosphere. Solutions of $n$-butyllithium, $i \mathrm{PrMgCl}$ and iPrMgCl-LiCl were kindly donated by ROCKWOOD LITHIUM. $n$-Butyllithium was filtered through Celite ${ }^{\odot}$ before use along with determination of the concentration. ${ }^{[220]}$

\subsubsection{NMR Techniques and Experiments}

All NMR spectra were recorded on a Bruker Avance III $400 \mathrm{MHz}$ spectrometer (Bruker Biospin, Rheinstetten) with a BBFO (broadband-observe) probe, z-gradient and temperature unit. The spectra were measured at $\mathrm{rt}$ if not indicated otherwise. All spectra were processed with Topspin 2.1 (Bruker Biospin, Rheinstetten) and further plotted with MestreNova, Version 7.0 (Mestrelab Research, Santiago de Compostela, Spain). Chemical shifts $(\delta)$ are given in ppm relative to TMS either using the residual solvent signals or tabulated frequency ratios as internal standards. Coupling constants $(J)$ are reported in $\mathrm{Hz}$ and standard abbreviations indicating multiplicity are used as follows: $s=$ singlet, $\mathrm{d}=$ doublet, $\mathrm{t}=$ triplet, sept $=$ septet, $\mathrm{m}=$ multiplet, $\mathrm{br}=$ broad. Axial protons carry the index "a" while equatorial ones are indicated by an "e". All measurements in anisotropic environment as well as all 2D experiments were performed without sample spinning.

Standard ${ }^{1} \mathrm{H}$ NMR spetra (zg30) were measured with 16 scans, a flip angle of $30^{\circ}$ and an acquisition time of $5.1 \mathrm{~s}$. Suppression of polymer signals was afforded by ${ }^{1} \mathrm{H}-\mathrm{CPMG}$ (CarrPurcell-Meiboom-Gill) spectra (cpmg1d) with 1000 cycles of $180^{\circ}$ pulses and a delay of $160 \mu$ s between the $180^{\circ}$ pulses. The quality of the employed polymer gels was monitored by recording ${ }^{2} \mathrm{H}$ NMR spectra ( $z g 2 h$, lock channel) with 16 scans, a flip angle of $30^{\circ}$ and an acquisition time of $5.6 \mathrm{~s}$. 
${ }^{13} \mathrm{C}$-HSQC spectra (hsqcgpph) were measured with $1024 \times 512$ datapoints, 16 or 32 scans and a delay of $1.5 \mathrm{~s}$. Coupled spectra were recorded with the same parameters except for eliminating the decoupling during the acquisition time (PL $12=120 \mathrm{~dB})$.

Exchange spectroscopy (EXSY) measurements were carried out with the standard $90^{\circ}-t_{1}$ $90^{\circ}-\tau_{\mathrm{M}^{-}}-90^{\circ}$ NOESY pulse sequence (noesygpph), $1024 \times 512$ datapoints and 16 dummy scans as well as 16 scans. The mixing time $\mathrm{d} 8$ was set to 0.5 and $0.05 \mathrm{~s}$, respectively, in case of ${ }^{1} \mathrm{H}$ and varied from between 0.005 and $2 \mathrm{~s}$ in case of ${ }^{7} \mathrm{Li}$.

Heteronuclear Overhauser Enhancement Spectroscopy (HOESY) data was obtained by recording spectra with the pulse program hoesyph or hoesygpph $(1024 \times 256$ datapoints). After 16 dummy scans, data was acquired with 16 scans and a mixing time $\mathrm{d} 8$ of $0.5 \mathrm{~s}$. The build-up curves were obtained by varying the mixing time from 0.03 to $1.2 \mathrm{~s}$.

Diffusion experiments (DOSY) were performed with the pulse programme ledbpgp2s employing a linear gradient ramp, 16 dummy scans and 8 scans. The relevant delays for ${ }^{1} \mathrm{H}$-DOSY experiments were $\Delta=100 \mathrm{~ms}$ ("big Delta") and $\delta=2.5 \mathrm{~ms}$ ("little Delta"), whereas for ${ }^{7} \mathrm{Li}$-DOSY measurements $\delta$ was set to $6.4 \mathrm{~ms}$.

For slice-selective excitation (SSE) employed in chapters 2.2.2 and 2.2.3 the value for the gradient strength $(55.7 \mathrm{G} / \mathrm{cm})$ was used as specified by the manufacturer. Uniform and slice-selective ${ }^{2} \mathrm{H}\left(61.4 \mathrm{MHz}\right.$, lock channel) and ${ }^{7} \mathrm{Li}(155.5 \mathrm{MHz})$ NMR spectra were acquired with acquisition times of 1.4 and $0.7 \mathrm{~s}$, and recovery delays of 10.0 and $0.5 \mathrm{~s}$, respectively. To afford slice-selective excitation, p1 determines the length, spnam 1 the shape and spoffs 1 the frequency of the selective pulse. 


\subsection{General Procedures}

\subsubsection{Polymer Synthesis}

Styrene (Fluka, >99\%), DVB (Aldrich, 80\%), BA/EA and BDDA (Aldrich, >90\%) (Scheme 4.1) were freed from the inhibitor by passing through a column of neutral aluminium oxide (Aldrich, Brockmann activity I). Mixtures of the linker unit (0.05-0.6 vol\%) with the corresponding monomers were prepared in a Schlenk flask and degassed thoroughly applying several freeze-pump-thaw cycles.

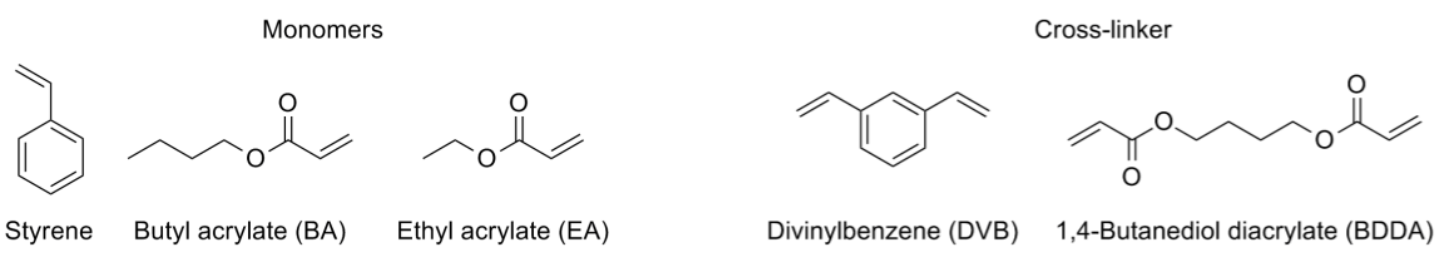

Scheme 4.1: Monomers and corresponding cross-linking units.

Meanwhile, glass tubes (sealed at one end, ID $3.8 \mathrm{~mm}$ ) were treated with a 1:1 mixture of chlorotrimethylsilane and dichlorodimethylsilane for several hours, washed with dichloromethane, and dried in vacuum. The degassed monomer solution was filled into the glass tubes within Schlenk-tubes, which were heated to $115^{\circ} \mathrm{C}$ for polymerization for at least two days.

According to this procedure several distinct polymer sticks were prepared (Table 4.1).

Table 4.1: Summary of the different syntheses and resulting polymer properties.

\begin{tabular}{ccccc}
\hline Monomer & Linker & vol\% linker & T $\left[{ }^{\circ} \mathbf{C}\right]$ & Quality \\
\hline Styrene & DVB & 0.05 & 115 & too soft \\
Styrene & DVB & 0.1 & 115 & good \\
Styrene & DVB & 0.2 & 115 & very good \\
Styrene & DVB & 0.3 & 115 & very good \\
Styrene & DVB & 0.4 & 115 & very good \\
Styrene & DVB & 0.5 & 115 & good \\
Styrene & DVB & 0.6 & 115 & good \\
\hline Butylacrylate & BDDA & 0.6 & 80 & good \\
\hline Butylacrylate & BDDA & 0.3 & 115 & not suitable \\
Butylacrylate & BDDA & 0.6 & 115 & very good \\
\hline Ethylacrylate & BDDA & 0.6 & 115 & good
\end{tabular}


After polymerization, the glass tubes were crashed and remaining glass was removed from the long sticks. Only homogeneous parts of PS and PBA were used and cut into pieces of approximately $1 \mathrm{~cm}$ length. Pure sticks should be nearly colourless and close to transparency. PS sticks are very rigid, whereas PBA and PEA sticks are rather soft and flabby. All sticks were dried in vacuo and then stored in an argon dry box.

\subsubsection{Polymer Swelling}

For polymer swelling, a solution of the compound in question was prepared in an adequate deuterated solvent. Either a) $0.2 \mathrm{~mL}$ of this solution was filled into a $5 \mathrm{~mm}$ NMR tube (ID $4.2 \mathrm{~mm}$ ), and the stick was placed on top of the liquid or $\mathrm{b}$ ) the entire solution was provided in the tube and the polymer stick centred therein. In order to avoid the stick sinking to the bottom of the tube, the NMR tube was held horizontally for 1-2 min until the stick had started to swell. This fixes the stick at this position. In case of preparation a) the missing $0.3 \mathrm{~mL}$ were added on top of the stick.

After approximately one week the swelling was complete for all samples irrespective of the polymer/solvent system and measurements could be performed. The presence of the particular compounds was verified by ${ }^{1} \mathrm{H}-\mathrm{CPMG}$ spectra and the quality of the polymer gels monitored via the quadrupolar splitting in the deuterium NMR spectrum. 


\subsection{Characterizations}

\subsubsection{Synthesis and Characterization of $[t B u L i]_{4} \cdot 4\left[\mathrm{Me}_{2} \mathrm{NC}_{6} \mathrm{H}_{4} \mathrm{Li}\right]_{4}(1)$ :}

To $\mathrm{Me}_{2} \mathrm{NPh}(3.46 \mathrm{~g}, 30.0 \mathrm{mmol}$, 4.0 eq.) $t \mathrm{BuLi}$ in pentane $(1.5 \mathrm{M}, 25.0 \mathrm{~mL}, 37.5 \mathrm{mmol}$, 5.0 eq.) was added at $\mathrm{rt}$. After stirring for $12 \mathrm{~h}$, the yellow solution was reduced in volume and stored at rt. After one day colourless crystals suitable for single crystal X-ray diffraction experiments could be obtained.

Formula:

$\mathrm{C}_{144} \mathrm{H}_{196} \mathrm{Li}_{20} \mathrm{~N}_{16}$

Molecular weight:

$2290.02 \mathrm{~g} / \mathrm{mol}$
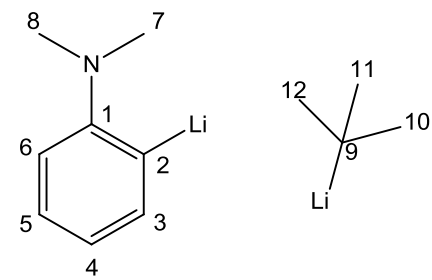

Upon dissolving in toluene- $d_{8}$, five different exchanging species (A-E) were identified unambiguously by applying various 2D NMR techniques:

$\left[\mathrm{Me}_{2} \mathrm{NC}_{6} \mathrm{H}_{4} \mathrm{Li}\right]_{4}(\mathrm{~A})$ :

${ }^{1} \mathrm{H}-\mathrm{NMR}$

(400.13 MHz, tol- $\left.d_{8}\right): \quad \delta=8.21\left(\mathrm{~s}_{\mathrm{br}}, 4 \mathrm{H}, \mathrm{H}_{3}\right), 7.22\left(\mathrm{dd},{ }^{3} J_{\mathrm{HH}}=5.0 \mathrm{~Hz},{ }^{4} J_{\mathrm{HH}}=3.6 \mathrm{~Hz}\right.$, $\left.8 \mathrm{H}, \mathrm{H}_{4,5}\right), 7.00\left(\mathrm{~m}_{\mathrm{c}}, 4 \mathrm{H}, \mathrm{H}_{6}\right), 2.06\left(\mathrm{~s}_{\mathrm{br}}, 24 \mathrm{H}, \mathrm{H}_{7,8}\right) \mathrm{ppm}$.

${ }^{13} \mathrm{C}\left\{{ }^{1} \mathrm{H}\right\}-\mathrm{NMR}$

(100.62 MHz, tol- $\left.\boldsymbol{d}_{8}\right): \quad \delta=168.8\left(\mathrm{~s}_{\mathrm{br}}, 4 \mathrm{C}, \mathrm{C}_{2}\right), 166.2\left(\mathrm{~s}, 4 \mathrm{C}, \mathrm{C}_{1}\right), 140.2\left(\mathrm{~s}, 4 \mathrm{C}, \mathrm{C}_{3}\right)$, 128.0 (s, 4 C, $\left.\mathrm{C}_{4}\right), 126.1$ (s, 4 C, $\mathrm{C}_{5}$ ), 118.9 (s, 4 C, $\mathrm{C}_{6}$ ), 46.9 (s, $\left.8 \mathrm{C}, \mathrm{C}_{7,8}\right) \mathrm{ppm}$.

${ }^{7} \mathrm{Li}-\mathrm{NMR}$

(155.51 MHz, tol- $\left.\boldsymbol{d}_{8}\right): \quad \delta=3.5(\mathrm{~s}) \mathrm{ppm}$.

$\left[\left(\mathrm{Me}_{2} \mathrm{NC}_{6} \mathrm{H}_{4} \mathrm{Li}\right)_{3}(t \mathrm{BuLi})\right](\mathrm{B})$ :

${ }^{1} \mathrm{H}-\mathrm{NMR}$

(400.13 MHz, tol- $\left.d_{8}\right): \quad \delta=7.99\left(\mathrm{~d},{ }^{3} J_{\mathrm{HH}}=5.8 \mathrm{~Hz}, 3 \mathrm{H}, \mathrm{H}_{3}\right), 7.15\left(\mathrm{~m}, 6 \mathrm{H}, \mathrm{H}_{4,5}\right), 6.87(\mathrm{~m}$, $\left.3 \mathrm{H}, \mathrm{H}_{6}\right) 2.14\left(\mathrm{~s}_{\mathrm{br}}, 18 \mathrm{H}, \mathrm{H}_{7,8}\right), 1.21\left(\mathrm{~s}, 9 \mathrm{H}, \mathrm{H}_{10,11,12}\right) \mathrm{ppm}$. 
${ }^{13} \mathrm{C}\left\{{ }^{1} \mathrm{H}\right\}-\mathrm{NMR}$

(100.62 MHz, tol- $\left.\boldsymbol{d}_{8}\right): \quad \delta=168.1\left(\mathrm{sbr}, 3 \mathrm{C}, \mathrm{C}_{2}\right), 165.8\left(\mathrm{~s}, 3 \mathrm{C}, \mathrm{C}_{1}\right), 140.2\left(\mathrm{~s}, 3 \mathrm{C}, \mathrm{C}_{3}\right), 128.0$ $\left(\mathrm{s}, 3 \mathrm{C}, \mathrm{C}_{4}\right), 126.1$ (s, $\left.2 \mathrm{C}, \mathrm{C}_{5}\right), 118.1\left(\mathrm{~s}, 3 \mathrm{C}, \mathrm{C}_{6}\right), 47.2$ (s, $6 \mathrm{C}, \mathrm{C}_{7,8}$ ), 32.3 (s, $\left.3 \mathrm{C}, \mathrm{C}_{10,11,12}\right), 10.6$ (s, $\left.1 \mathrm{C}, \mathrm{C}_{9}\right) \mathrm{ppm}$.

${ }^{7} \mathrm{Li}-\mathrm{NMR}$

(155.51 MHz, tol- $\left.\boldsymbol{d}_{8}\right): \quad \delta=2.7(\mathrm{~s}) \mathrm{ppm}$.

$\left[\left(\mathrm{Me}_{2} \mathrm{NC}_{6} \mathrm{H}_{4} \mathrm{Li}\right)_{2}(t \mathrm{BuLi})_{2}\right](\mathrm{C})$ :

${ }^{1}$ H-NMR

(400.13 MHz, tol- $\left.\boldsymbol{d}_{8}\right): \quad \delta=7.88\left(\mathrm{~m}, 2 \mathrm{H}, \mathrm{H}_{3}\right), 7.14\left(\mathrm{~m}, 4 \mathrm{H}, \mathrm{H}_{4,5}\right), 6.90\left(\mathrm{~m}, 2 \mathrm{H}, \mathrm{H}_{6}\right) 2.02$ $\left(\mathrm{s}_{\mathrm{br}}, 12 \mathrm{H}, \mathrm{H}_{7,8}\right), 1.22\left(\mathrm{~s}_{\mathrm{br}}, 18 \mathrm{H}, \mathrm{H}_{10,11,12}\right) \mathrm{ppm}$.

${ }^{13} \mathrm{C}\left\{{ }^{1} \mathrm{H}\right\}$-NMR

(100.62 MHz, tol- $\left.\boldsymbol{d}_{8}\right): \quad \delta=169.3\left(\mathrm{sbr}_{\mathrm{br}}, 2 \mathrm{C}, \mathrm{C}_{2}\right), 165.5\left(\mathrm{~s}, 2 \mathrm{C}, \mathrm{C}_{1}\right), 140.3\left(\mathrm{~s}, 2 \mathrm{C}, \mathrm{C}_{3}\right), 128.0$ (s, 2 C, $\left.\mathrm{C}_{4}\right), 127.8$ (s, 2 C, $\left.\mathrm{C}_{5}\right), 118.3$ (s, 2 C, $\mathrm{C}_{6}$ ), 46.8 (s, 4 C, $\mathrm{C}_{7,8}$ ), 33.3 (s, $\left.6 \mathrm{C}, \mathrm{C}_{10,11,12}\right), 8.7$ (s, $\left.2 \mathrm{C}, \mathrm{C}_{9}\right) \mathrm{ppm}$.

${ }^{7} \mathrm{Li}-\mathrm{NMR}$

(155.51 MHz, tol- $\left.d_{8}\right): \quad \delta=2.3(\mathrm{~s}) \mathrm{ppm}$.

$\left[\left(\mathrm{Me}_{2} \mathrm{NC}_{6} \mathrm{H}_{4} \mathrm{Li}\right)(t \mathrm{BuLi})_{3}\right](\mathrm{D}):$

${ }^{1} \mathrm{H}-\mathrm{NMR}$

(400.13 MHz, tol- $\left.d_{8}\right): \quad \delta=7.85\left(\mathrm{~m}, 1 \mathrm{H}, \mathrm{H}_{3}\right), 7.12\left(\mathrm{~m}, 2 \mathrm{H}, \mathrm{H}_{4,5}\right), 6.91\left(\mathrm{~m}, 1 \mathrm{H}, \mathrm{H}_{6}\right) 2.11$ $\left(\mathrm{s}_{\mathrm{br}}, 6 \mathrm{H}, \mathrm{H}_{7,8}\right), 1.03\left(\mathrm{~s}_{\mathrm{br}}, 27 \mathrm{H}, \mathrm{H}_{10,11,12}\right) \mathrm{ppm}$.

${ }^{13} \mathrm{C}\left\{{ }^{1} \mathrm{H}\right\}$-NMR

(100.62 MHz, tol- $\left.\boldsymbol{d}_{8}\right): \quad \delta=168.9\left(\mathrm{~s}_{\mathrm{br}}, 2 \mathrm{C}, \mathrm{C}_{2}\right), 166.0\left(\mathrm{~s}, 2 \mathrm{C}, \mathrm{C}_{1}\right), 140.1\left(\mathrm{~s}, 1 \mathrm{C}, \mathrm{C}_{3}\right), 128.1$ (s, 1 C, $\left.\mathrm{C}_{4}\right), 126.1$ (s, $\left.1 \mathrm{C}, \mathrm{C}_{5}\right), 117.9$ (s, $\left.1 \mathrm{C}, \mathrm{C}_{6}\right), 46.9$ (s, $2 \mathrm{C}, \mathrm{C}_{7,8}$ ), 27.3 (s, 9 C, $\left.\mathrm{C}_{10,11,12}\right), 9.5$ (s, 3 C, $\mathrm{C}_{9}$ ) ppm.

\section{${ }^{7}$ Li-NMR}

(155.51 MHz, tol- $\left.\boldsymbol{d}_{8}\right): \quad \delta=1.7$ (s) ppm. 
$[t \mathrm{BuLi}]_{4}(\mathrm{E}):$

${ }^{1} \mathrm{H}-\mathrm{NMR}$

(400.13 MHz, Tol- $\left.\boldsymbol{d}_{8}\right): \quad \delta=0.96\left(\mathrm{~s}, 36 \mathrm{H}, \mathrm{H}_{10,11,12}\right) \mathrm{ppm}$.

${ }^{13} \mathrm{C}\left\{{ }^{1} \mathrm{H}\right\}$-NMR

(100.62 MHz, Tol- $\left.\boldsymbol{d}_{8}\right): \quad \delta=25.9\left(\mathrm{~s}, 12 \mathrm{C}, \mathrm{C}_{10,11,12}\right), 10.9\left(\mathrm{~s}, 4 \mathrm{C}, \mathrm{C}_{9}\right) \mathrm{ppm}$.

\section{${ }^{7} \mathrm{Li}-\mathrm{NMR}$}

(155.51 MHz, Tol- $\left.d_{8}\right): \quad \delta=1.4(\mathrm{~s}) \mathrm{ppm}$.

\subsubsection{Synthesis and Characterization of $\left[\text { ThiLi } \cdot D_{n}\right]_{m}(2 a-e)$}

To a solution of thiophene in diethyl ether a solution of one equivalent of $n \mathrm{BuLi}$ was added over 30 minutes at $0{ }^{\circ} \mathrm{C}$. An excess of the particular donor base (2.5 eq.) was added followed by constant stirring for another $30 \mathrm{~min}$. The solution was then cooled to $-78^{\circ} \mathrm{C}$. The crystals thus formed were filtered, washed twice with pre cooled $n$-hexane $\left(-78^{\circ} \mathrm{C}\right)$ and finally dried in vacuo.

[2-Thienyllithium $\left.\cdot \mathrm{Et}_{2} \mathrm{O}\right]_{4}(2 \mathrm{a})$ :

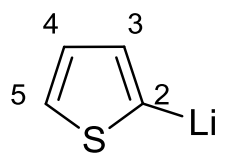

Formula:

Molecular weight:

${ }^{1} \mathrm{H}-\mathrm{NMR}$

(400.13 MHz, tol- $\left.d_{8}\right)$ :

${ }^{13} \mathrm{C}\left\{{ }^{1} \mathrm{H}\right\}$-NMR

(100.62 MHz, tol- $\left.d_{8}\right)$ :

${ }^{7} \mathrm{Li}-\mathrm{NMR}$

(155.51 MHz, tol- $\left.d_{8}\right)$ :
$\mathrm{C}_{32} \mathrm{H}_{52} \mathrm{Li}_{4} \mathrm{O}_{4} \mathrm{~S}_{4}$

$656.74 \mathrm{~g} / \mathrm{mol}$ $\delta=7.80\left(\mathrm{~d},{ }^{3} J_{\mathrm{HH}}=4.3 \mathrm{~Hz}, 1 \mathrm{H}, \mathrm{H}_{5}\right), 7.63\left(\mathrm{~d},{ }^{3} J_{\mathrm{HH}}=2.7 \mathrm{~Hz}, 1 \mathrm{H}\right.$, $\left.\mathrm{H}_{3}\right), 7.33\left(\mathrm{dd},{ }^{3} J_{\mathrm{HH}}=4.3 \mathrm{~Hz},{ }^{3} J_{\mathrm{HH}}=2.8 \mathrm{~Hz}, 1 \mathrm{H}, \mathrm{H}_{4}\right), 3.19(\mathrm{q}$, $\left.{ }^{3} J_{\mathrm{HH}}=7.0 \mathrm{~Hz}, 6 \mathrm{H}, \mathrm{CH}_{3}\right), 0.96\left(\mathrm{t},{ }^{3} J_{\mathrm{HH}}=7.0 \mathrm{~Hz}, 4 \mathrm{H}, \mathrm{CH}_{2}\right)$ ppm.

$\delta=166.9\left(\mathrm{C}_{2}\right), 137.5\left(\mathrm{C}_{5}\right), 133.1\left(\mathrm{C}_{3}\right), 128.4\left(\mathrm{C}_{4}\right), 65.7\left(\mathrm{CH}_{3}\right)$, $15.1\left(\mathrm{CH}_{2}\right) \mathrm{ppm}$.

$\delta=2.1(\mathrm{~s}) \mathrm{ppm}$ 
[2-Thienyllithium $\cdot 2 \mathrm{THF}]_{2}(2 \mathrm{~b})$ :

Formula:

Molecular weight:

${ }^{1} \mathrm{H}-\mathrm{NMR}$

(400.13 MHz, tol- $\left.d_{8}\right)$ :

$\delta=7.88\left(\mathrm{~d},{ }^{3} J_{\mathrm{HH}}=4.3 \mathrm{~Hz}, 1 \mathrm{H}, \mathrm{H}_{5}\right), 7.70\left(\mathrm{dd},{ }^{3} J_{\mathrm{HH}}=2.7 \mathrm{~Hz}\right.$, $\left.{ }^{4} J_{\mathrm{HH}}=2.4 \mathrm{~Hz}, 1 \mathrm{H}, \mathrm{H}_{3}\right), 7.43\left(\mathrm{dd},{ }^{3} J_{\mathrm{HH}}=4.3 \mathrm{~Hz},{ }^{3} J_{\mathrm{HH}}=2.8 \mathrm{~Hz}\right.$, $\left.1 \mathrm{H}, \mathrm{H}_{4}\right), 3.41\left(\mathrm{~m}, 8 \mathrm{H}, \mathrm{OCH}_{2}\right), 1.39\left(\mathrm{~m}, 8 \mathrm{H}, \mathrm{CH}_{2}\right) \mathrm{ppm}$.

${ }^{13} \mathrm{C}\left\{{ }^{1} \mathrm{H}\right\}$-NMR

(100.62 MHz, tol- $\left.d_{8}\right)$ :

$\delta=170.9\left(\mathrm{C}_{2}\right), 137.2\left(\mathrm{C}_{5}\right), 131.6\left(\mathrm{C}_{3}\right), 127.7\left(\mathrm{C}_{4}\right), 65.7\left(\mathrm{OCH}_{2}\right)$, $25.7\left(\mathrm{CH}_{2}\right) \mathrm{ppm}$.

${ }^{7} \mathrm{Li}-\mathrm{NMR}$

(155.51 MHz, tol- $\left.\boldsymbol{d}_{8}\right): \quad \delta=1.9(\mathrm{~s}) \mathrm{ppm}$.
Formula:

Molecular weight:

${ }^{1} \mathrm{H}-\mathrm{NMR}$

(400.13 MHz, tol- $\left.d_{8}\right)$ :

$\delta=7.95\left(\mathrm{dd},{ }^{3} J_{\mathrm{HH}}=4.3 \mathrm{~Hz},{ }^{4} J_{\mathrm{HH}}=0.4 \mathrm{~Hz}, 1 \mathrm{H}, \mathrm{H}_{5}\right), 7.69(\mathrm{dd}$, $\left.{ }^{3} J_{\mathrm{HH}}=2.8 \mathrm{~Hz},{ }^{4} J_{\mathrm{HH}}=0.4 \mathrm{~Hz}, 1 \mathrm{H}, \mathrm{H}_{3}\right), 7.57\left(\mathrm{dd},{ }^{3} J_{\mathrm{HH}}=4.4 \mathrm{~Hz}\right.$, $\left.{ }^{3} \mathrm{~J}_{\mathrm{HH}}=2.8 \mathrm{~Hz}, 1 \mathrm{H}, \mathrm{H}_{4}\right), 3.02\left(\mathrm{~s}, 6 \mathrm{H}, \mathrm{CH}_{3}\right), 2.72\left(\mathrm{~s}, 4 \mathrm{H}, \mathrm{CH}_{2}\right)$ ppm.

${ }^{13} \mathrm{C}\left\{{ }^{1} \mathrm{H}\right\}-\mathrm{NMR}$

(100.62 MHz, tol- $\left.d_{8}\right)$ : $\delta=172.1\left(\mathrm{C}_{2}\right), 136.5\left(\mathrm{C}_{5}\right), 130.7\left(\mathrm{C}_{3}\right), 127.5\left(\mathrm{C}_{4}\right), 70.1\left(\mathrm{CH}_{3}\right)$, $58.8\left(\mathrm{CH}_{2}\right) \mathrm{ppm}$.

\section{${ }^{7}$ Li-NMR}

(155.51 MHz, tol- $\left.\boldsymbol{d}_{\mathbf{8}}\right): \quad \delta=1.7(\mathrm{~s}) \mathrm{ppm}$.
$\mathrm{C}_{16} \mathrm{H}_{26} \mathrm{Li}_{2} \mathrm{O}_{4} \mathrm{~S}_{2}$

$360.39 \mathrm{~g} / \mathrm{mol}$ 
[2-Thienyllithium $\cdot$ TMEDA $]_{2}(2 \mathrm{~d})$ :

Formula:

$\mathrm{C}_{20} \mathrm{H}_{38} \mathrm{Li}_{2} \mathrm{~N}_{4} \mathrm{~S}_{2}$

Molecular weight:

$412.55 \mathrm{~g} / \mathrm{mol}$

${ }^{1} \mathrm{H}-\mathrm{NMR}$

(400.13 MHz, tol- $\left.d_{8}\right): \quad \delta=7.92\left(\mathrm{~d},{ }^{3} J_{\mathrm{HH}}=5.0 \mathrm{~Hz}, 1 \mathrm{H}, \mathrm{H}_{5}\right), 7.66\left(\mathrm{sbr}, 1 \mathrm{H}, \mathrm{H}_{3}\right), 7.50(\mathrm{~d}$, $\left.{ }^{3} J_{\mathrm{HH}}=4.8 \mathrm{~Hz}, 1 \mathrm{H}, \mathrm{H}_{4}\right), 2.28\left(\mathrm{~s}, 12 \mathrm{H}, \mathrm{CH}_{3}\right), 2.09\left(\mathrm{~s}, 4 \mathrm{H}, \mathrm{CH}_{2}\right)$ ppm.

${ }^{13} \mathrm{C}\left\{{ }^{1} \mathrm{H}\right\}$-NMR

(100.62 MHz, tol- $\left.\boldsymbol{d}_{8}\right): \quad \delta=174.4\left(\mathrm{C}_{2}\right), 137.2\left(\mathrm{C}_{5}\right), 130.8\left(\mathrm{C}_{3}\right), 127.4\left(\mathrm{C}_{4}\right), 58.4\left(\mathrm{CH}_{2}\right)$, $46.0\left(\mathrm{CH}_{3}\right) \mathrm{ppm}$.

${ }^{7}$ Li-NMR

(155.51 MHz, tol- $\left.d_{8}\right): \quad \delta=2.0(\mathrm{~s}) \mathrm{ppm}$.

[2-Thienyllithium • PMDETA] (2e):

Formula: $\quad \mathrm{C}_{13} \mathrm{H}_{26} \mathrm{LiN}_{3} \mathrm{~S}$

Molecular weight: $\quad 263.3 \mathrm{~g} / \mathrm{mol}$

${ }^{1} \mathrm{H}-\mathrm{NMR}$

(400.13 MHz, tol- $\left.d_{8}\right): \quad \delta=7.95\left(\mathrm{dd},{ }^{3} J_{\mathrm{HH}}=4.2 \mathrm{~Hz},{ }^{4} J_{\mathrm{HH}}=0.4 \mathrm{~Hz}, 1 \mathrm{H}, \mathrm{H}_{5}\right), 7.64(\mathrm{dd}$, $\left.{ }^{3} J_{\mathrm{HH}}=4.2 \mathrm{~Hz},{ }^{3} J_{\mathrm{HH}}=2.7 \mathrm{~Hz}, 1 \mathrm{H}, \mathrm{H}_{4}\right), 7.49\left(\mathrm{dd},{ }^{3} J_{\mathrm{HH}}=2.7 \mathrm{~Hz}\right.$, $\left.{ }^{4} J_{\mathrm{HH}}=0.4 \mathrm{~Hz}, 1 \mathrm{H}, \mathrm{H}_{3}\right), 2.11\left(\mathrm{~s}, 3 \mathrm{H}, \mathrm{NCH}_{3}\right), 2.01(\mathrm{~s}, 12 \mathrm{H}$, $\left.\mathrm{N}\left(\mathrm{CH}_{3}\right)_{2}\right), 1.86\left(\mathrm{br}, 8 \mathrm{H}, \mathrm{CH}_{2}\right)$ ppm.

${ }^{13} \mathrm{C}\left\{{ }^{1} \mathrm{H}\right\}-\mathrm{NMR}$

(100.62 MHz, tol- $\left.d_{8}\right): \quad \quad \delta=180.1 \quad\left(C_{2}\right), \quad 137.5 \quad\left(C_{5}\right), 133.3 \quad\left(C_{3}\right) \quad 126.8 \quad\left(C_{4}\right), \quad 57.3$ $\left(\mathrm{Me}_{2} \mathrm{NCH}_{2}\right), 53.9\left(\mathrm{CH}_{2} \mathrm{NMe}\right), 45.9\left(\mathrm{~N}\left(\mathrm{CH}_{3}\right)_{2}\right), 44.6\left(\mathrm{NCH}_{3}\right)$ ppm.

\section{${ }^{7} \mathrm{Li}-\mathrm{NMR}$}

(155.51 MHz, tol- $\left.\boldsymbol{d}_{8}\right): \quad \delta=2.1(\mathrm{~s}) \mathrm{ppm}$. 


\subsubsection{Characterization of (-)-Menthol (3)}

(-)-Menthol (50 mg) was dissolved in toluene- $d_{8}(0.5 \mathrm{~mL})$ and put to swell with a polystyrene stick ( 0.6 vol\%) according to the general procedure reported above. After two weeks of swelling the sample was analysed by NMR spectroscopic measurements.

Formula:

Molecular weight:

$$
\mathrm{C}_{10} \mathrm{H}_{20} \mathrm{O}
$$

$156.3 \mathrm{~g} / \mathrm{mol}$

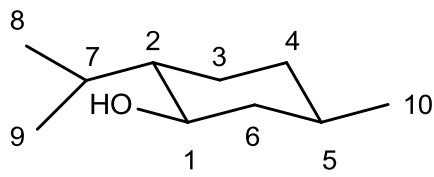

${ }^{1} \mathrm{H}-\mathrm{NMR}$

(400.13 MHz, tol- $\left.d_{8}\right): \quad \delta=3.13\left(\mathrm{~m}, 1 \mathrm{H}, \mathrm{H}_{1}\right), 2.23\left(\mathrm{dsept},{ }^{3} J_{\mathrm{HH}}=7.0,2.9 \mathrm{~Hz}, 1 \mathrm{H}, \mathrm{H}_{7}\right)$, $1.71\left(\mathrm{~m}, 1 \mathrm{H}, \mathrm{H}_{6 \mathrm{e}}\right), 1.67(\mathrm{~s}, 1 \mathrm{H}, \mathrm{OH}), 1.51\left(\mathrm{~m}, 1 \mathrm{H}, \mathrm{H}_{4 \mathrm{e}}\right), 1.46(\mathrm{~m}$, $\left.1 \mathrm{H}, \mathrm{H}_{3 \mathrm{e}}\right), 1.17\left(\mathrm{~m}, 1 \mathrm{H}, \mathrm{H}_{5}\right), 0.99\left(\mathrm{~m}, 1 \mathrm{H}, \mathrm{H}_{2}\right), 0.92(\mathrm{~d}$, $\left.{ }^{3} J_{\mathrm{HH}}=7.4 \mathrm{~Hz}, 3 \mathrm{H}, \mathrm{H}_{8}\right), 0.85\left(\mathrm{~d},{ }^{3} J_{\mathrm{HH}}=6.2 \mathrm{~Hz}, 3 \mathrm{H}, \mathrm{H}_{10}\right), 0.83(\mathrm{~m}$, $\left.1 \mathrm{H}, \mathrm{H}_{6 \mathrm{a}}\right), 0.82\left(\mathrm{~d},{ }^{3} \mathrm{~J}_{\mathrm{HH}}=7.4 \mathrm{~Hz}, 3 \mathrm{H}, \mathrm{H}_{9}\right), 0.77\left(\mathrm{~m}, 1 \mathrm{H}, \mathrm{H}_{3 \mathrm{a}}\right), 0.7$ $\left(\mathrm{m}, 1 \mathrm{H}, \mathrm{H}_{4 \mathrm{a}}\right)$ ppm.

${ }^{13} \mathrm{C}-\mathrm{NMR}$

$\left(100.62 \mathrm{MHz}\right.$, tol- $\left.d_{8}\right)$ : $\delta=71.3\left(\mathrm{~s}, 1 \mathrm{C}, \mathrm{C}_{1}\right), 50.5\left(\mathrm{~s}, 1 \mathrm{C}, \mathrm{C}_{2}\right), 45.8\left(\mathrm{~s}, 1 \mathrm{C}, \mathrm{C}_{6}\right), 35.1(\mathrm{~s}, 1 \mathrm{C}$, $\mathrm{C}_{4}$ ), 32.0 (s, 1C, $\mathrm{C}_{5}$ ), 26.3 (s, 1C, $\mathrm{C}_{7}$ ), 23.8 (s, 1C, $\mathrm{C}_{3}$ ), 22.5 (s, 1C, $\left.\mathrm{C}_{10}\right), 21.3\left(\mathrm{~s}, 1 \mathrm{C}, \mathrm{C}_{8}\right), 16.7\left(\mathrm{~s}, 1 \mathrm{C}, \mathrm{C}_{9}\right) \mathrm{ppm}$.

\begin{tabular}{c|c}
\hline Atom & $\boldsymbol{D}(\mathbf{C}-\mathrm{H})$ in $\mathbf{H z}$ \\
\hline $\mathrm{C}_{1}$ & -23.0 \\
\hline $\mathrm{C}_{2}$ & -18.8 \\
\hline $\mathrm{C}_{3}-\mathrm{H}_{\mathrm{e}}$ & -4.3 \\
\hline $\mathrm{C}_{3}-\mathrm{H}_{\mathrm{a}}$ & -32.7 \\
\hline $\mathrm{C}_{4}-\mathrm{H}_{\mathrm{e}}$ & -4.3 \\
\hline $\mathrm{C}_{4}-\mathrm{H}_{\mathrm{a}}$ & -30.1 \\
\hline $\mathrm{C}_{5}$ & -24.1 \\
\hline $\mathrm{C}_{6}-\mathrm{H}_{\mathrm{e}}$ & -7.6 \\
\hline $\mathrm{C}_{6}-\mathrm{H}_{a}$ & -19.6 \\
\hline $\mathrm{C}_{7}$ & -14.5 \\
\hline $\mathrm{C}_{8}$ & 1.1 \\
\hline $\mathrm{C}_{9}$ & 3.2 \\
\hline $\mathrm{C}_{10}$ & -8.9 \\
\hline
\end{tabular}




\subsubsection{Characterization of Lithium hexamethyldisilazane (4)}

Lithium hexamethyldisilazane $(50 \mathrm{mg}, 0.30 \mathrm{mmol})$ was dissolved in toluene- $d_{8}(0.5 \mathrm{~mL})$. $0.2 \mathrm{~mL}$ of the resulting solution were transferred into an NMR sample tube. After adjusting a polystyrene stick on top, the remaining $0.3 \mathrm{~mL}$ were added. An isotropic reference sample was prepared with the same quantities. For the slice-selective experiments, samples with 0.2, 0.3 and 0.4 vol\% cross-linker were prepared and put to swell at room temperature. Additional samples with 0.3 vol\% were put to swell at $+40,+4$ and $-20{ }^{\circ} \mathrm{C}$, while the measurements itself were all carried out at room temperature.

Formula:

Molecular weight:

${ }^{1} \mathrm{H}-\mathrm{NMR}$

(400.13 MHz, Tol- $\left.\boldsymbol{d}_{8}\right): \quad \delta=0.40(\mathrm{~s}, 18 \mathrm{H}) \mathrm{ppm}$.

${ }^{13} \mathrm{C}\left\{{ }^{1} \mathrm{H}\right\}-\mathrm{NMR}$

(100.62 MHz, Tol- $\left.\boldsymbol{d}_{8}\right): \quad \delta=5.6\left(\mathrm{~s}, 6 \mathrm{C}, \mathrm{CH}_{3}\right) \mathrm{ppm}$.

\section{${ }^{7} \mathrm{Li}-\mathrm{NMR}$}

(155.52 MHz, Tol- $\left.\boldsymbol{d}_{\mathbf{8}}\right): \quad \delta=1.1(\mathrm{~s}) \mathrm{ppm}$.

${ }^{29} \mathrm{Si}-\mathrm{NMR}$

(79.50 MHz, Tol- $\left.\boldsymbol{d}_{\mathbf{8}}\right): \quad \delta=-11.4(\mathrm{~s}) \mathrm{ppm}$. 


\subsubsection{Characterization of $n$ Butyllithium $\cdot L(5 a-d)$}

A defined amount of $n \mathrm{BuLi}$ in hexane was dried in vacuo, transferred into an argon-dry box and dissolved in toluene- $d_{8}$. Anisotropic samples were prepared according to the general procedure either directly to obtain the unsolvated hexameric aggregate (a) or after subsequent addition of the corresponding Lewis base $\mathrm{L}\left(\mathrm{Et}_{2} \mathrm{O}(\mathrm{b})\right.$, THF (c), PMDETA (d)). Again, isotropic reference samples were prepared with the same quantities. After one week the swollen samples were ready for NMR spectroscopic measurements. In the following only the ${ }^{7} \mathrm{Li}$ chemical shifts are depicted due to the direct relevance for this work.

\section{${ }^{7} \mathbf{L i}-\mathbf{N M R}$}

$$
\begin{aligned}
& \text { (155.52 MHz, tol- } \left.d_{8}\right): \quad \delta=2.3 \mathrm{ppm} . \quad n \text {-butyllithium } \\
& \delta=2.1 \mathrm{ppm} . \quad n \text {-butyllithium } \cdot \mathrm{Et}_{2} \mathrm{O} \\
& \delta=1.6 \mathrm{ppm} . \quad n \text {-butyllithium } \cdot \mathrm{THF} \\
& \delta=1.1 \mathrm{ppm} . \quad n \text {-butyllithium.PMDETA }
\end{aligned}
$$

\subsubsection{Characterization of Lithiumtetrafluoroborate (6)}

Lithiumtetrafluoroborate was dissolved in THF- $d_{8}$. The mixture was further applied to swell a PS stick ( 0.3 vol\% DVB) for 6 days and quadrupolar splittings were obtained for the ${ }^{7} \mathrm{Li}$ and ${ }^{11} \mathrm{~B}$ nuclei of $\mathrm{LiBF}_{4}$.

Formula:

Molecular weight:

\section{${ }^{7}$ Li-NMR}

(155.51 MHz, PS / THF- $\left.\boldsymbol{d}_{\mathbf{8}}\right): \delta=0.8\left(\mathrm{t}, \Delta v_{\mathrm{Q}}=5.5 \mathrm{~Hz}\right) \mathrm{ppm}$.

\section{${ }^{11} \mathrm{~B}-\mathrm{NMR}$}

(128.38 MHz, PS / THF- $\left.\boldsymbol{d}_{8}\right): \delta=0.2\left(\mathrm{t}, \Delta v_{\mathrm{Q}}=126 \mathrm{~Hz}\right) \mathrm{ppm}$.

\section{${ }^{19}$ F-NMR}

(128.38 MHz, PS / THF- $\boldsymbol{d}_{\mathbf{8}}$ ): $\delta=156.9$ (s) ppm. 


\subsubsection{Characterization of $i \mathrm{PrMgCl}(7)$}

Isotropic samples were prepared by adding THF- $d_{8}(0.4 \mathrm{~mL})$ to a solution of $i \mathrm{PrMgCl}$ in THF $(1.35 \mathrm{M}, 0.3 \mathrm{~mL})$. The anisotropic sample was prepared by primarily swelling the PS stick in pure THF- $d_{8}$ followed by the addition of $i \mathrm{PrMgCl} \cdot \mathrm{LiCl}$ to the already swollen gel. For DOSY experiments low concentrated samples of $i \mathrm{PrMgCl}$ in $\mathrm{THF}(1.35 \mathrm{M}, 22 \mu \mathrm{L})$ with THF- $d_{8}(0.5 \mathrm{~mL})$ were prepared.

\section{${ }^{1} \mathrm{H}-\mathrm{NMR}$}

(400.13 MHz, THF- $\left.\boldsymbol{d}_{8}\right): \quad \delta=1.12\left(\mathrm{~d},{ }^{3} J_{\mathrm{HH}}=7.8 \mathrm{~Hz}, 1 \mathrm{H}, \mathrm{CH}\right),-0.53\left(\mathrm{sept},{ }^{3} J_{\mathrm{HH}}=7.8 \mathrm{~Hz}\right.$, $\left.6 \mathrm{H}, \mathrm{CH}_{3}\right) \mathrm{ppm}$.

${ }^{13} \mathrm{C}\left\{{ }^{1} \mathrm{H}\right\}-\mathrm{NMR}$

(100.62 MHz, THF- $\left.\boldsymbol{d}_{\mathbf{8}}\right): \quad \delta=25.6\left(\mathrm{~s}, 2 \mathrm{C}, \mathrm{CH}_{3}\right), 9.3(\mathrm{~s}, 1 \mathrm{C}, \mathrm{CH}) \mathrm{ppm}$.

\subsubsection{Characterization of $\mathrm{iPrMgCl} \cdot \mathrm{LiCl}(8)$}

Isotropic samples were prepared by adding THF- $d_{8}(0.4 \mathrm{~mL})$ to a solution of $i \mathrm{PrMgCl} \cdot \mathrm{LiCl}$ in THF $(0.95 \mathrm{M}, 0.3 \mathrm{~mL})$. The anisotropic sample was prepared by primarily swelling the PS stick in pure THF- $d_{8}$ followed by the addition of $i \mathrm{PrMgCl} \cdot \mathrm{LiCl}$ to the already swollen gel. For DOSY experiments low concentrated samples of $i \mathrm{PrMgCl} \cdot \mathrm{LiCl}$ in $\mathrm{THF}(0.95 \mathrm{M}, 20 \mu \mathrm{L})$ with THF- $d_{8}(0.5 \mathrm{~mL})$ were prepared.

\section{${ }^{1} \mathrm{H}-\mathrm{NMR}$}

(400.13 MHz, THF- $\left.\boldsymbol{d}_{\mathbf{8}}\right): \quad \delta=1.09\left(\mathrm{~d},{ }^{3} J_{\mathrm{HH}}=7.8 \mathrm{~Hz}, 1 \mathrm{H}, \mathrm{CH}\right),-0.55\left(\mathrm{sept},{ }^{3} J_{\mathrm{HH}}=7.8 \mathrm{~Hz}\right.$, $\left.6 \mathrm{H}, \mathrm{CH}_{3}\right) \mathrm{ppm}$.

${ }^{13} \mathrm{C}\left\{{ }^{1} \mathrm{H}\right\}$-NMR

(100.62 MHz, THF- $\left.\boldsymbol{d}_{8}\right): \quad \delta=25.6\left(\mathrm{~s}, 2 \mathrm{C}, \mathrm{CH}_{3}\right), 9.8(\mathrm{~s}, 1 \mathrm{C}, \mathrm{CH}) \mathrm{ppm}$.

\section{${ }^{7}$ Li-NMR}

(155.51 MHz, THF- $\left.\boldsymbol{d}_{8}\right): \quad \delta=0.0(\mathrm{~s}) \mathrm{ppm}$. 


\subsubsection{Characterization of $\mathrm{LiCl}(9)$}

To THF- $d_{8}(360 \mu \mathrm{L})$ a solution of dry $\mathrm{LiCl}$ in THF $(0.5 \mathrm{M}, 140 \mu \mathrm{L})$ was added.

\section{${ }^{7} \mathbf{L i}-\mathbf{N M R}$}

(155.51 MHz, THF- $\left.d_{8}\right): \quad \delta=0.4(\mathrm{~s}) \mathrm{ppm}$.

\subsubsection{Characterization of [Li(diglyme $\left.)_{2}\right]\left[(\right.$ diglyme $\left.) L_{2}\left(\mathrm{C}_{4} \mathrm{H}_{3} \mathrm{~S}\right)_{3}\right](10)$}

Crystals of (10) were taken from the mother liquor and directly dissolved in toluene- $d_{8}$. Thereof, an isotropic and an anisotropic sample (PS / tol- $d_{8}, 0.2$ vol\% DVB) were prepared. Thereby, a set of RDCs could be obtained by measuring F2-coupled HSQC spectra.

Formula:

Molecular weight:

${ }^{1} \mathrm{H}-\mathrm{NMR}$

(400.13 MHz, tol- $\left.d_{8}\right)$ :

$\delta=7.89\left(\mathrm{~d},{ }^{3} J_{\mathrm{HH}}=4.1 \mathrm{~Hz}, 3 \mathrm{H}, \mathrm{H}_{5}\right), 7.65\left(\mathrm{~d},{ }^{3} J_{\mathrm{HH}}=2.8 \mathrm{~Hz}, 3 \mathrm{H}\right.$, $\left.\mathrm{H}_{3}\right), 7.51\left(\mathrm{dd},{ }^{3} J_{\mathrm{HH}}=4.1,2.8 \mathrm{~Hz}, 3 \mathrm{H}, \mathrm{H}_{4}\right), 3.21\left(\mathrm{~m}, 12 \mathrm{H}, \mathrm{H}_{\mathrm{1}^{\prime}}\right)$, $3.16\left(\mathrm{~m}, 12 \mathrm{H}, \mathrm{H}_{2^{\prime}}\right), 3.09\left(\mathrm{~d}, 18 \mathrm{H}, \mathrm{CH}_{3}\right) \mathrm{ppm}$.

${ }^{13} \mathrm{C}\left\{{ }^{1} \mathrm{H}\right\}-\mathrm{NMR}$

(100.61 MHz, tol- $\left.\boldsymbol{d}_{8}\right): \quad \delta=172.5\left(\mathrm{~s}, 3 \mathrm{C}, \mathrm{C}_{2}\right), 136.3\left(\mathrm{~s}, 3 \mathrm{C}, \mathrm{C}_{3}\right), 130.0\left(\mathrm{~s}, 3 \mathrm{C}, \mathrm{C}_{5}\right), 127.1$ $\left.\left(\mathrm{s}, 3 \mathrm{C}, \mathrm{C}_{4}\right), 71.2\left(\mathrm{~s}, 6 \mathrm{C}, \mathrm{C}_{2}\right)^{2}\right), 69.6\left(\mathrm{~s}, 6 \mathrm{C}, \mathrm{C}_{1^{\prime}}\right), 58.6\left(\mathrm{~s}, 6 \mathrm{C}, \mathrm{C}_{3}\right)$ ppm.

\section{${ }^{7} \mathrm{Li}-\mathrm{NMR}$}

(155.51 MHz, Tol- $\left.d_{8}\right): \quad \delta=1.6(\mathrm{~s}) \mathrm{ppm}$.

$$
\mathrm{C}_{30} \mathrm{H}_{51} \mathrm{Li}_{3} \mathrm{O}_{9} \mathrm{~S}_{3}
$$

$672.7 \mathrm{~g} / \mathrm{mol}$

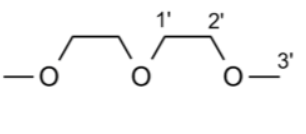

\section{$(155.51 \mathrm{MH}, \mathrm{T}$ - $\mathrm{ds}): \mathrm{s}=1.6(\mathrm{~s}) \mathrm{ppm}$}

RDCs for (10)

\begin{tabular}{c|c}
\hline Atom & $\boldsymbol{D}(\mathbf{C}-\mathrm{H})$ in $\mathbf{H z}$ \\
\hline $\mathrm{C}_{3}$ & 15.9 \\
\hline $\mathrm{C}_{4}$ & 0.1 \\
\hline $\mathrm{C}_{5}$ & 1.0 \\
\hline $\mathrm{C}_{1^{\prime}}$ & 0.8 \\
\hline $\mathrm{C}_{2^{\prime}}$ & 6.3 \\
\hline $\mathrm{C}_{3^{\prime}}$ & 2.1 \\
\hline $\mathrm{H}_{4^{-}}-\mathrm{H}_{5}$ & 4.0 \\
\hline
\end{tabular}




\section{Appendix}

\section{BP86-D3/def2-SVP level of theory}

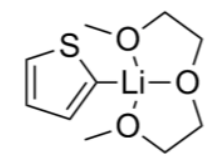

32

Coordinates from ORCA-job opt_lids_a_bp86d_svp

\begin{tabular}{|c|c|c|c|}
\hline $\mathrm{C}$ & -5.189186 & 0.401700 & 0.057015 \\
\hline $\mathrm{O}$ & -3.827752 & 0.860816 & -0.024701 \\
\hline $\mathrm{C}$ & -3.670618 & 2.261631 & 0.198842 \\
\hline $\mathrm{C}$ & -3.581793 & 3.005964 & -1.134928 \\
\hline $\mathrm{O}$ & -2.602171 & 2.416684 & -1.999277 \\
\hline $\mathrm{C}$ & -1.222931 & 2.642582 & -1.707122 \\
\hline $\mathrm{C}$ & -0.596192 & 1.391398 & -1.068512 \\
\hline $\mathrm{O}$ & -1.002876 & 0.202641 & -1.749923 \\
\hline $\mathrm{Li}$ & -3.002364 & 0.331178 & -1.894104 \\
\hline $\mathrm{C}$ & -4.526481 & -0.361731 & -3.027616 \\
\hline $\mathrm{C}$ & -5.303690 & -1.524957 & -3.085445 \\
\hline $\mathrm{C}$ & -6.656144 & -1.383368 & -3.558738 \\
\hline $\mathrm{C}$ & -6.977366 & -0.077228 & -3.881902 \\
\hline $\mathrm{S}$ & -5.575979 & 0.934193 & -3.609583 \\
\hline $\mathrm{C}$ & -0.314673 & -0.065607 & -2.975305 \\
\hline $\mathrm{H}$ & -5.565666 & 0.511799 & 1.099574 \\
\hline $\mathrm{H}$ & -5.192433 & -0.660273 & -0.246696 \\
\hline $\mathrm{H}$ & -5.848815 & 0.943595 & -0.655256 \\
\hline $\mathrm{H}$ & -2.737338 & 2.402668 & 0.787889 \\
\hline $\mathrm{H}$ & -4.504945 & 2.675638 & 0.810896 \\
\hline $\mathrm{H}$ & -3.370034 & 4.089257 & -0.971190 \\
\hline $\mathrm{H}$ & -4.537508 & 2.910615 & -1.691566 \\
\hline $\mathrm{H}$ & -0.727216 & 2.875576 & -2.674481 \\
\hline $\mathrm{H}$ & -1.074833 & 3.523640 & -1.041078 \\
\hline $\mathrm{H}$ & 0.515915 & 1.482589 & -1.046387 \\
\hline $\mathrm{H}$ & -0.948732 & 1.264317 & -0.024914 \\
\hline $\mathrm{H}$ & -4.904066 & -2.507228 & -2.775580 \\
\hline $\mathrm{H}$ & -7.367009 & -2.218869 & -3.664459 \\
\hline $\mathrm{H}$ & -7.921016 & 0.311092 & -4.289566 \\
\hline $\mathrm{H}$ & -0.744630 & -0.998165 & -3.385159 \\
\hline $\mathrm{H}$ & 0.774392 & -0.208085 & -2.791133 \\
\hline $\mathrm{H}$ & -0.458403 & 0.743559 & -3.726554 \\
\hline
\end{tabular}

monomer 
64

Coordinates from ORCA-job opt_li2d2s2_a_bp86d_svp

\begin{tabular}{|c|c|c|c|}
\hline $\mathrm{C}$ & -4.830652 & 2.951937 & -1.695081 \\
\hline $\mathrm{O}$ & -4.591781 & 1.555423 & -1.746459 \\
\hline $\mathrm{C}$ & -3.224673 & 1.245119 & -1.567533 \\
\hline $\mathrm{C}$ & -3.046639 & -0.263392 & -1.670066 \\
\hline $\mathrm{O}$ & -1.653340 & -0.527953 & -1.509788 \\
\hline $\mathrm{C}$ & -1.314281 & -1.904603 & -1.358983 \\
\hline $\mathrm{C}$ & 0.198612 & -1.949879 & -1.173854 \\
\hline $\mathrm{O}$ & 0.870248 & -1.340136 & -2.278320 \\
\hline $\mathrm{Li}$ & -0.241725 & 0.342730 & -2.75721 \\
\hline $\mathrm{C}$ & -0.810406 & -0.043146 & -4.811970 \\
\hline $\mathrm{Li}$ & -1.136806 & 2.066024 & -4.324676 \\
\hline $\mathrm{O}$ & -0.541088 & 3.244231 & -5.812967 \\
\hline $\mathrm{C}$ & -1.556978 & 3.458621 & -6.79231 \\
\hline $\mathrm{C}$ & -2.581708 & 2.349440 & -6.64346 \\
\hline $\mathrm{O}$ & -3.015021 & 2.323053 & -5.281521 \\
\hline $\mathrm{C}$ & -4.144869 & 1.470554 & -5.08956 \\
\hline $\mathrm{C}$ & 0.642955 & 4.032204 & -5.94946 \\
\hline $\mathrm{C}$ & 0.374277 & 5.534221 & -6.13756 \\
\hline $\mathrm{O}$ & -0.589409 & 6.072148 & -5.262766 \\
\hline $\mathrm{C}$ & -0.216657 & 6.036508 & -3.890525 \\
\hline $\mathrm{C}$ & 0.052199 & -0.078200 & -5.917838 \\
\hline $\mathrm{C}$ & -0.156449 & -1.136102 & -6.871580 \\
\hline $\mathrm{C}$ & -1.205188 & -1.967509 & -6.521918 \\
\hline$S$ & -1.905361 & -1.402687 & -5.02394 \\
\hline $\mathrm{C}$ & 0.078649 & 2.458269 & -2.53961 \\
\hline $\mathrm{C}$ & 1.436372 & 2.791440 & -2.69115 \\
\hline $\mathrm{C}$ & 1.956532 & 3.800378 & -1.80905 \\
\hline $\mathrm{C}$ & 0.996655 & 4.278204 & -0.93321 \\
\hline$S$ & -0.514585 & 3.459254 & -1.220449 \\
\hline $\mathrm{C}$ & 1.204656 & -2.242759 & -3.338959 \\
\hline $\mathrm{H}$ & -5.918566 & 3.110290 & -1.827277 \\
\hline $\mathrm{H}$ & -4.282738 & 3.494790 & -2.503592 \\
\hline $\mathrm{H}$ & -4.515498 & 3.390198 & -0.717626 \\
\hline $\mathrm{H}$ & -2.844315 & 1.601329 & -0.579441 \\
\hline $\mathrm{H}$ & -2.586796 & 1.742892 & -2.340803 \\
\hline $\mathrm{H}$ & -3.410395 & -0.636888 & -2.655343 \\
\hline $\mathrm{H}$ & -3.634365 & -0.771100 & -0.869806 \\
\hline $\mathrm{H}$ & -1.628851 & -2.482253 & -2.261143 \\
\hline $\mathrm{H}$ & -1.823253 & -2.344159 & -0.466980 \\
\hline $\mathrm{H}$ & 0.472376 & -1.361985 & -0.272136 \\
\hline $\mathrm{H}$ & 0.540274 & -2.999016 & -1.021702 \\
\hline $\mathrm{H}$ & -1.134042 & 3.425161 & -7.824856 \\
\hline $\mathrm{H}$ & -2.032024 & 4.451688 & -6.626937 \\
\hline $\mathrm{H}$ & -2.139712 & 1.361561 & -6.917855 \\
\hline $\mathrm{H}$ & -3.441039 & 2.557713 & -7.324795 \\
\hline
\end{tabular}

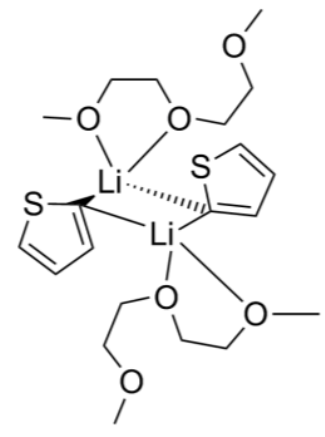

dimer 


$\begin{array}{rrrr}\mathrm{H} & -3.918914 & 0.428607 & -5.410888 \\ \mathrm{H} & -5.021900 & 1.850572 & -5.663549 \\ \mathrm{H} & -4.395087 & 1.465593 & -4.012465 \\ \mathrm{H} & 1.254558 & 3.666722 & -6.811301 \\ \mathrm{H} & 1.209873 & 3.842781 & -5.017297 \\ \mathrm{H} & -0.003140 & 5.736054 & -7.162700 \\ \mathrm{H} & 1.362471 & 6.058125 & -6.050497 \\ \mathrm{H} & -0.997989 & 6.580807 & -3.326124 \\ \mathrm{H} & 0.765188 & 6.540766 & -3.715785 \\ \mathrm{H} & -0.152637 & 5.002997 & -3.486209 \\ \mathrm{H} & 0.849763 & 0.673601 & -6.053695 \\ \mathrm{H} & 0.445638 & -1.279664 & -7.782636 \\ \mathrm{H} & -1.585872 & -2.846941 & -7.059011 \\ \mathrm{H} & 2.076782 & 2.294609 & -3.442361 \\ \mathrm{H} & 2.997731 & 4.159035 & -1.817711 \\ \mathrm{H} & 1.112389 & 5.045406 & -0.154730 \\ \mathrm{H} & 1.640636 & -1.636763 & -4.153427 \\ \mathrm{H} & 0.306593 & -2.754325 & -3.750770 \\ \mathrm{H} & 1.944464 & -2.997963 & -2.987041\end{array}$

49

Coordinates from ORCA-job opt_li2ds3m_a_bp86d_svp

$\begin{array}{lrrr}\mathrm{Li} & 3.984708 & 2.723047 & 11.861983 \\ \mathrm{Li} & 1.462728 & 1.588810 & 11.529075 \\ \mathrm{C} & 4.835723 & 4.073171 & 9.327370 \\ \mathrm{H} & 5.344909 & 3.996013 & 8.336060 \\ \mathrm{H} & 5.448984 & 4.701137 & 10.002401 \\ \mathrm{H} & 3.838356 & 4.551889 & 9.194629 \\ \mathrm{O} & 4.717202 & 2.794834 & 9.939034 \\ \mathrm{C} & 3.919891 & 1.877877 & 9.186333 \\ \mathrm{H} & 4.334400 & 1.746105 & 8.157051 \\ \mathrm{H} & 2.871769 & 2.241827 & 9.093166 \\ \mathrm{C} & 3.937007 & 0.539993 & 9.898466 \\ \mathrm{H} & 4.974327 & 0.126744 & 9.929203 \\ \mathrm{H} & 3.264208 & -0.168761 & 9.363327 \\ \mathrm{O} & 3.475839 & 0.745897 & 11.237882 \\ \mathrm{C} & 3.418790 & -0.450587 & 12.019285 \\ \mathrm{H} & 4.430762 & -0.915736 & 12.088326 \\ \mathrm{H} & 2.717921 & -1.166911 & 11.528930 \\ \mathrm{C} & 2.958710 & -0.084983 & 13.418867 \\ \mathrm{H} & 2.804492 & -1.021100 & 14.009946 \\ \mathrm{H} & 3.742749 & 0.528709 & 13.916357 \\ \mathrm{O} & 1.743504 & 0.658642 & 13.314743 \\ \mathrm{C} & 1.391114 & 1.326891 & 14.521811 \\ \mathrm{H} & 0.539401 & 1.995665 & 14.292263 \\ \mathrm{H} & 2.241581 & 1.937929 & 14.900113\end{array}$

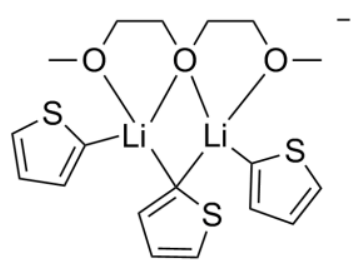




$\begin{array}{lrrr}\mathrm{H} & 1.090022 & 0.595769 & 15.309974 \\ \mathrm{C} & 5.272935 & 2.430705 & 13.471451 \\ \mathrm{~S} & 4.923586 & 2.963228 & 15.110734 \\ \mathrm{C} & 6.224841 & 1.408380 & 13.605736 \\ \mathrm{H} & 6.630455 & 0.878022 & 12.724520 \\ \mathrm{C} & 6.636687 & 1.069255 & 14.943796 \\ \mathrm{H} & 7.380599 & 0.291659 & 15.188823 \\ \mathrm{C} & 6.002226 & 1.835218 & 15.908042 \\ \mathrm{H} & 6.143241 & 1.804948 & 16.998616 \\ \mathrm{C} & 0.595226 & 0.638348 & 9.867536 \\ \mathrm{~S} & 0.576568 & -1.111547 & 9.659028 \\ \mathrm{C} & 0.476104 & 1.164452 & 8.572326 \\ \mathrm{H} & 0.451915 & 2.256159 & 8.398146 \\ \mathrm{C} & 0.391788 & 0.222910 & 7.485424 \\ \mathrm{H} & 0.287639 & 0.508382 & 6.424694 \\ \mathrm{C} & 0.440079 & -1.094728 & 7.910499 \\ \mathrm{H} & 0.366808 & -2.008007 & 7.301995 \\ \mathrm{C} & 1.986709 & 3.685729 & 11.760792 \\ \mathrm{~S} & 1.310395 & 4.590914 & 10.407604 \\ \mathrm{C} & 2.105492 & 4.608795 & 12.811993 \\ \mathrm{H} & 2.511272 & 4.315739 & 13.796370 \\ \mathrm{C} & 1.686818 & 5.958310 & 12.540478 \\ \mathrm{H} & 1.726977 & 6.779113 & 13.275575 \\ \mathrm{C} & 1.219533 & 6.120327 & 11.247254 \\ \mathrm{H} & 0.823240 & 7.032790 & 10.778916\end{array}$

47

Coordinates from ORCA-job opt_lid2p_bp86d_svp

$\begin{array}{lrrr}\mathrm{Li} & 7.769363 & 4.267548 & 3.953489 \\ \mathrm{O} & 9.004696 & 5.713900 & 2.974540 \\ \mathrm{O} & 6.206156 & 4.129041 & 2.494938 \\ \mathrm{C} & 9.517726 & 5.511545 & 1.658998 \\ \mathrm{C} & 5.475982 & 5.277845 & 2.071909 \\ \mathrm{O} & 7.098280 & 6.090437 & 4.766807 \\ \mathrm{O} & 8.391341 & 2.672312 & 2.746562 \\ \mathrm{C} & 8.583568 & 7.055663 & 3.213669 \\ \mathrm{C} & 6.521926 & 3.236169 & 1.428509 \\ \mathrm{O} & 6.467202 & 3.611192 & 5.519721 \\ \mathrm{O} & 9.446583 & 3.492327 & 5.049608 \\ \mathrm{C} & 8.064617 & 7.130361 & 4.638674 \\ \mathrm{C} & 7.319366 & 2.083941 & 2.013213 \\ \mathrm{C} & 6.516469 & 5.918010 & 6.057944 \\ \mathrm{C} & 9.260582 & 1.771251 & 3.431720 \\ \mathrm{C} & 5.642004 & 4.681634 & 5.968869 \\ \mathrm{C} & 10.226526 & 2.634055 & 4.222675 \\ \mathrm{C} & 5.771130 & 2.375573 & 5.398472\end{array}$




$\begin{array}{rrrr}\mathrm{C} & 10.231560 & 4.369478 & 5.849353 \\ \mathrm{H} & 9.538264 & 4.909714 & 6.521767 \\ \mathrm{H} & 10.960045 & 3.806708 & 6.475426 \\ \mathrm{H} & 10.781972 & 5.105570 & 5.219309 \\ \mathrm{H} & 10.899269 & 1.986133 & 4.832486 \\ \mathrm{H} & 10.862908 & 3.234850 & 3.528471 \\ \mathrm{H} & 8.673825 & 1.115324 & 4.118481 \\ \mathrm{H} & 9.816008 & 1.120837 & 2.717223 \\ \mathrm{H} & 6.683541 & 1.466354 & 2.689833 \\ \mathrm{H} & 7.696366 & 1.425556 & 1.196765 \\ \mathrm{H} & 5.598892 & 2.848038 & 0.937714 \\ \mathrm{H} & 7.125179 & 3.765093 & 0.651922 \\ \mathrm{H} & 4.466197 & 5.000249 & 1.695415 \\ \mathrm{H} & 6.017175 & 5.825942 & 1.265701 \\ \mathrm{H} & 5.381077 & 5.939518 & 2.952338 \\ \mathrm{H} & 5.004856 & 2.421577 & 4.589819 \\ \mathrm{H} & 5.275674 & 2.091651 & 6.354197 \\ \mathrm{H} & 6.520660 & 1.600804 & 5.150350 \\ \mathrm{H} & 4.801273 & 4.853607 & 5.253544 \\ \mathrm{H} & 5.200335 & 4.458913 & 6.968568 \\ \mathrm{H} & 7.776802 & 7.337370 & 2.495258 \\ \mathrm{H} & 9.426240 & 7.773973 & 3.080152 \\ \mathrm{H} & 10.459380 & 6.082254 & 1.498723 \\ \mathrm{H} & 5.907124 & 6.805114 & 6.347506 \\ \mathrm{H} & 7.312679 & 5.775327 & 6.826542 \\ \mathrm{H} & 7.613847 & 8.131950 & 4.829789 \\ \mathrm{H} & 8.894247 & 6.980100 & 5.368425 \\ \mathrm{H} & 8.777870 & 5.820122 & 0.884019 \\ \mathrm{H} & 9.711046 & 4.428269 & 1.555086\end{array}$

Reaction energies:

\begin{tabular}{c|c|c|c} 
reaction & $\begin{array}{c}\text { gas phase } \\
\text { energy }[\mathrm{kJ} / \mathrm{mol}]\end{array}$ & $\begin{array}{c}\text { COSMO (tol) } \\
\text { energy }[\mathrm{kJ} / \mathrm{mol}]\end{array}$ & $\begin{array}{c}\text { combination } \\
\text { energy }[\mathrm{kJ} / \mathrm{mol}]\end{array}$ \\
\hline 1 & -112.36 & -69.69 & -76.16 \\
$2 \mathrm{a}$ & 465.71 & 173.63 & 180.57 \\
$2 \mathrm{~b}$ & 64.31 & -17.72 & -23.96 \\
$2 \mathrm{c}$ & 176.67 & 51.97 & 52.21
\end{tabular}




\section{B3LYP-D3/def2-TZVP level of theory}

32

Coordinates from ORCA-job lids_b3d_tzvp<smiles>CO[Te]1(c2cccs2)CCCO1</smiles>

$\begin{array}{lrrr}\mathrm{C} & -5.488651 & 1.134906 & 0.127071 \\ \mathrm{O} & -4.053544 & 1.130905 & 0.167808 \\ \mathrm{C} & -3.482695 & 2.386534 & 0.538483 \\ \mathrm{C} & -3.272906 & 3.242117 & -0.698602 \\ \mathrm{O} & -2.587091 & 2.494805 & -1.704502 \\ \mathrm{C} & -1.160460 & 2.534641 & -1.716622 \\ \mathrm{C} & -0.592253 & 1.220179 & -1.185144 \\ \mathrm{O} & -1.304331 & 0.098811 & -1.702815 \\ \mathrm{Li} & -3.275087 & 0.467864 & -1.558032 \\ \mathrm{C} & -4.621376 & -0.404990 & -2.812106 \\ \mathrm{C} & -5.013324 & -1.705090 & -3.084846 \\ \mathrm{C} & -6.092631 & -1.871358 & -4.010083 \\ \mathrm{C} & -6.572061 & -0.682000 & -4.481140 \\ \mathrm{~S} & -5.667250 & 0.610721 & -3.765621 \\ \mathrm{C} & -1.046019 & -0.200471 & -3.083798 \\ \mathrm{H} & -5.887311 & 1.428990 & 1.102988 \\ \mathrm{H} & -5.802626 & 0.126418 & -0.125930 \\ \mathrm{H} & -5.865010 & 1.811691 & -0.643658 \\ \mathrm{H} & -2.529870 & 2.162825 & 1.021507 \\ \mathrm{H} & -4.117132 & 2.907704 & 1.263544 \\ \mathrm{H} & -2.733764 & 4.160828 & -0.446722 \\ \mathrm{H} & -4.232353 & 3.519164 & -1.138562 \\ \mathrm{H} & -0.861444 & 2.697106 & -2.753675 \\ \mathrm{H} & -0.780944 & 3.370684 & -1.122561 \\ \mathrm{H} & 0.474802 & 1.147573 & -1.425408 \\ \mathrm{H} & -0.706775 & 1.159594 & -0.101583 \\ \mathrm{H} & -4.532009 & -2.560447 & -2.618401 \\ \mathrm{H} & -6.497749 & -2.830918 & -4.310572 \\ \mathrm{H} & -7.376758 & -0.516672 & -5.182307 \\ \mathrm{H} & -1.663534 & -1.059349 & -3.333800 \\ \mathrm{H} & 0.013101 & -0.434157 & -3.227430 \\ \mathrm{H} & -1.333493 & 0.629013 & -3.734435 \\ & & & \\ & & & \end{array}$

64

Coordinates from ORCA-job opt_li2d2s2_b3d_tzvp

$\begin{array}{llll}\mathrm{C} & -4.442547 & 3.425154 & -2.109693 \\ \mathrm{O} & -4.365895 & 2.023500 & -1.900708 \\ \mathrm{C} & -3.031291 & 1.592018 & -1.697253 \\ \mathrm{C} & -3.023366 & 0.076948 & -1.622967\end{array}$




\begin{tabular}{|c|c|c|c|}
\hline $\mathrm{O}$ & -1.669638 & -0.335501 & -1.434364 \\
\hline $\mathrm{C}$ & -1.522427 & -1.694373 & -1.024387 \\
\hline $\mathrm{C}$ & -0.040159 & -1.923175 & -0.813037 \\
\hline $\mathrm{O}$ & 0.686911 & -1.673704 & -2.018250 \\
\hline $\mathrm{Li}$ & -0.134138 & 0.029482 & -2.843887 \\
\hline $\mathrm{C}$ & -0.558016 & -0.359374 & -4.900975 \\
\hline $\mathrm{Li}$ & -0.831676 & 1.793608 & -4.465756 \\
\hline $\mathrm{O}$ & -0.506356 & 3.226245 & -5.822042 \\
\hline $\mathrm{C}$ & -1.510717 & 3.324402 & -6.825504 \\
\hline $\mathrm{C}$ & -2.375691 & 2.091856 & -6.713367 \\
\hline $\mathrm{O}$ & -2.804433 & 2.000196 & -5.352121 \\
\hline $\mathrm{C}$ & -4.092366 & 1.416341 & -5.192339 \\
\hline $\mathrm{C}$ & 0.404376 & 4.328177 & -5.731253 \\
\hline $\mathrm{C}$ & -0.235735 & 5.714789 & -5.796790 \\
\hline $\mathrm{O}$ & -1.320144 & 5.920237 & -4.915344 \\
\hline $\mathrm{C}$ & -0.968816 & 5.949942 & -3.538358 \\
\hline C & 0.370535 & -0.221971 & -5.925241 \\
\hline $\mathrm{C}$ & 0.118435 & -0.952244 & -7.125204 \\
\hline $\mathrm{C}$ & -1.032314 & -1.686369 & -7.055560 \\
\hline$S$ & -1.761832 & -1.454060 & -5.505765 \\
\hline $\mathrm{C}$ & 0.493610 & 2.108692 & -2.728189 \\
\hline $\mathrm{C}$ & 1.782834 & 2.477832 & -3.084751 \\
\hline $\mathrm{C}$ & 2.418634 & 3.465578 & -2.272552 \\
\hline $\mathrm{C}$ & 1.626286 & 3.876117 & -1.236361 \\
\hline $\mathrm{S}$ & 0.123711 & 3.030449 & -1.299791 \\
\hline $\mathrm{C}$ & 0.809601 & -2.810856 & -2.876064 \\
\hline $\mathrm{H}$ & -5.488527 & 3.678778 & -2.279453 \\
\hline $\mathrm{H}$ & -3.855200 & 3.725834 & -2.986498 \\
\hline $\mathrm{H}$ & -4.067678 & 3.979636 & -1.239974 \\
\hline $\mathrm{H}$ & -2.620252 & 2.015405 & -0.773445 \\
\hline $\mathrm{H}$ & -2.394212 & 1.933443 & -2.520397 \\
\hline $\mathrm{H}$ & -3.430605 & -0.355110 & -2.544312 \\
\hline $\mathrm{H}$ & -3.643208 & -0.254864 & -0.783177 \\
\hline $\mathrm{H}$ & -1.926715 & -2.365036 & -1.792559 \\
\hline $\mathrm{H}$ & -2.068855 & -1.873445 & -0.090450 \\
\hline $\mathrm{H}$ & 0.333547 & -1.211576 & -0.074434 \\
\hline $\mathrm{H}$ & 0.147507 & -2.937518 & -0.447159 \\
\hline $\mathrm{H}$ & -1.059688 & 3.385460 & -7.823316 \\
\hline $\mathrm{H}$ & -2.123984 & 4.210304 & -6.643170 \\
\hline $\mathrm{H}$ & -1.827079 & 1.185101 & -6.988806 \\
\hline $\mathrm{H}$ & -3.235036 & 2.201745 & -7.381870 \\
\hline $\mathrm{H}$ & -4.101660 & 0.384663 & -5.556733 \\
\hline $\mathrm{H}$ & -4.841418 & 2.002038 & -5.736398 \\
\hline $\mathrm{H}$ & -4.330598 & 1.429658 & -4.131951 \\
\hline $\mathrm{H}$ & 1.142320 & 4.262219 & -6.541511 \\
\hline $\mathrm{H}$ & 0.926359 & 4.181186 & -4.788610 \\
\hline $\mathrm{H}$ & -0.631984 & 5.915891 & -6.793872 \\
\hline $\mathrm{H}$ & 0.567471 & 6.443553 & -5.610432 \\
\hline
\end{tabular}

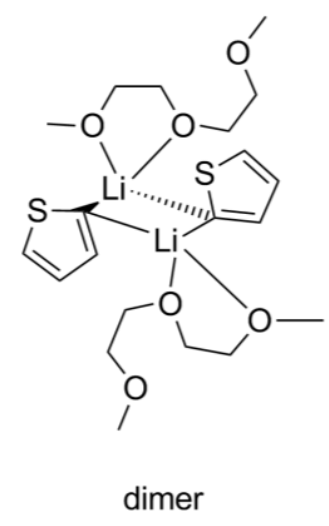




$\begin{array}{lrrr}\mathrm{H} & -1.872707 & 6.213782 & -2.989333 \\ \mathrm{H} & -0.201192 & 6.708526 & -3.340815 \\ \mathrm{H} & -0.608807 & 4.981508 & -3.183759 \\ \mathrm{H} & 1.242942 & 0.418693 & -5.832572 \\ \mathrm{H} & 0.763433 & -0.932167 & -7.995426 \\ \mathrm{H} & -1.456721 & -2.334001 & -7.807729 \\ \mathrm{H} & 2.283237 & 2.046801 & -3.946237 \\ \mathrm{H} & 3.415263 & 3.850332 & -2.452219 \\ \mathrm{H} & 1.854701 & 4.600079 & -0.468088 \\ \mathrm{H} & 1.342224 & -2.477133 & -3.762572 \\ \mathrm{H} & -0.168042 & -3.189085 & -3.185096 \\ \mathrm{H} & 1.372929 & -3.601001 & -2.370078\end{array}$

49

Coordinates from ORCA-job opt_li2ds3m_b3d_tzvp

$\begin{array}{lrrr}\mathrm{Li} & 4.078327 & 2.512920 & 11.809779 \\ \mathrm{Li} & 1.589178 & 1.440169 & 11.362715 \\ \mathrm{C} & 4.907162 & 3.606440 & 9.188709 \\ \mathrm{H} & 5.560744 & 3.487330 & 8.317333 \\ \mathrm{H} & 5.321643 & 4.362768 & 9.852962 \\ \mathrm{H} & 3.913517 & 3.924677 & 8.860308 \\ \mathrm{O} & 4.837095 & 2.391547 & 9.929992 \\ \mathrm{C} & 4.219337 & 1.326508 & 9.205846 \\ \mathrm{H} & 4.784050 & 1.110400 & 8.290330 \\ \mathrm{H} & 3.192232 & 1.594954 & 8.935787 \\ \mathrm{C} & 4.216060 & 0.099336 & 10.083358 \\ \mathrm{H} & 5.238558 & -0.214937 & 10.322020 \\ \mathrm{H} & 3.692900 & -0.708016 & 9.561395 \\ \mathrm{O} & 3.536737 & 0.423684 & 11.297711 \\ \mathrm{C} & 3.439712 & -0.652867 & 12.227256 \\ \mathrm{H} & 4.430834 & -1.078748 & 12.419858 \\ \mathrm{H} & 2.789061 & -1.431012 & 11.810102 \\ \mathrm{C} & 2.880975 & -0.114261 & 13.518942 \\ \mathrm{H} & 2.800461 & -0.932311 & 14.246430 \\ \mathrm{H} & 3.550060 & 0.655053 & 13.917897 \\ \mathrm{O} & 1.594505 & 0.441431 & 13.251960 \\ \mathrm{C} & 1.008281 & 1.064697 & 14.390004 \\ \mathrm{H} & 0.093845 & 1.551901 & 14.056783 \\ \mathrm{H} & 1.677802 & 1.818189 & 14.811872 \\ \mathrm{H} & 0.771058 & 0.316823 & 15.155067 \\ \mathrm{C} & 5.418666 & 2.486617 & 13.402713 \\ \mathrm{~S} & 5.580916 & 3.797659 & 14.531975 \\ \mathrm{C} & 6.255000 & 1.484737 & 13.873595 \\ \mathrm{H} & 6.354116 & 0.530933 & 13.360240 \\ \mathrm{C} & 6.976123 & 1.755528 & 15.079373 \\ \mathrm{H} & 7.651673 & 1.052944 & 15.555752 \\ & & & \\ & & & \end{array}$<smiles>COCC[Te](c1cccs1)(c1cccs1)[Te](OC)(c1cccs1)c1cccs1</smiles> 


$\begin{array}{lrrr}\mathrm{C} & 6.719668 & 3.006051 & 15.572623 \\ \mathrm{H} & 7.129247 & 3.469731 & 16.458001 \\ \mathrm{C} & 0.690921 & 0.832683 & 9.558426 \\ \mathrm{~S} & 0.857825 & -0.720225 & 8.780037 \\ \mathrm{C} & 0.146633 & 1.669248 & 8.595539 \\ \mathrm{H} & -0.059211 & 2.714070 & 8.803299 \\ \mathrm{C} & -0.113936 & 1.097778 & 7.310514 \\ \mathrm{H} & -0.537413 & 1.646970 & 6.476559 \\ \mathrm{C} & 0.226629 & -0.225079 & 7.241962 \\ \mathrm{H} & 0.123513 & -0.896946 & 6.402099 \\ \mathrm{C} & 2.116773 & 3.531797 & 11.805456 \\ \mathrm{~S} & 1.496033 & 4.573628 & 10.549994 \\ \mathrm{C} & 1.962951 & 4.243404 & 12.986775 \\ \mathrm{H} & 2.291875 & 3.846413 & 13.940487 \\ \mathrm{C} & 1.378343 & 5.541967 & 12.887473 \\ \mathrm{H} & 1.200565 & 6.195703 & 13.733441 \\ \mathrm{C} & 1.062642 & 5.874694 & 11.598368 \\ \mathrm{H} & 0.606543 & 6.784989 & 11.237822\end{array}$

47

Coordinates from ORCA-job opt_lid2p_b3d_tzvp

$\begin{array}{lrrr}\mathrm{Li} & 7.834024 & 4.281581 & 3.912459 \\ \mathrm{O} & 9.131369 & 5.855842 & 3.061794 \\ \mathrm{O} & 6.247326 & 4.153344 & 2.474627 \\ \mathrm{C} & 9.673441 & 5.753388 & 1.746511 \\ \mathrm{C} & 5.560873 & 5.304177 & 1.986322 \\ \mathrm{O} & 7.094976 & 6.045226 & 4.735997 \\ \mathrm{O} & 8.355379 & 2.607567 & 2.685706 \\ \mathrm{C} & 8.629861 & 7.161121 & 3.344447 \\ \mathrm{C} & 6.440505 & 3.163453 & 1.467103 \\ \mathrm{O} & 6.435998 & 3.593053 & 5.604478 \\ \mathrm{O} & 9.551808 & 3.464586 & 4.928288 \\ \mathrm{C} & 8.009568 & 7.136958 & 4.720973 \\ \mathrm{C} & 7.195508 & 2.023708 & 2.101110 \\ \mathrm{C} & 6.319266 & 5.926852 & 5.926467 \\ \mathrm{C} & 9.195255 & 1.704438 & 3.402247 \\ \mathrm{C} & 5.514967 & 4.657486 & 5.803490 \\ \mathrm{C} & 10.239918 & 2.540891 & 4.094472 \\ \mathrm{C} & 5.827612 & 2.308843 & 5.663798 \\ \mathrm{C} & 10.419530 & 4.319515 & 5.664038 \\ \mathrm{H} & 9.792965 & 4.933732 & 6.308808 \\ \mathrm{H} & 11.103631 & 3.733746 & 6.286181 \\ \mathrm{H} & 10.991829 & 4.964921 & 4.989924 \\ \mathrm{H} & 10.898070 & 1.897640 & 4.689806 \\ \mathrm{H} & 10.848897 & 3.081212 & 3.358768\end{array}$




$\begin{array}{lrrr}\mathrm{H} & 8.603867 & 1.150420 & 4.141315 \\ \mathrm{H} & 9.666239 & 0.988007 & 2.720686 \\ \mathrm{H} & 6.589674 & 1.536522 & 2.874152 \\ \mathrm{H} & 7.466737 & 1.277777 & 1.346903 \\ \mathrm{H} & 5.479021 & 2.812912 & 1.075405 \\ \mathrm{H} & 7.022110 & 3.586586 & 0.639253 \\ \mathrm{H} & 4.571301 & 5.031971 & 1.606478 \\ \mathrm{H} & 6.130539 & 5.784599 & 1.183350 \\ \mathrm{H} & 5.462338 & 5.994260 & 2.819574 \\ \mathrm{H} & 5.119003 & 2.168923 & 4.839441 \\ \mathrm{H} & 5.299530 & 2.169172 & 6.612661 \\ \mathrm{H} & 6.625171 & 1.571777 & 5.591485 \\ \mathrm{H} & 4.821299 & 4.717746 & 4.955350 \\ \mathrm{H} & 4.931567 & 4.502815 & 6.718773 \\ \mathrm{H} & 7.872419 & 7.432953 & 2.600303 \\ \mathrm{H} & 9.436230 & 7.902395 & 3.306054 \\ \mathrm{H} & 10.542313 & 6.407896 & 1.630705 \\ \mathrm{H} & 5.653001 & 6.789244 & 6.038493 \\ \mathrm{H} & 6.980524 & 5.876334 & 6.799217 \\ \mathrm{H} & 7.489157 & 8.081316 & 4.914873 \\ \mathrm{H} & 8.770542 & 6.996881 & 5.496804 \\ \mathrm{H} & 8.921702 & 6.022034 & 0.995832 \\ \mathrm{H} & 9.970971 & 4.717378 & 1.599575\end{array}$

Reaction energies:

\begin{tabular}{c|c|c|c|c|c|c} 
reaction & $\begin{array}{c}\text { gas phase } \\
\text { B3LYP-D3 } \\
{[\mathrm{kJ} / \mathrm{mol}]}\end{array}$ & $\begin{array}{c}\text { gas phase } \\
\text { LCCSD(T0) } \\
{[\mathrm{kJ} / \mathrm{mol}]}\end{array}$ & $\begin{array}{c}\varepsilon=2.4 \\
\text { B3LYP-D3 } \\
{[\mathrm{kJ} / \mathrm{mol}]}\end{array}$ & $\begin{array}{c}\varepsilon=2.4 \\
\text { LCCSD(T0) } \\
{[\mathrm{kJ} / \mathrm{mol}]}\end{array}$ & $\begin{array}{c}\varepsilon=4.3 \\
\text { B3LYP-D3 } \\
{[\mathrm{kJ} / \mathrm{mol}]}\end{array}$ & $\begin{array}{c}\varepsilon=4.3 \\
\text { LCCSD(T0) } \\
{[\mathrm{kJ} / \mathrm{mol}]}\end{array}$ \\
\hline $\mathbf{1}$ & -79.72 & -59.30 & -42.57 & -22.15 & -24.86 & -4.44 \\
$\mathbf{2 a}$ & 464.58 & 450.27 & 178.11 & 163.80 & 53.99 & 39.68 \\
$\mathbf{2 b}$ & 112.71 & 136.19 & 25.20 & 48.67 & -10.30 & 13.18 \\
$\mathbf{2 c}$ & 192.43 & 195.48 & 67.77 & 70.82 & 14.57 & 17.62
\end{tabular}




\section{References}

[1] W. Schlenk, J. Holtz, Ber. Dtsch. Chem. Ges. 1917, 50, 262-274.

[2] a) B. J. Wakefield, in The Chemistry of Organolithium Compounds, Pergamon Press, Oxford, New York, 1974; b) M. Schlosser, in Organometallics in Synthesis: A Manual (Ed.: M. Schlosser), Wiley-VCH, New York, 2002, pp. 1-166; c) J. Clayden, Organolithiums: Selectivity for Synthesis, Vol. 1, Elsevier Science (Pergamon), Oxford, 2002.

[3] a) P. Beak, A. Basu, D. J. Gallagher, Y. S. Park, S. Thayumanavan, Acc. Chem. Res. 1996, 29, 552-560; b) A. Basu, S. Thayumanavan, Angew. Chem. 2002, 114, 740-763; Angew. Chem. Int. Ed. 2002, 41, 716-738.

[4] G. Wu, M. Huang, Chem. Rev. 2006, 106, 2596-2616.

[5] H. Dietrich, Acta Crystallogr., Sect. E. 1963, 16, 681-689.

[6] T. Stey, D. Stalke, in The chemistry of organolithium compounds (Eds.: Z. Rappoport, I. Marek), John Wiley \& Sons, Chichester (GB), 2004, pp. 47-120 and references cited therein.

[7] a) E. Weiss, Angew. Chem. 1993, 105, 1565-1587; Angew. Chem. Int. Ed. Engl. 1993, 32, 1501-1523.; b) V. H. Gessner, C. Däschlein, C. Strohmann, Chemistry - A European Journal 2009, 15, 3320-3334.

[8] a) S. Harder, J. Boersma, L. Brandsma, J. A. Kanters, W. Bauer, P. v. R. Schleyer, Organometallics 1989, 8, 1696-1700; b) R. D. Thomas, R. M. Jensen, T. C. Young, Organometallics 1987, 6, 565-571; c) T. Tatic, S. Hermann, D. Stalke, Organometallics 2012, 31, 5615-5621.

[9] a) G. Wittig, F. J. Meyer, G. Lange, Justus Liebigs Ann. Chem. 1951, 571, 167-201; b) C. Eaborn, P. B. Hitchcock, J. D. Smith, A. C. Sullivan, J. Chem. Soc., Chem. Commun. 1983, 0, 827-828; c) R. Michel, R. Herbst-Irmer, D. Stalke Organometallics 2011, 30, 4379-4386.

[10] M. Granitzka, PhD Thesis, Göttingen (Germany) 2013.

[11] A. V. Yakimansky, A. H. E. Müller, M. Van Beylen, Macromolecules 2000, 33, 5686-5692.

[12] M. G. Davidson, R. Snaith, D. Stalke, D. S. Wright, J. Org. Chem. 1993, 58, 2810-2816.

[13] S. P. Patterman, I. L. Karle, G. D. Stucky, J. Am. Chem. Soc. 1970, 92, 1150-1157.

[14] E. Weiss, E. A. C. Lucken, J. Organomet. Chem. 1964, 2, 197-205

[15] R. L. Gerteis, R. E. Dickerson, T. L. Brown, Inorg. Chem. 1964, 3, 872-875.

[16] A. Wheatly, Chem. Soc. Rev. 2001, 30, 265-273.

[17] P. West, P. Waack, J. Am. Chem. Soc. 1966, 89, 4395-4399.

[18] M. Veith, Bärnighausen, Acta Cryst. 1974, B30, 1806-1813. 
[19] a) D. Seebach, R. Amstutz, T. Laube, W. B. Schweizer, J. D. Dunitz, J. Am. Chem. Soc. 1985, 107, 54035409; b) H. Hope, Acta Crystallogr. 1988, B44, 22-26; c) R. Boese, D. Bläser, J. Appl. Cryst. 1989, 22, 394395.

[20] T. Kottke, D. Stalke, J. Appl. Crystallogr. 1993, 26, 615-619.

[21] R. Michel, private communication 2012.

[22] D. Stalke, Chem. Soc. Rev. 1998, 27, 171-178.

[23] T. Kottke, D. Stalke, Angew. Chem. 1993, 105, 619-621; Angew. Chem. Int. Ed. Engl. 1993, 32, 580-582.

[24] U. S. Siemeling, T. Redecker, B. Neumann, H.-G. Stammler, J. Am. Chem. Soc. 1994, 116, 5507-5508.

[25] R. Zerger, W. Rhine, G. Stucky, J. Am. Chem. Soc. 1974, 96, 6048-6055.

[26] E. Weiss, G. Hencken, J. Organomet. Chem. 1970, 21, 265-268.

[27] H. Dietrich, J. Organomet. Chem. 1981, 205, 291-299.

[28] M. A. Nichols, P. G. Williard, J. Am. Chem. Soc. 1993, 115, 1568-1572.

[29] C. Strohmann, T. Seibel, K. Strohfeldt, Angew. Chem. 2003, 115, 4669-4671; Angew. Chem. Int. Ed. 2003, 42, 4531-4533.

[30] H. Ott, C. Däschlein, D. Leusser, D. Schildbach, T. Seibel, D. Stalke, C. Strohmann, J. Am. Chem. Soc. 2008, 130, 11901-11911.

[31] C. Lambert, P. von Ragué Schleyer, Angew. Chem. 1994, 106, 1187-1199; Angew. Chem. Int. Ed. Engl. 1994, 33, 1129-1140.

[32] a) J. B. Collins, A. J. Streiwieser, J. Comput. Chem. 1980, 1, 81-87; b) J. P. Ritchie, S. M. Bachrach, J. Am. Chem. Soc. 1987, 109, 5909-5916; c) W. Scherer, P. Sirsch, M. Grosche, M. Spiegler, S. A. Mason, M. G. Gardiner, Chem. Comm. 2001, 2072-2073; d) N. Kocher, J. Henn, B. Gostevskii, D. Kost, I. Kalikhman, B. Engels, D. Stalke, J. Am. Chem. Soc. 2004, 136, 5563-5568; e) J. Henn, D. Leusser, D. Stalke, J. Comput. Chem. 2007, 28, 2317-2324.

[33] a) T. L. Brown, in Adv. Organomet. Chem., Vol. Volume 3 (Eds.: F. G. A. Stone, W. Robert), Academic Press, 1966, pp. 365-395; b) H. Günther, D. Moskau, P. Bast, D. Schmalz, Angew. Chem. 1987, 99, $1242-$ 1250; Angew. Chem. Int. Ed. Engl. 1987, 26, 1212-1220; c) H. Günther, J. Braz. Chem. Soc. 1999, 10, 241262; d) G. Fraenkel, in The Chemistry of Organolithium Compounds, John Wiley \& Sons, Ltd, 2006, pp. $1-61$.

[34] T. L. Brown, D. W. Dickerhoof, D. A. Bafus, J. Am. Chem. Soc. 1962, 84, 1371-1376.

[35] a) P. A. Scherr, R. J. Hogan, J. P. Oliver, J. Am. Chem. Soc. 1974, 96, 6055-6059; b) J. A. Ladd, J. Parker, J. Chem. Soc., Dalton Trans. 1972, 0, 930-934.

[36] R. D. Thomas, M. T. Clarke, T. Corby Young, J. Organomet. Chem. 1987, 328, 239-248.

[37] a) L. D. McKeever, R. Waack, M. A. Doran, E. B. Baker, J. Am. Chem. Soc. 1968, 90, 3244-3244; b) L. D. McKeever, R. Waack, M. A. Doran, E. B. Baker, J. Am. Chem. Soc. 1969, 91, 1057-1061. 
[38] R. Knorr, T. Menke, K. Ferchland, J. Mehlstäubl, D. S. Stephenson, J. Am. Chem. Soc. 2008, 130, 1417914188.

[39] a) G. Fraenkel, M. Heinrichs, J. M. Hewitt, B. M. Su, J. M. Geckle, J. Am. Chem. Soc. 1980, 102, 33453350; b) D. Seebach, R. Hässig, J. Gabriel, Helv. Chim. Acta 1983, 66, 308-337; c) J. Heinzer, J. F. M. Oth, D. Seebach, Helv. Chim. Acta 1985, 68, 1848-1862; d) R. D. Thomas, M. T. Clarke, R. M. Jensen, T. C. Young, Organometallics 1986, 5, 1851-1857.

[40] A. J. Jones, D. M. Grant, J. G. Russell, G. Fraenkel, J. Phys. Chem. 1969, 73, 1624-1626.

[41] L. M. Jackman, L. M. Scarmoutzos, J. Am. Chem. Soc. 1984, 106, 4627-4629.

[42] a) H. Hope, P. P. Power, J. Am. Chem. Soc. 1983, 105, 5320-5324; b) D. Thoennes, E. Weiss, Chem. Ber. 1978, 111, 3157-3161; c) U. Schümann, J. Kopf, E. Weiss, Angew. Chem. 1985, 97, 222; Angew. Chem. Int. Ed. Engl. 1985, 24, 215-216.

[43] H. J. Reich, D. P. Green, M. A. Medina, W. S. Goldenberg, B. Ö. Gudmundsson, R. R. Dykstra, N. H. Philips, J. Am. Chem. Soc. 1998, 120, 7201-7210.

[44] J. F. McGarrity, C. A. Ogle, J. Am. Chem. Soc. 1985, 107, 1805-1810.

[45] a) J. F. McGarrity, J. Prodolliet, T. Smyth, Org. Magn. Reson. 1981, 17, 59-65; b) J. F. McGarrity, C. A. Ogle, Z. Brich, H. R. Loosli, J. Am. Chem. Soc. 1985, 107, 1810-1815.

[46] a) S. Macura, R. R. Ernst, Mol. Phys. 1980, 41, 95-117; b) S. Macura, Y. Huang, D. Suter, R. R. Ernst, J. Magn. Reson. 1981, 43, 259-281; c) S. Macura, K. Wuthrich, R. R. Ernst, J. Magn. Reson. 1982, 46, 269282.

[47] C. Yu, G. C. Levy, J. Am. Chem. Soc. 1983, 105, 6994-6996.

[48] P. L. Rinaldi, J. Am. Chem. Soc. 1983, 105, 5167-5168.

[49] C. Yu, G. C. Levy, J. Am. Chem. Soc. 1984, 106, 6533-6537.

[50] W. Bauer, G. Müller, R. Pi, P. von Ragué Schleyer, Angew. Chem. 1986, 98, 1130-1132; Angew. Chem. Int. Ed. Engl. 1986, 25, 1103-1104.

[51] W. Bauer, in Lithium Chemistry: A Theoretical and Experimental Overview (Eds.: A.-M. Sapse, P. v. R. Schleyer), John Wiley \& Sons, New York, 1995, pp. 125-172.

[52] R. M. Gschwind, P. R. Rajamohanan, M. John, G. Boche, Organometallics 2000, 19, 2868-2873.

[53] T. S. Claridge, in Tetrahedron Organic Chemistry Series, Vol. 27, 2 ed. (Eds.: J.-E. Bäckvall, J. E. Baldwin, R. M. Williams), Elsevier, Oxford, 2009, pp. 1-377.

[54] K. F. Morris, C. S. Johnson, Jr., J. Am. Chem. Soc. 1992, 114, 3141-3142.

[55] I. Keresztes, P. G. Williard, J. Am. Chem. Soc. 2000, 122, 10228-10229.

[56] E. J. Cabrita, S. Berger, Magn. Res. Chem. 2001, 39, S142-S148.

[57] D. Johnels, H. Günther, in PATAI'S Chemistry of Functional Groups, John Wiley \& Sons, Ltd, 2009. 
[58] M. Williamson, in Modern Magnetic Resonance (Ed.: G. Webb), Springer Netherlands, 2006, pp. 409412.

[59] a) M. Karplus, J. Am. Chem. Soc. 1963, 85, 2870-2871; b) M. J. Minch, Concepts Magn. Reson. 1994, 6, 41-56.

[60] R. R. Gil, Angew. Chem. 2011, 123, 7360-7362; Angew. Chem. Int. Ed. 2011, 50, 7222-7224.

[61] A. Saupe, Angew. Chem. 1968, 80, 99-115; Angew. Chem. Int. Ed. Engl. 1968, 7, 97-112.

[62] N. Tjandra, A. Bax, Science 1997, 278, 1111-1114.

[63] a) C. M. Thiele, Concepts Magn. Reson., Part A 2007, 30A, 65-80; b) C. M. Thiele, Eur. J. Org. Chem. 2008, 5673-5685.

[64] J. Yan, E. R. Zartler, Magn. Res. Chem. 2005, 43, 53-64.

[65] a) J. A. B. Lohman, C. MacLean, Mol. Phys. 1979, 38, 1255-1261; b) P. C. M. Van Zijl, B. H. Ruessink, J. Bulthuis, C. MacLean, Acc. Chem. Res. 1984, 17, 172-180.

[66] N. Tjandra, S. Grzesiek, A. Bax, J. Am. Chem. Soc. 1996, 118, 6264-6272.

[67] J. R. Tolman, J. M. Flanagan, M. A. Kennedy, J. H. Prestegard, PNAS 1995, 92, 9279-9283.

[68] M. John, A. Y. Park, G. Pintacuda, N. E. Dixon, G. Otting, J. Am. Chem. Soc. 2005, 127, 17190-17191.

[69] a) P. Haberz, F. Rodriguez-Castañeda, J. Junker, S. Becker, A. Leonov, C. Griesinger, Org. Lett. 2006, 8, 1275-1278; b) J. Wöhnert, K. J. Franz, M. Nitz, B. Imperiali, H. Schwalbe, J. Am. Chem. Soc. 2003, 125, 13338-13339.

[70] I. Bertini, C. Luchinat, G. Parigi, Concepts Magn. Reson. 2002, 14, 259-286.

[71] I. Bertini, A. Donaire, B. Jiménez, C. Luchinat, G. Parigi, M. Piccioli, L. Poggi, J Biomol NMR 2001, 21, 85-98.

[72] C. R. Sanders, J. P. Schwonek, Biochemistry 1992, 31, 8898-8905.

[73] C. R. Sanders, R. S. Prosser, Structure 1998, 6, 1227-1234.

[74] J. H. Prestegard, A. I. Kishore, Current Opin. Chem. Biol. 2001, 5, 584-590.

[75] T. B. Cardon, E. K. Tiburu, G. A. Lorigan, J. Magn. Reson. 2003, 161, 77-90.

[76] a) M. R. Hansen, L. Mueller, A. Pardi, Nat Struct Mol Biol 1998, 5, 1065-1074; b) J.-F. Trempe, F. Morin, Z. Xia, R. Marchessault, K. Gehring, J Biomol NMR 2002, 22, 83-87; c) M. Zweckstetter, G. Hummer, A. Bax, Biophys. J. 2004, 86, 3444-3460.

[77] Corrections for Zeri et al. PNAS 2007, 104, 2024.

[78] J. Baudry, E. Tajkhorshid, F. Molnar, J. Phillips, K. Schulten, J. Phys. Chem. B 2001, 105, 905-918.

[79] J. Sass, F. Cordier, A. Hoffmann, M. Rogowski, A. Cousin, J. G. Omichinski, H. Löwen, S. Grzesiek, J. Am. Chem. Soc. 1999, 121, 2047-2055. 
[80] v. h. c. o. This work is licensed under the Creative Commons Attribution-NonCommercial-ShareAlike 2.0 UK: England \& Wales License. To view a copy of this license.

[81] J. Courtieu, J. P. Bayle, B. M. Fung, Prog. Nucl. Magn. Reson. Spectrosc. 1994, 26, Part 2, 141-169.

[82] N. Suryaprakash, Concepts Magn. Reson. 1998, 10, 167-192.

[83] a) M. Rückert, G. Otting, J. Am. Chem. Soc. 2000, 122, 7793-7797; b) V. V. Klochkov, A. V. Klochkov, C. M. Thiele, S. Berger, J. Magn. Reson. 2006, 179, 58-63.

[84] a) M. Sarfati, P. Lesot, D. Merlet, J. Courtieu, Chem. Comm. 2000, 0, 2069-2081; b) C. Aroulanda, M. Sarfati, J. Courtieu, P. Lesot, Enantiomer 2001, 6, 281-287.

[85] T. J. Wenzel, Top. Curr. Chem. 2013, DOI 10.1007/128_2013_433.

[86] M. Dama, S. Berger, Tetrahedron Lett. 2012, 53, 6439-6442.

[87] a) A. Marx, C. Thiele, Chem. Eur. J. 2009, 15, 254-260; b) A. Marx, B. Böttcher, C. M. Thiele, Chem. Eur. J. 2010, 16, 1656-1663.

[88] a) B. Deloche, E. T. Samulski, Macromolecules 1981, 14, 575-581; b) Y. Rabin, E. T. Samulski, Macromolecules 1992, 25, 2985-2987.

[89] a) J. C. Freudenberger, P. Spiteller, R. Bauer, H. Kessler, B. Luy, J. Am. Chem. Soc. 2004, 126, 1469014691; b) B. Luy, K. Kobzar, H. Kessler, Angew. Chem. 2004, 116, 1112-1115; Angew. Chem. Int. Ed. 2004, 43, 1092-1094; c) J. C. Freudenberger, S. Knör, K. Kobzar, D. Heckmann, T. Paululat, H. Kessler, B. Luy, Angew. Chem. 2005, 117, 427-430; Angew. Chem. Int. Ed. 2005, 44, 423-426; d) B. Luy, K. Kobzar, S. Knör, J. Furrer, D. Heckmann, H. Kessler, J. Am. Chem. Soc. 2005, 127, 6459-6465; e) G. Kummerlöwe, J. Auernheimer, A. Lendlein, B. Luy, J. Am. Chem. Soc. 2007, 129, 6080-6081.

[90] a) G. Kummerlowe, S. Knor, A. O. Frank, T. Paululat, H. Kessler, B. Luy, Chem. Comm. 2008, 0, 57225724; b) G. Kummerlowe, M. Behl, A. Lendlein, B. Luy, Chem. Comm. 2010, 46, 8273-8275.

[91] R. R. Gil, C. Gayathri, N. V. Tsarevsky, K. Matyjaszewski, J. Org. Chem. 2008, 73, 840-848.

[92] P. Haberz, J. Farjon, C. Griesinger, Angew. Chem. 2005, 117, 431-433; Angew. Chem. Int. Ed. 2005, 44, 427-429.

[93] a) S. Meier, D. Häussinger, S. Grzesiek, J Biomol NMR 2002, 24, 351-356; b) T. Cierpicki, J. H. Bushweller, J. Am. Chem. Soc. 2004, 126, 16259-16266.

[94] a) H.-J. Sass, G. Musco, S. Stahl, P. Wingfield, S. Grzesiek, J Biomol NMR 2000, 18, 303-309; b) R. Tycko, F. J. Blanco, Y. Ishii, J. Am. Chem. Soc. 2000, 122, 9340-9341; c) J. Chou, S. Gaemers, B. Howder, J. Louis, A. Bax, J Biomol NMR 2001, 21, 377-382; d) G. Kummerlöwe, E. F. McCord, S. F. Cheatham, S. Niss, R. W. Schnell, B. Luy, Chem. Eur. J. 2010, 16, 7087-7089.

[95] F. Kramer, M. V. Deshmukh, H. Kessler, S. J. Glaser, Concepts Magn. Reson., Part A 2004, 21A, 10-21.

[96] J. Yan, A. D. Kline, H. Mo, M. J. Shapiro, E. R. Zartler, J Org Chem 2003, 68, 1786-1795.

[97] B. Böttcher, V. Schmidts, J. A. Raskatov, C. M. Thiele, Angew. Chem. 2010, 122, 210-214; Angew. Chem. Int. Ed. 2010, 49, 205-209. 
[98] a) L. Verdier, P. Sakhaii, M. Zweckstetter, C. Griesinger, J. Magn. Reson. 2003, 163, 353-359; b) C. Gayathri, M. C. de la Fuente, B. Luy, R. R. Gil, A. Navarro-Vazquez, Chem. Comm. 2010, 46, 5879-5881; c) G. Kummerlöwe, B. Crone, M. Kretschmer, S. F. Kirsch, B. Luy, Angew. Chem. 2011, 123, 2693-2696; Angew. Chem. Int. Ed. 2011, 50, 2643-2645; d) P. Tzvetkova, B. Luy, S. Simova, Magn. Res. Chem. 2012, 50, S92-S101.

[99] P. P. Man, in eMagRes, John Wiley \& Sons, Ltd, 2007.

[100]D. J. Philp, P. W. Kuchel, Concepts Magn. Reson., Part A 2005, 25A, 40-52.

[101]S. E. Ashbrook, S. Wimperis, in eMagRes, John Wiley \& Sons, Ltd, 2007.

[102]T. M. Clarke, P. J. Grandinetti, Journal of Physics: Condensed Matter 2003, 15, 2387-2395.

[103]A. D. Bain, M. Khasawneh, Concepts Magn. Reson., Part A 2004, 22A, 69-78.

[104]P. W. Kuchel, B. E. Chapman, N. Müller, W. A. Bubb, D. J. Philp, A. M. Torres, J. Magn. Reson. 2006, $180,256-265$

[105]D. J. Philp, C. Naumann, P. W. Kuchel, Concepts Magn. Reson., Part A 2012, 40A, 90-99.

[106]P. Crewdson, D. L. Bryce, F. Rominger, P. Hofmann, Angew. Chem. 2008, 120, 3503-3507; Angew. Chem. Int. Ed. 2008, 47, 3454-3457.

[107]A. S. Lipton, R. W. Heck, W. A. de Jong, A. R. Gao, X. Wu, A. Roehrich, G. S. Harbison, P. D. Ellis, J. Am. Chem. Soc. 2009, 131, 13992-13999.

[108]Z. Yu, A. Zheng, Q. Wang, L. Chen, J. Xu, J.-P. Amoureux, F. Deng, Angew. Chem. 2010, 122, 88398843; Angew. Chem. Int. Ed. 2010, 49, 8657-8661.

[109]Y. Wei, R. Graf, J. C. Sworen, C.-Y. Cheng, C. R. Bowers, K. B. Wagener, H. W. Spiess, Angew. Chem. 2009, 121, 4687-4690; Angew. Chem. Int. Ed. 2009, 48, 4617-4620.

[110] L. Holmes, L. Peng, I. Heinmaa, L. A. O’Dell, M. E. Smith, R.-N. Vannier, C. P. Grey, Chem. Mater. 2008, 20, 3638-3648.

[111]F. Hallwass, M. Schmidt, H. Sun, A. Mazur, G. Kummerlöwe, B. Luy, A. Navarro-Vázquez, C. Griesinger, U. M. Reinscheid, Angew. Chem. 2011, 123, 9659-9662; Angew. Chem. Int. Ed. 2011, 50, 9487-9490.

[112]G. Cornilescu, J. L. Marquardt, M. Ottiger, A. Bax, J. Am. Chem. Soc. 1998, 120, 6836-6837.

[113]a) J. W. Emsley, J. C. Lindon, NMR spectroscopy using liquid crystal solvents, Pergamon Press, 1975; b) P. Tzvetkova, B. Luy, S. Simova, in Topics in Chemistry and Material Science, Vol. 5 (Eds.: R. D. Nikolova, S. Simova, P. Denkova, G. N. Vayssilov), 2011, pp. 70-77.

[114]A. Grishaev, L. Yao, J. Ying, A. Pardi, A. Bax, J. Am. Chem. Soc. 2009, 131, 9490-9491.

[115]a) D. Stalke, Angew. Chem. 1994, 106, 2256-2259; Angew. Chem. Int. Ed. 1994, 33, 2168-2171; b) W. N. Setzer, P. v. R. Schleyer, Adv. Organomet. Chem. 1985, 24, 353-451; c) P. v. R. Schleyer, Pure Appl. Chem. 1983, 55, 355-362.

[116]a) D. B. Collum, Acc. Chem. Res. 1992, 25, 448-454; b) K. L. Jantzi, C. L. Puckett, I. A. Guzei, H. J. Reich, J. Org. Chem. 2005, 70, 7520-7529. 
[117] a) H. W. Gschwend, H. R. Rodriguez, Org. React. 1979, 26, 1; b) V. Snieckus, Chem. Rev. 1990, 90, 879933; c) M. Stratakis, J. Org. Chem. 1997, 62, 3024-3025; d) D. W. Slocum, S. Dubris, S. Brown, G. Jackson, R. LaMastus, E. Mullins, J. Ray, P. Shelton, A. Walstrom, J. M. Wilcox, R. W. Holman, Tetrahedron 2003, 59, 8275-8284.

[118]a) A. R. Lepley, W. A. Khan, A. B. Giumanini, A. G. Giumanini, J. Org. Chem. 1966, 31, 2047-2051; b) S.-I. Murahashi, T. Naota, Y. Tanigawa, Organic Syntheses; Wiley: New York, 1984; 1984, Vol. 62, 39; c) M. Cyranski, T. M. Krygowski, A. R. Katritzky, P. v. R. Schleyer, J. Org. Chem. 2002, 67, 1333-1338.

[119]K. Chernichenko, M. Nieger, M. Leskelä, T. Repo, Dalton Trans. 2012, 41, 9029-9032.

[120]a) W. Bauer, P. v. R. Schleyer, J. Am. Chem. Soc. 1989, 111, 7191-7198; b) N. J. R. van Eikema Hommes, P. v. R. Schleyer, Tetrahedron 1994, 50, 5903-5916.

[121]M. M. Meinholz, PhD Thesis, Göttingen (Germany) 2011.

[122]J. T. B. H. Jastrzebski, G. van Koten, M. Konijn, C. H. Stam, J. Am. Chem. Soc. 1982, 104, 5490-5492.

[123]D. Bojer, I. Kamps, X. Tian, A. Hepp, T. Pape, R. Fröhlich, N. W. Mitzel, Angew. Chem. 2007, 119, 4254-4257; Angew. Chem. Int. Ed. 2007, 46, 4176-4179.

[124]M. C. Etter, Z. Urbanczyk-Lipkowska, M. Zia-Ebrahimi, T. W. Panunto, J. Am. Chem. Soc. 1990, 112, 8415-8426.

[125]a) J. Urbanus, C. P. M. Roelands, D. Verdoes, P. J. Jansens, J. H. ter Horst, Cryst. Growth Des. 2010, 10, 1171-1179; b) G. P. Vitorino, N. R. Sperandeo, M. R. Caira, M. R. Mazzieri, Cryst. Growth Des. 2013, dx.doi.org/10.1021/cg301299e.

[126]S. Harder, J. Boersma, L. Brandsma, G. P. M. van Mier, J. A. Kanters, J. Organomet. Chem. 1989, 364, 115.

[127]S. Harder, M. Lutz, T. Kremer, Organometallics 1995, 14, 2133-2135.

[128]S. Zhang, Å. C. Rasmuson, Cryst. Growth Des. 2013, 13, 1153-1161.

[129]P. Wijkens, E. M. van Koten, M. D. Janssen, J. T. B. H. Jastrzebski, A. L. Spek, G. van Koten, Angew. Chem. 1995, 107, 239-242; Angew. Chem. Int. Ed. 1995, 34, 219-222.

[130]a) R. D. Thomas, H. Huang, J. Am. Chem. Soc. 1999, 121, 11239; b) X. Sun, M. D. Winemiller, B. Xiang, D. B. Collum, J. Am. Chem. Soc. 2001, 123, 8039-8046.

[131]a) G. Fraenkel, A. M. Fraenkel, M. J. Geckle, F. Schloss, J. Am. Chem. Soc. 1979, 101, 4745-4747; b) G. Fraenkel, M. Henrichs, J. M. Hewitt, B. M. Su, M. J. Geckle, J. Am. Chem. Soc. 1980, 102, 3345-3350.

[132]a) G. van Koten, J. G. Noltes, J. Organomet. Chem. 1975, 84, 129; b) G. van Koten, J. G. Noltes, J. Organomet. Chem. 1979, 174, 367-387.

[133]a) H. J. Reich, W. S. Goldenberg, B. Ö. Gudmundsson, A. W. Sanders, K. J. Kulicke, K. Simon, I. A. Guzei, J. Am. Chem. Soc. 2001, 123, 8067-8079; b) H. J. Reich, W. S. Goldenberg, A. W. Sanders, K. L. Jantzi, C. C. Tzschucke, J. Am. Chem. Soc. 2003, 125, 3509-3521; c) H. J. Reich, W. S. Goldenberg, A. W. Sanders, ARKIVOC 2004, xiii, 97-129.

[134]a) F. Petti, A. Thelemann, J. Kahler, S. McCormack, L. Castaldo, T. Hunt, L. Nuwaysir, L. Zeiske, H. Haack, L. Sullivan, A. Garton, J. D. Haley, Mol Cancer Ther 2005, 4, 1186-1197; b) N. Sweeny, W. M. 
Gallagher, H. Müller-Bunz, C. Pampillón, K. Strohfeldt, M. Tacke, J. Inorg. Biochem. 2006, 100, 14791486.

[135]G. B. Bachman, L. V. Heisey, J. Am. Chem. Soc. 1948, 70, 2378-2380.

[136]T. Izumi, S. Kobashi, K. Takimiya, Y. Aso, T. Otsubo, J. Am. Chem. Soc. 2003, 125, 5286-5287.

[137]Y. Liu, Y. Liu, X. Zhan, Macromol. Chem. Phys. 2011, 212, 428-443.

[138]D. Belo, M. Almeida, Coord. Chem. Rev. 2010, 254, 1479-1492.

[139]a) R. Chinchilla, C. Nájera, M. Yus, Chem. Rev. 2004, 104, 2667-2722; b) R. Chinchilla, C. Nájera, M. Yus, ARKIVOC 2007, 10, 152-231.

[140]C. Nájera, J. M. Sansano, M. Yus, Tetrahedron 2003, 59, 9255-9303.

[141]H. Gilman, D. A. Shirley, J. Am. Chem. Soc. 1949, 71, 1870-1871.

[142]a) K. Shen, Y. Fu, J.-N. Li, L. Liu, Q.-X. Guo, Tetrahedron 207, 63, 1568-1576; b) R. R. Fraser, T. S. Mansour, S. Savard, Can. J. Chem. 1985, 63, 3505-3509.

[143]C. Peyron, J.-M. Navarre, N. Van Craynest, R. Benhida, Tetrahedron Lett. 2005, 46, 3315-3318.

[144]P. Wagner, A. M. Ballantyne, K. W. Jolley, D. L. Officer, Tetrahedron 2006, 62, 2190-2199.

[145] in Cambridge Structural Database, v5.34 (November 2012), Cambridge Crystallographic Data Centre, Cambridge, 2012.

[146]A. L. Spek, W. J. J. Smeets, 1999, Private Communication to the CCDC (113718).

[147]S. Gronowitz, B. Andreas, Acta Chem. Scand. 1968, 22, 59-62.

[148]A. Dabrowski, K. Kamienska-Trela, J. Wojcik, Spectrochim. Acta, Part A 1999, 56, 91-102.

[149]W. Bauer, C. Griesinger, J. Am. Chem. Soc. 1993, 115, 10871.

[150]a) P. Lazzeretti, L. Schenetti, F. Taddei, P. Spagnolo, M. Tiecco, Org. Magn. Reson. 1972, 4, 413-419; b) B. Abarca, G. Sensio, R. Ballestros, T. Varea, J. Org. Chem. 1991, 56, 3224-3229.

[151]M. Granitzka, A.-C. Pöppler, E. K. Schwarze, D. Stern, T. Schulz, M. John, R. Herbst-Irmer, S. K. Pandey, D. Stalke J. Am. Chem. Soc. 2012, 134, 1344-1351.

[152]M. Karplus, J. A. Pople, J. Chem. Phys. 1963, 38, 2803-2807.

[153]W. Bauer, W. R. Winchester, P. v. R. Schleyer, Organometallics 1987, 6, 2371-2379.

[154]C. G. Screttas, J. F. Eastham, J. Am. Chem. Soc. 1965, 87, 3276-3277.

[155]D. Neuhaus, M. P. Williamson, The Nuclear Overhauser Effect in Structural and Confomational Analysis, VCH: New York, 1989.

[156]A. M. Gronenborn, G. M. Clore, Prog. Nucl. Magn. Reson. Spectrosc. 1985, 17, 1-36.

[157]K. S. Khuong, W. H. Jones, W. A. Pryor, K. N. Houk, J. Am. Chem. Soc. 2005, 127, 1265-1277. 
[158]A. Boman, D. Johnels, Magn. Res. Chem. 2000, 38, 853-860.

[159]C. Strohmann, K. Strohfeldt, D. Schildbach, J. Am. Chem. Soc. 2003, 125, 13672-13673.

[160]A. Navarro-Vázquez, Magn. Res. Chem. 2012, 50, S73-S79.

[161]a) U. Wannagat, H. Niederprüm, Chem. Ber. 1961, 94, 1540-1547; b) D. B. Collum, Acc. Chem. Res. 1993, 26, 227-234.

[162]D. E. Woessner, Concepts Magn. Reson. 2001, 13, 294-325.

[163]a) B. L. Lucht, D. B. Collum, J. Am. Chem. Soc. 1994, 116, 6009-6010; b) S. Popenova, R. c. Mawhinney, G. Schreckenbach, Inorg. Chem. Commun. 2007, 46, 3856-3864.

[164]P. Stanetty, M. D. Mihovilovic, J. Org. Chem. 1996, 62, 1514-1515.

[165]C. Strohmann, V. H. Gessner, Angew. Chem. 2007, 119, 4650-4653; Angew. Chem. Int. Ed. 2007, 46, 4566-4569.

[166]N. D. R. Barnett, R. E. Mulvey, W. Clegg, P. A. O'Neil, J. Am. Chem. Soc. 1993, 115, 1573-1574.

[167]a) J. G. Snijders, C. A. de Lange, E. E. Burnell, J. Chem. Phys. 1983, 79, 2964-2969; b) C. A. de Lange, W. L. Meerts, A. C. J. Weber, E. E. Burnell, J. Phys. Chem. A 2010, 114, 5878-5887.

[168]D. Johnels, H. Günther, in The chemistry of organolithium compounds (Eds.: Z. Rappoport, I. Marek), John Wiley \& Sons, Chichester (GB), 2004, pp. 137-203.

[169]L. M. Jackman, L. M. Scarmoutzos, C. W. DeBrosse, J. Am. Chem. Soc. 1986, 109, 5355-5361.

[170]J. W. Zwanziger, M. Torrent, Appl. Magn. Reson. 2008, 33, 447-456.

[171]a) M. A. Bernstein, K. F. King, X. J. Zhou, Handbook of MRI Pulse Sequences, Elsevier Science, 2004; b) D. Weishaupt, V. D. Köchli, B. Marincek, K. P. Pruessmann, J. M. Froehlich, How Does MRI Work?: An Introduction to the Physics and Function of Magnetic Resonance Imaging, Springer-Verlag Berlin Heidelberg, 2006; c) M. A. Brown, R. C. Semelka, MRI: Basic Principles and Applications, Wiley, 2011.

[172]a) A. N. Garroway, P. K. Grannell, P. Mansfield, J. Phys. C: Solid State Phys. 1974, 7, L457; b) P. C. Lauterbur, D. M. Kramer, W. V. House, C.-N. Chen, J. Am. Chem. Soc. 1975, 97, 6866-6868.

[173]a) C. Bauer, R. Freeman, T. Frenkiel, J. Keeler, A. J. Shaka, J. Magn. Reson. 1984, 58, 442-457; b) L. Emsley, G. Bodenhausen, Chem. Phys. Lett. 1990, 165, 469-476.

[174]D. Kim, J. M. Caruthers, N. A. Peppas, Macromolecules 1993, 26, 1841-1847.

[175]a) M. Ilg, B. Pfleiderer, K. Albert, W. Rapp, E. Bayer, Macromolecules 1994, 27, 2778-2783; b) P. J. McDonald, J. Godward, R. Sackin, R. P. Sear, Macromolecules 2001, 34, 1048-1057; c) F. Tabak, M. Corti, J. Chem. Phys. 1990, 92, 2673; d) R. Sackin, E. Ciampi, J. Godward, J. L. Keddie, P. J. McDonald, Macromolecules 2001, 34, 890-895.

[176]J. E. Mark, Polymer data handbook, Oxford University Press, Incorporated, 1999.

[177] M. C. Grady, W. J. Simonsick, R. A. Hutchinson, Macromol. Symp. 2002, 182, 149-168. 
[178]a) E. F. McCord, W. H. Shaw, R. A. Hutchinson, Macromolecules 1997, 30, 246-256; b) A. N. F. Peck, R. A. Hutchinson, Macromolecules 2004, 37, 5944-5951.

[179]a) C. Plessis, G. Arzamendi, J. R. Leiza, H. A. S. Schoonbrood, D. Charmot, J. M. Asua, Macromolecules 2000, 33, 5041-5047; b) C. Plessis, G. Arzamendi, J. M. Alberdi, A. M. van Herk, J. R. Leiza, J. M. Asua, Macromol. Rapid Commun. 2003, 24, 173-177; c) J. M. Asua, S. Beuermann, M. Buback, P. Castignolles, B. Charleux, R. G. Gilbert, R. A. Hutchinson, J. R. Leiza, A. N. Nikitin, J.-P. Vairon, A. M. van Herk, Macromol. Chem. Phys. 2004, 205, 2151-2160.

[180]a) A. N. Nikitin, R. A. Hutchinson, M. Buback, P. Hesse, Macromolecules 2007, 40, 8631-8641; b) J. Barth, M. Buback, P. Hesse, T. Sergeeva, Macromolecules 2010, 43, 4023-4031.

[181]J. Barth, M. Buback, P. Hesse, T. Sergeeva, Macromol. Rapid Commun. 2009, 30, 1969-1974.

[182]B. Liu, A. Kazlauciunas, J. T. Guthrie, S. Perrier, Macromolecules 2005, 38, 2131-2136.

[183]E. S. Matsuo, M. Orkisz, S.-T. Sun, Y. Li, T. Tanaka, Macromolecules 1994, 27, 6791-6796.

[184]K. Matyjaszewski, in Controlled/Living Radical Polymerization, Vol. 768, American Chemical Society, 2000, pp. 2-26.

[185]a) J.-S. Wang, K. Matyjaszewski, J. Am. Chem. Soc. 1995, 117, 5614-5615; b) M. Kato, M. Kamigaito, M. Sawamoto, T. Higashimura, Macromolecules 1995, 28, 1721-1723; c) D. M. Haddleton, M. C. Crossman, B. H. Dana, D. J. Duncalf, A. M. Heming, D. Kukulj, A. J. Shooter, Macromolecules 1999, 32, 2110-2119.

[186]a) J. Chiefari, Y. K. Chong, F. Ercole, J. Krstina, J. Jeffery, T. P. T. Le, R. T. A. Mayadunne, G. F. Meijs, C. L. Moad, G. Moad, E. Rizzardo, S. H. Thang, Macromolecules 1998, 31, 5559-5562; b) Y. K. Chong, T. P. T. Le, G. Moad, E. Rizzardo, S. H. Thang, Macromolecules 1999, 32, 2071-2074; c) G. Moad, J. Chiefari, Y. K. Chong, J. Krstina, R. T. A. Mayadunne, A. Postma, E. Rizzardo, S. H. Thang, Polym. Int. 2000, 49, 993-1001.

[187]S. Perrier, C. Barner-Kowollik, J. F. Quinn, P. Vana, T. P. Davis, Macromolecules 2002, 35, 8300-8306.

[188]N. Ide, T. Fukuda, Macromolecules 1998, 32, 95-99.

[189]T. Norisuye, T. Morinaga, Q. Tran-Cong-Miyata, A. Goto, T. Fukuda, M. Shibayama, Polymer 2005, 46, 1982-1994.

[190]Q. Lio, P. Zhang, A. Qing, Y. Lan, M. Lu, Polymer 2006, 47, 2330-2336.

[191]Q. Yu, Y. Zhu, Y. Ding, S. Zhu, Macromol. Chem. Phys. 2008, 209, 551-556.

[192]D. W. Stephan, G. Erker, Angew. Chem. 2010, 122, 50-81; Angew. Chem. Int. Ed. 2010, 49, 46-76.

[193]V. Grignard, C. R. Hebd. Seances Acad. Sci 1900, 130, 1322-1324.

[194]H. G. Richey, Grignard reagents: new developments, Wiley, 2000.

[195]C. Elschenbroich, in Organometallchemie - Eine kurze Einführung, Vol. 6, Teubner, Stuttgart, 2008.

[196]R. M. Salinger, H. S. Mosher, J. Am. Chem. Soc. 1964, 86, 1782-1786.

[197]E. C. Ashby, G. E. Parris, J. Am. Chem. Soc. 1971, 93, 1206-1213. 
[198]S. Sakamoto, T. Imamoto, K. Yamaguchi, Org. Lett. 2001, 3, 1793-1795.

[199] A. Krasovskiy, P. Knochel, Angew. Chem. 2004, 116, 3396-3399; Angew. Chem. Int. Ed. 2004, 43, 33333336.

[200] A. Krasovskiy, B. F. Straub, P. Knochel, Angew. Chem. 2006, 118, 165-169; Angew. Chem. Int. Ed. 2006, $45,159-162$.

[201]F. Blasberg, M. Bolte, M. Wagner, H.-W. Lerner, Organometallics 2012, 31, 1001-1005.

[202]P. García-Álvarez, D. V. Graham, E. Hevia, A. R. Kennedy, J. Klett, R. E. Mulvey, C. T. O'Hara, S. Weatherstone, Angew. Chem. 2008, 120, 8199-8201; Angew. Chem. Int. Ed. 2008, 47, 8079-8081.

[203]a) E. C. Ashby, W. E. Becker, J. Am. Chem. Soc. 1963, 85, 118-119; b) J. Toney, G. D. Stucky, J. Organomet. Chem. 1971, 28, 5-20.

[204]a) S. H. Bauer, T. Ino, R. F. Porter, J. Chem. Phys. 1960, 33, 685-691; b) W. Ming Keong, A. I. Popov, J. Inorg. Nucl. Chem. 1972, 34, 3615-3622.

[205]a) R. E. Mulvey, Acc. Chem. Res. 2009, 42, 743-755; b) S. E. Baillie, W. Clegg, P. García-Álvarez, E. Hevia, A. R. Kennedy, J. Klett, L. Russo, Organometallics 2012, 31, 5131-5142.

[206]a) H. Gornitzka, D. Stalke, Angew. Chem. 1994, 106, 695-698; Angew. Chem. Int. Ed. 1994, 33, 693-695; b) F. Pauer, J. Rocha, D. Stalke, J. Chem. Soc., Chem. Commun. 1991, 20, 1477-1479; c) S. Freitag, W. Kolodziejski, F. Pauer, D. Stalke, J. Chem. Soc., Dalton Trans. 1993, 3479-3488.

[207]U. J. Bildmann, G. Müller, Organometallics 2001, 20, 1689-1691.

[208]a) A. D. Becke, Phys. Rev. A 1988, 38, 3098-3100; b) J. P. Perdew, Phys. Rev. B 1986, 33, 8822-8824; c) A. Schafer, H. Horn, R. Ahlrichs, J. Chem. Phys. 1992, 97, 2571-2577; d) S. Grimme, J. Comput. Chem. 2006, 27, 1787-1799; e) ORCA, v. 2.8, F. Neese, U. Becker, D. Ganiouchine, S. Koßmann, T. Petrenko, C. Riplinger, F. Wennmohs, University of Bonn (Germany), 2010; f) S. Grimme, J. Antony, S. Ehrlich, H. Krieg, J. Chem. Phys. 2010, 132, 154104-154119.

[209]T. Teuterberg, Bachelor Thesis, Göttingen (Germany) 2011.

[210]A. Klamt, G. Schuurmann, J. Chem. Soc., Perkin Trans. 2 1993, 799-805.

[211]F. Weigend, R. Ahlrichs, Phys. Chem. Chem. Phys. 2005, 7, 3297-3305.

[212]H.-J. Werner, M. Schutz, J. Chem. Phys. 2011, 135, 144116-144115.

[213]J. T. H. Dunning, J. Chem. Phys. 1989, 90, 1007-1023.

[214]B. Prascher, D. Woon, K. Peterson, T. Dunning, Jr., A. Wilson, Theor Chem Acc 2011, 128, 69-82.

[215]F. Weigend, Phys. Chem. Chem. Phys. 2002, 4, 4285-4291.

[216]F. Weigend, J. Comput. Chem. 2008, 29, 167-175.

[217]F. Weigend, A. Kohn, C. Hattig, J. Chem. Phys. 2002, 116, 3175-3183.

[218]J. G. Hill, K. A. Peterson, Phys. Chem. Chem. Phys. 2010, 12, 10460-10468. 
[219]W. Schlenk, in Die Methoden der Organischen Chemie, 2. Aufl. ed. (Ed.: J. Houben), G. Thieme, Leipzig, 1924, p. 720.

[220]W. G. Kofron, L. M. Baclawski, J. Org. Chem. 1976, 41, 1879-1880. 
